Portland State University

PDXScholar

$12-2-2020$

\title{
Aligning Food Environments With Institutional Values: A Mixed Methods Study of Oregon Health Care Organizations
}

Elizaveta Walker

Portland State University

Follow this and additional works at: https://pdxscholar.library.pdx.edu/open_access_etds

Part of the Food Science Commons, Health Policy Commons, and the Public Health Commons Let us know how access to this document benefits you.

Recommended Citation

Walker, Elizaveta, "Aligning Food Environments With Institutional Values: A Mixed Methods Study of Oregon Health Care Organizations" (2020). Dissertations and Theses. Paper 5621.

https://doi.org/10.15760/etd.7493

This Dissertation is brought to you for free and open access. It has been accepted for inclusion in Dissertations and Theses by an authorized administrator of PDXScholar. Please contact us if we can make this document more accessible: pdxscholar@pdx.edu. 
Aligning Food Environments with Institutional Values: A Mixed Methods Study of Oregon Health Care Organizations

by

Elizaveta Walker

A dissertation submitted in partial fulfillment of the requirements for the degree of

Doctor of Philosophy

in

Health Systems and Policy

Dissertation Committee:

Julia Marie Goodman, Chair

Robin Lynn Baker

Lynne Corinne Messer

Cara Louise Eckhardt

Kerth O'Brien

Portland State University

2020 
(C) 2020 Elizaveta Walker 


\begin{abstract}
A major driver of the obesity epidemic is obesogenic food environments, characterized by nutrient-poor and energy-dense foods that saturate the collective physical, economic and sociocultural conditions that influence nutritional status. Food environments in organizations such as hospitals and public health agencies warrant special consideration given their health-focused mission. Improving food environments within health care settings has been highlighted by the Centers for Disease Control and Prevention (CDC) as one of seven key strategies to prevent obesity. However, most of the refereed literature examining healthy food environment policies (HFEPs) within hospitals focuses on the inpatient dietary environment, leaving a paucity of information on facilitators of or barriers to implementation.
\end{abstract}

This dissertation study sought to examine the relationship between organizational characteristics and the selection, implementation, and outcomes of HFEPs within six health care organizations. The overarching research question was: How does the organizational context of health care organizations affect the implementation process and economic outcomes of healthy food environment policies? Aim 1 sought to qualitatively describe the barriers to and facilitators of implementing HFEPs among two levels of leadership: operational managers and executive leaders. Semi-structured key informant interviews revealed 27 facilitators and 30 barriers cited among ten respondents. Examining leadership perceptions, operational and executive leaders overlapped $44-75 \%$ when identifying facilitators but only $33-58 \%$ when identifying 
barriers to HFEP implementation. Aim 2 sought to evaluate the revenue and consumer behavior outcomes of a sugar-sweetened beverage (SSB) ban established within a nonprofit regional health care system. Utilizing an interrupted time-series analysis, findings demonstrated no statistically significant decrease in gross monthly sales 6,12 , and 18 months after the ban. Increases in water, juice, coffee and sports drinks sales after the ban indicate that consumer purchasing shifted from SSBs toward healthier options. Despite the continued availability of diet SSBs offered post-ban, monthly SSB sales decreased by a mean of $44.7 \%$. Finally, Aim 3 sought to describe the relationship between organizational contexts and HFEP selection. Findings showcased the spectrum of HFEPs adopted and how organizational contexts presented distinct opportunities and challenges during the implementation process. For example, centralized governance models were effective for HFEP development when coupled with resource commitments but not effective when adverse stakeholders created roadblocks for HFEP opportunities. Sustainability commitments drove HFEP development when coupled with an executive champion; otherwise, such commitments led to staff apathy. Contextual recommendations synthesized the experiences of each organization, noting similarities and differences.

Examined together, these three papers provide meaningful theoretical and practical insights into the selection, implementation, and outcomes of HFEP development. Earlier chapters initially discussed how the selection and implementation of HFEPs have historically underestimated the importance of organizational theory, with implementation toolkits and step-by-step guides often mentioning the need for a 
"champion" or "resources" but saying little else about the role of the organizational setting in determining what kinds of HFEPs would be better suited in which settings. Aims 1 and 3 introduced a rich examination of both the organizational contexts of six diverse health care organizations as well as the facilitators and barriers cited during the HFEP implementation processes of these institutions. When complementing the qualitative inquiries with the quantitative findings of Aim 2, the findings provide evidence of the revenue and consumer behavior outcomes of a sugar-sweetened beverage ban, a HFEP that is growing in popularity and uptake. By blending these process-oriented and outcome-oriented queries, prospective decision-makers can feel equipped and wellinformed to proceed with HFEP selection, implementation, and evaluation. 


\section{DEDICATION}

To my mother, Natalie Alekseevna Kvach

It is thanks to your foundation of love that I grew up with a firm sense of belief in myself. It is thanks to your discipline and fairness that I forged a work ethic. It is thanks to your unfailing confidence in me that I decided to pursue a doctoral education. While I can never repay you for all of your sacrifices, I hope I can model the same love for my daughter and raise her to always set high standards, nurture a sense of self, and know she can achieve anything in this world. Спасибо, Мамочка.

To my husband, Daniel

This work could be as much yours as it is mine. You could describe to any stranger the nuances of my dissertation, and it's been a personal joy to have a partner who took such an interest in my little corner of the world.

All I can say is I love you, thank you, and I promise this is my last degree. 


\section{ACKNOWLEDGMENTS}

I will remember this journey not by the courses taken or exams completed but rather by the mentors and friends who provided guidance, camaraderie and lifelong friendships. I extend my gratitude foremost to my dissertation committee. To my chair, Julia Goodman—having a child during this program could have terminally derailed my progress if I had found myself in another program, another time, or under another chair. Instead, you offered advice and accommodation, and through that I felt I had permission to enjoy, not rue, the process of balancing motherhood and graduate work.

Sincere thanks go to Lynne Messer, Robin Baker, Cara Eckhardt and Kerth O'Brien. I am indebted to each of you and I am proud to have found myself among a circle of such formidable women. Through many hours of office meetings and reviews of drafts, I am lucky I get to count each of you as mentors and friends.

Perhaps my biggest debt and deepest thanks go to Sherril Gelmon. I began my academic journey nearly a decade ago when I walked into your master-level course to learn about health systems organization. Two programs later, I have relied on your mentorship and advice to navigate every major academic and professional decision. You model excellence in every way, setting high standards and never second-guessing my ability to reach them. You are changing the world one student at a time.

To my fellow graduate students who made coursework and seminars enjoyable: thank you. To my mother-in-law who watched my little girl so I could write: thank you. And to the rest of my family: thank you for your flexibility, grace, and support. 


\section{TABLE OF CONTENTS}

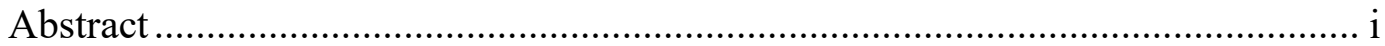

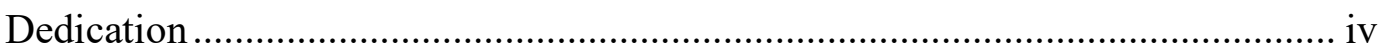

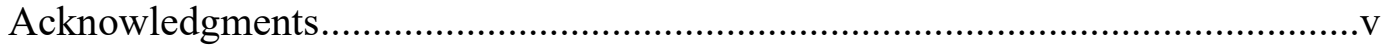

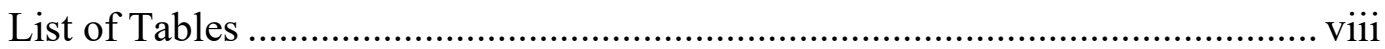

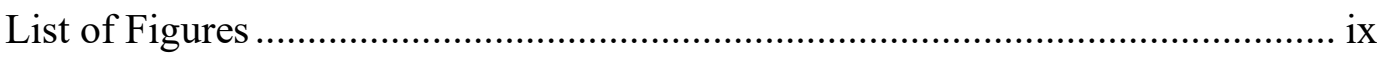

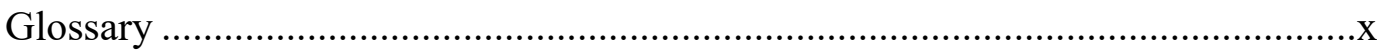

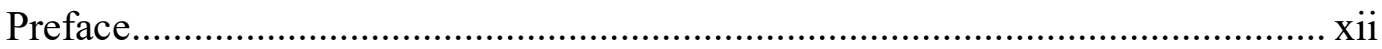

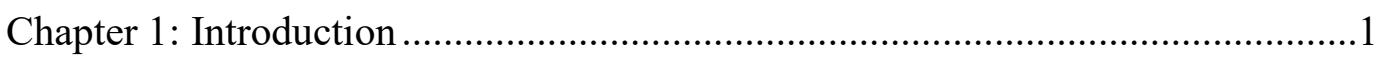

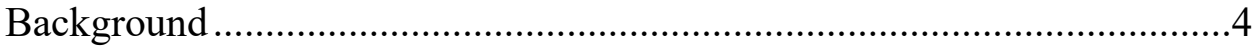

Statement of the Problem......................................................................13

Research Question .............................................................................

Theoretical Framework ........................................................................16

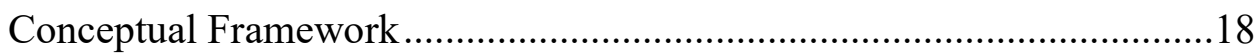

Purpose and Significance of the Study …………………......................20

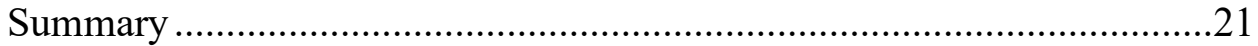

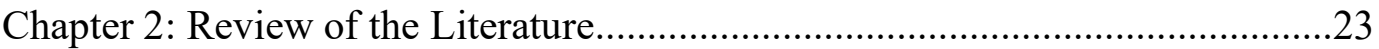

Obesity Epidemic: Framing, Drivers, and Prevention Strategies ...............23

Healthy Food Environment Policies ...........................................................41

Role of Health care Institutions in Addressing the Obesity Epidemic ......55

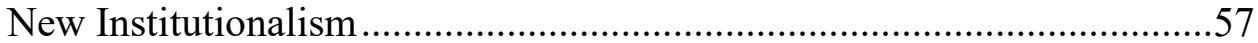

The Role of Organizational Context ……………………..........................59

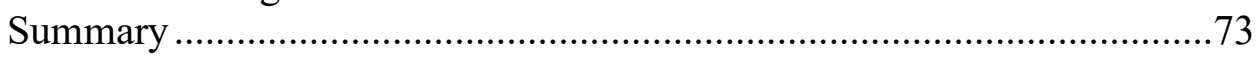

Chapter 3: Methodology ………………………………...................................76

Operationalization of Key Concepts .......................................................76

Overview of Research Design and Rationale ..........................................79

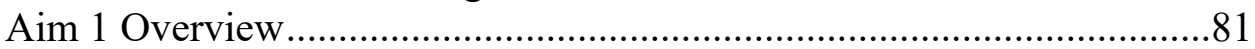

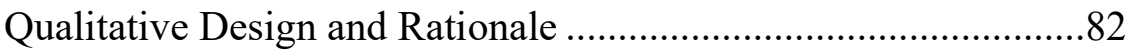

Selection of Participating Organizations ……………...................83

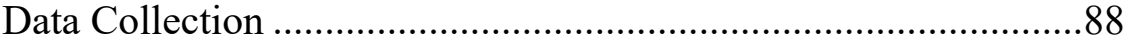

Interview Guide Development......................................................

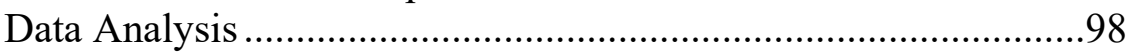

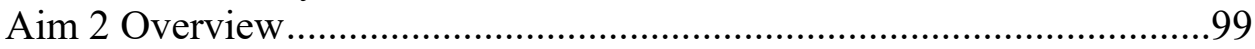

Quantitative Design and Rationale ............................................101

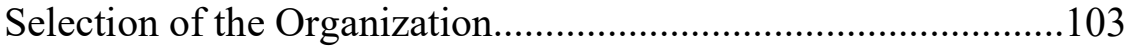

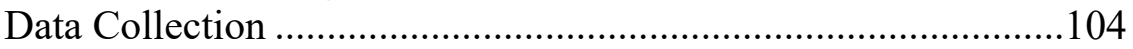

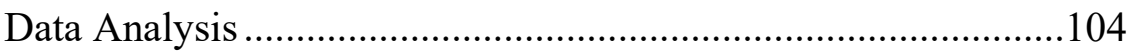

Aim 3 Overview 106 


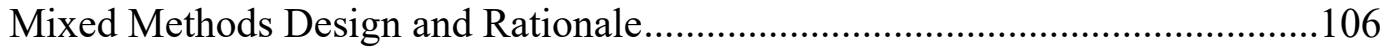

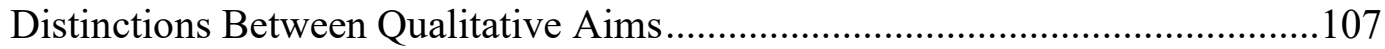

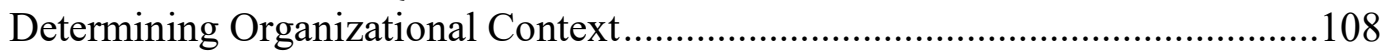

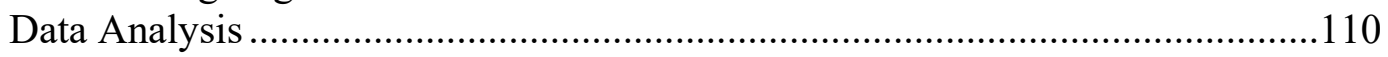

Chapter 4: Improving the Food Environment: Determining the

Role of Leadership Perceptions on Implementation Challenges ........................111

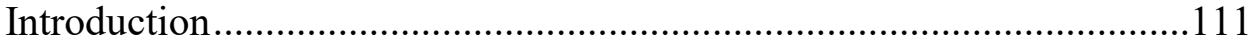

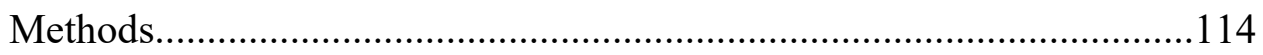

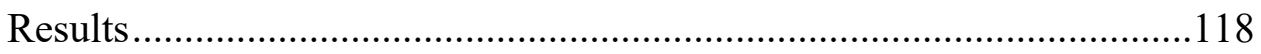

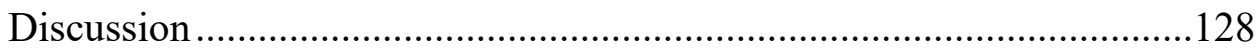

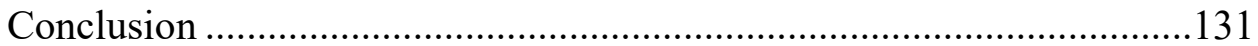

Chapter 5: An Interrupted Time-Series Analysis to Evaluate a

Health System's Sugar-Sweetened Beverage Sales Ban: Outcomes

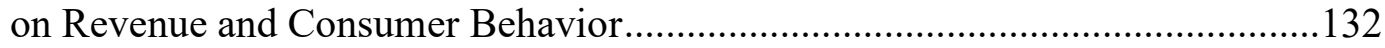

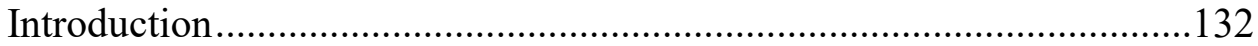

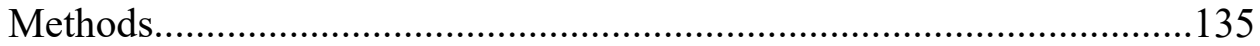

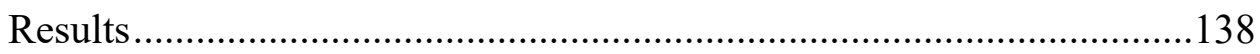

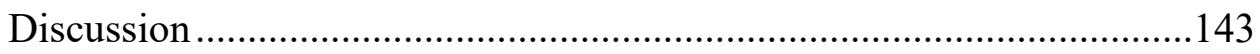

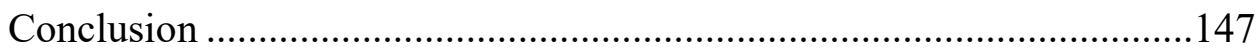

Chapter 6: The Role of Organizational Context in Shaping HFEP

Adoption and Implementation Challenges........................................................148

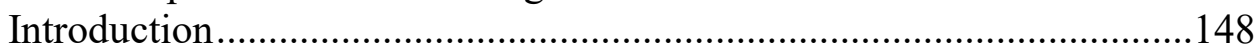

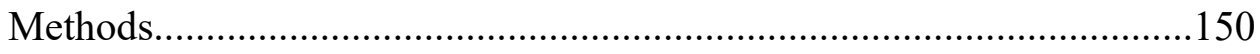

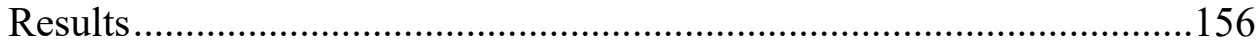

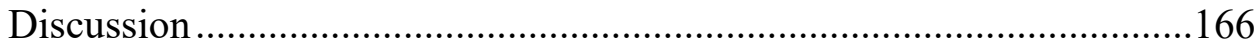

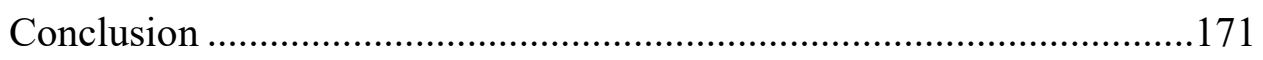

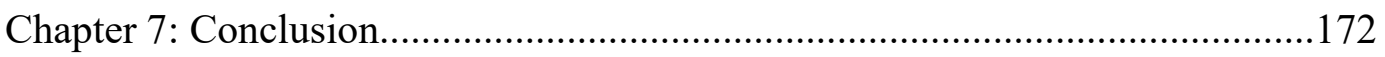

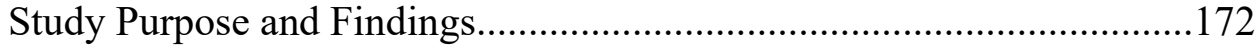

Synthesis of the Dissertation Papers ...................................................177

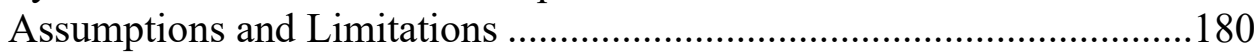

Dissemination Plan ............................................................... 183

Implications for Future Research ............................................... 184

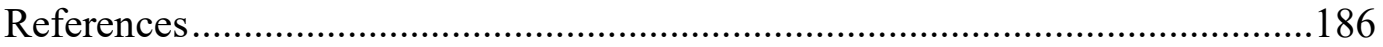

Appendix A: Interview Protocol .................................................................217

Appendix B: Recruitment Email Invitation to Interview Participants ................220

Appendix C: Institutional Review Board Consent Form .................................222

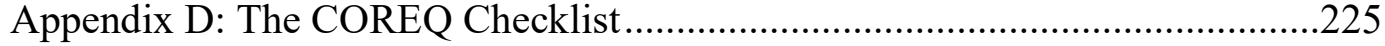

Appendix E: Organizational Context Scores for All Variables ..........................227 


\section{LIST OF TABLES}

Table 3.1 Operationalization of Key Concepts.......................................................77

Table 3.2 Operationalization of Organizational Context.........................................78

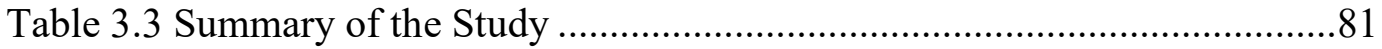

Table 3.4 Conceptual Domains and Interview Questions.......................................97

Table 3.5 Description of Organizational Food Sites............................................103

Table 3.6 Distinctions Between the Qualitative Aims.........................................108

Table 3.7 Scoring for Organizational Context....................................................109

Table 4.1 Descriptive Characteristics of Organizations and Respondents ...........118

Table 4.2 Food Environment Policies Among Organizations ...............................119

Table 4.3 Respondent-Specific Facilitators of HFEP Implementation..................123

Table 4.4 Respondent-Specific Barriers to HFEP Implementation .......................127

Table 5.1 Baseline Characteristics of 17 Food Venues Pre-SSB Ban .................139

Table 5.2 Percent Change in Gross Sales Among Beverage Categories ..............142

Table 5.3 Stratified Revenue Differences, by Covariate ........................................143

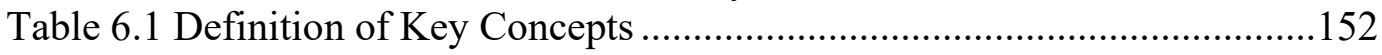

Table 6.2 Operationalization of Organizational Context......................................153

Table 6.3 Scoring for Organizational Context......................................................155

Table 6.4 Organizational Characteristics of Study Participants ...........................156

Table 6.5 Healthy Food Environment Policies Across Organizations ..................157

Table 6.6 Organizational Contexts of Health Care Institutions .............................159

Table 6.7 Supporting Documentation for Contextual Determinations .................160 


\section{LIST OF FIGURES}

Figure 1.1 Adapted Rogers' Innovation-Decision Model...................................19

Figure 2.1 Ecological Model of the Socioenvironmental Food Environment.......29

Figure 3.1 Organizing Framework for Interview Guide Development ................92

Figure 5.1 Monthly Gross Beverage Revenue, Jan 2012-Nov 2019, ................140

Figure 5.2 Fitted Lines for Monthly Gross Revenues, Pre and Post SSB Ban....141 


\section{GLOSSARY}

ACA

AHA

$\mathrm{CDC}$

COREQ

Food Environment

Health Care Organization

$\mathrm{HCWH}$

Healthy Food Environment Policies (HFEPs)

HFEPI

HHI

HHFI

Implementation
Affordable Care Act

American Hospital Association

Centers for Disease Control and Prevention

Consolidated Criteria for Reporting Qualitative Research

The collective physical, economic, policy, and sociocultural surroundings, opportunities, and conditions that influence people's food and beverage choices and nutritional status.

Any organization in the health care industry that is consumer facing, including hospitals, clinics, medical offices and state health agencies. Insurance companies, hospice homes, and education centers are excluded.

Health Care Without Harm

Practices that improve retail food environments by instituting any combination of the following: (1) trans-fat and/or sodium limits; (2) limiting or banning access to sugar-sweetened beverages; (3) instituting serving size limits; (4) prioritizing healthy methods of food preparation; (5) nutrition labeling for meals and items at point of service; (6) promotion of healthy foods and beverages in marketing materials; (7) using pricing strategies to increase the affordability of healthy items; (8) healthy vending policies; and (9) other HFEPI practices.

Healthy Food Environment Policy Index

Healthier Hospital Initiative

Healthy Hospital Food Initiative

A deliberately initiated attempt to introduce new, or modify existing, patterns of action in health care or some other formal organizational setting. Deliberate initiation means that an intervention is: institutionally sanctioned; formally 
defined; consciously planned; and intended to lead to a changed outcome.

\section{INFORMAS}

Institutions

IRB

ITSA

JCAHO

NSLP

Obesogenic Food

Environment

Organizational Context

SSBs

SRA

WHO
International Network for Food and Obesity/NonCommunicable Disease Research, Monitoring and Action Support

Rules, norms, practices and relationships that influence individual and collective behavior. Institutions at one level can also shape activity at another, establish the types of venue where policy decisions are made, and make the rules that allow particular types of policy actors to enter.

Institutional Review Board

Interrupted time series analysis

Joint Commission on Accreditation of Healthcare Organizations

National School Lunch Program

An environment within which nutrient-poor and energy dense foods saturate the collective physical, economic and sociocultural conditions that influence nutritional status.

The aggregate of human and non-human characteristics that comprise an organization, including structure (governance, size, interorganizational coupling), receptivity (tension for change, norms and culture), resource availability (slack resources, social network location, information sharing), and leadership (change agents, stakeholders, vision).

Sugar-sweetened beverages

Segmented regression analysis

World Health Organization 


\section{PREFACE}

Chapters 4, 5 and 6 of this dissertation were organized in the conventional journal article format. Known as a hybrid dissertation model, this format exchanges the traditional narrative summary of the study results with three standalone papers. After the successful completion of the doctoral study, these chapters will be submitted to peer-reviewed journals in the fields of implementation science and organizational theory. Given this article format, there will be some redundancy in these chapters with preceding chapters in terms of methodology and literature review. 


\section{CHAPTER 1: INTRODUCTION}

Rates of overnutrition and obesity in the United States have escalated dramatically throughout the twentieth century, resulting in an ongoing obesity epidemic. While the causal pathways of obesity development are complex, a major driver of this public health crisis is obesogenic food environments, characterized by nutrient-poor and energy-dense foods that saturate the collective physical, economic and sociocultural conditions that influence nutritional status (Hall, 2018; Swinburn et al., 2015). Thus, improving food environments is critical in order to increase the availability, affordability and access of healthy foods and beverages available for consumption (CDC, 2019; Rodgers et al., 2018). Though there is no one panacea to this growing crisis, populationlevel interventions aimed at enhancing the food environment offer a promising start in addressing this epidemic (Stevens et al., 2017; Vandevijvere et al., 2015; Sallis et al., 2009).

Food environments in organizations such as hospitals and public health agencies warrant special consideration given their health-focused mission. Though health care systems are emblematic of health and healing, there is often a lack of healthful retail options available to the consumer (Reed \& Chenault, 2010). As concerns regarding limited healthy food availability have grown, health care systems have faced criticism over contracting with fast food franchises to supply retail offerings (Cram et al., 2002). In response to this criticism, health-focused organizations across the nation have begun to increase the nutritional value of food and beverage offerings within their sites, 
recognizing their influence over dietary patterns and consumer behavior (Moran et al., 2016; Guthrie et al., 2015; van Hulst et al., 2013; Kolasa et al., 2010). Improving food environments in hospitals has been highlighted by the Centers for Disease Control and Prevention (CDC) as one of seven key strategies to prevent and manage obesity (CDC, 2015). As these organizations impact patients, employees, clients and visitors through public and medical services, a healthy food environment can offset downstream social and health costs associated with obesity and align with institutional values. Further, since over half (55.7\%) of adults in the US receive health insurance coverage through their employers (Abramowitz \& O’Hara, 2017), retailing healthy menu items indirectly benefits such organizations through decreased health spending for employee medical costs and insurance premiums.

However, like many organizations, hospitals and public health agencies are sociotechnical organizations that are nested within diverse health care environments (e.g., rural-urban communities; differences in payer mix, patient populations and tax status). Further, these complex organizations are also constantly evolving in response to changing policy landscapes (Meyer \& Scott, 1992). With limitations such as imperfect information and constraints on time, facing an external threat as ambiguous as the obesity epidemic can evoke the strategy of "doing nothing", also known as organizational inertia (Hannan \& Freeman, 1984; Coiera, 2011). If and when a particular strategy is considered, it must typically align with professional norms and values (Lawrence, 1999), as societal and institutional pressures often shape the kind of strategies undertaken by organizations to address external pressures (Oliver, 1991). Though implementation of healthy food 
environment policies within health care organizations are promising activities that are growing in popularity, little is known about organizational factors that may facilitate or inhibit effective uptake.

Healthy food environment policies (HFEPs) are defined as those practices taken by organizations that improve food composition, labeling, marketing and accessibility (e.g., competitive pricing, convenient locations) (INFORMAS, 2014). The concept of HFEPs was first introduced as indicators of the Healthy Food Environment Policy Index, which describes a spectrum of activities taken by private and public entities to foster healthier food environments. Examples of HFEPs can include nutritional labeling of foods, removing sugar-sweetened beverages, reducing unhealthy food promotion, setting nutritional thresholds for vendors and providing operational guidance on the purchasing practices of healthy foods (Vandevijere et al., 2015). The governing principles of the Healthy Food Environment Policy Index were borne out of the International Network for Food and Obesity/Non-Communicable Disease Research, Monitoring and Action Support (INFORMAS), sponsored by the World Health Organization (INFORMAS, 2014; Swinburn et al., 2013). These practices represent structural efforts that aim to make healthy food and beverage procurement affordable and available.

This chapter begins with a description of the obesity epidemic, examines the institutional role of health care organizations in modeling healthy behaviors, and describes how implementation of HFEPs within these organizations represent a strategy of aligning food environments with the health-focused missions and values of the health care field. This will be followed by the problem statement, the key research question and 
aims of the study, along with a discussion of the theoretical and conceptual frameworks that provide the foundation for this research. A discussion of the purpose and significance of the study concludes this chapter.

\section{Background}

The United States spends an estimated $\$ 149.4$ billion on obesity and obesityrelated expenditures per year, representing $9.1 \%$ of the country's gross domestic product (Kim \& Basu, 2016). However, this distribution of costs and resources is not uniform across the nation, with obesity and obesity-related health care costs ranging from $\$ 768$ per person in Oregon to $\$ 279$ per person in Wyoming (Wang et al., 2015). This unequal distribution of costs is overshadowed by the high medical and social costs of obesity: increased morbidity, early mortality, and lower quality of life (Jia et al., 2005). Obesity also affects psychological wellbeing, with higher rates of depression and suicidality among persons with obesity (Mather et al., 2009). From an occupational standpoint, obesity is associated with decreased productivity, increased sick days, and increased short-term disability and permanent disability (Van Nuys et al., 2014; Durden et al., 2008). Prior to the enactment of the Affordable Care Act (ACA), health insurers used body mass index of prospective insureds to set premium rates, resulting in unequal access to and affordability of care among persons with overweight and obesity and higher subsequent health spending (Heinen \& Darling, 2009).

Fundamentally, obesity is caused by long-term excess energy, influenced by biological, environmental and sociocultural influences that have profound influences on health (Sallis, 2009; Bray \& Popkin, 1998; Hall, 2018). Within these influences are 
multiple levels of risk, including at the intra- and inter-personal, neighborhood, and policy level (Afshin et al., 2014). Given these complex influences and multi-level risks, the obesity epidemic is a "wicked" public health problem (PLoS, 2013), with evolving determinants and hard-to-define solutions.

\section{The Institutional Role of Health care Organizations in Modeling Health}

Symbolically, health care organizations are considered "houses of healing, open to the community" (Heinen \& Darling, 2009, p. 115), acting as role models within society to shape perceptions about healthy behaviors (Estabrook, Zapka \& Lemon, 2012). Decisions made by these organizations affect not only internal constituents but also broader audiences. Additionally, these organizations play a key role in the development and dissemination of new knowledge and evidence-based policy and practice. For example, when the health consequences of smoking became evident, hospitals modeled piecewise steps to prohibit indoor smoking. First, hospitals restricted the sale of cigarettes from onsite shops (Lesser, Cohen \& Brook, 2012). Next, hospitals began requiring smoking cessation as part of preoperative workup for many procedures. This was followed by designating outdoor smoking areas and, ultimately, banning smoking outright on hospital campuses (Freedhoff \& Stevenson, 2008). These practices rippled into communities and worksites in a similar stepwise fashion, subsequently contributing to a downward trend of smoking prevalence among Americans (Drope et al., 2018). This example is one of many that demonstrates the institutional leverage that hospitals wield in shaping public perception and modeling healthy behaviors for broader audiences and settings. Similarly, as diet-related chronic diseases grow at an unsustainable rate, there is 
mounting pressure for health care institutions to signal the need for widescale change by modeling healthy food environments.

From a public health perspective, institutional actors within the health care field have employed diverse strategies in addressing the obesity epidemic, ranging in levels of influence. Upstream, midstream, and downstream approaches — characterized as solutions addressing social determinants, modifying behavior change, or refining treatments, respectively—vary in effectiveness (Rutter et al., 2017). "Downstream" examples include hospital systems establishing best practices within allopathic obesity care, state Medicaid programs covering pharmacologic therapies for overweight and obesity, and health insurers expanding coverage of obesity treatment modalities (Sacks et al., 2009). "Midstream" efforts of health care organizations, particularly state health agencies, often involve strategies encouraging personal responsibility for individual health (e.g., public service announcements/campaigns) and increasing consumer knowledge of food and beverage purchases (e.g., calorie labeling in restaurants, front-of-packaging disclosures). Finally, examples of "upstream" public health efforts include lobbying efforts by medical and public health groups for tighter regulatory environments (e.g., advertising restrictions to young persons, limits on food and beverage portion sizes) and state health agencies collaborating with employers to offer worksite wellness initiatives (e.g., subsidized gym memberships, weekly farmer's markets) (Kumanyika, 2007). Health care organizations also coordinate with governmental and community organizations to facilitate practiceand evidence-based research studies (Sacks et al., 2009; Huang \& Glass, 2008), refining the knowledge base on obesity prevention initiatives. 
Though there are a number of strategies that health care organizations have undertaken to address the obesity epidemic, it is noteworthy that many of these same organizations do not have healthy food environments - Champ and colleagues (2019) report that the majority of food items sold in government-run hospitals are candy, soda and fried foods, items that strongly contradict government-sponsored recommendations. Malhotra (2013) describes the marketing strategies of the "junk food industry" that have infiltrated hospitals wards. Lawrence and colleagues (2009) found that $79 \%$ of California hospitals primarily sold soda and candy in their vending machines. Among Canadian and US hospitals with accredited pediatric residency programs, most were found to have suboptimal food environments, with commercial vendors such as Starbucks, Burger King, and McDonald's present in a third of the hospitals surveyed (McDonald et al., 2006). A third study surveyed parents' opinions of hospital food and reported that $95 \%$ responded that hospital food outlets should provide mostly healthy items and that such items should have clear signage (Bell et al., 2013). Given that many chronic diseases today are diet-related, providing food environments that are high in nutrient density is critical. As centers of health and healing, hospitals and health care agencies inevitably shape perceptions around which foods are fit for consumption—Sahud and colleagues (2006) found that parents associated the presence of fast food establishments within hospitals more positively, ascribing healthiness to fast food items. The impact of healthful food and beverage availability cannot be understated: there is a positive association between the quality of the food environment and a lower prevalence of obesity (Moore et al., 2008; Morland et al., 2006). Lesser and colleagues (2012) 
demonstrated the role marketing strategies within hospital cafeterias played in shaping food purchases. Food availability directly impacts consumption profiles (Caspi et al., 2012; CDC, 2003; Bodor et al., 2008), such that maintaining healthy food environments is essential to changing structures around health, illness, and disparity (Kumanyika, 2005).

Given the poor food environments found in most health care organizations, there is increasing demand to change these environments (Wojcicki, 2013). The Healthier Hospitals Initiative (HHI, 2013), a joint project of Health Care Without Harm and Practice Greenhealth, and the Hospital Healthy Food Initiative (HHFI, 2014), which is a Partnership for a Healthier America program, are two nationally-run platforms that invite hospitals to reexamine the pivotal role of food environments and pledge to institute changes to enhance these environments. Smaller-scale, regional programs include the Commons Health Hospital Challenge (Minnesota) (ISF, 2014), Healthy Beverages in Hospitals Campaign (Boston) (BPHC, 2016), Healthy Hospital Food Initiative (New York City) (Moran et al., 2016), and the Karat Gold Partnership (Kansas City) (KGP, 2018), all of which enumerate principles that participating health care systems pledge to adopt. Of these programs, the HHI collaborative has emerged as the most widely-used resource among hospitals dedicated to enhancing onsite food environments due to its emphasis on resource sharing, webinars and metrics (HHI, 2013). As of 2020, over 70 participating hospitals and health care systems have joined HHI. Additionally, the National Conference of State Legislatures has highlighted the role of state agency food environments in addressing the obesity epidemic, encouraging public agencies to adopt 
HFEPs within consumer-facing settings (Pomeranz, 2011). These resources signal the growing awareness of organizations - across industries — on the role of the food environment and its impact.

\section{The Case for HFEPs within Health Care Organizations}

HFEPs represent a population-level strategy to combat obesogenic food environments, possessing a number of attractive qualities, or "innovation attributes" (Bordenave, 1976), that support their implementation within many contexts. In considering innovation attributes, however, one must also consider the "innovationsystem fit", which is defined as the interaction between an "innovation" and its potential context (Greenhalgh et al., 2004). The innovation-system fit for implementing HFEPs within health-focused organizations is particularly high given that both the proposed innovation and context share the same goal of health promotion. Innovation-system fit, as a construct, underscores that innovations must be considered situationally and not on the basis of their standalone attributes. Plsek (2003), in discussing innovation spread among complex health care systems, cautions that organizational context is an often-overlooked element. Examined contextually, HFEPs align food environments with the professional and health-focused missions of health care institutions and thus represent a salient strategy for such institutions when addressing the obesity epidemic.

For this study, HFEPs were defined as any combination of the following policies instituted within the retail environment: (1) trans-fat and/or sodium limits; (2) restricting 
the sale of sugar-sweetened beverages; (3) instituting serving size limits; (4) prioritizing healthy methods of food preparation (e.g., removing fryers); (5) nutrition labeling for meals and items at point of service; (6) promotion of healthy foods and beverages in marketing materials; (7) using pricing strategies to increase the affordability of healthy items; (8) healthy vending policies; and (9) other HFEPI practices. The wide breadth of inclusion in this definition was intentional: in casting as wide a net as possible, this definition captures most, if not all, contemporary efforts by health care organizations aiming to enhance retail food environments. Some of these policies are structural by nature and change the food landscape, availability and affordability, while others are more individual-level — these differences in scope will be examined during the analysis. However, for purposes of capture, this wide-reaching definition was constructed. These specific policies were selected given their application in both the grey and refereed literature, which is reviewed in detail in Chapter 2.

An additional advantage of HFEPs is that, as opposed to other obesity prevention strategies that may require significant resources and/or are radical in their scope, HFEPs can be applied in a stepwise fashion. Depending on the comprehensiveness of the approach (e.g., marketing policies, pricing strategies), HFEPs can be applied to relevant operational processes within the organization and actors would be able to assess and amend activities as needed. HFEPs reinforce sociocultural norms of healthy foods within health care organizations, emphasizing a culture of health within health care. As these organizations exist to promote health, HFEPs enhance the food environment as well as 
shape the sociocultural norms regarding which foods are desirable for consumption (McGuire, 2012; Larson \& Story, 2009; Ashe et al., 2011).

Despite the increase of how-to guides and implementation toolkits to improve hospital environments (CDC, 2015) or calls for the health care sector to promote healthy foods and beverages (AHA, 2012; PHLC, 2013), most of the academic literature examining HFEPs within health care organizations focuses on the inpatient dietary environment (Marshall et al., 2012; Pedersen et al., 2012; Brantley, 2009), leaving a paucity of information on facilitators or barriers of implementation within the retail food environment that serves employees, outpatients, and members of the public. One study reviewing HFEPs within Los Angeles county organizations concluded that while utilization of these practices had increased, "much remains unknown about their context, the processes required to implement them effectively, and the factors that facilitate their sustainability" (Robles, Wood, Kimmons \& Kuo, 2013, p. 191).

Whereas innovation and diffusion research efforts attempt to broadly appeal to a wide audience, organizational context represents a need for custom-tailored solutions and case-by-case considerations; thus, context is a problem for implementation science (Glisson \& Schoenwald, 2005; Plsek, 2003). The absence or presence of certain organizational factors can result in distinct opportunities or challenges to changing the food environment. For example, the size of an organization or its degree of centralization may be a barrier in garnering enough buy-in for the decision to adopt HFEPs; conversely, a well-connected health care system can learn from associated systems and sidestep known implementation challenges. Additionally, the perceived and actual costs of 
implementing HFEPs may differ based on an organization's information channels or organizational culture. Perceived costs may under- or over-estimate the actual effort needed and the degree to which these factors play a real role in decision-making and implementation vary in influence (Birken et al., 2015).

The "costs" of search, information, and negotiation are also known as transaction costs (Williamson, 1989). Transaction costs differ among organizations given their resource availability, communication channels, and resource constraints, meaning that both startup commitments as well as implementation processes will differ from one organization to the next. In their systematic review of diffusion of innovations within organizations, Greenhalgh et al. (2004) caution that innovations whose perceived benefits are outweighed by perceived costs are unlikely to be implemented, regardless of the actual costs and benefits. Porter, Allen \& Angle (2003) submit that organizations strive to implement solutions that have the greatest (perceived) benefit for the lowest cost. However, the "costs of search," coupled with environmental uncertainty and ambiguous external pressures, create a terrain that require significant upstart resources in order to search, select, and commit to a strategy (Lubell et al., 2017; Birken et al., 2015).

The application and evaluation of HFEPs within organizations in the health care sector is limited, focusing on one or two policies or food sites (Palmedo \& Gordon, 2019; Hartigan et al., 2017; Lessard et al., 2014; Eneli et al., 2014; Bell et al., 2013; Block et al., 2010). The extent to which effects on revenue or consumer behavior are observed in the retail health care food environment is also limited and would be useful in evaluating the consequences of these practices. Implementation of HFEPs is becoming increasingly 
highlighted in mainstream news media - notable organizations such as Google (Nestle, 2015), Twitter (Lev-Ram, 2015), Microsoft (Barberio, 2017), and WeWork (McGregor, 2018) have instituted wide-reaching HFEPs. Thus, evaluating the process and outcomes of HFEPs can aid prospective organizations that are considering which practices to adopt.

This background underscored the importance of utilizing population-level approaches to address the obesity epidemic and the institutional role of health care organizations in modeling healthy contexts and behaviors. HFEPs represent a populationlevel policy that health care organizations can implement to align food environments with organizational values. While a number of obesity prevention practices exist, the innovation-system fitness of HFEPs within health care organizations is robust, which makes studying the implementation factors and evaluating outcomes of this policy worthwhile.

\section{Statement of the Problem}

In 2011, the chair of the American Hospital Association urged hospitals to eliminate unhealthy foods and beverages in cafeterias, stating that fast foods communicated an "inconsistent message" to the broader community (AHA, 2012). Implementation of HFEPs has emerged as a promising strategy that organizations can adopt to structurally enhance food environments. However, the pathway for implementation has not been straightforward, requiring a better understanding of the system and policy factors that may inhibit or facilitate successful implementation. Further, as HFEP implementation may look different across organizations, the degree to which the organizational context may play a role in the kinds of implementation 
challenges experienced is unknown. Thus, a better understanding of the relevant organizational factors affecting implementation can enhance uptake among organizations as well as inform diffusion strategies. Finally, though HFEPs are increasing in popularity among organizations across all industries, there remains limited evidence regarding these practices' effects on short- and long-term revenue impact and consumer behavior.

\section{Research Question}

Organizations continually utilize evolving strategies to address internal and external challenges (March \& Simon, 1993). As health care organizations function dually as health care providers and employers, obesity is doubly burdensome to these organizations through increased employee health costs as well as decreased worker productivity (Wang et al., 2015; Dee et al., 2014). Thus, studying obesity prevention policies that can be deployed within these organizations is of two-fold interest. Identifying factors associated with successful implementation as well as detailing how organizational characteristics (operationalized later in the chapter) create environments in which a factor can be a barrier in one context and a facilitator in another would be more helpful for organizations than a single "how-to" guide or toolkit. Further, evaluating the outcomes of HFEP implementation, such as changes in consumer behavior or financial impact, would help prospective decisionmakers choose a feasible strategy.

This study sought to answer the following question: How does the organizational context of health care organizations affect the implementation process and economic outcomes of healthy food environment policies? The study utilized a mixed methods study design and had three aims. 
Aim 1 sought to qualitatively describe the barriers to and facilitators of implementing HFEPs in six health care organizations: (1) a state public health agency; (2) a regional not-for-profit health plan and delivery system; (3) a tier-1 academic medical center; (4) a federally-run medical center; (5) a faith-based hospital network; and (6) a rural hospital. This first aim had three objectives: (a) to describe the implementation factors that inhibited the implementation process; (b) to describe the implementation factors that facilitated the implementation process; and (c) to compare and contrast the implementation factors cited between operational and executive leaders of participating organizations.

Aim 2 sought to quantitatively evaluate the revenue and consumer behavior outcomes of a sugar-sweetened beverage (SSB) sales ban, a HFEP that has been growing in popularity and uptake. This HFEP was implemented within a not-for-profit health care system and was chosen for two reasons: first, the researcher was granted access to the relevant financial data associated with the HFEP; second, the SSB ban was implemented at a specific point in time, January 2015, enabling a quasi-experimental design. Aim 2 had three objectives: (a) to compare monthly gross sales of all beverage categories before (FY 2012-2014) and after (FY 2015-2019) the sugar-sweetened beverage ban; (b) to assess the percent change in sales by beverage type before (FY 2012-2014) and after (FY 2015-2019) the sugar-sweetened beverage ban; and (c) to describe the stratified impact of the ban on venues that differed by size, type and geographic location. These findings described the economic impact of a HFEP initiative (objectives (a) and (c)) as well as 
changes in consumer behavior (objective (b)), adding to a sparse literature that evaluates short- and medium-term outcomes of these kinds of practices.

Finally, Aim 3 sought to determine the organizational contexts of the six participating institutions. This final aim had three objectives: (a) to determine the organizational context for each organization; (b) to describe the relationship between contexts and HFEPs adopted for each organization; and (c) to develop context-informed policy recommendations for HFEP implementation. The aim of producing contextinformed policy recommendations was to detail how organizational characteristics (structure, resource availability, etc.) create environments in which a factor could be a barrier in one context or a facilitator in another. By detailing the implementation process of six distinct health care organizations, the summary would be a resource for participating as well as prospective organizations to understand how factors can serve as facilitators or barriers depending on the host organization and outline steps future organizations can take to determine their readiness and anticipate implementation challenges based on their characteristics.

\section{Theoretical Framework}

"The medical profession was influential in reducing smoking in the United States; it has the capacity to encourage food-system change within its own institutions" (Lesser, Cohen \& Brook, 2012, p. 984).

The decision to focus on health care organizations as the implementation setting for this study had more to do with moral authority and obligation than the pragmatic role that hospitals play in society. As health care organizations consider strategic responses to 
address the obesity epidemic, institutional theory (DiMaggio \& Powell, 1983) provided a lens for evaluating strategies on the basis of innovation-system fit (Greenhalgh et al., 2004). Specifically, Powell \& DiMaggio's new institutionalism (2012) provided the general theoretical foundation for this study.

Unlike Weber, who framed organizations as rational self-interested actors and emphasized bureaucracy $(1978 ; 2015)$, new institutionalism argues that organizations operate in open environments that influence, and are influenced by, a network of similarly-situated organizations (Thompson, 1967; Katz \& Kahn, 1978). Aligned with the open rational systems theory (Scott, 1987), organizations and environments are engaged in a feedback loop where both resources and challenges are collectively shared and experienced (Meyer \& Rowan, 1977). Through the lens of DiMaggio \& Powell's new institutionalism, health care organizations implement innovative solutions to problems that are viewed as legitimate within their environment (March \& Olsen, 1975). Further, organizations tend to favor inaction, known as organizational inertia, when external pressures are ambiguous and complex and organizations typically remain in this inertia until the point at which external pressures become unavoidable (e.g., the opioid crisis, obesity epidemic). Additionally, when organizational goals are unclear when responding to external threats, institutional theory posits that organizations will mimic the strategies of similarly-situated organizations (Powell \& DiMaggio, 2012). This behavior is known as mimetic isomorphism and highlights the dependent nature of organizations on their environment and neighboring organizations (DiMaggio \& Powell, 1983). Engaging in prescriptive and suitable behavior, known as complying with "rationalized myths" 
(Meyer \& Rowan, 1977), underscores the extant force of institutionalization on influencing organizational strategy. This contrasts with the assumption that organizations are closed-system rational actors; rather, these institutions are complex systems that have limited information, possess hazy goals, and act to conform with societal expectations (Jones, 2003; Meyer \& Rowan, 1977; March \& Simon, 1993). Given these conditions, this theoretical framework provided a useful lens through which to examine how health care organizations are grappling with the obesity epidemic and how HFEPs represent a legitimate and appropriate strategy.

\section{Conceptual Framework}

With new institutionalism providing the theoretical basis for this work, an adaptation of Rogers' Model of Five Stages in the Innovation-Decision Process (2003) provided the conceptual framework through which to study the implementation process of HFEPs as well as the impact on consumer behavior and revenue to Aim 2's organization. The decision to use a well-cited framework from the field of implementation science was intentional in order to adequately capture the process of application. The model's emphasis on context was apt given Aim 3's focus on organizational context. As stated by May and colleagues (2016), internal organizational conditions are becoming increasingly reflected in implementation frameworks.

This adapted model addressed the main elements of the study and conceptualized how the environmental context (e.g., professional norms, public opinion, external pressures), HFEPs (e.g., pricing strategies, market promotion), and organizational context (structure, resources, culture and leadership) were inputs in determining the adoption 
decision. These input factors also affected the process of implementation in that the broader environment, specific strategies employed, and organizational context shaped which organizational factors facilitated or inhibited HFEP implementation. Outcomes, namely changes in revenue and consumer behavior, were a direct result of the implementation process (Figure 1.1).

\section{Figure 1.1 Adapted Rogers' Innovation-Decision Model}

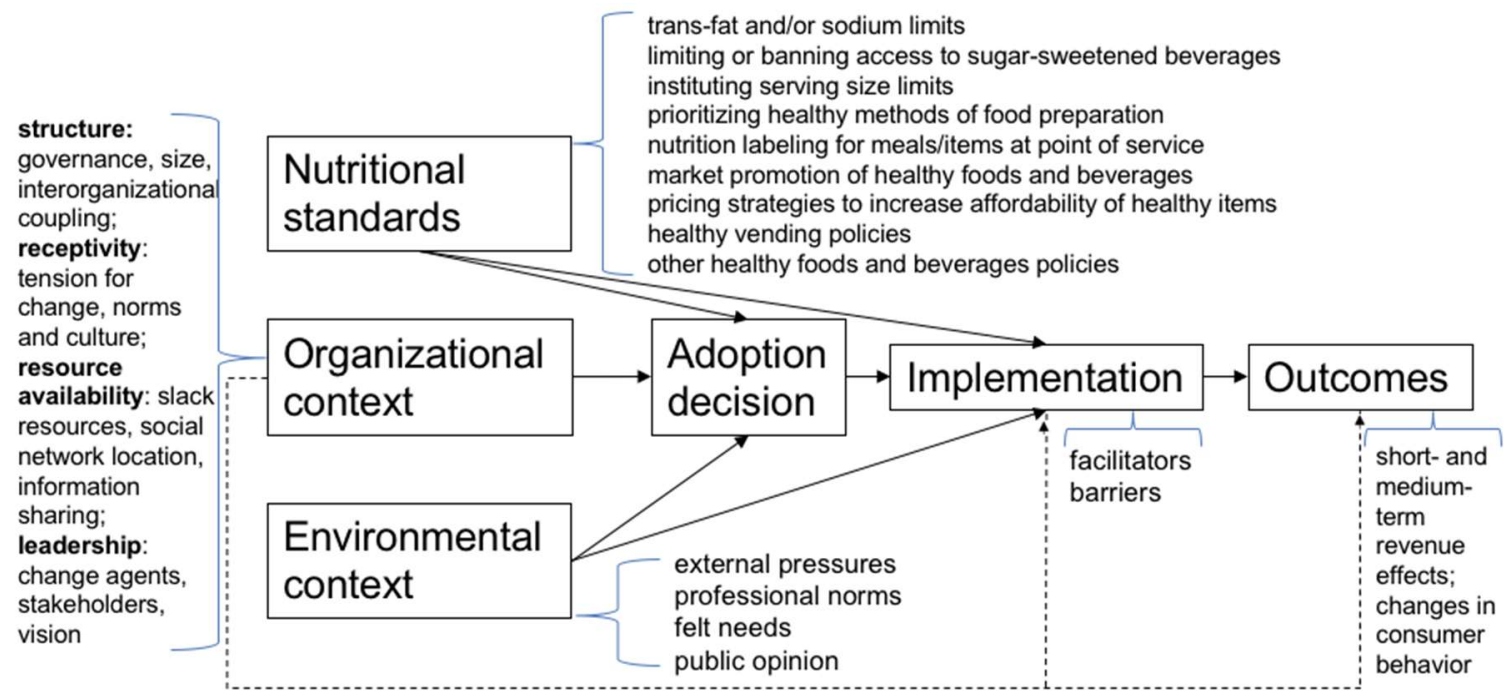

As HFEPs and their implementation were best understood when they were examined contextually, this model's emphasis on external (environmental) as well as internal (organizational) inputs made this a well-suited model with which to study all aims.

Examining Rogers' adapted framework through an institutional lens, health care organizations would be affected by external environmental pressures - in this case, the obesity epidemic and growing criticism regarding poor food environments — and would respond by incorporating appropriate and "legitimate" (March \& Olsen, 1975) solutions 
to address these pressures. As discussed previously, HFEPs have a number of inherent characteristics that prime their uptake within health care organizations: they align with the professional values of health care (e.g., promoting and delivering health); their adoption would result in an incremental, as opposed to radical, change within these organizations; and a number of health care systems have begun implementing rigorous HFEPs, signaling to similarly-situated organizations that this is one of many appropriate solutions to an ambiguous and complex epidemic (HHI, 2013).

As this dissertation work sought to understand how organizational factors affected the implementation of HFEPs, a number of organizational characteristics were of interest to the study, including structure (governance, size, interorganizational coupling), receptivity (tension for change, norms and culture), resource availability (slack resources, social network location, information sharing), and leadership (change agents, stakeholders, vision). These terms are operationalized in Chapter 2. With this framework anchoring the study, and institutional theory providing the theoretical grounding, a robust analysis of the implementation factors was accomplished while acknowledging the role of the organizational context.

\section{Purpose and Significance of the Study}

This research and its findings drew on the insights of regional health care leaders to examine strategies related to improving food environments. The multiple environmental and organizational factors affecting the selection of the type of obesity prevention strategy, as well as the implementation strategy, may be a daunting task for 
any given organization - even more so if there is no dedicated personnel, time, or unit that absorbs this responsibility (Bradley et al., 2004; Slappendel, 1996). Though organizations within the health care industry faced the same competitive and fiscal pressures that exist in any other field, the added perceived responsibility of modeling healthy behaviors and providing healthy environments (Reed \& Chenault, 2010) made this dissertation work particularly useful. This study also allays some of the perceived and actual startup costs associated with adoption of these practices since it described the implementation process of six distinct organizations - prospective organizational decision-makers can glean from the experiences of the organization that most closely resembled its own organizational context in order to anticipate potential barriers and facilitators. Finally, this study utilized an adapted model of Rogers' Five Stages in the Innovation-Decision Process_-given that the study utilized a widely-known model that had not yet been applied to guide the implementation of HFEPs, this was an added contribution to the fields of implementation science and dissemination research.

\section{Summary}

Addressing the obesity epidemic through structural interventions is increasing in popularity as the medical and social costs of this disease continue to grow. As health care organizations explore obesity prevention strategies, practices that are perceived as legitimate and that align with institutional norms and values will be adopted. HFEPs represent a structural, evidence-based strategy to enhance the food environment. Understanding the policy and organizational factors will enhance implementation strategies as well as account for variations in organizational context in order to anticipate 
implementation challenges. In Chapter 2, a review of the relevant literature is presented to inform the study design and rationale. 


\section{CHAPTER 2: REVIEW OF THE LITERATURE}

This chapter reviews the relevant literature pertinent to this dissertation. The review included the following:

1) An overview of the obesity epidemic, including historical framing and drivers, as well as contemporary obesity prevention strategies aimed at enhancing the food environment;

2) A brief review of healthy food environment policies in the U.S. and their application in the literature, including revenue and consumer behavior outcomes;

3) An examination of the role that health care institutions play in addressing the obesity epidemic, including a description of new institutionalism, its core concepts, and relevance to health care food environments;

4) A discussion clarifying the concept of organizational context, including a description of key organizational elements, and the degree to which organizational context influences implementation success; and

5) A concluding summary of the reviewed literature, synthesizing the historical, theoretical and conceptual bases for this work.

\section{The Obesity Epidemic: Framing, Drivers, and Prevention Strategies}

Nearly 150 billion dollars are spent every year in the U.S. on treating obesity and obesity-related medical expenditures (Kim \& Basu, 2016). Obesity is defined as 
excessive accumulation of fat in the body (Welcome, 2019). There are multiple methods to measure obesity (e.g., abdominal circumference, bodyfat percentage, skinfold thickness); however, the most commonly-used measure of body composition is the body mass index (BMI), dividing a person's weight (in kilograms) by height (squared in meters) to calculate a ratio. A BMI ratio $\left(\mathrm{kg} / \mathrm{m}^{2}\right)$ between 25 and 30 indicates overweight whereas BMI equal to or greater than $30 \mathrm{~kg} / \mathrm{m}^{2}$ indicates obesity.

The urgency for addressing this disease is warranted given the significant impact on morbidity and mortality. Psychosocial correlates of obesity include anxiety, depression, and suicidal ideation (Sarwer \& Polonsky, 2016; Katz et al., 2000). Average life expectancy as well as fertility is diminished among those with a BMI $\geq 30 \mathrm{~kg} / \mathrm{m}^{2}$ (Fontaine et al., 2003; Jokela, Elovainio \& Kivimaki, 2008). From a productivity standpoint, occupational absenteeism (work absences due to poor health) and presenteeism (diminished engagement/productivity) are also widely reported in people with obesity (Andreyeva et al., 2014; Finkelstein et al., 2010). These examples underscore how obesity can affect both the economic as well as social fabrics of communities. Thus, the ability to prevent obesity, especially earlier in the life course, can prevent a wide array of negative outcomes.

Framing of Obesity

From a framing perspective, the cultural construction of obesity as an individualized disease has resulted in prevention and treatment interventions that focus on the individual instead of on whole populations (Barry et al., 2011; Gordon-Larsen et 
al., 2006). In addition to focusing on the individual level, the characterization of obesity as a behavior-related condition has resulted in policymakers endorsing behavior-focused policies, such as recommendations for better diets and increased exercise (Brownell et al., 2010). Further, the medicalization of obesity as a clinical disease of biological origins, not a societal expression of inequality (Krieger \& Smith, 2004) or an outcome of globalization (Hawkes, 2006), has had a lasting impact on what constitutes appropriate treatment for obesity.

In 2013, the American Medical Association officially recognized obesity as a disease (Kyle et al., 2016). Prior to this decision, obesity was largely considered a risk factor for diseases such as diabetes, hypertension, sleep apnea, cardiovascular disease, and cancer. After this recognition, obesity could be listed as the primary clinical diagnosis instead of as a secondary risk factor for a related condition. A host of pharmaco-therapeutic and surgical interventions for the treatment of obesity followed (Kim et al., 2014). These outputs of the medical model, a framework that is often criticized for its scientific reductionism and a theoretical commitment to individualism (Chang \& Christakis, 2002), has further allayed political decision-makers from legislating structural changes to affect population health. Instead, physicians and dieticians have been framed as experts on the matter and subsequent efforts have been cast toward developing clinical interventions to remedy this epidemic (Blackburn, 2011).

As a result of these phenomena, public perceptions about obesity tacitly affirm that obesity is a product of individual choices, exacerbating the attribution of individual responsibility and increasing stigmatization and discrimination among persons with 
obesity (Brownell et al., 2010). Medicalizing and individualizing obesity has also resulted in negative consequences for the framing of accountability to address this public health crisis - a global epidemic has been ostensibly explained away as widespread weakness of will (Hofmann, 2016). The imperative to acknowledge the ideological underpinnings of disease causation unearths implicit biases about the nature of obesity and, subsequently, the rationale for preferring one scope of action to another when constructing treatment and prevention approaches (Tesh \& Tesh, 1988; Funk et al., 2016).

\section{Drivers of the Obesity Epidemic}

The growing trends of obesity onset have affected every social, economic and geographic population group (Jaacks et al., 2019). In order to conduct a meaningful overview of the obesity epidemic, exploring the assumptions about the causes of obesity is necessary. Though this epidemic is multifaceted, prevention efforts are often singular in their approach, often treating the "symptoms" of this epidemic (PLoS, 2013).

Fundamentally, obesity is caused by long-term excess energy, influenced by biological, environmental and sociocultural influences that have profound influences on health (Sallis, 2009; Bray \& Popkin, 1998; Hall, 2018). Within these influences are multiple levels of risk, including at the interpersonal, neighborhood, and governmental level (Huang et al., 2009; Afshin et al., 2014). Concepts such as food security (Dinour et al., 2007) and neighborhood walkability (Creatore et al., 2016) underscore that structural forces shape energy balance. Layered onto these structural forces are sociocultural influences, such as cultural norms regarding portion sizes (Berg \& Forslund, 2015) and 
workplace norms of working through the lunch hour (Wolfe, 2007). Thus, to frame energy balance as the result of individual "choices" is to make a willful decision to ignore the broader context within which individuals are placed. Further, ignoring broader norms and influences can lead to perceptions that stoke stigma and discrimination against individuals who develop obesity (Spahlholz et al., 2016).

Historical phenomena can also underscore the complexity of obesity causation. For example, the concepts of "urban sprawl," "bedroom communities," and "the suburb" have led to widespread reliance on automobiles to travel between work, school and home (Frankston, 2003). Industrial progress throughout the twentieth century has led to an increased reliance on mechanized automation and technological innovations among most Americans, enabling lifestyles that require little physical activity to function (Swinburn, Egger, \& Raza, 1999; Swinburn et al., 2011; Yanovski \& Yanovski, 2018). This generational rise in sedentarism has led to the creation of the concept of obesogenicity, defined as the promotion of energy conservation through normalization of energy-saving appliances and activities (Townsend \& Lake, 2017). For example, innovations such as the laundry machine and dishwasher have decreased the energy threshold needed for household maintenance (Lanningham-Foster, Nysse \& Levine, 2003). Similarly, mobile banking, online grocers and the general digitization of essential activities has further reduced the energy requirement to meet basic needs (Vandelanotte et al., 2009).

Obesogenicity can be observed in the built environment in workplaces, entertainment venues, and retail establishments (Hall, 2018; Townshend \& Lake, 2009). Garfinkel-Castro and colleagues (2017) contend that the built environment's influence on 
physical activity corresponds to the scale of urbanization: as most people typically live in urban environments, transportation and zoning infrastructures prioritize vehicular movement and conservation of physical energy. Regarding causality, studies examining the built environment typically report correlations and associations with energy expenditure instead of making causal claims (Ding \& Gebel, 2012). A meta-systematic review of 36 reviews identify methodological rigor as the main limitation when examining built environment influence on energy expenditure (Ding \& Gebel, 2012). Further, the profit motives of nongovernmental groups when creating spaces are typically not aligned with the goals of population health and are also hard to oversee and govern (Swinburn et al., 1999). Combined with industrial and technological progress, the physical and cultural environments can explain much of why daily energy expenditure has rapidly diminished over a relatively short period of time (Swinburn et al., 1999).

\section{Major Driver: Food Environments}

Notwithstanding the aforementioned drivers of obesity, the unmatched ability of American corporations to mass-produce and mass-market convenience foods has been the most notable hallmark of obesogenic environments (Hall, 2018; Spence et al., 2016; Roberto et al., 2015; Lake \& Townshend, 2006). The proliferation of nutrient-poor and energy-dense foods stems from changes enacted in US farm bills beginning in the 1970s, namely financial incentives for commodity crops. Also known as cash crops, commodity crops are defined as crops that are primarily grown for trade in the international market instead of for domestic consumption (Keeney \& Kemp, 2004). The most commonly- 
grown commodity crops in the US are corn, wheat and soy. These incentives dramatically accelerated food production, increased portion sizes and introduced sweeteners (such as high fructose corn syrup) into every category of foods (Rodgers et al., 2018). Since these changes, adult obesity prevalence has swelled from $13 \%$ in 1960 to $40 \%$ in 2016 (Hales et al., 2017). Further, the affordability of processed foods has led to a disproportionate rise in obesity among poor households (Moran et al., 2019; Farrell et al., 2017; Drewnowski \& Specter, 2004) and communities of color (Jevitt, 2019; Neff et al., 2009; Freeman, 2007). The excess production of corn, and later corn syrup, has introduced an unparalleled availability of sugar-sweetened beverages into schools, worksites and commercial spaces (Khadaee \& Saeidi, 2016; Lobstein et al., 2015; Hu, 2013; Forshee, Anderson, \& Storey, 2008).

Figure 2.1 presents an anchoring framework for discussing how multiple levels of the food environment influence obesity development through influences on dietary behaviors.

\section{Figure 2.1 Ecological model of the socioenvironmental food environment}

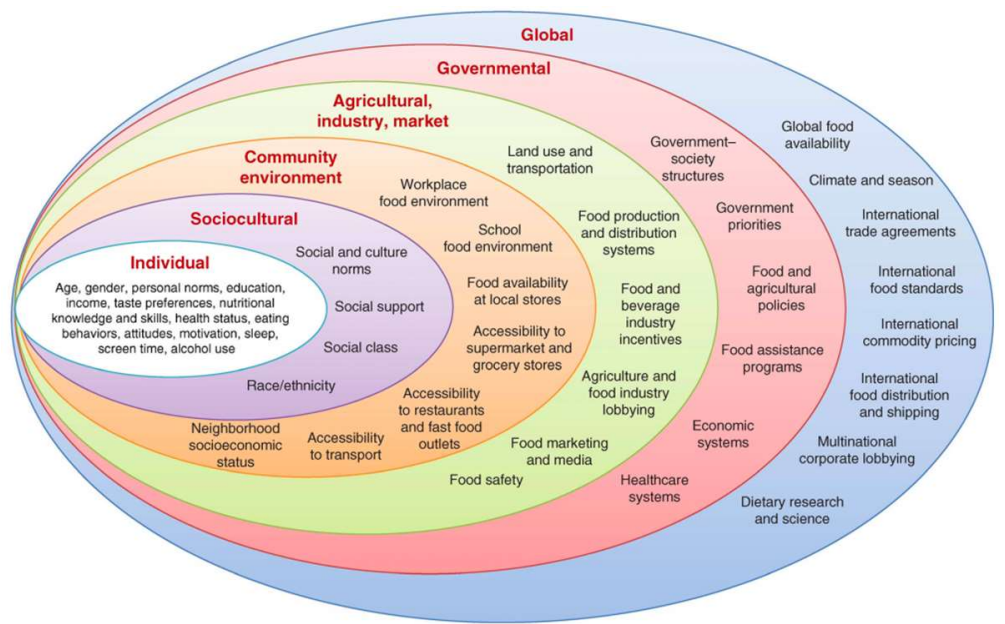

Source: Afshin et al., 2014 
Beginning at the individual level, certain characteristics favor or protect against obesity development — a higher educational attainment corresponds with a lower risk of obesity onset (Cohen, Rai, Rekhhopf \& Abrams, 2013), screen time is associated with higher BMIs (Boone et al., 2007), and stress can increase one's susceptibility to obesity onset (Sinha \& Jastreboff, 2013). The added layers of phenomena such as underestimation of caloric intake (Chandon \& Wansink, 2007), binging (Klatzkin et al., 2018), "grazing” (Carter \& Jansen, 2012), emotional eating (Kemp, Bui \& Grier, 2013) and overall nutrient literacy (Arabin et al., 2019) can provide additional insights individual-level influences on frequency and type of food consumption (Wansink \& Chandon, 2006). Further, while the genetic and epigenetic nature of obesity development is prima facie individual level, progress in the field of Developmental Origins of Health and Disease (DOHaD) demonstrates that one's propensity for obesity can be strongly influenced by factors such as maternal obesity, maternal stress, and adverse in-utero conditions (Lohman et al., 2009; Vickers et al., 2007). Originally known as Barker's "fetal origins" hypothesis of low birth weight and susceptibility of later-life chronic disease (Barker, 1998; De Boo \& Harding, 2006), the intergenerational influences makes clear that prevention efforts should be made for living populations as well as to ameliorate causes of intergenerational obesity.

The fields of precision medicine and bioinformatics research have demonstrated how specific genes and loci can be linked to monogenic and polygenic obesity (Paschou et al., 2016; Munoz Yanez et al., 2017; Choquet \& Meyer, 2011). Genome-wide association studies continue to shed light on the molecular basis of obesity, with 
syndromes such as Prader-Willi and Bardet-Biedl elucidating the intergenerational nature of obesity (Thorleifsson et al., 2009; Scuteri et al., 2007; Bell et al., 2005).

The individual level is often the first, and oftentimes only, dimension considered for obesity prevention efforts in the US (Nestle \& Jacobson, 2000). Common aims of such efforts are to increase information, change attitudes, or influence personal norms and habits (Barry, Brescoll \& Gollust, 2013). The emphasis on changing individual behavior through fear tactics (Bailey, Wang \& Kaiser, 2018), empowerment (Puhl et al., 2013), or information sharing (Boles et al., 2014) overlooks the multiple layers through which dietary choices are made. Individualized obesity prevention approaches are those efforts that try to change individual behavior without addressing the broader context within which these behaviors exist. This kind of approach reinforces that the obesity epidemic is caused by poor individual behaviors and that if people make "good choices," this will be sufficient in reversing the trends of obesity.

Transitioning to the next level, sociocultural influences on food choice have been cited as drivers of poor food choices (De Ridder et al., 2013; Young \& Nestle, 2002), as cultural mores often promote unhealthy foods and portion sizes (Isoldi et al., 2012). Religious and ethnic conventions, as well as family traditions, may shape the kinds of ingredients, food preparation methods, and mealtime duration (Kumanyika et al., 2012; Caprio et al., 2008; Bruss, Morris \& Dannison, 2003). Furthermore, influences such as regional/geographic location and family size may shape decision contexts regarding diet (Wang et al., 2007). Socially, food is a medium through which people strengthen familial and community bonds (Neely, Walton, \& Stephens, 2014). However, despite the positive 
benefits to socialization, social eating has been associated with overconsumption of food (Kemp, Bui \& Grier, 2013) as well as increased snacking (Piernas \& Popkin, 2009). As food serves many functions beyond providing nourishment, sociocultural context both shapes and is shaped by norms regarding food consumption (Strahan et al., 2007; Miller \& Halberstadt, 2005).

Accessibility of healthy and affordable food is highly dependent on physical location and resources. Regarding the community level, the ongoing obesity epidemic in the United States has been associated with environmental factors such as the proliferation of unhealthy foods in schools and neighborhoods (Zick et al., 2009; Andreyeva et al., 2008) as well as promotion of unhealthy foods in marketing and advertising (Sahud et al., 2006). Land use measures, such as walkability and availability of public green spaces, as well as the built environment are cited as important predictors of obesity across sex and race/ethnicity (Frank, Andresen \& Schmid, 2004). The role of food and beverage industries in mass-marketing foods with low nutrient density is higher in low-income communities and in communities of color (Jevitt, 2019; Neff et al., 2009).

School food environments represent a cornerstone environment for $99 \%$ of American children aged 7-13 (Hall \& Lannoy, 2015), which provide more than half of all daily calories (Woo \& Taveras, 2014). Schools represent a setting where socialization and conditioning of eating behaviors take place and where children, cued and reinforced by their peers, form deeply ingrained assumptions and behaviors around food. School food environments have been well-reviewed in the literature (Bevans et al., 2011; Snelling \& Kennard, 2009; Van Cauwenberghe et al., 2010; Whatley Blum et al., 2007; 
Jaime \& Lock, 2009) and the availability of competitive foods - unregulated low-nutrient foods sold alongside federally reimbursable school meals - has been a large driver of poor nutrition among students at all grade levels.

The presence of these low-nutrient foods in schools has increased over the past forty years and correlates with rising childhood obesity rates, constituting what Moodie and colleagues (2006) call a "market failure." The Healthy Hunger Free Kids Act of 2010, a community-focused prevention effort, was passed by Congress with bipartisan support to implement Institute of Medicine (since renamed the National Academy of Medicine) recommendations to the USDA's school meal standards (USDA, 2014). Since implementation of this legislation, total breakfast participation has increased by almost $25 \%$, there is $16 \%$ more vegetable consumption, school lunch revenue has increased by almost \$200 million, and students report a higher level of satisfaction with school meals (USDA, 2014).

Worksite food environments have also been reviewed extensively (Wolfe, 2007; Mhurchu, Aston \& Jebb, 2010; Finkelstein et al., 2010; Anderson et al., 2009; Pratt et al., 2007). Almeida and colleagues (2014) report that the presence of a cafeteria and fewer vending machines and kiosks positively influenced eating habits of employees. Wolfe (2007) extensively characterizes contemporary workplace norms, such as working through the lunch hour, consuming high-fat foods in response to high stress environments, and placing a premium on convenience when selecting lunch purchases.

When considering influences at the agricultural, industry, and governmental levels - and without delving into the minutiae of the complex system that is American 
farming - consumers eat what farmers grow, and what farmers grow is steered by state and federal agricultural policy. Given the generous and well-guarded federal and state subsidies regarding corn, wheat, rice, milk and soybean agricultural interests, it may serve as an explanation as to how the processed foods industry, especially large-scale actors, has amassed its political and economic capital (Berry, 2015). Financial incentives, originally meant to safeguard farmers from bankruptcy during economic downswings, have transformed into guaranteed income for farmers (Keeney \& Kemp, 2004). Quotas, "price floors" and buy-back programs are all attributes of a suite of agricultural programs that have ballooned federal subsidies to over $\$ 20$ billion annually (Smith, 2017). In addition to receiving subsidies to fund crop farming, the federal government also funnels excess crop production into food assistance programs (Smith, 2017). There are over sixty federally-funded aid programs for agricultural interests within eight categories (in descending order by aid amount): crop insurance, agricultural risk coverage, price loss coverage, conservation programs, marketing loans, disaster aid, export promotion, and research (Edwards, 2018). These agricultural and governmental activities inevitably shape which foods are most affordable and available in the United States.

\section{Summary}

Though a number of obesity causation theories have been offered, from genetic causation to a rise in sedentarism, this review underscores the immense role and influence of the food environment on shaping individual- and population-level dietary choices and subsequent risk for developing overweight and obesity. The location of this study's aims 
lies in the community level—by studying the application and evaluation of HFEPs, these practices can be disseminated among health care organizations and beyond.

\section{Contemporary Obesity Prevention Strategies Aimed at the Food Environment}

Hospital systems, state agencies, and health care organizations have begun to address this public health crisis through concerted policymaking efforts aimed at enhancing the food environment (Sisnowski et al., 2017). The following prevention efforts will be examined for their effects to date.

Nutrient and Menu Labeling Embraced by many states, the practice of requiring chain restaurants to provide caloric and nutrient information has emerged as a popular obesity prevention tool. Depending on the state, this policy typically affects food vendors that have more than ten commercial locations in order to avoid adversely affecting smaller restaurants and food vendors (Hill et al., 2010). Proponents of this practice posit that affected vendors will likely start offering healthier items in order to minimize negative press and retain their consumer base (Lee et al., 2008; Engelhard, Garson, \& Dorn, 2009). As part of the ACA, menu labeling was required for chain restaurants with more than twenty locations (Cusick, 2011). Similar to menu labeling, public health officials have supported changes in nutritional labeling, a required retail component for most foods sold in the US. Types of nutritional labeling efforts can include requirements to standardize of package labels (either back-of-package or front-of-package) to promote consumer awareness and require larger fonts for calorie-per-serving labeling to increase visibility of nutrient information (Silverglade \& Heller, 2010). Another labeling effort 
requires prominently displayed nutrient information for meals that include toys for children (Levi et al., 2012).

The logic guiding these strategies is rooted in the economic tenet that increased information will lead to better decision-making (Arrow, 1973). Consumers, aware of the nutritional makeup of an item, will be in a better position to make informed decisions regarding their diet. Further, standardization of menu- and nutrition-labeling has been cited to increased nutrition literacy (Sharf et al., 2012). However, many of these efforts rely on the critical assumption that most Americans possess a basic level of knowledge regarding the recommended daily allowances for fat, sugar, salt, among other nutrients (Parker, Ratzan \& Lurie, 2003). A working knowledge of daily allowances and nutritent needs is known as nutrient literacy or health literacy. A systematic review of nutrition and health literacy reported that the majority of nutritional materials are written at the ninth-grade level whereas $20 \%$ of the population reads at a fifth-grade level (Carbone \& Zoellner, 2012). Further, another systematic literature review by Swartz, Braxton \& Viera (2011) concluded that menu-labeling neither altered consumer behavior nor brought about a reduction in calories consumed.

\section{Food Assistance Programs The Supplemental Nutrition Assistance Program} (SNAP) and the Special Supplemental Nutrition Program for Women, Infants and Children (WIC) are two examples of food assistance programs that offer low-income families benefits to purchase grocery items through means testing. Those individuals and family that are eligible are required to re-certify their eligibility and most programs are funded federally with individual states contributing funds. Programs like SNAP have 
implemented a number of innovative obesity prevention strategies, such as including targeted subsidies to incentivize healthful food purchases (Shenkin \& Jacobson, 2010) or encouraging vendors at local farmers' markets to accept program vouchers as payment for fruits and vegetables (Cutter et al., 2009). Changes to SNAP and WIC guidelines have also been made to nudge enrollees in making more nutrient-dense purchases (BCBS, 2010), including one discontinued policy which prohibited the use of SNAP benefits to purchase sugar-sweetened beverages (Brownell \& Ludwig, 2011). There is growing evidence that incentivizing healthful food purchases is more effective (and less controversial) than banning unhealthy items (Blumenthal et al., 2014). Hastings and colleagues (2019) have stated that making SNAP purchase data more available from the FDA will enable robust analysis regarding the program's effect on nutrition and health. A central repository of purchasing information would enable policymakers and researchers to further refine the links between tested interventions and their effect on purchasing behavior and food consumption.

Additionally, in order to increase access to healthy foods and beverages, financing mechanisms have been instituted by state and federal programs. Two examples include Healthier Food Retail (HFR) and Healthy Food Financing (HFF) initiatives (USDT, 2019). HFR policies have the goal of improving the retail food environments of grocery stores and farmers' markets to offer healthier choices to consumers to improve diets. Similarly, HFF initiatives aim to finance grocery stores and other healthy food retailers in underserved urban and rural communities to enhance access to healthful food and beverage options. HFR initiatives have been enacted in 12 states since 2001 (USDT, 
2019). These financing efforts typically coordinate with food assistance programs, such as the Supplemental Nutritional Assistance Program, to subsidize costs.

Food and Beverage Tax Strategies Neoliberal economics contend that price elasticity can be an important determinant of purchase. Anchored with this lens, food and beverage tax strategies rely on the rationale that raising prices on select nutrient-poor food and beverage items will decrease market demand and ultimately improve population health (Cornelsen et al., 2015; Mytton et al., 2012; Khan et al., 2012; Brownell \& Frieden, 2009; Andreyeva, Long, \& Brownell, 2010). Foods and beverages that are often proposed to be taxed often have no nutritional value, such as sugar-sweetened beverages or candies (Friedman, 2010; Chriqui et al., 2007; Antos et al., 2009). State policymakers have historically proposed directing revenue from food and beverage taxes into funds dedicated for obesity prevention efforts (Frieden, Dietz \& Collins, 2010; Engelhard, Garson \& Dorn, 2009). However, a noteworthy trend in response to food and beverage taxation has been the passage of preemption laws (Pomeranz, Mozaffarian \& Micha, 2017), which are defined as state-level legislation measures that restrict local governments from passing any food-related regulations or restrictions. Given that the majority of food and beverage taxation measures are legislated through local venues, preemption laws can virtually eliminate local political activity in this realm. As of 2019, 12 states have passed some variation of a preemption law, ranging from narrow restrictions such as forbidding local legislation regarding calorie labeling in restaurants to wide-sweeping prohibitions such as forbidding any local authority action aimed at nutrition, food policy, or farming legislation (Pomeranz et al., 2019). 
Additional legal and regulatory policies regarding the food environment can prevent obesity. The Chilean government has exercised remarkable regulatory levers in addressing their unprecedented levels of childhood and adult obesity (Perez-Escamilla et al., 2017; Gregori et al., 2017). Since 2016, marketing restrictions, mandatory front-ofpackaging redesigns, and food and beverage taxes have been implemented to address the local community food environments. Additionally, legislation has included child protection amendments to Chile's constitution, resulting in prohibitions in food advertising directed at children less than 14 years of age (Corvalan et al., 2019; Boyland \& Harris, 2017).

Public Service Announcements The public service announcement (PSA) has been the historical medium by which government agencies and advocacy organizations disseminate knowledge and evidence-based practices regarding a range of public health topics. PSAs attempt to increase information, change attitudes, or influence personal habits. PSAs can also take the form of counter-marketing campaigns, showcasing the detrimental health impacts of unhealthy behaviors (Allen et al., 2015; Wallace et al., 2007). For example, one information campaign used fear tactics to deter consumption of sugar-sweetened beverages (Jordan et al., 2012; Witte \& Allen, 2000). From providing nutritional information to advertising local farmer's markets, PSAs can take the form of a wide array of messaging media, which relies on targeted and recurrent messaging to positively influence individual behavior surrounding diet and activity (Farley et al., 2017). 
Another type of a public service announcement is Choose MyPlate, the current nutrition guide published by the USDA Center for Nutrition Policy and Promotion and an update of the food pyramid that was originally endorsed by the USDA. Since 2011, this guide provides dietary recommendations, dividing an illustrate dinner plate into four categories consisting of $30 \%$ grains, $40 \%$ vegetables, $10 \%$ fruits, and $20 \%$ protein alongside an eight-ounce glass of dairy milk (Willet, 2017). Upon its release, Choose MyPlate garnered criticism from the academic community, especially in regard to the allocations for meat and dairy (Willett, 2017), with the chief complaint stating that the USDA was beholden to meat and dairy interests. The Harvard School of Public Health releases a competing version of Choose MyPlate that has reduced grain content, water instead of milk, and a higher ratio of vegetables to fruits (Datz, 2011).

Workplace Strategies Employer-sponsored wellness programs, which include a broad category of policies (Ammendolia et al., 2016), often include changes to internal food environments to improve workforce health and lower occupational disease. While employers have historically been responsible for the prevention of occupational hazards, the responsivity of personal health, including avoiding overweight and obesity, has been primarily placed on employees. The role of employers has evolved to encompass larger preventive efforts aimed at morbidity, with access to nutrition classes, nutritionists and corporate dietary guidelines (Schulte et al., 2007; Schulte, Wagner \& Downes, 2008; Story et al., 2008; Nyberg \& Olsen, 2010).

Agricultural Policy. Agricultural policies shape the selection, investments, and distribution of food production in the United States and globally. For almost 50 years, the 
US Farm Bill has financially prioritized the production of commodity crops, namely corn, wheat and soy. As the obesity epidemic has emerged, there has been increasing support to increase federal funding for fruit and vegetable production (Krueger, Krub \& Hayes, 2010), as the current costs of production of fruits and vegetables have a lower margin than commodity crops (Wallinga, 2010). One policy initiative proposed to remove fruit and vegetable planting restrictions in commodity food programs, which serve mainly low-income, elderly, or vulnerable populations (Wallinga, 2010). Yet another policy goal is to provide training, loans, research and marketing support to fruit and vegetable farmers (Lee et al., 2008). Finally, another policy proposal has called for the creation of a fruit and vegetable subsidy program (Barnes, 2010). Currently, surplus commodities produced under Farm Bill programs are subsequently purchased by the federal government and rerouted into child nutrition programs, such as the National School Lunch and Breakfast programs - by subsidizing fruits and vegetables, there is enhanced likelihood that surplus production of these crops will also be utilized in these programs. These efforts are essential for farmers to remain economically competitive (Krueger, Krub \& Hayes, 2010).

\section{Healthy Food Environment Policies: Historical Development and Application}

The link between nutrition and obesity is not new (Stunkard \& Penick, 1979). As

the obesity epidemic grows larger by the decade, public and private institutions that vend foods face certain decisions when purchasing and selling foods. In the United States, HFEPs have most often been released through federally funded activity or as part of a 
professional association's concerted effort (Nestle \& Jacobson, 2000). These organizations typically institute procurement strategies by establishing thresholds for calorie, sugar, sodium, and trans-fat content for foods as well as other strategies, including but not limited to organic foods certification, local procurement, portion limits, pricing strategies, marketing techniques, and product placement (Brambila-Macias et al., 2011; French, 2003; Robles, Wood, Kimmons \& Kuo, 2013; Story et al., 2008).

To address the growing awareness of the relationship between food environments and population health, the US Department of Health and Human Services and General Services Administration developed and released Sustainability Guidelines for Federal Concessions and Vending Operations (Kimmons et al., 2012; CDC, 2012) and these standards have been a starting point for almost all HFEPs evaluated in the literature (Story et al., 2008; Ashe, Graff \& Spector, 2011). In 2010, these federal agencies established standards that maximized healthier and sustainable food options provided by vendors to governmentally funded facilities. Another well-cited set of nutritional standards are the CDC's Food Service Guidelines for Federal Facilities, representing a set of voluntary best practices (Onufrak et al., 2016). In addition to requiring specific benchmarks to be met, such guidelines typically also provide contractual standards for vendors and signal changes in market demand.

Among 19,500 municipalities in the US, approximately 3.2\% report having written nutrition guidelines (Onufrak et al., 2016). Municipalities with such guidelines are likely to be in urban areas ( $>50,000$ people), located on the west coast, and include provisions for low-calorie beverages and increased fruits and vegetables. Less than $1 \%$ 
include pricing strategies for healthy foods (Onufrak et al., 2016). Within Oregon, two sets of nutritional guidelines exist at the municipal level, the first being within the City of Portland's Community Centers and Pools (adopting the "Healthy Snacks Standards") and the second being within Multnomah County's Food Services for Correctional Facilities (adopting the Dietary Guidelines for Americans) (Hackett \& Rhoads, 2010).

Though operational criteria are not a novel mechanism in food purchasing, proliferation of such criteria in order to enhance food environments and promote health is new (Kimmons et al., 2012; CDC, 2012; Story et al., 2008). Implementation of HFEPs is becoming a popular strategy within large employers, such as Google (Nestle, 2015), Twitter (Lev-Ram, 2015), Microsoft (Barberio, 2017), and WeWork (McGregor, 2018). One review of HFEP application concluded that while utilization of these practices had increased, "much remains unknown about their context, the processes required to implement them effectively, and the factors that facilitate their sustainability, especially in such broad and diverse settings as schools, county government facilities, and cities" (Robles, Wood, Kimmons \& Kuo, 2013, p. 191).

\section{Definition for Study}

For this study, HFEPs were defined as any combination of the following policies instituted within the retail environment: (1) trans-fat and/or sodium limits; (2) restricting the sale of sugar-sweetened beverages; (3) instituting serving size limits; (4) prioritizing healthy methods of food preparation (e.g., removing fryers); (5) nutrition labeling for meals and items at point of service; (6) promotion of healthy foods and beverages in 
marketing materials; (7) using pricing strategies to increase the affordability of healthy items; (8) healthy vending policies; and (9) other practices described in the HFEPI.

The wide breadth of inclusion in this definition was intentional: in casting as wide a net as possible, this definition captured most, if not all, contemporary efforts by health care organizations aiming to enhance retail food environments. Some of these policies were structural, changing the food landscape, availability and affordability, while others were more individual in focus - the degree to which individual- versus population-level policies were more effective in creating conducive environments for healthy purchasing was examined as part of Aim 3. However, for purposes of capture, this wide-reaching definition was constructed. The emphasis on these specific policies was due, in part, to their uptake in the grey and referred literature, which was reviewed in the following section.

Review of Healthy Food Environment Policy Application

Each of the nine types of policies that were included in the HFEP definition for this dissertation work were selected based on a review of the grey and refereed literature of contemporary strategies that health care organizations have instituted in the past decade. The following review of initiatives, policies and activities underscore the nuanced fashion by which organizations implement similar policies in different ways. Some health care systems implement one or two policies, such as restricting sugarsweetened beverages or providing nutritional labeling, while others implement multicomponent initiatives. Still others have joined collaborative partnerships with advocacy 
groups, adopting standard principles and adapting them over time to their institutional structures.

Trans-Fat and Sodium Limits In alignment with the CDC's Food Service Guidelines (Onufrak et al., 2016), setting limits on sugar, salt, trans fat and other nutrients that vendors can supply has been a strategy among institutions committed to enhancing food offerings. In 2018, Geisinger initiative a nutritional program that eliminated trans-fat from all food offerings (Stender, 2018). Similarly, in 2017, University of Michigan Medicine set nutrient limits, including trans-fat, fiber, added sugars, sodium, and total fat (MHealthy, 2017). In 2012, Children's Mercy Kansas City (CMKS) joined the Partnership for a Healthier America, adopting numerous health commitments including nutrient thresholds for meals (Friedman, 2012). New food menus included three daily "wellness meals" that were $<700$ calories, 0 grams of trans fat, $<10 \%$ calories from saturated fat, and $<800 \mathrm{mg}$ of sodium.

Sugar-Sweetened Beverage (SSB) Restrictions In 2018, the University of Pennsylvania Health System pledged to remove SSBs, including sports drinks and juices, from across all hospital campuses (Hunton, 2018). This is a part of a greater healthy food environment initiative that recognizes the role of hospitals in creating healthy spaces for patients, employees and the public. Geisinger, similarly, recently announced an SSB ban (Stender, 2018). The Cleveland Clinic stopped vending SSBs throughout their vending machines and cafeterias since 2010, warning of the effects of added sugars on health (CDC, 2012). Since 2012, all facilities part of the Baylor Health Care System, headquartered in Dallas, Texas, eliminated SSBs from cafeterias and vending machines, 
offering water, fruit juices and diet drinks instead (Baylor, 2012). Similarly, four Vanguard Hospitals located in Chicago, Illinois, have restricted sale of beverages that contain more than a teaspoon of sugar per 12-ounce serving (Eng, 2012). Children's Mercy Hospitals and Clinics, as part of their wellness initiative, stopped selling SSBs in 2013, offering water, diet drinks and fruit juices instead (Kansas City Star, 2015). Rady Children's Hospital began a Rethink Your Drink campaign that included an SSB ban (Hartigan, 2017). The Commons Health Hospital Challenge, a healthy dining initiative in Minnesota, contains a pledge to remove SSBs from dining rooms, cafeterias, vending machines and onsite food courts - seven Minnesota and Wisconsin hospitals and health systems joined this commitment in 2014 (ISF, 2014). In 2013, eleven ProMedica hospitals in Ohio and Michigan stopped selling SSBs within all sites (PHLC, 2013).

Serving Size Limits The University of Michigan, as part of "MHealthy Criteria," has instituted serving size limits for baked goods, breads, pre-packaged snacks and main dishes sold (MHealthy, 2017). Food offerings in compliance with the limit have a MHealthy logo that communicates to the consumer at the point of purchase.

Healthy Methods of Food Preparation In 2012, the Cleveland Clinic replaced fryers with ovens in hospital cafeterias (CDC, 2012), as did CMKS in 2013 (Kansas City Star, 2015), Indiana University Health in 2013, Seattle Children's Hospital in 2014, the University of Michigan in 2017, Geisinger in 2018 (Stender, 2018), and all hospitals a part of the Hospital Healthy Food Initiative (HHFI, 2014). These health systems have all removed deep fryers from their food preparation facilitates and instead offer baked or steamed options in place of conventionally fried foods. 
Nutrition- and Calorie-Labeling As of 2018, there were 50 health systems that were part of Collaborative for Healthy Weight, which requires nutrient- and calorielabeling of all foods and beverages sold on premises (Fernandes et al., 2016). Similarly, 700 hospitals, through the Partnership for a Healthier America, had committed to several healthy policies within their food sites, including calorie-labeling of cafeteria and inpatient meals (HHFI, 2014). Hospitals including CMKS (Kansas City, MO) and Geisinger (Danville, PA) have joined this initiative.

Healthy Foods Marketing Approximately 700 hospitals and health systems that have joined Partnership for a Healthier America have pledged to market healthy foods in advertisements and promotional material (HHFI, 2014). Further, a number of hospitals that have joined the Collaborative for Healthy Weight have a "healthy check out" policy, which restricts unhealthy items from being within five feet of all cash registers (Fernandes et al., 2016). A popular marketing strategy has also been stoplight labeling, which color-codes items as green, yellow or red to indicate the healthiness of the offering (Sacks et al., 2009). For example, University of Pennsylvania Health System places green stickers on healthy items and actively engages in product placement for healthy foods. Similarly, nine hospitals in the Boston, MA, region use stoplight labeling as well as product placement to promote nutritious items (BPHC, 2016). Thorndike et al. (2019) examined the effects of a hospital cafeteria program that adopted "traffic light" food labels and found a $6.2 \%$ decrease in calories per transaction over two years.

Pricing Strategies In 2011, Tufts Medical Center implemented a pricing policy with beverages sold onsite, requiring that water be priced lower than other beverage 
options across all vending and retail locations. Conversely, Faulkner Hospital took the approach of increasing the price of SSBs relative to water prices. Both techniques use pricing to favor water purchases.

Healthy Vending Policies Lessard and colleagues (2014) studied a healthy vending program instituted within three state agencies in Delaware, finding that the intervention had neutral effects on revenue. In 2018, the University of Pennsylvania Health System adopted the Balanced Choice vending policy, which graded the nutrient density of vending machine choices and assigned stoplight labeling (green, yellow, red) — green for healthy options — and required that 30\% of options meet "green" nutritional requirements. Since 2012, 22 hospitals in New York City have implemented snack vending standards.

Other Methods of Increasing Access for Healthy Foods and Beverages As part of an employee wellness initiative, Tufts Medical Center in Boston, MA, instituted a selfnamed "merchology" approach to retail food sites, studying consumer habits and purchasing patterns and adjusting sales and pricing techniques to promote healthier options. Another popular approach among health systems has been terminating fast food leases from health care sites. Beginning in 2010, Lurie Children's Hospital (Chicago, IL), Parkland Health (Dallas, TX), Truman Medical Center (Kansas City, MO) and Vanderbilt Medical Center (Nashville, TN) have all terminated McDonald's locations from their sites (Franklin, 2010). 


\section{Reported Outcomes: Changes in Revenue and Consumer Behavior}

Reviewing HFEP application and evaluation in the literature yielded a number of studies that reported outcomes on revenue impact, behavior change, and changes in anthropometric measures. The majority of evaluations were conducted within school settings (Bevans et al., 2011; Snelling \& Kennard, 2009; Schwartz et al., 2009; Van Cauwenberghe et al., 2010; Whatley Blum et al., 2007; Jaime \& Lock, 2009) and worksite settings (Mason et al., 2014; Jaskiewicz et al., 2013; Blanck et al., 2013). These evaluations focused primarily on changes in dietary intake and behavior, though some report on financial impact. A literature search focusing on HFEPs within health care organizations yielded six relevant studies that either focused on one policy or retail site (Palmedo \& Gordon, 2019; Hartigan et al., 2017; Lessard et al., 2014; Eneli et al., 2014; Bell et al., 2013; Block et al., 2010) -all other evaluations focused on in-patient dietary standards. Thus, in addition to reviewing the six studies, application of HFEPs within other settings, namely school food environments and worksites, were reviewed. These evaluations were organized by outcomes reported: revenue effects and changes in dietary behavior were presented along with reported facilitators and barriers in the implementation process of HFEPs.

\section{Revenue Implications}

Determining the extent to which HFEPs have revenue impacts on the host organization will help hospital administrators and executive leadership anticipate consequences. Eleven studies and two systematic reviews reported revenue effects 
associated with HFEP implementation. Brown and Tammineni (2009) reported that advertising efforts, attractive pricing and portion changes were all associated with increased profits for school-based vending sales. Grech \& Allman-Farinelli (2015) conducted a systematic review on nutritional interventions and vending machine sales in schools, reviewing twelve studies. They report that those interventions that employed pricing strategies, namely reducing prices of healthy offerings, increased sales of healthy products. They conclude that pricing strategies are effective tools to increase sales of healthy items.

Wharton, Long \& Schwartz (2008) examined school revenue changes in a systematic review examining four refereed and three state-based reports. The authors cite that concerns of revenue loss were a persistent fear among implementation sites, especially among school administrators, but that there were few data to substantiate those fears. Interestingly, an incidental benefit was observed through increased participation in the National School Lunch Program (NSLP), which produced a net positive financial benefit. In a 3-year study detailing revenue trends of school HFEPs, the authors reported increased sales and participation in the NSLP (Cohen et al., 2016). Long, Henderson \& Schwartz (2010) also cited increased reimbursement from NSLP activity in their study examining incentives. Another study examined the financial impact of school nutrition policies and determined that revenue trends were net neutral two years postimplementation (Wojcicki \& Heyman, 2006).

French and colleagues studied the effects of peer-based promotion of healthy foods on sales of healthier offerings (French et al., 2004; French et al., 2001), finding that 
instituting peer-based food education alongside HFEPs had no adverse effects on a la carte revenue sales. Separately, another study examining pricing strategies within vending machines concluded that no significant differences were observed in vending machines despite increased purchased of subsidized lower-fat items (French et al., 1997), indicating that the subsidy was compensated through increased purchases. The Arizona Department of Education facilitated a pilot study testing food that met certain nutritional criteria (Arizona Dept of Education, 2005), and reported that no loss of revenue was observed six months post-implementation. California, similarly, piloted HFEPs within school settings and reported mixed results, reporting an overall net increase in revenue despite losses incurred at several schools (CWH, 2005). Another study in California sought to determine the effect of legislated HFEPs on sales and student consumption at 99 schools (Woodward-Lopez et al., 2010). The authors reported an initial decrease in school food sales but increased participation in the meal program, as did Long and colleagues (2013) in a study associating a state statute with an increase in school lunch participation.

\section{Behavior Implications}

HFEPs represent a population-level strategy of enhancing the food environment; anticipating changes in consumer behavior and demand can aid operational staff. Four studies and one systematic review considered behavioral outcomes of school and worksite HFEPs. Snelling and Kennard (2009) evaluated HFEPs within the school food environment, focusing on adolescent dietary intake before and after implementation. 
Results showed that HFEPs contributed to a positive shift in the nutrient density of foods consumed. Similarly, a systematic review of European school-based interventions promoting healthy diets included 42 studies that evaluated effects on dietary behavior and/or weight change (Van Cauwenberghe et al., 2010). Strong evidence of effect was found for multi-pronged interventions that promoted vegetable intake. Educational interventions had limited evidence of positive effects, and adolescents were the least likely demographic to respond positively to nutritional interventions. Further, the authors concluded that few studies included anthropometrics, a limitation for evaluating effectiveness. However, the few studies that did report body weights suggested positive relationships between measures and policy goals (Fung et al., 2013). Additionally, HFEPs as part of worksite nutrition policy had demonstrated modest improvements in employee BMI (Gabel et al., 2009).

Food and nutrition policies improved the school food environment, yielding positive effects on the availability and consumption of healthy foods (Jaime \& Lock, 2009). Researchers studying the school food environment evaluated the effects of nutritional guidelines that promoted low-fat, low-sugar foods. The authors found that the proportion of offerings in compliance with these criteria increased from $33 \%$ to $82 \%$, demonstrating successful implementation of the guidelines.

\section{Facilitators of Implementation}

Whatley Blum et al. (2007) evaluated HFEP implementation among four high schools implementing low-fat low-sodium requirements. The authors identified that 
technical assistance and education of school food service personnel were key factors in the successful implementation of HFEPs. Further, the level of education for personnel correlated with compliance. Bamford and colleagues (2012) echoed these findings among implementation efforts among long-term care facilities, underscoring technical assistance and institutional support as critical elements for staff buy-in. Another study reported that including registered dieticians in implementation efforts fostered collaborative efforts that informed key decision makers (Brown et al., 2009). Similarly, Masse et al. (2013) concluded that the availability of a nutritionist for consultation was a facilitator of implementation within school settings.

Gase and colleagues (2011) identified three facilitators among nine county government departments implementing healthy procurement policies: formal prioritization of nutritious foods, legal authority to mandate practices, and existing nutrition policies (i.e., incrementalism). Another study of county government units in Los Angeles reported facilitators of HFEP implementation as high-quality technical assistance, education of "end-users" (e.g., consumer-facing staff, consumers), and social media marketing campaigns (Robles et al., 2013). The authors noted that the implementation setting (county government) may have afforded decision-makers (county officials) greater bargaining authority with food retailers than could have been possible in other settings.

\section{Barriers to Implementation}

In 2009, Anderson et al. conducted a systematic review of HFEPs in the workplace, including barriers to implementation, however the authors found no barriers 
reported. Robles and colleagues (2013) studied HFEPs within county government and cited budget constraints, consumer resistance, and lack of adequate staffing as barriers to successful implementation of HFEPs. Gase and colleagues (2011), in discussing barriers to implementing a low-sodium nutrition policy among nine county departments, identified the following: (1) resistance among departments to a "one-size-fits-all" policy; (2) higher cost of low-sodium items; (3) complexity of service arrangements among departments; (4) consumer resistance of changes to offerings; and (5) lack of knowledge in operationalizing policy as barriers to implementation. However, the authors acknowledge the role of the setting (county government) as limiting the generalizability of barriers to other sites.

In a hospital-based study, Bell et al. (2013) reported that hospital administrators were loath to remove competitive ultra-processed foods due to fear of revenue loss. School administrators expressed the same concern, citing constrained budgets and conflict with "competitive foods" purveyors (Cohen et al., 2016; Wharton, Long \& Schwartz, 2008). In a review of government policy barriers for HFEP adoption, Mozaffarian and colleagues (2018) reported that limited budgets and perceived fear of revenue loss were obstacles. Similarly, an evaluation of a city-wide executive order for healthy beverages reported losses in profit as a barrier for full implementation (Cradock et al., 2015).

In examining public-private partnership efforts, Park and Lee (2016) conducted focused in-depth interviews with food catering companies and identified that sales, not nutritional quality, were the first priority for worksite cafeterias. This finding was also 
reported in a study by Olstad and colleagues (2013) examining the tension between nutritional guidelines and corporate profitability. Likewise, Vander Wekken and colleagues (2012) found that negative impact on profits was a top concern of industry partners implementing HFEPs within publicly-funded retail venues. This review of the literature underscored that the persistent fear of revenue loss across public and private sectors was as a recurrent barrier to implementation for HFEPs.

\section{The Role of Health Care Institutions in Addressing the Obesity Epidemic}

Lesser and Lucan (2013), in their assessment of a hospital's role in modeling healthy contexts, argued (p. 300):

Given that many leading causes of preventable illness and premature death in the U.S. - obesity, diabetes, heart disease, cancer- are diet-related, it is logical that hospitals have a stake in providing health-promoting food. Doing so helps a hospital fulfill its mission to prevent disease and promote wellness and health, both by sending a message about proper nutrition and by nourishing patients, students, volunteers, staff, visitors, and others... However, if a hospital cafeteria achieves profitability by selling items that promote poor eating habits and poor health, there is a conflict between that business practice and the hospital's broader mission. Certainly, a hospital might generate valuable revenue by selling any number of products that are bad for one's health (e.g., cigarettes). But selling such products would contradict the health-driven mission, and any revenue generated would not be a defensible offset. Offsets from selling foods that clearly damage human health would, likewise, be indefensible.

The authors' position was squarely rooted in the institutional position that hospitals

cannot contradict their health-driven mission for the sake of financial profit. Whereas a manufacturing plant or an advertising agency may not have any explicit obligation to promote health, health care organizations have an ethical and professional responsibility to abstain from the promotion of destructive or unhealthy products and practices. From an 
institutional perspective, health care organizations have employed diverse strategies in addressing the obesity epidemic, as mentioned briefly in Chapter 1. Examples included hospital systems evolving best practices within allopathic obesity care, state Medicaid programs covering pharmacologic therapies for overweight and obesity, and health insurers expanding coverage of obesity treatment modalities. Health systems also coordinated with governmental and community organizations to facilitate practice- and evidence-based research studies to refine the knowledge base on obesity prevention initiatives (Huang \& Glass, 2008).

Health organizations have also applied a range of interventions to address the threat of obesity for its constituents: many large health care organizations, such as hospital systems, have sponsored wellness programs to lower morbidity and mortality associated with obesity (Kaspin, Gorman \& Miller, 2013). Examples of wellness program components targeting obesity included nutrition classes, health risk appraisals, onsite exercise facilities and access to dieticians (Estabrook, Zapka \& Lemon, 2012). Though health care organizations are not alone in this practice, organizations within the health field possessed a particular advantage in their ability to offer preventive services given their infrastructure and setting, such as the ability to conduct health risk appraisals internally and provide onsite access to medical staff (Heinen \& Darling, 2009). Perhaps the most obvious wellness program component to address obesity has been the provision of employer-sponsored weight management programs, though these programs were more often offered by larger organizations (53\% of large organizations offered weight management programs compared to $16 \%$ of small organizations) (Gabel et al., 2009). 
Another group of institutional health care actors responding to the obesity epidemic has been state Medicaid programs. As the evidence base has expanded regarding obesity treatments, nearly all state Medicaid programs cover and reimburse a combination of therapies and procedures, including intensive behavioral therapy, medical nutrition therapy, nutritional counseling, dietician visits and bariatric surgery (Jannah et al., 2018; STOP, 2017). A number of states also cover commercial weight management programs such as Jenny Craig and Weight Watchers (HMO, 2007). While states varied in which services were covered, the growing number of programs that offered obesity prevention and treatment services signaled the status change of obesity as a legitimate disease. To date, there are over 15 ICD-10 codes used for billing adult weight management services by Medicaid (Ethicon, 2018).

A growing number of state health agencies have also identified obesity among the priority areas for improving public health (OHA, 2015; Pomeranz, 2011; Slater et al., 2007) — state efforts include increasing the price of sugary drinks, building capacity among communities to employ obesity prevention initiatives, and creating incentives for public and private health plans to engage in obesity prevention (OHA, 2015; OPP, 2018). Finally, a growing number of hospital systems have instituted comprehensive obesity programs, particularly within children's hospitals (Eneli et al., 2011).

\section{New Institutionalism}

Health care institutions promote health and well-being through the organization and delivery of health services. These organizations shape perceptions about healthy 
behaviors and act as authoritative figures on guiding policy and practice regarding all aspects of health. As public health crises mount and subside, society looks to these institutions for guidance and best practices. The role of health care institutions in modeling healthy contexts through food environments is rooted in institutional theory. According to Cairney and Heikkila (2014, p. 364), institutions are comprised of:

Rules, norms, practices and relationships that influence individual and collective behavior... Institutions at one level (e.g., constitutional) can also shape activity at another (e.g., legislative or regulatory), establish the types of venue where policy decisions are made, and make the rules that allow particular types of actors to enter.

Health care's institutional role in society is legitimized in its expertise and knowledge of health, health promotion and delivery of health services. As the obesity epidemic grows larger with each decade, institutional actors have acknowledged the multifactorial nature of obesity and the multidisciplinary approach needed to address such chronic diseases. The ACA has improved opportunities for screening obesity as well as including obesity monitoring as a process quality measure for many alternative payment models (Parekh, 2017). Whether the US health care system will invest more heavily in institutional actors and employer-sponsored programs to address obesity or transition to universal models of care remains unknown (Gabel et al., 2009). Notwithstanding, the management of obesity is not well-suited within the current traditional fee-for-service model, which rewards quantity over quality and is an unsustainable payment mechanism for chronic disease management (Hussey et al., 2014). Updating models of care and provider reimbursements, such as paying for obesity treatments via bundled or comprehensive perpatient payments as opposed to clinician-directed per visit fees, are emerging as potential 
solutions (Chen \& Bodenheimer, 2008). Ensuing discussions about health care system redesign acknowledge the need to evolve pay-for-performance mechanisms (NASEM, 2017).

Poor food environments are ubiquitous (Hall, 2018). As Figure 2.1 illustrated above, the ways obesity is influenced across multiple socioenvironmental levels, especially within the community-, agricultural- and industry-levels, underscores that most food environments are obesogenic (Swinburn et al., 2015). While this may be relevant to every venue within society, poor food environments within hospitals are especially visible and draw harsh criticism (Cram et al., 2002; Wojcicki, 2013). The institutional mission of these organization is health promotion and delivery — as noted in Chapter 1, hospitals and health agencies are expected to set an example of healthy behaviors and provide healthy contexts.

\section{Applying HFEPs: The Role of Organizational Context}

Organizational environments are not given realities; they are created through a process of attention and interpretation (Weick, 1988). Though the grey and refereed literature is replete with toolkits and tips for success regarding how to change food environments, few interventions are discussed in-situ- further, if an intervention is not successful, explanations often conclude the intervention was not implemented "correctly," as opposed to looking at the situational environment and speculating on the fit (Plsek, 2003). In this section, a discussion clarifying the concept of organizational context is presented in order to understand the relationship between an intervention and 
its setting. The definition of organizational context for this dissertation is discussed as well as definitions for each of the key elements of this construct. Finally, the degree to which organizational context may influence implementation success is reviewed, justifying the selection of the conceptual framework, which is borrowed from implementation science.

\section{Organizational Context: Conceptual and Operational Definitions}

Organizational theorists have historically emphasized the need to adapt to environments to remain viable - external threats often shape organizational decisionmaking as well as facilitate processes by which organizations achieve their goals (Hannan \& Freeman, 1974; Aldrich \& Pfeffer, 1976). Facilitating this process often requires the formation of strategic and operational goals in order to cope with uncertainty (Thompson, 1967). The characteristics of how, why, and the degree to which organizational contexts affect organizational strategy and success is key to examining how organizations minimize uncertainty by creating coping mechanisms (e.g., standard operating procedures) and reducing ambiguity (Lawrence \& Lorsch, 1967). Uncertainty consists of three components: (1) the lack of clarity of information, to which Jones (2003) states that when the goals for an organization are hazy, this lack of clarity reverberates throughout subsequent processes and strategies; (2) delays in feedback amplifies the presence or absence of feedback loops, often creating vulnerabilities within organizations to swiftly react to threats; (3) general uncertainty regarding causal relationships leads to unclear “pulls and pushes" (Hannan \& Freeman, 1993). 


\section{Definition for this Study}

As this dissertation sought to understand how organizational factors affected the implementation of HFEPs, studying the organizational characteristics and context of different health care organizations as well as observing how HFEPs are adopted and implemented inform how context plays a role in shaping implementation challenges and how organizational characteristics (e.g., structure, resource availability) create environments in which a factor can be a barrier in one context or a facilitator in another. As mentioned in Chapter 1, the organizational characteristics of interest to this study included: structure (governance, size, interorganizational coupling), receptivity (tension for change, norms and culture), resource availability (slack resources, social network location, information sharing), and leadership (change agents, stakeholders, vision).

\section{Structure: Governance, Size, Interorganizational Coupling}

Governance Governance implies hierarchy and control; thus, studying governance structures among organizations is critical to understanding the behavior of organizations within networks (Williamson, 1991). Provan and Kenis (2008) define governance as "the use of institutions and structures of authority and collaboration to allocate resources and to coordinate and control joint action across the [organization] as a whole" (p. 231). This definition expands upon earlier work characterizing governance as having unique structures, modes of conflict resolution and bases for legitimacy (Jones et al., 1998). Governance shapes the degree to which organizations are hierarchical and formal or 
decentralized and casual (Foss, Husted, Michailova \& Pedersen, 2003). Decision-making processes and strategy formation flow directly from governance structure in a top-down fashion (Hill \& Lynn, 2004). The organization's strategy subsequently determines the tasks and technology of the organization, which, in turn, feeds back into the governance structure, underscoring the cyclical and interconnectedness of this factor in forming and adapting strategy. Similar governance structures are predictive of collaboration and fostering kinship between organizations (Gulati, 1999). Despite widespread study of governance structures in organizational literature, little attention has been paid to how governance structures of interorganizational networks, as opposed to a single organization, can shape strategy (Salancik, 1995). Broadening the unit of analysis to whole systems can inform levers for scaling interventions. In studying governance in this dissertation, the degree of hierarchy and centralization will be studied to determine if it is a facilitator or barrier to decision-making regarding food environments. The governance structures of organizations can typically be modified through perturbations in technology, information, and resources (March \& Olsen, 1983).

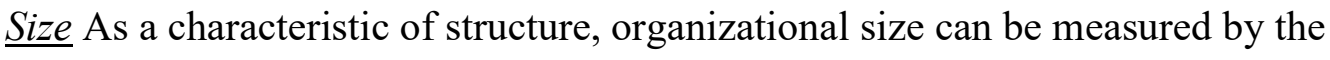
number of employees, the annual operating budget or the size of assets of the organization (Aiken \& Hage, 1971). Alternatively, size can be measured as a relative construct: from input or output volume to personnel capacity between groups. The larger an organization, the more resources are typically available: marketing skills, administrative capacity and human capital are all examples of such resources (Daft \& Becker, 1980). However, small organizations possess more flexibility, thereby being 
more adaptive to challenges and receptive to innovative solutions. Thus, the size of an organization may not uniformly predict whether innovative practices are encouraged or inhibited. In a meta-analytic review of the relationship between an organization's size and innovation, Damanpour (1992) reviewed twenty studies and found a positive relationship. However, the positive correlation was more likely observed within manufacturing firms as compared to non-profit organizations, suggesting that size alone does not determine an organization's inclination to experiment with innovation. Further, the author concluded that the size of an organization more strongly related to implementation success than to the adoption decision — suggesting that size may be both a facilitator (in implementation) while simultaneously acting as a barrier (in adoption).

Mohr (1969), in an attempt to construct a standardized measure for innovativeness, found no relationship with organizational size. However, the author found a positive relationship between expenditures and innovative behavior, suggesting that higher incomes could imply greater discretion. Damanpour (1992) suggested that the stage of adoption can better predict whether size plays a role, as the nature of activities between stages of adoption vary widely: "the initiation stage depends on individuals to perceive the problem, gather information and form an attitude... while the implementation stage depends on chang[ing] systems, structures, and behaviors" (p. 379). Thus, the size of an organization may indicate its propensity to facilitate change as opposed to motivating innovativeness.

Interorganizational Coupling Originally labeled "inter-organizational analysis," the study of networks of organizations sought to understand the dependent nature of 
organizations on one another (Evan, 1965). Both social dynamics in networks and open systems theory underscore how the interdependence of organizations shapes neighboring coalitions, cements interdependencies, and couples organizations as each organization relies on the resource terrain of its surroundings (Katz \& Kahn, 1978). Determining the role of interorganizational reliance in various stages of search, selection and implementation of HFEPs was of interest in this study. On a spectrum of loose-to-tight coupling (Weick, 1976), opportunities for stakeholder collaboration can result in both shared resources as well as the risk of shared losses. Additionally, the propensity to cooperate or compete (Tjosvold, 1998) can be inferred based on the heterogeneity of the organizations in a network (Gulati, 1999).

The opportunities involved for interorganizational cooperation are particularly highlighted in this factor as heterogeneity of values can serve complementary roles in symbiotic relationships. However, organizational cultures and corresponding compatibilities can rarely be predicted and often are a source of conflict between organizations. Observed value differences among heterogeneous organizations may initially disincline organizations to cooperate with diverse organizations, instead choosing to insulate further from uncertainty and rely on organizations with similar values to sustain resources and obtain goals. However, if organizations are sufficiently dissimilar, competition for similar resources may be minimal; thus, cooperation may be an acutely rational response to mitigating uncertainty. Irrespective of the ultimate choice to cooperate or compete with organizations within a network, heterogeneity is a factor that may also explain inter-organizational behavior: acknowledging the uncertainty that 
heterogeneity produces may be a valuable construct in both explaining and predicting behaviors among organizations within networks.

\section{Receptivity: Tension for Change and Norms and Culture}

Tension for Change Beer \& Nohria (2000) describe tension as an archetype: the path of least resistance is always preferred. Organizational tension, much like psychological tension in people, is more often avoided and ignored than proactively dealt with (Judge \& Blocker, 2008). However, as external pressures, such as the obesity epidemic, continue to mount and resource landscapes change, tension for solutions rise and the presence, or absence, of this factor on receptivity can determine whether organizational actors are amenable to change (Zajac \& Shortell, 1989; Ghemawat \& Costa, 1993). Further, differentiating between internal pressures (change agents) and external pressures (public criticism) can elucidate the nature of this organizational factor in determining the implementation landscape.

Norms and Culture The professional values and norms of an organization are important factors in strategic decision-making (Tosti \& Jackson, 2000). Culture is described as "how" things get done - with values and norms guiding behaviors and practices (Zheng, Yang, \& McLean, 2010). The influence of organizational culture on receptivity for change and improvement is high (Ott, 1989). Given the organizations of interest for this study were embedded with a highly professional and institutional context, exploring the relationship between public health concern and profit-seeking motivations was helpful in determining this factor's importance in shaping organizational context. 
Norms and culture influence the scope of actions considered, the degree to which organizations adapt to changing information, and the manner in which health care organizations respond to challenges.

Resource Availability: Slack, Social Network Location and Information Sharing

Resources can take the shape of monetary funds, attention, bargaining power, access to stakeholders, as well as many other forms pertinent to organizations (Meyer \& Scott, 1992). Given that highly finite and scarce resources exist in the public and government realms (as opposed to private), network of organizations must compete with contenders, including other disease networks, for fixed funds and limited attention (Kenis \& Schneider, 1991). Katz and Kahn (1978), within their evolutionary perspective, have purported that organizational environments "select" organizations for survival, providing and withholding resources. Carroll and Hannan (2003) closely examine how organizations exchange resources within environments as a condition for survival-the dependency of organizations on their environments exhibits how vulnerable organizations may or may not be to system perturbations. Whether resources take shape in information, funds, attention, or nodal placement within networks (Levine \& White, 1961), such elements are emblematic of the environments' inextricable relationship with organizations.

Slack Resources Originally coined by Cyert \& March (1963), organizational slack is defined as resources "in excess of the minimum necessary" to produce an acceptable level of organizational output. These resources represent a flexible cushion for 
organizations to experiment with novel technologies, implement new models of care, adjust to internal or external environments, and course correct organizational strategy. Penrose (1959) provided the foundation for this phenomenon by describing excess resources as "important determinants of organizational structure, growth and performance" (p. 483). Bourgeois (1981) later added to Cyert \& March's definition of slack as a "resource cushion" that organizations can utilize in order to fend off threats as well as exploit time-sensitive opportunities.

Slack resources have since been studied and further refined, with three typologies emerging: available, recoverable and potential slack resources. Available slack can be typically regarded as monetary or otherwise liquid resources — not yet invested into the organization and thus highly valuable (Singh, 1986). Cash flow, net sales, and quick ratio are all examples of available slack resources. Recoverable slack is considered as those resources that have already been invested into an organization or activity but can be otherwise repurposed — a unit absorbing additional responsibilities, a consolidation of tasks among organizational units, or recovered overhead or inventory costs are all examples of this typology. Finally, potential slack resources can most easily be exemplified as raising organizational debt load: resources that may become available through a hospital's activity in soliciting resources from the external environment. Health care organizations typically possess all three typologies of slack resources.

However, these resources are not uniformly regarded positively: a divergent perspective cites the existence of slack resources as evidence of too much "fat" in the health care system (Zinn \& Flood, 2009), wherein slack resources are considered as 
inappropriate and wasteful elements of an inefficient system. Anchored in a neoclassical perspective, the objective of any firm is to operate at optimal efficiency in order to maximize profits (Meyer, 1982). Slack resources can be an indication that optimal efficiency has not been reached. Hollingsworth (2008), in a review of US hospital efficiency, reported a consistent pattern of organizational maintenance of slack resources, representing a striking divide between theoretical commitments and actual behavior among hospitals. However, this review concluded that availability of slack resources permitted hospitals to adjust to external challenges and explore new technologies. Thus, slack resources are a critical component of an organization's ability to innovate (Cyert \& March, 1963). The availability of a "cushion" is necessary for an organization to be able to experiment with new ways of responding to challenges and trial-run novel strategies to said challenges.

Social Network Location Also known as cosmopolitanism, organizations' access to resources and information is determined by their social network location (Robertson \& Wind, 1983). Organizations within networks are differentially placed given their specification - how diffuse or concentrated an organization's location is determines their value, power, and access to tangible resources (Katz \& Kahn, 1978). The location of organizations within networks also determines the kinds of organizational benefits that are conferred due to its placement. The placement (e.g., node) of an organization within an interorganizational community can be a benefit (stability) or a weakness (vulnerability), in that shocks experienced by neighboring organizations may reverberate throughout the network and nodes, destabilizing the system. The benefits of stability 
typically have the concomitant disadvantage of rigidity (Frickel \& Moore, 2006). Further, the impact of technological breakthroughs on organizations (Utterback, 1974) can either debilitate or solidify entire networks (Tushman \& Anderson, 1986). Opportunities for collaboration among similarly situated organizations, such as the Healthy Food Financing Initiative, can result in both shared resources as well as the risk of the degree to which shocks in one organization will be felt by neighboring nodes.

Developed from Katz and Kahn's (1978) “ecological perspective,” technology symbolizes an evolutionary innovation that enables organizations to adapt to changing landscapes and remain "fit for survival" (Lewin, Long \& Carroll, 1999). Population ecology stresses the ability to adapt in order to anticipate and weather uncertainty (Nelson, Adger \& Brown, 2007); thus, organic organizations are poised to overcome exogenous shocks in comparison to bureaucratic, mechanistic organizations that value stability but may be hindered by rigidity.

Information Sharing Tightly knit micro-networks promote reliable communication (Lasker et al., 2001; March \& Simon, 1993; Baker, 2002). The coupling of organizations through open channels of communication directly affects the degree to which goals may become aligned and collaboration fostered. Open communication is characteristically essential for organizational effectiveness (Lasker et al., 2001). Information technology permits organizations to adopt novel behaviors in adaptation to shifting dynamics within networks. Among closely coupled health organizations, identifying the facilitators and barriers to communication among and between organizations becomes essential. Uni-directional or costly communication are two 
barriers to achieving synergy and cooperation (Alter \& Hage, 1993). Given the availability and accessibility of two-way communication between organizations, this factor can be instrumental in efficient and effective communication. Conversely, lack of bi-directional communication can pose as a barrier.

\section{Leadership: Change Agents, Stakeholders and Vision}

Change Agents Though size and organizational culture shape strategic trajectories of health care organizations, the presence of champions and change agents is strongly linked to initiating and facilitating implementation of innovations (Cohen et al., 2016; Wharton, Long \& Schwartz, 2008). Fairholm (2009) describes the critical role of leaders as agenda-setters: determining objectives, setting priorities and anticipating potential opportunities.

$\underline{\text { Stakeholders }}$ Customers, shareholders, suppliers, employees, and the general public are examples of stakeholders for any given organization. Each stakeholder group may have their own values and priorities, thus the need to appeal to all relevant decisionmakers highlights the role of this characteristic in shaping organizational context. In a sample of 148 firms, Brammer \& Millington (2003) analyze the relationship between stakeholder attitudes, community involvement and organizational structure, concluding that the industry that the organization is nested within greatly shapes corporate social responsibility (CSR) activities. CSR has emerged as a significant pressure on contemporary organizations (Kapstein, 2001), with larger firms allocating separate 
resources for CSR activities while smaller organizations achieve community involvement goals through central administrative functions (Brammer \& Millington, 2003).

Fernandez and Rainey (2017) report that stakeholder and top-management support is critical in successfully innovating change within public service organizations, identifying "all types" of stakeholders: frontline staff, union delegates, managers, as well as governmental figures if applicable. Ramus and Vaccaro (2017) describe the tension between stakeholders when social enterprise/CSR activity poses a threat to the profitseeking activities of the organization, described as "mission drift." This drift can widen differences among stakeholders and often leads to a decrease in CSR activity. Smith et al. (2013), in describing ways to prevent this phenomenon, suggests that maintaining visible social commitment in the community as well as open communication between stakeholders can be helpful in ensuring CSR commitment. Thus, reaching consensus among stakeholders on organizational strategy is necessary for successful implementation of innovations.

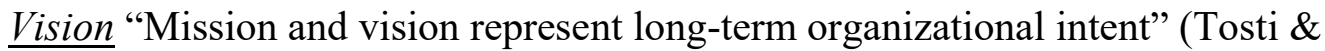
Jackson, 2000, p. 2). Strategic decision-making takes the missional values of an organization and organizes resources to realize those values through action (Foster \& Akdere, 2007). Tosti and Jackson (2000), in a review of organizations as systems, describe the vision of an organization as the guiding principle for both organizational culture as well as strategy. The authors emphasize that any innovations to a system must align in order to have long-term compatibility. 


\section{Organizational Context and Implementation Success}

Whereas innovation and diffusion research efforts attempt to broadly appeal to a wide audience, organizational context represents a need for custom-tailored solutions and case-by-case considerations (Glisson \& Schoenwald, 2005; Plsek, 2003). From the review of organizational characteristics, the absence or presence of certain factors result in distinct opportunities or challenges to changing the food environment. For example, the size of an organization or its degree of centralization may be a barrier in garnering enough buy-in for the decision to adopt HFEPs; conversely, a well-connected health care system can learn from associated systems and sidestep known implementation challenges. Additionally, the perceived and actual costs of implementing HFEPs may differ based on an organization's information channels or organizational culture. Perceived costs may under- or over-estimate the actual effort needed to implement this intervention. The degree to which these factors play a real role in decision-making and implementation vary in influence (Birken et al., 2015).

Perceived costs, also known as transaction costs, can play a significant role in determining organizational strategy (Williamson, 1989). Further, transaction costs differ among organization given resources and constraints, meaning that both resource commitments as well as implementation processes will differ from one organization to the next. In their systematic review of diffusion of innovations within organizations, Greenhalgh et al. (2004) caution that innovations whose perceived benefits are outweighed by perceived costs are unlikely to be implemented, regardless of the actual costs and benefits. Plsek (2003), in discussing innovation spread among complex health 
care systems, cautions that organizational context is an often-overlooked element. Porter, Allen \& Angle (2003) submit that organizations strive to implement solutions that have the greatest (perceived) benefit for the lowest cost. However, the "costs of search," coupled with environmental uncertainty and ambiguous external pressures, create a terrain that requires significant upstart resources in order to commit to a strategy (Lubell et al., 2017; Birken et al., 2015).

In order to study organizational context and its relationship to implementation success, an adapted model of Rogers' (2003) Five Stages in the Innovation-Decision Process served as the conceptual framework to guide this study. The decision to use a well-cited framework from the field of implementation science was intentional in order to adequately capture the process of application. This model's emphasis on context aligned with the study's goal of examining diverse organizational structures of health care systems. As HFEPs continue to grow in popularity and uptake, the extent of organizational coupling in determining dissemination strategies or the innovation-system fitness as a predictor of implementation success were two queries this study sought to address. As summarized by May and colleagues (2016), internal organizational conditions are becoming increasingly reflected in implementation frameworks. This work of studying organizational context and its influence on implementation success adds to this literature.

\section{Summary}

This chapter sought to review the relevant literature for this dissertation. An overview of the obesity epidemic, including framing, drivers and prevention strategies set 
the backdrop for the dissertation study. Population-level obesity prevention strategies are necessary for meaningful change, and food environments are both largely responsible for this epidemic as well as pivotal settings within which population-level strategies can be implemented. HFEPs are a tool that can structurally change these environments, and the application and evaluation of HFEPs within retail health care environments is sparse.

Additionally, a review of the innovation-system fitness suggests that applying these practices within health care environments is particularly robust. Health care institutions play both a pragmatic and symbolic role in society, whereupon sanctioned behaviors, expertise and practices are shaped by professional norms and values. Health care organizations are expected to promote and deliver health, and the added expectation of accomplishing these aims while modeling healthy behaviors is particular to this field. However, notwithstanding societal expectations and public sanction, health care organizations are organizations that are boundedly rational, experience uncertainty, have limited information, and are pressed to act timely despite resource limitations and unclear strategy. Further, the obesity epidemic, similar to other national health emergencies, is complex in scope and no clear solution exists. Thus, such institutions look to neighboring organizations to shape strategy, either through mimetic, normative or coercive isomorphism. The growing popularity of HFEPs as a strategy for modeling healthy contexts by a handful of early adopters has signaled to other health care organizations that this strategy is legitimate and worthwhile. However, the application and evaluation of this strategy, as reviewed, is limited. Further, the role of the organizational context in shaping the facilitators and barriers of effective application is another gap in the literature 
that this dissertation addressed. The review of organizational elements that comprised this context suggested that any combination of organizational factors may shape distinct challenges to application of a given intervention. Thus, studying organizational context was a worthy endeavor to further application efforts and inform dissemination strategies. 


\section{CHAPTER 3: METHODOLOGY}

This chapter presents a detailed overview of the mixed methods study exploring HFEP implementation within health care organizations. This work utilized qualitative methods to describe facilitators of and barriers to HFEP implementation within six health care organizations (Aim 1), quantitative methods to assess the economic and consumer behavior impacts of one health system's region-wide sugar-sweetened beverage ban (Aim 2), and qualitative methods to determine the organizational contexts of participating institutions and describe the relationship between contexts and HFEP selection (Aim 3).

\section{Operationalization of Key Concepts}

This research sought to answer the following question: How does the organizational context of health care organizations affect the implementation process and economic outcomes of healthy food environment policies? Table 3.1 operationalizes each concept of the key research question. Further, Rogers' (2003) adapted model of Five Stages in the Innovation-Decision Process provided the conceptual framework that guided the research study. This adapted model addressed the main elements of the study and conceptualized how the environmental context (e.g., the obesity epidemic and subsequent institutional responses) and organizational context (structure, resources, receptivity and leadership) were inputs in determining the adoption decision to implement HFEPs. These input factors also affected the process of implementation in that the broader environment as well as organizational context shaped the kinds of factors that facilitated or inhibited implementation of HFEPs. Outcomes, namely changes in revenue 
and consumer behavior, were a direct result of the implementation process, as described

in Figure 1.1 in Chapter 1.

\section{Table 3.1 Operationalization of Key Concepts}

\begin{tabular}{|c|c|c|}
\hline Concept & Operational Definition & Source \\
\hline $\begin{array}{l}\text { Health care } \\
\text { organization }\end{array}$ & $\begin{array}{l}\text { Any consumer-facing organization that is within the health } \\
\text { care industry, including hospitals, clinics, medical offices, } \\
\text { and public health agencies. Other health care organizations } \\
\text { such as health insurance companies, hospice homes and } \\
\text { education centers are excluded. }\end{array}$ & $\begin{array}{l}\text { Institute of } \\
\text { Medicine, } \\
2003\end{array}$ \\
\hline HFEPS & $\begin{array}{l}\text { Any combination of the following policies instituted within } \\
\text { the retail environment: (1) trans-fat and/or sodium limits; } \\
\text { (2) limiting or banning access to sugar-sweetened } \\
\text { beverages; (3) instituting serving size limits; (4) prioritizing } \\
\text { healthy methods of food preparation (e.g. removing fryers); } \\
\text { (5) nutrition labelling for meals and items at point of } \\
\text { service; (6) promotion of healthy foods and beverages in } \\
\text { marketing materials; (7) using pricing strategies to increase } \\
\text { the affordability of healthy items; (8) healthy vending } \\
\text { policies; and (9) increasing access to healthy foods and } \\
\text { beverages through other means. }\end{array}$ & $\begin{array}{l}\text { Review of } \\
\text { grey and } \\
\text { refereed } \\
\text { literature } \\
\text { (Chapter 2) }\end{array}$ \\
\hline $\begin{array}{l}\text { Organizational } \\
\text { context }\end{array}$ & $\begin{array}{l}\text { Characteristics including structure (governance, size, } \\
\text { interorganizational coupling), receptivity (tension for } \\
\text { change, norms and culture), resource availability (slack } \\
\text { resources, social network location, information sharing), and } \\
\text { leadership (change agents, stakeholders, vision). }\end{array}$ & $\begin{array}{l}\text { Miller, } \\
\text { Droge \& } \\
\text { Toulouse, } \\
1988\end{array}$ \\
\hline $\begin{array}{l}\text { Environmental } \\
\text { context }\end{array}$ & $\begin{array}{l}\text { Felt needs; professional norms; public opinion; norms of the } \\
\text { social system; broader systems that shape external threats } \\
\text { and institutional pressures. }\end{array}$ & $\begin{array}{l}\text { Rogers, } \\
1983 ; 2003 .\end{array}$ \\
\hline $\begin{array}{l}\text { Adoption } \\
\text { decision }\end{array}$ & Steps and/or decisions made to implement HFEPs. & $\begin{array}{l}\text { Rogers, } \\
1983 ; 2003 .\end{array}$ \\
\hline $\begin{array}{l}\text { Implementation } \\
\text { Process }\end{array}$ & $\begin{array}{l}\text { Any deliberately initiated attempt to introduce new, or to } \\
\text { modify existing, patterns of action in health care or some } \\
\text { other formal organizational setting. Deliberate initiation } \\
\text { means that an intervention is: institutionally sanctioned; } \\
\text { formally defined; consciously planned; and intended to lead } \\
\text { to a changed outcome. }\end{array}$ & $\begin{array}{l}\text { May et al., } \\
2007\end{array}$ \\
\hline $\begin{array}{l}\text { Economic } \\
\text { Outcomes }\end{array}$ & $\begin{array}{l}\text { Short- and medium-term revenue effects; changes in } \\
\text { consumer purchasing behavior. }\end{array}$ & $\begin{array}{l}\text { Grech et al., } \\
2015\end{array}$ \\
\hline
\end{tabular}




\section{Organizational Context}

A number of organizational characteristics were of interest to the study, including structure (governance, size, interorganizational coupling), receptivity (tension for change, norms and culture), resource availability (slack resources, social network location, information sharing), and leadership (change agents, stakeholders, vision). Examining how these characteristics did or did not present barriers to effective implementation could inform prospective organizational decision-makers of environments in which a factor can be a barrier in one context or a facilitator in another. Table 3.2 operationalizes each domain (and respective conceptual variables) of organizational context.

Table 3.2 Operationalization of Organizational Context

\begin{tabular}{|c|l|l|l|}
\hline Domain & \multicolumn{1}{|c|}{ Variable } & \multicolumn{1}{c|}{ Operational Definition } & \multicolumn{1}{c|}{ Source } \\
\hline \multirow{5}{*}{ Structure } & Governance & $\begin{array}{l}\text { The use of institutional and structural } \\
\text { authority to collaborate, allocate } \\
\text { resources, resolve conflict, and control } \\
\text { an organization through decision-making } \\
\text { and strategy formation. }\end{array}$ & $\begin{array}{l}\text { Williamson, 1991; } \\
\text { Jones et al., 1998; } \\
\text { Hill \& Lynn, 2004; } \\
\text { Provan \& Kenis, } \\
\text { 2008 }\end{array}$ \\
\cline { 2 - 5 } & Size & $\begin{array}{l}\text { The number of employees within an } \\
\text { organization as well as operating budget. }\end{array}$ & $\begin{array}{l}\text { Aiken \& Hage, } \\
1971 ; \text { Damanpour, } \\
1992\end{array}$ \\
\cline { 2 - 5 } & $\begin{array}{l}\text { Inter- } \\
\text { organizational } \\
\text { coupling }\end{array}$ & $\begin{array}{l}\text { The degree to which an organization is } \\
\text { dependent on others within a network in } \\
\text { order to achieve goals; the degree to } \\
\text { which organizational processes, } \\
\text { resources, and decision-making are } \\
\text { shared. }\end{array}$ & $\begin{array}{l}\text { Evan, 1965; } \\
\text { Weick, 1976 }\end{array}$ \\
\hline \multirow{5}{*}{ Receptivity } \\
\cline { 2 - 5 } & $\begin{array}{l}\text { Tension for } \\
\text { change }\end{array}$ & $\begin{array}{l}\text { The degree to which external pressures } \\
\text { mount within an organization; internal } \\
\text { processes are discordant with needs of } \\
\text { the organization; and imperative for } \\
\text { organizational action is heightened. }\end{array}$ & $\begin{array}{l}\text { Zajac \& Shortell, } \\
1989 ; \text { Beer \& } \\
\text { Nohria, 2004 }\end{array}$ \\
\cline { 2 - 5 } & $\begin{array}{l}\text { Norms and } \\
\text { culture }\end{array}$ & $\begin{array}{l}\text { The professional values of an } \\
\text { organization that guide behaviors and } \\
\text { practices; influence of organizational } \\
\text { climate on strategic decision-making. }\end{array}$ & $\begin{array}{l}\text { Ott, 1989; Tosti \& } \\
\text { Jackson, 2000 }\end{array}$ \\
\hline
\end{tabular}




\begin{tabular}{|c|c|c|c|}
\hline \multirow{3}{*}{$\begin{array}{c}\text { Resource } \\
\text { Availability }\end{array}$} & $\begin{array}{l}\text { Slack } \\
\text { resources }\end{array}$ & $\begin{array}{l}\text { Maintenance of excess organizational } \\
\text { resources in order to experiment with } \\
\text { novel technologies, course-correct } \\
\text { organizational strategy, and adjust to } \\
\text { internal and external needs. Typified into } \\
\text { liquid, potential and recoverable slack. }\end{array}$ & $\begin{array}{l}\text { Cyert \& March, } \\
\text { 1963; Bourgeois, } \\
\text { 1981; Meyer, 1982; } \\
\text { Singh, 1986; } \\
\text { Hollingsworth, } \\
2008\end{array}$ \\
\hline & $\begin{array}{l}\text { Social } \\
\text { network } \\
\text { location }\end{array}$ & $\begin{array}{l}\text { The "placement" of an organization } \\
\text { within an interorganizational community } \\
\text { as it relates to the degree of distance to } \\
\text { tangible resources and power; a "node" } \\
\text { within a network of coalitions, } \\
\text { information and technology. }\end{array}$ & $\begin{array}{l}\text { Katz \& Kahn, } \\
\text { 1978; Tushman \& } \\
\text { Anderson, 1986; } \\
\text { Nelson, Adger \& } \\
\text { Brown, } 2007\end{array}$ \\
\hline & $\begin{array}{l}\text { Information- } \\
\text { sharing }\end{array}$ & $\begin{array}{l}\text { The presence or absence of reliable, } \\
\text { open channels of communication within } \\
\text { and between organizations for } \\
\text { maximized effectiveness, collaboration } \\
\text { and goal alignment. }\end{array}$ & $\begin{array}{l}\text { March \& Simon, } \\
\text { 1993; Alter \& } \\
\text { Hage, 1993; Lasker } \\
\text { et al., 2001; Baker, } \\
2002\end{array}$ \\
\hline \multirow[t]{3}{*}{ Leadership } & $\begin{array}{l}\text { Change } \\
\text { agents }\end{array}$ & $\begin{array}{l}\text { Executive-level or management } \\
\text { "champion" that initiates, prioritizes and } \\
\text { facilitates organizational innovation; } \\
\text { agenda-setters that determine objectives } \\
\text { and anticipate strategic opportunity. }\end{array}$ & $\begin{array}{l}\text { Wharton, Long \& } \\
\text { Schwartz, 2008; } \\
\text { Fairholm, 2009; } \\
\text { Cohen et al., } 2016\end{array}$ \\
\hline & Stakeholders & $\begin{array}{l}\text { Customers, shareholders, suppliers, } \\
\text { employees, the public; groups or } \\
\text { individuals with distinct priorities and } \\
\text { values that shape organizational } \\
\text { trajectories as well as possibilities. }\end{array}$ & $\begin{array}{l}\text { Brammer \& } \\
\text { Millington, 2003; } \\
\text { Smith et al., 2013; } \\
\text { Fernandez \& } \\
\text { Rainey, } 2017\end{array}$ \\
\hline & Vision & $\begin{array}{l}\text { Long-term "organizational intent"; } \\
\text { strategic conversion of organizational } \\
\text { resources to realize values through } \\
\text { action; guiding principle that shapes } \\
\text { organizational culture and strategy. }\end{array}$ & $\begin{array}{l}\text { Tosti \& Jackson, } \\
\text { 2000; Foster \& } \\
\text { Akdere, } 2007\end{array}$ \\
\hline
\end{tabular}

\section{Overview of Research Design and Rationale}

There are quantitative and qualitative dimensions to nearly any kind of organizational process (Chan, 2000). In order to document rapidly evolving phenomena, there are strengths to using multiple methods (Creswell \& Creswell, 2017; Lucero et al., 2018). However, the epistemologies of qualitative and quantitative approaches are profoundly different and these distinctions shape the kinds of question each discipline 
asks, the methods employed, and the kinds of analyses possible. Quantitative methods typically ask "what," "when," "where," and "who," whereas qualitative approaches seek to answer queries regarding "why" or "how" (Kaur, 2016).

Positivism is the general logic of quantitative inquiry whereas other types of logic (e.g., interpretivism, post-positivism) guide qualitative study (Yilmaz, 2013). Further, the approach and types of data collected for each discipline also differ: a quantitative approach estimates relationships among observable and measurable variables using numerical data whereas a qualitative approach provides meaning and perspective using textual or graphical data (Pluye \& Hong, 2014; Yilmaz, 2013). This is not to say that creative quantitative methods can't be employed to analyze visual data, for example, but the type of question as well as nature of the data source generally directs which approach is the most optimal to employ.

As it pertains to implementation research, Palinkas et al. (2014) state that a single methodological approach is often inadequate to sufficiently capture the complex process of incorporating new policies and practice. Further, the authors cite prior work to conclude that mixed methodology is often more capable of providing greater depth and breadth of understanding than either approach (qualitative or quantitative) can do alone. Both methods can be employed to answer the same question "either simultaneously or sequentially...through convergence of results from different sources" (Palinkas et al., 2011, p. 14).

From this perspective, the key research question for this dissertation was best answered through mixed methodology given its emphasis on processes as well as 
outcomes associated with HFEP implementation. The data sources that informed relevant procedural factors were perspectives and insights from key informants (textual data) whereas the data most appropriate to evaluate measurable outcomes were financial reports (numerical data) sourced from the organization's business office. As stated earlier, these types of data have optimal approaches for analysis and interpretation.

The aims, methods, and analyses are summarized in Table 3.3.

Table 3.3 Summary of the Study

\begin{tabular}{|c|c|c|c|}
\hline \multicolumn{2}{|c|}{ Aim } & Method & Analysis \\
\hline $\mathbf{1}$ & $\begin{array}{c}\text { Describe the barriers to and } \\
\text { facilitators of } \\
\text { implementing HFEPs } \\
\text { within six health care } \\
\text { organizations }\end{array}$ & $\begin{array}{c}\text { Two semi-structured } \\
\text { key informant } \\
\text { interviews from six } \\
\text { health care } \\
\text { organizations }(\mathrm{N}=12)\end{array}$ & $\begin{array}{c}\text { Thematic content } \\
\text { analyses derived from } \\
\text { coded (a priori and } \\
\text { inductive) interview } \\
\text { transcripts }\end{array}$ \\
\hline $\mathbf{2}$ & $\begin{array}{c}\text { Evaluate the economic and } \\
\text { behavioral outcomes of an } \\
\text { SSB ban within one health } \\
\text { care organization }\end{array}$ & $\begin{array}{c}\text { Monthly point-of-sale } \\
\text { reports and revenue data } \\
\text { (FY 2012-2019) from 17 } \\
\text { retail food sites }(\mathrm{N}= \\
\text { 1615 site-months) }\end{array}$ & $\begin{array}{c}\text { Baseline descriptive } \\
\text { statistics and interrupted } \\
\text { time series analysis } \\
\text { (linear piecewise } \\
\text { regression model). }\end{array}$ \\
\hline 3 & $\begin{array}{c}\text { Determine organizational } \\
\text { context of six health care } \\
\text { organizations and develop } \\
\text { contextual }\end{array}$ & $\begin{array}{c}\text { Two semi-structured } \\
\text { key informant } \\
\text { interviews from six } \\
\text { health care }\end{array}$ & $\begin{array}{c}\text { Thematic content } \\
\text { analysis; narrative } \\
\text { summary; comparative } \\
\text { analysis to produce } \\
\text { policy recommendations }\end{array}$ \\
\hline
\end{tabular}

\section{$\operatorname{Aim} 1$}

Overview

Aim 1 sought to qualitatively describe the barriers to and facilitators of HFEPs implementation within six health care organizations in Oregon: 1) a state health agency; 2) a regional not-for-profit health care system; 3) an urban teaching hospital; 4) a faith- 
based hospital network; 5) a rural not-for-profit hospital; and 6) a federally-funded health care administration. This first aim had three objectives:

(a) To describe the organizational factors that inhibit the implementation process;

(b) To describe organizational factors that facilitate implementation; and

(c) To compare and contrast the implementation factors cited by executive and operational staff within each organization.

By studying six diverse health care organizations that are in various stages of HFEP implementation, the findings added to the literature with respect to facilitators of and barriers to implementation. Further, by interviewing two strata of leadership and eliciting their experience, the study described nuance between the kinds of factors cited and whether executive leadership and operational staff aligned or diverged in their perspectives of the implementation challenges. This was done for each specific organization as well as compared across organizations.

\section{Qualitative Design and Rationale}

This aim utilized semi-structured key informant interviews and thematic content analysis to determine implementation factors related to HFEP implementation. A constructivist approach guided the data collection and analysis, as this positionality views human experience as a constructed reality, influenced by social, cultural and paradigmatic influences (Arghode, 2012). This aim sought to conceptualize each subject's experience by asking semi-structured and open-ended questions and subsequently coding abstract terms, in order to generate new knowledge as well as 
synthesize previous literature. The construct of the interview guide, coding and analysis was guided by the conceptual framework. Using the literature review as well as the framework for identifying and organizing a priori codes, open-ended questions and probes were constructed in order to study the relevant topics of interest.

These interviews were transcribed verbatim by the researcher and the transcripts were the source data for thematic content analysis. The goal of this analysis was to elucidate the factors that facilitated or inhibited enhancing food environments within health care organizations and the extent to which organizational characteristics shaped the implementation process. After transcribing interviews, the transcripts and memos were analyzed for a priori codes as well as inductive codes that emerged. Charmaz (2008) underscored that data collection and analysis is an iterative process, wherein interpretation and analysis happen in tandem as the researcher reads, codes and interprets results. Baker (2017) emphasized the ongoing process of qualitative data analysis, which "begins in the early stages and continues throughout the study" (p. 90).

\section{Selection of Participating Organizations}

To study the organizational factors that might have hindered or facilitated HFEP implementation among health care organizations, six organizations and twelve respondents were solicited for study participation. Further, identifying context-specific facilitators and barriers experienced during the implementation process required examining multiple units of analyses that are distinct from one another. For this aim, the 
unit of study was each participating interviewee $(\mathrm{N}=12)$. Each respondent possessed institutional knowledge and these perspectives provided the data for qualitative analysis.

In selecting health care organizations to study, the researcher sought to recruit diverse health care organizations within Oregon in order to ensure that a heterogenous group convey their experiences and perspectives - from geographic service area to differences in payer mix, size, governance structure, stakeholder groups and tax status. Responses regarding barriers and facilitators were contextualized to the attributes of each organization so as to inform the general field of health care and provide prospective health systems more clarity in the role of the organizational context when implementing HFEPs.

\section{Inclusion Criteria}

There are 62 hospitals, one state health agency, one federally-funded medical center, and 154 federally qualified health care centers in Oregon (Oregon Hospital Guide, 2019; OAHHS, 2017; OHA, 2012). These health care organizations vary in governance structure, geographic service area, payer mix, size, stakeholder groups and tax status. Of interest to this study are those organizations that have acknowledged the role of health care institutions in enhancing food environments. The following criteria was used to determine study inclusion:

(1) The participating organization had to have (a) attempted or (b) successfully implemented one or more HFEPs (operationalized earlier in this Chapter). This criterion ensured that selected organizations would be able to speak to 
the factors associated with implementation, regardless of the implementation stage each organization was currently in.

(2) Each participating organization must either totally or partially operate in Oregon. This criterion ensured that all included organizations shared the same broader policy context. This criterion also enabled the researcher to conduct face-to-face interviews, when possible, at the participants' host organizations for their convenience.

These criteria were applied through review of the media, grey literature, and websites of health care organizations in Oregon to determine whether policies were in place to enhance retail food environments. Organization selection was done, in part, based on key informant recommendations and review of media coverage. The following list described the six participating health care organizations, including their demographic characteristics, the primary reason for inclusion in the study, and - if there are multiple locations throughout the state - the description of the participating site(s). Sites within organizations were selected on the basis of having one or more retail food venues.

(1) Organization 1 is a state health agency, serving over a million Oregonians every year through facilitation of the state's Medicaid program and providing research, policy guidance, and public services for the state. Most direct services are provided through care-coordinated organizations located across the state whereas research, reporting, and administration of the public health agency are based between two urban headquarters (and sites for this study), located in Portland and Salem. Organization A is a public agency and thus has 
a legislated governance structure. The governing board as well as operational guidelines are controlled by statutes which also mandate inclusion of certain stakeholders and groups. The unique governance structure was of primary interest for inclusion in the study. The participating sites for this study were the two headquarters, located in Portland and Salem. Between the two sites, there were four retail food venues, two cafes as well as two onsite coffee counters at the respective locations. These two buildings were the workplaces of Organization A's nearly 3,000 employees; the food venues were also open to the public.

(2) Organization 2 is a health care system that operates 50 hospitals and $>800$ clinics across five states in the Pacific Northwest, employing over 120,000 employees. This large, non-profit health care system provides full-spectrum medical care, facilitates a health insurance plan, and has a number of affiliations with other health systems. Within Oregon, there are three hospitals, five medical centers, one cancer institute, a cardiac wellness center and a corporate office, with a total of 19 retail food venues among 11 Oregon sites. The diverse scope of services offered and large operational size of this health care system was of interest for inclusion in the study.

(3) Organization 3 is an academic medical center as well as the state's only Tier-1 acute care center. Located primarily in the urban core of Portland, this health care organization is governed by both principles of health care as well as education. With over 16,000 employees serving students as well as patients, 
this organization's dual-role was of interest for inclusion in the study and the influence it may have played in relation to HFEP implementation. The participating sites were the two main hospitals in downtown Portland. Within the main campus, there were seven retail food venues. At the second site, a café and a coffee stand were the two food venues.

(4) Organization 4 is a federally funded medical center. With a little over 4,000 staff, this medical center provides acute care for military veterans. As part of the federal government, the budget, policies and procedures of Organization 4 are heavily centralized and hierarchical. Physically adjacent to Organization 3, the main medical center (and participating site for this study) is located in an urban environment and is the referral center for Oregon, Southern Washington and parts of Idaho. The highly homogenous patient profile and centralized governance structure were two attributes of interest for inclusion in the study. The participating site has one retail food venue.

(5) Organization 5 is a faith-based health care network comprised of one medical center and 34 acute care clinics in the Portland metropolitan area. Employing nearly 2,000 employees, Organization 5 is guided by the principles of the Seventh Day Adventist faith tradition as well as mainstream health care values. The historic religious founding of the health care network still plays a role in the system through a faith-based tradition of vegetarianism and "food as medicine" approach. The ideological/religious guiding principles of this organization made it a valuable organization for study of HFEP 
implementation. The participating site is the main hospital which has two retail food venues.

(6) Organization 6 is a rural hospital. This health care organization is a not-forprofit hospital with 500 employees. Offering a range of medical services, Organization $\mathrm{D}$ is the primary source of medical care for 35,000 Oregonians between Umatilla and Morrow counties, with half of patients either enrolled in Medicaid (35\%) or uninsured (15\%). Thus, the patient/payer mix and rural setting were two characteristics that made Organization 6 a useful organization to include in the study. The participating site was the hospital whose cafeteria comprised the sole retail food venue.

\section{Data Collection}

For each of the six participating health organizations, qualitative semi-structured open-ended interviews were conducted, seeking insight from one executive and one operational manager from each organization for a total of two interviews per site. These two types of individuals were selected given their complementary role in the implementation process. Key informants provided their perspective, identifying facilitators and barriers during implementation as well as describing their organizational context. These interviews took place in-person and ranged between $41-57$ minutes to complete. If an in-person meeting was not possible, the interview was conducted over the phone. Field notes were also created by the researcher to capture relevant thoughts during and immediately after each interview. The interviews were audio-recorded in order to 
ensure accurate capture of sentiments. Though a total of twelve interviews were planned, the inability to conduct an executive-level interview with Organization 6 led to its exclusion from this aim's objectives. Thus, only ten interviews comprised the analytic sample for this aim.

The interviews began by the researcher reiterating the goals of the study and reminding the interviewee of their rights as a subject. Then, respondents detailed specific steps taken to enhance onsite food environments, identifying implementation factors that have affected this process, describing the organizational context of their institution, and discussing strategy formation and environmental pressures (refer to Appendix A for the full interview protocol). The construction of open-ended questions allowed flexibility in response by respondents and served as a forum for respondents to identify factors and processes unique to the organization's site. Goldman and Swayze (2012) describe indepth interview processes with health care elites and caution that a scarcity of time may cut interviews short. To account for this, questions were ordered by priority.

\section{Recruitment}

Prospective interviewees were recruited in two waves: for executive leadership, the researcher queried organizational charts to identify 2-3 potential respondents. Next, the researcher relied on key informant recommendations, faculty recommendations and networking contacts to narrow the candidate pool to the most appropriate contact for each organization. The researcher then relied on the executive contact at each respective site to identify operational managers. This strategy was chosen given that the executive leader 
would be best equipped to identify, and more importantly, connect the researcher to operational staff that may otherwise not respond or decline an outside request for an interview. However, relying on the executive for a recommendation carried a certain risk of bias, namely, the ability for the executive to connect the researcher to operational staff that were politically aligned with the executive, resulting in inaccurate and distorted information. Thus, the researcher asked each executive for a list of "suggested" operational staff and reserved the right to extend an interview request to staff not included on the list. This mitigated the risk of bias as well as ensured that prospective interviewees could opt out of the research participation request.

Prospective interviewees were recruited via electronic mail invitation (Appendix B), which included a consent form approved by the PSU Institutional Review Board (Appendix C). Interviewees were informed of their rights as research subjects in the study, detailing the scope of the inquiry as well as the length of time requested for a scheduled in-person interview. If the prospective respondent did not answer the recruitment email after seven days, a follow-up email was sent. If there was no response to the follow-up email, the researcher selected another person from the list of suggested people provided by the executive. Those respondents who consented to being interviewed were asked to sign two copies of the consent form, one of which was kept by the researcher.

\section{Interview Guide Development}

Semi-structured open-ended interview questions were used to query health care executives and operational managers using an interview guide that was developed based 
on the literature review conducted for Chapter 2, the conceptual framework and a qualitative methodological framework developed by Kallio and colleagues (2016), who developed a discipline-neutral five-step process (Figure 3.1). The rigor of a standardized framework contributed to the objectivity of the questions and bolstered the trustworthiness of this data collection method. 
Figure 3.1. Organizing Framework for Interview Guide Development

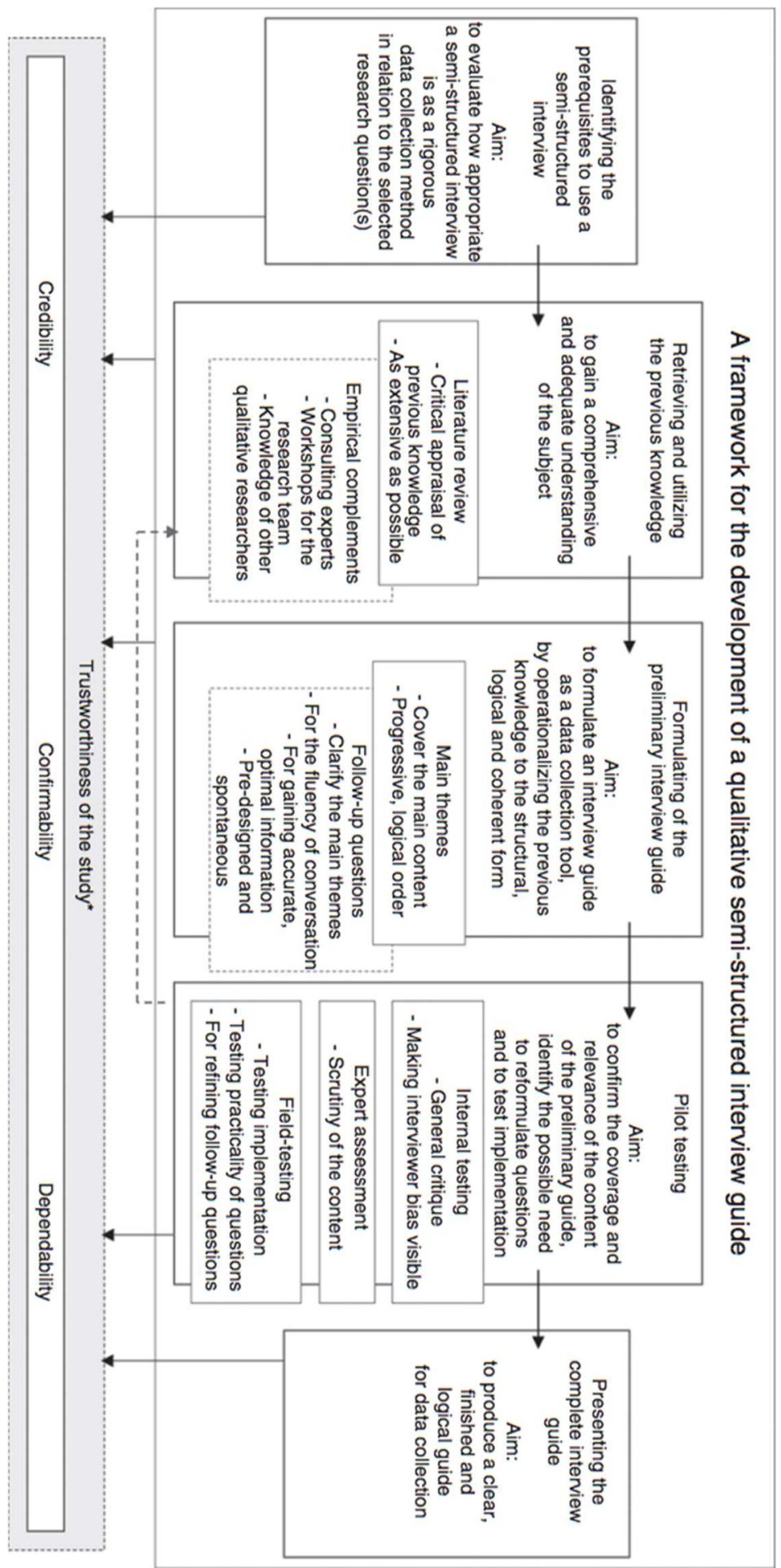

Source: Kallio et al., 2016 
Step 1: Prerequisites. This first step required the researcher to examine the research question of interest and determine the appropriateness of using semi-structured interviews as a data collection method. As described earlier in the chapter, the goal of Aim 1 was to aggregate experiences and perspectives of key informants regarding the process of HFEP implementation. Given that the semi-structured interviews were optimal for gleaning personal insights and that these data would be textual in nature, semistructured interviews were determined to be an appropriate data collection method.

Step 2: Retrieving Previous Knowledge. In order to create a comprehensive interview guide, a review of pertinent literature first took place in order to synthesize the existing evidence base and identify knowledge gaps. More importantly, this step was necessary in order for the researcher to be adequately prepared to ask primary as well as follow-up questions. This researcher conducted an extensive literature review, as presented in Chapter 2, including a review of contemporary obesity prevention strategies; a history of HFEP development in the U.S. and application in the literature; the role of health care institutions in modeling health; and the conceptual role of organizational context in implementation success. The researcher also identified a conceptual framework that provided a guiding lens through which to study the key research question.

Step 3: Formulating the Guide. This step applied Step 2 knowledge to construct a preliminary interview guide, creating questions that "direct conversation toward the research topic during the interview" (Kallio et al., 2016, p. 13). The level of specificity in the interview guide was open-ended enough to allow dialogue throughout the interview and flexible enough to adjust for out-of-order questioning. The researcher was thoughtful 
of the wording of questions and avoided leading questions. Finally, well-formulated questions and follow-up probes were also participant-oriented in order to elicit meaningful and in-depth responses.

The researcher applied existing knowledge to construct the interview questions using the conceptual framework components as main research topics. For the majority of the questions, follow-up probes were created in order to clarify the main question. After initial construction of the interview guide, the researcher reviewed questions for redundancy and consolidated questions that queried similar topics. Next, the researcher ranked questions from highest- to lowest-priority and restructured sections according to priority. This was done to ensure capture of the most relevant information in the case of time constraints during interview sessions.

Step 4: Pilot Testing. This step confirmed the relevance and accuracy of the questions in the interview guide by identifying confusing questions, testing the intelligibility of the guide and improving the ability of the researcher to collect pertinent data. The framework identified three common techniques for validation: (1) internal testing, which directed the researcher to collaborate with a research team to identify inappropriate questions as well as minimize interview bias; (2) expert assessment, which directed the researcher to solicit outside specialists to determine the comprehensiveness of the guide; and (3) field testing, in which the researcher solicited insight from potential respondents by simulating the interview process.

The researcher elected to pilot test the interview guides using all three validation techniques: internal testing, expert assessment and field testing. Regarding internal 
testing, the researcher's dissertation committee provided thoughtful review and critique of the appropriateness of the questions, including the level of specificity and relevance. Regarding expert assessment, faculty with expertise in organizational theory provided feedback as to the comprehensiveness of the questions and relevance to the key research question. Finally, the researcher conducted a mock interview with an executive at a health care system not included in the study to determine actual length of the interview, extraneous or redundant questions, and flow of the interview guide.

Step 5: Presenting the Complete Guide. This last step recommended appending the interview guide in any study paper with the aim of providing readers a mechanism by which to assess the integrity of the interview guide questions. The additional goals of transparency and reproducibility bolster the trustworthiness of the research. Given that the interview guide was part of the dissertation study, the researcher has appended the guide in the finalized dissertation (see Appendix A) as well as future publications.

\section{Summary}

The use of a standardized framework to create a robust interview guide ensured that questions would comprehensively probe the respondent's perspective during HFEP implementation as well as insight regarding the organizational context in shaping implementation challenges. The benefit of using this data collection method was its capacity to measure complex issues and present rich descriptions. Pilot testing the interview guides via internal testing and expert assessment confirmed the comprehensiveness and appropriateness of the questions as well as streamlined the guide. The goal of these interviews, as described earlier, was to query the respondents of their 
perceived realities in which factors affected the process of enhancing food environments; to this end, the interview guide was participant-oriented, flexible, and open-ended, containing probes to clarify main questions and research topics. Table 3.4 displays the interview questions as they relate to the conceptual framework. 


\section{Table 3.4 Conceptual Domains and Interview Questions}

\begin{tabular}{lll}
\multicolumn{1}{c}{ Concept } & \multicolumn{1}{c}{ Operational Definition } & \multicolumn{1}{c}{ Question } \\
Healthy Food & Any combination of the following policies & Please describe the policies and \\
Environment & instituted within the retail environment: (1) trans- & $\begin{array}{l}\text { procedures regarding your } \\
\text { Policies }\end{array}$ \\
fat and/or sodium limits; (2) limiting or banning & organization's food and beverage \\
(HFEPs) & access to sugar-sweetened beverages; (3) & $\begin{array}{l}\text { environment. Describe } \\
\text { procurement practices related to }\end{array}$ \\
& instituting serving size limits; (4) prioritizing & $\begin{array}{l}\text { these new practices. Has your } \\
\text { healthy methods of food preparation (e.g. }\end{array}$ \\
& removing fryers); (5) nutrition labelling for meals & organization trained staff or \\
& and items at point of service; (6) promotion of & provided the public with \\
healthy foods and beverages in marketing & materials?
\end{tabular}

Organizational Characteristics including structure (governance, context

size, interorganizational coupling), receptivity (tension for change, norms and culture), resource availability (slack resources, social network location, information sharing), and leadership (change agents, stakeholders, vision).

Environmental context

Adoption decision

Implementation
Felt needs; norms of the social system; broader systems that shape external threats and institutional pressures.

Steps and/or decisions made to implement healthy food environment policies.

Any deliberately initiated attempt to introduce new patterns of action in health care or some other formal organizational setting. Deliberate initiation means that an intervention is: institutionally sanctioned; formally defined; consciously planned; and intended to lead to a changed outcome.

Negative, positive or neutral impact on revenue; changes in consumer purchasing behavior.
How do the goals of your organization's stakeholders align or diverge? What resources and communication channels are available? Describe the degree of interorganizational cooperation needed for your organization to achieve goals? Was the change to enhance food environments championed by any particular individual?

What short- and long-term external pressures do you feel your organization needs to address? Does your organization have a strong impetus to "model health"?

What events or actions led you to consider making changes to the internal food environment?

Please describe the specific steps taken by your organization in enhancing the food environment. Has your organization surveyed opinions of staff or piloted any changes? Have there been any barriers in enhancing the food environment? What have been facilitators that have aided this process?

Have you instituted monitoring and evaluation efforts for these new practices? Will sales data inform procurement practices? 


\section{Data Analysis}

Data analysis and interpretation are overlapping, but distinct, processes (Baker, 2017). More specifically, data analysis requires the breaking down of information into principal parts whereas interpretation produces meaning and understanding of the findings. Data analysis was the culminating step in a process consisting of design, preparation, and data collection. For this aim, interviews were audio-recorded and identifying information was removed. Both the interview and any field notes created during the interview were transcribed by the researcher within five days of each conversation to preserve and represent sentiments accurately. All materials offered during the discussion were included in the coding and thematic analysis. Upon transcribing each interview, the transcript was reviewed by the researcher in full before any coding took place. The use of parent-child codes was employed (also known as super-ordinate and sub-ordinate levels of coding) (Basit, 2003). Initial coding of broad categories (parent) were followed by smaller sets of code for subsequent rounds (children). As qualitative data analysis is an iterative process, multiple passes between large concepts and specific codes took place to describe and refine code families. The researcher coded any words or phrases that directly corresponded to operationalized key concepts (a priori codes) from the conceptual framework, a form of deductive analysis. Lastly, inductive analysis was conducted — the rationale for utilizing both forms of analysis (deductive/a priori and inductive) was that, while the literature and conceptual framework may have informed possible factors related to the implementation process, the researcher avoided relying too heavily on the framework alone in order to minimize bias. Instead, the literature review 
and conceptual framework provided possible coding terms whereas inductive analyses elucidated any unexpected or unique factors that emerged. Interview transcripts were managed and stored in NVivo, a qualitative analysis software, for coding and thematic content analysis.

Upon completion of the thematic analyses for all organizations, the researcher presented the findings along with the Consolidated Criteria for Reporting Qualitative Research (COREQ) (Appendix D), a formal reporting guideline consisting of 32 items describing the selection, methods, analysis and interpretation of the findings (Tong, Sainsbury \& Craig, 2007). Similar to the CONSORT reporting guideline for randomized control trials (Moher et al., 2001) or STROBE for observational studies (Von Elm et al., 2007), COREQ represents an equivalent guideline for reporting qualitative research, specifically interviews and focus groups. This checklist will also be included upon submission of this aim for refereed publication.

\section{$\operatorname{Aim} 2$}

Overview

Aim 2 sought to quantitatively evaluate the economic and behavioral outcomes of a sugar-sweetened beverage ban established within one health care organization, a notfor-profit health care system. Whereas the first aim queried an array of diverse organizations to elucidate implementation factors, this second aim conducted a more thorough examination of the revenue effects and consumer behavior changes of one 
HFEP that has been growing in popularity and uptake. This second aim had three objectives:

(a) To compare monthly gross sales of all beverage categories before (FY 20122014) and after (FY 2015-2019) the SSB ban;

(b) To assess the percent change in sales by beverage type before (FY 2012-2014) and after (FY 2015-2019) the SSB ban; and

(c) To describe the stratified impact of the ban on venues that differed by size, type and geographic location.

For each objective, point-of-sale reports and monthly revenue data from the health care system's business office provided the source data. This revenue data captured pre- (20122014) and post-implementation (2015-2019) sales data. Data were sourced from 19 retail food sites within the health care system.

An interrupted time series design evaluated changes in sales (Objective (a)) through segmented regression analysis (SRA). Gross revenue was reported over time and Objective (b) used subgroup analyses within the interrupted time series design, estimating pre- and post-implementation sales trends for each of the seven beverage types: water, juice, milk, coffee, tea, sports/energy drinks, diet SSBs. Diet SSBs remained available after implementation of the SSB ban in 2015. For Objective (c), stratified analyses evaluated the differential impact of the initiative with covariates such as food outlet type (café, cafeteria, coffee cart), venue size based FY 2012 gross revenue (small, $<\$ 50,000$; medium, $<\$ 100,000$; large, $>\$ 100,000$ ), and venue location (urban, suburban, rural). 
Describing changes in consumer behavior (Objective (b)) would be useful for the host organization as well as prospective organizations that are considering a sugarsweetened beverage ban but are unsure of what to supply in order to meet shifting consumer demands and subsequent purchasing behavior.

\section{Quantitative Research Design}

The second aim had three objectives, all of which used numerical data derived from financial reports to evaluate economic and behavioral consequences of the SSB ban. For the first objective, an interrupted time series analysis (ITSA) evaluated gross beverage sales to determine if restricting SSB sales had a significant impact on gross revenue. ITSA is a quasi-experimental study design in which equally spaced time points before and after a policy change are analyzed using segmented regression to estimate the changes in level and slope in the post-intervention period as compared to the preintervention period. The regression analysis estimates interaction terms between implementation of a policy and time, which was the goal of this objective. Further, the available data met the critical requirements of ITSA. First, the intervention needed to have occurred at a clearly defined point in time, which is the first month of 2015. Second, ITSA required the availability of robust, high quality data before and after the interruption. The dataset received contained consistent and robust data that spanned from January 2012 to October 2019. Given this work met these ITS assumptions, the following strengths, limitations and threats to validity of ITSA are discussed.

One strength of ITSA is its robust approach when randomization is not possible or ethical. This approach is best for natural experiments or observational data, which is the 
kind of data used in this aim. Additionally, ITSA is able to control for secular trends in the data, unlike a two-period before-and-after $t$ test, by using multiple pre-intervention time points to estimate a regression line and detect if the trend has a down- or up-ward slope independent of the intervention (Penfold \& Zhang, 2013). The relative ease of conducting stratified analyses to evaluate differential impact of the SSB ban on, for example, urban versus rural sites was another strength of this approach. Finally, in considering what would have been the most useful deliverable for the partner organization, ITSA provides clear and easy to interpret graphical results. Even before regression modeling, the visual component (see Chapter 5) was useful in assessing the outcomes and communicating results to relevant stakeholders.

Potential limitations to ITSA include not having enough pre-intervention timepoints, with some stating a need for a minimum of eight timepoints (Bernal et al., 2017) in either direction in order to have sufficient power to estimate regression coefficients. In this study, there were 24 pre-intervention timepoints and 58 postintervention timepoints. The main limitation of ITSA in this study was the lack of a matched comparator group (Biglan et al., 2000). Selecting a matched control population and conducting a difference-in-difference analysis could have countered this limitation. However, an adequate comparison group did not exist.

Finally, there are three threats to validity in ITSA. The first is history/competing interventions that coincide during the intervention of interest, as ITSA is only valuable when the policy of interest is the only change in the setting. There were no known competing interventions in this study. The second threat is a change in instrumentation or 
an inability to measure the outcome of interest. The researcher received confirmation of the availability of follow-up data and confirmation that follow-up data have not changed in format. Finally, selection bias can pose a threat to validity of the findings. However, selection is not a factor in single-group ITSA because the same organization will be under study before and after the intervention. Additionally, selection bias can also emerge from incomplete data entry; the researcher excluded two sites with incomplete data to ensure data completeness, for a total of 17 sites with 1,615 site-months (unit of analysis).

\section{Selection of the Organization}

This organization was chosen for reasons of data quality and access. First, the researcher was able to gain access to the dataset through a concerted nine-month process of inquiry. Second, the SSB ban was implemented at a specific point in time, January 2015, enabling a quasi-experimental design to study the impact of the ban. Within Oregon, there are three hospitals, five medical centers, one cancer institute, a cardiac wellness center and a corporate office, with a total of 17 retail food venues among these 11 participating sites, which span all over the state. Table 3.5 describes the sites, food venue type (cafeteria/café/coffee cart) and geographic location of the service area.

Table 3.5 Description of Organization's Food Sites

\begin{tabular}{ccccc} 
Site & Type & Location & Size & FY2012 Revenue \\
1 & Cafeteria & Urban & Large & $\$ 281,041$ \\
2 & Cafeteria & & Large & $\$ 444,938$ \\
3 & Cafe & & Medium & $\$ 233,279$ \\
4 & Cafeteria & \multirow{2}{*}{ Urban } & Medium & $\$ 188,777$ \\
5 & Café & & Large & $\$ 312,802$ \\
6 & Cafeteria & Suburban & Small & $\$ 15,045$ \\
7 & Coffee cart & & Small & $\$ 149$ \\
8 & Cafeteria & Suburban & Small & $\$ 40,150$ \\
& & & & \\
& & & 103 &
\end{tabular}




$\begin{array}{ccccc}9 & \text { Coffee cart } & & \text { Medium } & \$ 61,129 \\ 10 & \text { Cafeteria } & \text { Rural } & \text { Small } & \$ 49,102 \\ 11 & \text { Cafeteria } & \text { Rural } & \text { Small } & \$ 39,459 \\ 12 & \text { Cafeteria } & \text { Suburban } & \text { Medium } & \$ 69,677 \\ 13 & \text { Cafeteria } & & \text { Large } & \$ 402,093 \\ 14 & \text { Café } & & \text { Small } & \$ 47,458 \\ 15 & \text { Coffee cart } & \text { Suburban } & \text { Large } & \$ 411,177 \\ 16 & \text { Café } & & \text { Large } & \$ 594,971 \\ 17 & \text { Cafeteria } & \text { Suburban } & \text { Medium } & \$ 69,685\end{array}$

\section{Data Collection}

Secondary data, in the form of monthly financial reports, was the source data in evaluating the economic and consumer behavior outcomes of the SSB ban. The organization's data warehouse was queried by a dedicated staff analyst who pulled relevant information, including food venue names, venue type, physical location, and revenue reports. The extracted data was relayed in the form of a .CSV file which was uploaded to a Microsoft Excel spreadsheet and transferred and managed using Stata software version 16 (StataCorp, College Station, Texas). All files were stored on a password-protected computer.

\section{Data Analysis}

Monthly sales reports were pulled by the health care organization's Oregon region planning analytics department. Additionally, three covariates were also pulled for each included site: (1) food venue type, which was coded as café, coffee-cart, or cafeteria; (2) geographic location of venue, which was coded as urban, suburban, or rural; and (3) food venue size, which was coded as small, medium, or large. Food venue types were determined in the following way: for each food site containing one food venue, that 
venue will be coded as the cafeteria. For sites with multiple food venues, the venue with the largest annual revenue in FY 2012 was coded as the cafeteria for all years going forward. All other food venues within that site were coded as cafés (excluding coffee carts). Distinguishing cafés from cafeterias attempted to preserve granularity in order to compare similar food venues. Geographic location was determined by cross-comparing the site location zip code to the 2010 US Census Tract data. Finally, site size was determined by using each venue's FY 2012 gross revenue to categorize into small $(\$<50,000)$, medium $(\$ 50,000-\$ 100,000)$, and large $(\$>100,000)$ venues.

For Objective (a), an interrupted time series study design compared gross beverage revenue before and after implementation of the SSB ban ("intervention") in January 2015. This was done by estimating the changes in level and trend through SRA (Penfold \& Zhang, 2013). Fitting a least squares regression line before and after January 2015, the month in which the nutritional initiative went into effect (serving as the "interruption" in the interrupted time-series design), determined the regression coefficient estimating the pre-intervention slope (2012-2014), the change in level at intervention (first three months in 2015 [lag time]), and the change in slope from pre- to postimplementation (2012-2019). The researcher consulted with the dissertation committee for additional methodological considerations, such as correcting for autocorrelation and sensitivity testing, to ensure robust modeling.

For Objective (b), point-of-sale reports were examined and coded for various beverage names. Beverage names were categorized into one of the seven types: coffee, tea, milk, juices, sports/energy drinks, water and diet SSBs. This categorization was 
verified by the internal analyst. Unknown point-of-sale items were also directed to the analyst for further clarification. Upon completion of beverage categorization, the researcher replicated the interrupted time series analysis done for Objective (a) for each

of the seven beverage types, estimating changes in level and trend through SRA by fitting a least squares regression line before and after January 2015. Findings were reported graphically to present which beverages decreased or increased in consumption following the sugar-sweetened beverage ban (see Chapter 5).

For Objective (c), following the SRA for gross beverage revenue trends, stratified analyses were conducted in order to evaluate the differential impact of the ban on rural, suburban, and urban food venues, the differential impact by venue size, and the impact by venue type. For all analyses, graphical presentation of the data was generated.

\section{$\operatorname{Aim} 3$}

Overview

Aim 3 sought to describe the relationship between organizational contexts and HFEP selection within six health care organizations, with an objective to develop context-informed policy recommendations. This final aim had three objectives:

(a) Determine the organizational context of each organization;

(b) Describe the relationship between contexts and HFEPs adopted within each organization; and

(c) Develop context-informed policy recommendations for HFEP implementation. 


\section{Mixed Methods Study Design}

Within mixed methods (MM) study designs, the order in which qualitative or quantitative research aims are undertaken will affect subsequent methodological appraisal. Four common types of MM designs exist: a sequential explanatory design, whereby quantitative work is followed by qualitative inquiry; a sequential exploratory design, whereby qualitative inquiry is followed by quantitative work; a triangulation design, wherein qualitative and quantitative are done simultaneously to offer distinct analyses; and an embedded design, where the qualitative and quantitative components are done concomitantly with the focus to support qualitative inquiry with quantitative substudy measures (Ivankova, Creswell, \& Plano-Clark, 2007).

In orientation to the key research question, Aims 1 and 3 proposed to examine implementation barriers and facilitators of adopted HFEPs, and how the organizational context influenced HFEP adoption. Aim 2 proposed to evaluate revenue effects of an SSB ban, as fear of revenue loss is cited as a barrier in the literature. Given that Aim 2 sought to better understand one specific practice in a single institution whereas the qualitative inquiries cover a breadth of factors across organizations, a MM embedded design was an appropriate orientation to the qualitative-quantitative-qualitative sequence of the study.

\section{Distinction between Aim 1 and Aim 3}

Given that the qualitative aims share similar data collection methods, there are some key differences that distinguish these aims as separate endeavors. First, as a matter of orientation, Aim 1 studied the "process" of HFEP implementation by analyzing the 
facilitators of and barriers of each organization. Separately, Aim 3 focused on the "structure" of organizations in selecting HFEPs. Second, Aim 1 had a more practiceoriented emphasis — discussing operational factors — whereas Aim 3 was rooted in a theoretical emphasis of organizational characteristics shaping HFEP selection. This dovetails into the third distinction, which is intended audience: Aim 1 will be submitted to a practice journal where the readership is primarily health care administrators and other operational staff. Aim 3, however, will be submitted to an implementation science journal where the audience skews more toward academic audiences. Fourth, since Aim 1 had the objective of contrasting executive and operational responses, Organization 6 had to be excluded since only one interview was completed with the operational manager. Aim 3 included all six organizations into its analyses. Fifth, the objectives of the aims were unique: Aim 1 measured HFEPs, facilitators and barriers of implementation, and leadership perspectives as to the challenges and opportunities. Aim 3 scored each organization's context, developed narrative summaries to discuss HFEP relationship, and created context-informed policy recommendations based on the findings. Lastly, though the instrument of data collection was the same for both aims (interview protocol), the questions that comprised the textual data for each objective was mutually exclusive: Aim 1 data was based on answers to Questions 4 through 7, whereas Aim 3 data came from Questions 9 through 15. Given these reasons, the scope for each aim is sufficiently original as to warrant separate inquiries (Table 3.6).

Table 3.6 Distinctions Between the Qualitative Aims

\begin{tabular}{|l|c|c|}
\hline & Aim 1 & Aim 3 \\
\hline Protocol Section & Qs 4-7 & Qs 9-15 \\
\hline
\end{tabular}




\begin{tabular}{|l|c|c|}
\hline Orientation & $\begin{array}{c}\text { Process (e.g., facilitators of } \\
\text { implementation) }\end{array}$ & $\begin{array}{c}\text { Structure (e.g., context shaping } \\
\text { HFEP selection) }\end{array}$ \\
\hline Emphasis & Praxis & Theory \\
\hline Audience & $\begin{array}{c}\text { Health care administrators, } \\
\text { operations }\end{array}$ & $\begin{array}{c}\text { Implementation scientists, } \\
\text { organization theorists }\end{array}$ \\
\hline Sample Size & 5 organizations $(\mathrm{N}=10)$ & 6 organizations $(\mathrm{N}=11)$ \\
\hline Objectives & $\begin{array}{c}\text { Measure HFEPs, facilitators and } \\
\text { barriers; contrast perspectives }\end{array}$ & $\begin{array}{c}\text { Determine context; examine } \\
\text { relationship with HFEPs }\end{array}$ \\
\hline
\end{tabular}

\section{Determining Organizational Context}

There were three components when determining organizational contexts among the six participating organizations. First, each participating organization was scored along the four domains: structure, receptivity, resource allocation and leadership. Adopting Krein and colleagues' (2010) coding scheme, each domain was assessed with a positive $(+)$, negative (-), or mixed (+/-) score (see Table 3.7). Scores were derived based on respondent answers to corresponding questions and cited with supporting data (Table 6.7). A positive score for an organizational characteristic would be interpreted as a facilitator of HFEP implementation. While the ranges of $(-)$ to $(+)$ mirrored a generally continuous unfavorable-to-favorable spectrum for HFEP development, some ranges indicated categorical responses (e.g., norms and culture, size). As such, generation of a cumulative score for all domains would not be valid. Instead, the aim of these scores was to serve as heuristic devices for sensemaking during data analysis.

\section{Table 3.7 Scoring for Organizational Context}

\section{Structure}

Governance adaptive, casual

$$
(+)-(+/-)-(-)
$$

rigid, formal 
Size

Interorg. Coupling

Receptivity

Tension for Change

Norms and Culture

Resource Availability

Slack Resources

Social Network Location

Information Sharing

Leadership

Change Agents

Vision

Stakeholders small

loose, mild

$(+)-(+/-)-(-)$

$(+)-(+/-)-(-)$

large

tight, intense

low

uniform

$(+)-(+/-)-(-)$

high conflict

$(+)-(+/-)-(-)$ heterogenous values

unavailable

$(+)-(+/-)-(-) \quad$ available cushion

diffuse

$(+)-(+/-)-(-)$

$(+)-(+/-)-(-)$ concentrated reliable, open absence

weak

aligned, few
$(+)-(+/-)-(-)$ multiple champions

$(+)-(+/-)-(-)$

$(+)-(+/-)-(-) \quad$ conflicting, many

There are a few notes of consideration. Namely, as demonstrated above, the weights differed by the nature of the variable. Each value was not be aggregated to calculate a cumulative score. Further, a "+" value did not indicate that a factor is insignificant for the particular organization. Rather, these factors will be plotted initially by the researcher after analyzing thematic findings from Aim 1.

Data Analysis

After scoring each organization's context, discourse analysis was utilized to examine the HFEP relationship. The product of this analysis was a narrative summary of each of the six organizations. Each summary included a brief description of HFEPs adopted followed by a discourse of relevant organizational elements that were favorably or unfavorably scored. The narrative summaries were, in turn, the source material for comparative analysis and the creation of context-informed policy recommendations. The 
goal of this Aim, and overall study, was to go beyond identifying "top factors" to consider and instead describe how organizational characteristics (e.g., structure, resource availability) created environments in which a factor was a barrier in one context and a facilitator in another.

These recommendations were included in dissemination packets that were individually created for each organization. Each organization received a customized final report consisting of a narrative summary of their specific implementation process, the deidentified findings of peer participants, and the context-specific policy recommendations. 


\section{CHAPTER 4: IMPROVING THE FOOD ENVIRONMENT: DETERMINING THE ROLE OF LEADERSHIP PERCEPTIONS ON IMPLEMENTATION CHALLENGES}

\section{Introduction}

Rates of overnutrition and obesity in the US have escalated dramatically throughout the past forty decades, resulting in an ongoing obesity epidemic (Flegal et al, 2016). While the causal pathways of obesity development are complex, a major driver of this public health crisis is obesogenic food environments, characterized by nutrient-poor and energydense foods that saturate the collective physical, economic and sociocultural conditions that influence nutritional status (Hall, 2018; Swinburn et al., 2015). Among a spectrum of obesity interventions, population-level policies aimed at improving the food environment offer a promising start in addressing this epidemic (Stevens et al., 2017; Vandevijvere et al., 2015; Sallis et al., 2009). Examples of policies that target the food environment include implementing rigorous nutritional standards in food and beverage offerings, prioritizing healthy methods of food preparation (e.g. steaming versus frying), using pricing strategies to incentivize healthy food purchases, promoting and marketing nutritious offerings, and establishing healthy vending policies (INFORMAS, 2014).

Food environments in organizations such as hospitals and public health agencies warrant special consideration given their health-focused mission (PHLC, 2013; AHA, 2012). Though there are a number of strategies that health care organizations have taken to address the obesity epidemic, it is noteworthy that many of these same organizations do not have healthy food environments within their retail cafés and cafeterias (Champ et 
al, 2019; Bell et al, 2013; Malhotra, 2013; Lawrence et al, 2009; McDonald et al, 2006). Improving food environments within health care settings has been highlighted by the Centers for Disease Control and Prevention (CDC) as one of seven key strategies to prevent and manage obesity (CDC, 2019). However, most of the refereed literature examining healthy food environment policies (HFEPs) within hospitals focuses on the inpatient dietary environment (Marshall et al., 2012; Pedersen et al., 2012; Brantley, 2009), leaving a paucity of information on facilitators of or barriers to implementation within the retail food venues of these organizations, where hospital staff and visitors regularly eat. Further, the application of HFEPs is often limited, focusing narrowly on one or two specific policies (Palmedo \& Gordon, 2019; Hartigan et al., 2017; Lessard et al., 2014; Eneli et al., 2014; Bell et al., 2013; Block et al., 2010).

Factors that span multiple levels, from environmental facilitators (e.g., institutional pressure, public criticism) (Powell \& DiMaggio, 2012) to inter- and intrapersonal facilitators (e.g., champions, communication channels) (Fairholm, 2009) can influence the process by which retail venues incorporate healthier practices. Specifically, given that decisions to improve food environments are made at the administrative level, leadership perceptions surrounding implementation are particularly relevant to examine (Shill et al, 2012). While the implementation science literature has examined the role of leadership perceptions of implementation challenges in shaping the actual implementation process (Rodriguez et al, 2018), little has been published by way of examining multiple leadership perspectives, such as those of operational managers and executives, who are both involved in decision-making to improve retail food venues. The 
degree to which perception discrepancies aggravate implementation challenges is unknown.

The health care retail food environment differs from the inpatient setting in significant ways. First, unlike inpatient dietary standards, food service operations are not monitored by the Joint Commission on Accreditation of Healthcare Organizations (JCAHO) and thus have no oversight from an accreditation perspective (JCAHO, 2020). Further, retail values are not bound by the need of inpatient settings to serve convalescing patients multiple meals that comport with recommended daily allowances. Finally, most retail food service operations need to meet sales goals with a budget that is separate from inpatient operations. Given these distinguishing characteristics, facilitators and barriers identified in the extant literature may lack the validity and transferability to be transposed from the inpatient setting to the retail context. Thus, studying organizational facilitators and barriers specific to this setting is warranted.

In this study, we sought to qualitatively describe the barriers to and facilitators of HFEP implementation across five distinct health care organizations: 1) a state public health agency; 2) a regional not-for-profit health plan and delivery system; 3) a tier-1 academic medical center; 4) a federally-run medical center; and 5) a faith-based hospital network. We interviewed two levels of leadership, operational managers and executive leadership, within each organization in order to describe similarities and differences between the kinds of factors cited and determine whether executive leadership and operational managers aligned or diverged in their perspectives regarding HFEP implementation challenges. By studying five diverse health care organizations, the 
findings add to the implementation science literature with respect to facilitators and barriers of HFEPs in the retail environment. Further, by interviewing two leadership levels within those organizations, this study describes nuance between the kinds of implementation factors cited based on those perception differences and examines the relationship between perception and organizational performance (Lord \& Maher, 2002).

\section{Methods}

\section{Design and Setting}

This work is part of a broader mixed methods study examining implementation challenges in improving health care food environments. To examine a wide spectrum of HFEP implementation challenges, we recruited health care organizations in Oregon that differed by key characteristics. We prioritized differences in payer mix, size, governance structure, stakeholder groups and tax status in order to ensure that a heterogenous group convey their experiences. We limited recruitment to a single state to ensure a shared public policy context among enrolled organizations.

We identified six health care organizations that adopted healthy HFEPs, recruiting interview participants by email using a purposive sampling strategy. Semi-structured key informant interviews were completed with operational managers and executive leadership who either self-identified as having participated in HFEP implementation at their institution or were identified by others at their institution as having direct knowledge. These two roles were selected given their complementary role in the implementation process, as executives typically make the adoption decision while operational managers 
contend with the logistical variables of execution and maintenance. Ethical approval for this study was obtained from Portland State University Institutional Review Board (Study \#196741-18).

\section{Analytic Sample}

Out of the six health care organizations identified, one organization-a rural non-profit hospital — was excluded due to the unavailability of executive participation in our study. We completed two interviews (one each with an operational and executive staff member) in the remaining five health care organizations for a total of ten interviews.

\section{Data Collection}

We used semi-structured key informant interviews and supplemental document review to explore facilitators of and barriers to implementing HFEPs in participating organizations. Interviews were 41-57 minutes in length and were conducted between December 2019 and March 2020. All but two interviews were conducted in-person, with the interview respondent selecting a private location of their choice (e.g., office, conference room). Interviews were electronically recorded and the interviewer took contemporaneous field notes during each interview. Each participant provided written consent ${ }^{1}$ and was given a copy of the questions in advance of the interview, which was developed using Kallio and colleagues' (2016) five-step protocol framework (see Figure 3.1). Before the start of each interview, participants were notified that they could request commercially sensitive or

\footnotetext{
${ }^{1}$ The two virtual respondents provided written consent by emailing a signed copy of the consent form.
} 
proprietary information be stricken from the transcript record; each participant was provided with their transcribed comments for review of sensitive information. Interviews were transcribed verbatim and all data files (interview transcripts, field notes and any supplemental documentation offered) were managed in NVivo 12 (QSR International, Cambridge, MA) qualitative analysis software.

\section{Identifying HFEPS}

In the interview protocol, respondents were asked to specifically outline HFEPs that their institution adopted, including pilots or trial runs to demonstrate HFEP feasibility, staff procedures, changes to the physical environment, vending changes and any educational materials developed for staff and consumers (see Questions 4A-F, 7A-C, and 8B in Appendix A). Each respondent was then asked to enumerate any HFEP barriers experienced, such as stakeholder resistance or revenue loss, as well as HFEP facilitators, such as champions or staff buy-in (Questions 5A-D and 6A-D). Interview probes were offered to capture all possible HFEPs and each practice's stage in implementation; these interview probes were informed by the Healthy Food Environment Policy Index, an index created by INFORMAS, an international collaborative effort sponsored by the World Health Organization, whose objective was to develop typologies of actions aimed at enhancing the food environment (INFORMAS, 2014). Practices such as nutritional labeling, limits on fat or salt content, healthy vending policies and market promotion were included as probes. For time efficiency, respondents were invited to provide supplemental documentation to be included as part of data collection materials. 
Employing a directed content analysis approach (Hsieh \& Shannon, 2005), an a priori codebook was developed based on a literature review for the broader mixed methods study. Two domains of inquiry included specific HFEPs adopted, as well as facilitators and barriers to implementation. Five iterative cycles of coding took place, with the first round dedicated for transcript review before any coding took place; the second for deductive coding using the codebook; the third round for inductive coding of emerging concepts; the fourth round for reconciling duplication and similarities among codes and consolidating concepts; and the fifth for grouping of codes into categories and, ultimately, themes. For the final two rounds, the use of parent-child codes was utilized to initially create highly specific codes which were then collapsed into broader categories.

Upon completion of the thematic analyses for each interview respondent, coding themes among operational and executive interviews within each organization were compared to identify any differences found between the two roles. This was done separately for each of the five organizations and then across organizations. A single coder approach was used given the broader nature of this study (dissertation research); dissertation committee members provided oversight during qualitative analysis. Upon primary analysis, 27 facilitators and 30 barriers were identified; iterative rounds of analysis generated 6 and 5 categories for facilitators and barriers, respectively. Quote attributions include organization identifier (Table 4.2), followed by an "E" for executive leadership or "O" for operational manager (e.g., [1E] or [4O]).

The study methods and findings are also reported using the Consolidated Criteria for Reporting Qualitative Research (COREQ), a formal reporting guideline consisting of 32 items describing the selection, methods, analysis and interpretation of the findings 
(Tong, Sainsbury \& Craig, 2007). Similar to the CONSORT guideline for randomized control trials (Moher et al., 2001) or STROBE for observational studies (Von Elm et al., 2007), COREQ represents a guideline for reporting qualitative research, specifically for interviews and focus groups (see Appendix D).

\section{Results}

\section{Descriptive Characteristics}

Descriptive information of the five health care organizations and ten interview participants is presented in Table 4.1. Basic demographic information was obtained from the interview transcripts, supplemental document analysis, and a review of each organization's public website. Median employment length among operational managers was 5 years ( 7 months -15 years) while executive leaders were employed a median of 22 years (6 years- 35 years).

\section{Table 4.1 Descriptive Characteristics of Organizations and Respondents}

\begin{tabular}{|c|l|l|l|l|l|l|}
\hline Identifier & $\begin{array}{l}\text { Description of health } \\
\text { care organization }\end{array}$ & Type & $\begin{array}{l}\text { No. } \\
\text { food } \\
\text { venues }\end{array}$ & $\begin{array}{l}\text { No. } \\
\text { employees }\end{array}$ & $\begin{array}{l}\text { Title of } \\
\text { operational } \\
\text { manager }\end{array}$ & $\begin{array}{l}\text { Title of } \\
\text { executive } \\
\text { leader }\end{array}$ \\
\hline 1 & $\begin{array}{l}\text { State public health } \\
\text { agency with legislated } \\
\text { budget and } \\
\text { stakeholders }\end{array}$ & $\begin{array}{l}\text { Government } \\
\text { agency }\end{array}$ & 4 & 3,000 & $\begin{array}{l}\text { Policy } \\
\text { Specialist }\end{array}$ & $\begin{array}{l}\text { Chronic } \\
\text { Disease } \\
\text { Division } \\
\text { Manager }\end{array}$ \\
\hline 3 & $\begin{array}{l}\text { Regional non-profit } \\
\text { health plan and } \\
\text { delivery system }\end{array}$ & $\begin{array}{l}\text { Health plan } \\
\text { and hospital } \\
\text { system }\end{array}$ & 19 & 21,000 & $\begin{array}{l}\text { Retail } \\
\text { Dietician }\end{array}$ & $\begin{array}{l}\text { Regional } \\
\text { Chief } \\
\text { Executive } \\
\text { Officer }\end{array}$ \\
\hline 4 & $\begin{array}{l}\text { Urban non-profit } \\
\text { academic medical } \\
\text { center, sole tier 1 acute } \\
\text { center in Oregon }\end{array}$ & $\begin{array}{l}\text { Hospital } \\
\text { system }\end{array}$ & 9 & 17,000 & $\begin{array}{l}\text { Sustainable } \\
\text { Food } \\
\text { Programs } \\
\text { Manager }\end{array}$ & $\begin{array}{l}\text { Food \& } \\
\text { Nutrition } \\
\text { Services } \\
\text { Director }\end{array}$ \\
\hline $\begin{array}{l}\text { center with centralized } \\
\text { governance and } \\
\text { homogenous patient } \\
\text { mix }\end{array}$ & $\begin{array}{l}\text { Health care } \\
\text { administration }\end{array}$ & 1 & 4,000 & $\begin{array}{l}\text { Retail Food } \\
\text { Services } \\
\text { Director }\end{array}$ & $\begin{array}{l}\text { Associate } \\
\text { Director }\end{array}$ \\
\hline
\end{tabular}




\begin{tabular}{|c|l|l|l|l|l|l|}
\hline 5 & $\begin{array}{l}\text { Faith-based health care } \\
\text { network with } \\
\text { vegetarianism as part } \\
\text { of faith tradition }\end{array}$ & $\begin{array}{l}\text { Hospital } \\
\text { system }\end{array}$ & 2 & 2,000 & $\begin{array}{l}\text { Food } \\
\text { Services } \\
\text { Director }\end{array}$ & $\begin{array}{l}\text { President/ } \\
\text { CEO }\end{array}$ \\
\hline
\end{tabular}

\section{Spectrum of HFEPS Adopted}

For each organization, respondents described all of the HFEPs adopted by their organization. In total, 27 distinct HFEPs were identified across the five organizations (Table 4.2). Common HFEPs among most organizations were: incorporating choice architecture within food venue layouts, implementing competitive pricing strategies to incentivize healthy purchases, improving the quality of food sourcing, and monitoring sales and procurement data to inform purchasing decisions. Notably, all of the food services were subsidized minimally or substantially by their institution.

\section{Table 4.2 Food Environment Policies Among Organizations}

\begin{tabular}{|c|c|c|c|c|c|}
\hline \multirow[t]{2}{*}{ Healthy Food Environment Policy } & \multicolumn{5}{|c|}{ Organization } \\
\hline & 1 & 2 & 3 & 4 & 5 \\
\hline Antibiotic-free poultry & & & $\mathrm{x}$ & & \\
\hline Choice architecture & & & $\mathrm{x}$ & $\mathrm{x}$ & $\mathrm{X}$ \\
\hline Codified nutritional standards & $\mathrm{x}$ & & $\mathrm{x}$ & & \\
\hline Competitive pricing strategies & & $\mathrm{x}$ & $\mathrm{x}$ & $\mathrm{x}$ & $\mathrm{X}$ \\
\hline Consumer education/education campaigns & & $\mathrm{x}$ & & & $\mathrm{x}$ \\
\hline Decoupling from fast food chain & & & & $\mathrm{x}$ & \\
\hline Employee wellness policy/committee & $\mathrm{x}$ & $\mathrm{x}$ & $\mathrm{x}$ & & \\
\hline Farmer's market & $\mathrm{x}$ & & $\mathrm{x}$ & & \\
\hline Free cooking classes & & $\mathrm{x}$ & & $\mathrm{x}$ & \\
\hline Monthly free salad bar voucher & & & $\mathrm{x}$ & & \\
\hline Healthy meeting policy & $\mathrm{x}$ & & & & \\
\hline Healthy vending policy/standards & & $\mathrm{x}$ & & $\mathrm{x}$ & \\
\hline Hormone-free milk & & & $\mathrm{x}$ & & \\
\hline Improving food quality/sourcing & $\mathrm{x}$ & & $\mathrm{x}$ & $\mathrm{x}$ & $\mathrm{X}$ \\
\hline Developing in-house Grab\&Go bowl & & $\mathrm{x}$ & & & \\
\hline Labeling nutrient content/sticker & & $\mathrm{x}$ & $\mathrm{x}$ & & $\mathrm{x}$ \\
\hline Limits on sodium and trans-fats & $\mathrm{x}$ & $\mathrm{x}$ & & $\mathrm{x}$ & $\mathrm{x}$ \\
\hline Natural foods store & & & $\mathrm{x}$ & & \\
\hline Routinely pilot diet trends & & & & $\mathrm{x}$ & \\
\hline
\end{tabular}




\begin{tabular}{|c|c|c|c|c|c|}
\hline Robust monitoring and evaluation efforts & & & $\mathrm{x}$ & $\mathrm{x}$ & $\mathrm{X}$ \\
\hline Removing fryers from kitchen & & $\mathrm{X}$ & $\mathrm{x}$ & & $\mathrm{x}$ \\
\hline Healthy 5-feet register policy & & & $\mathrm{x}$ & & $\mathrm{x}$ \\
\hline Removing sugar-sweetened beverages & & $\mathrm{x}$ & & & \\
\hline Limiting comfort food specials & & & & & $\mathrm{X}$ \\
\hline Serving size limits & & & & & $\mathrm{x}$ \\
\hline Subsidizing food service operations & $\mathrm{x}$ & $\mathrm{X}$ & $\mathrm{x}$ & $\mathrm{x}$ & $\mathrm{x}$ \\
\hline Sustainability commitment & & & $\mathrm{x}$ & $\mathrm{x}$ & $\mathrm{x}$ \\
\hline Vegetarian kitchen/fare & & & & & $\mathrm{x}$ \\
\hline
\end{tabular}

\section{Facilitators}

Iterative rounds of thematic analysis generated six categories of facilitators cited among respondents. In descending order of code frequency, categories were: institutional commitment (66 references), employee wellness prioritization (46), technical assistance (40), incrementalism (24), external pressures to change the food environment (21), and champion/change agent (19). For institutional commitment, every respondent affirmed the vital role that executive sponsorship and financial assistance played in adopting

\section{HFEPs:}

"If leadership doesn't get onboard, it frustrates the staff. We have to approve the budget, make sure all of that works. But the majority of the effort comes from the bottom-up ... One of things we did was not charge rent to our cafeterias. So, we had to absorb that impact. And we're big enough, so that's not impossible" [2E].

Respondents also underscored the greater purchasing power that accompanied executive sponsorship: "This past year, we even went a step further ... Suffice it to say that resources available for transitions will always have to have a return on investment" [40]. Though Organizations 1, 2 and 4 had executive institutional commitments, the resistant stakeholders in Organization 1 led to fewer changes than in Organizations 2 and 4. 
Employee wellness prioritization was cited twice as much among executive

leaders (31 references) than operational managers across all organizations (15

references):

"Our diabetes numbers are outrageous, what we're spending on this is expensive, we need meaningful solutions. When you look at the employee wellness triangle, with health and wellness, there's only so much the health plans can do that the individual is responsible for themselves. So the focus really became us. And, if we can get our own house in order, what would that look like?" [1E]

Employee wellness committees and policies was an inductive finding during analysis, emerging as a first step in a process of garnering stakeholder support for HFEPs.

References of vision, size, and organizational norms overlapped when discussing employee health:

"Going back to creating a healthier workforce. And supporting wellness, food is one of those areas, in addition to exercise and stress. [Redacted] is different because we have a health plan and a delivery system all together. When you have the health plan at the table and able look at the numbers, we're an integrated system. You can do things when you have all parts at the table, and realize that investment" [2E].

The strong sense of employee wellness was present in both levels of leadership: "That is pretty much the biggest driver of this-not only do we want to help our caregivers be healthier, but we have an investment in our caregivers" [2O].

Most codes for the technical assistance category were predominantly cited at the operational level, with managers emphasizing the need for logistical support, nutritional expertise from dieticians, and organizational resources: "The [consulting group] did some market research for us. We already had the idea of where we were going and they helped reinforce that. And they actually helped us design and develop [redacted], our natural foods store that's up there." [3E] However, tensions rose if logistical support did not 
include the operational members, which was particularly evident when Organization 3 hired six different consulting groups over a period of three years: "I thought about quitting and then telling them what to do! Just kidding. I still need a check.” [3O]

For the incrementalism category, Organizations 3, 4, and 5 had similar views on the value and benefits of building on existing policies and relationships to continually improve actions. Specifically, respondent $4 \mathrm{O}$ discussed at length how his food site was one of 10 that routinely piloted new HFEPs for the remaining 170 interorganizational food venues within his broader network: "We are going to continue offering the Beyond Burger indefinitely. [EW]: What about the sushi pilot, is that coming to a close? [4O]: No, it's successful and definitely staying — it actually increased total sales." Retaining a degree of control over potential outcomes was another desirable feature of incrementalism: "[It] allowed us to make the decisions and see how it was playing out." [3E] Further, the ability to change the food environment gradually, as opposed to a step function, proved to be protective against scaling too quickly: "But we also learned from another site that took out both diet and regular [soda], they ended up bringing back the diet. So we said, OK, we'll start with taking out regular and see where that goes" [2O].

The last two categories, external pressures to change and champion/change agent, had a positive feedback relationship, in that executives cited external pressures and public criticism as impetus for their support in changing the food environments, while operational managers emphasized a need for an executive champion to support them in making changes:

"We knew internally that we were serving slop. Foods were very overcooked and lost taste, nutritional value. For the retail side of it, there aren't any options on 
[redacted], basically, to eat. And so you have a closed system that was not performing very well." [3E]

"When they did the surveying of when the new president came in, he did all the surveying of people of what mattered the most. Food was \#1. I went to my boss and said, 'Are you watching this?' Do you see that this is a really great opportunity for us to like, basically, be like 'Here's what we need?'” [3O]

Of the organizations that most closely overlapped in facilitators cited between managers and leaders, Organization 4 had an overlap of $75 \%$, diverging only on topics of nutritional expertise and the benefit of having a captive audience. Similarly, Organization 5 had an overlap of $68 \%$ between factors cited by both respondents. Of the organizations with the least amount of overlap, Organizations 1 and 2 diverged substantially (44\% and $50 \%$, respectively). Specific facilitators cited by each respondent can be found in Table

\section{3.}

Table 4.3 Respondent-Specific Facilitators of HFEP Implementation

\begin{tabular}{|l|l|l|l|l|l|l|l|l|l|l|}
\hline Facilitator & $\boldsymbol{1 E}$ & $\mathbf{1 O}$ & $\mathbf{2 E}$ & $\mathbf{2 O}$ & $\mathbf{3 E}$ & $\mathbf{3 O}$ & $\mathbf{4 E}$ & $\mathbf{4 O}$ & $\mathbf{5 E}$ & $\mathbf{5 O}$ \\
\hline Champion/change agent & $\mathrm{X}$ & & $\mathrm{X}$ & $\mathrm{X}$ & $\mathrm{X}$ & $\mathrm{X}$ & $\mathrm{X}$ & $\mathrm{X}$ & $\mathrm{X}$ & $\mathrm{X}$ \\
\hline Early adopters & & & & & $\mathrm{X}$ & & $\mathrm{X}$ & $\mathrm{X}$ & $\mathrm{X}$ & \\
\hline Employee wellness prioritization & $\mathrm{X}$ & $\mathrm{X}$ & $\mathrm{X}$ & $\mathrm{X}$ & $\mathrm{X}$ & $\mathrm{X}$ & $\mathrm{X}$ & $\mathrm{X}$ & $\mathrm{X}$ & $\mathrm{X}$ \\
\hline Grant opportunity & $\mathrm{X}$ & & & & & & & & & \\
\hline Open communication & & & $\mathrm{X}$ & & $\mathrm{X}$ & $\mathrm{X}$ & $\mathrm{X}$ & $\mathrm{X}$ & $\mathrm{X}$ & $\mathrm{X}$ \\
\hline Staff buy-in & & & & & & $\mathrm{X}$ & $\mathrm{X}$ & $\mathrm{X}$ & $\mathrm{X}$ & $\mathrm{X}$ \\
\hline Cost savings from employee health & $\mathrm{X}$ & & $\mathrm{X}$ & $\mathrm{X}$ & & & & & & \\
\hline Incrementalism & $\mathrm{X}$ & $\mathrm{X}$ & $\mathrm{X}$ & $\mathrm{X}$ & $\mathrm{X}$ & $\mathrm{X}$ & $\mathrm{X}$ & $\mathrm{X}$ & $\mathrm{X}$ & $\mathrm{X}$ \\
\hline Ability to pilot & & $\mathrm{X}$ & & $\mathrm{X}$ & & $\mathrm{X}$ & $\mathrm{X}$ & $\mathrm{X}$ & $\mathrm{X}$ & $\mathrm{X}$ \\
\hline Institutional commitment & $\mathrm{X}$ & $\mathrm{X}$ & $\mathrm{X}$ & $\mathrm{X}$ & $\mathrm{X}$ & $\mathrm{X}$ & $\mathrm{X}$ & $\mathrm{X}$ & $\mathrm{X}$ & $\mathrm{X}$ \\
\hline Available financial resources & & & $\mathrm{X}$ & $\mathrm{X}$ & $\mathrm{X}$ & $\mathrm{X}$ & $\mathrm{X}$ & $\mathrm{X}$ & $\mathrm{X}$ & $\mathrm{X}$ \\
\hline Competitive pricing strategies & & & & & & & & & & $\mathrm{X}$ \\
\hline Market promotion & & & $\mathrm{X}$ & $\mathrm{X}$ & $\mathrm{X}$ & & & & & \\
\hline $\begin{array}{l}\text { Profit making food operations } \\
\text { units }\end{array}$ & & & & & & $\mathrm{X}$ & & & & \\
\hline Mandate authority & $\mathrm{X}$ & $\mathrm{X}$ & & $\mathrm{X}$ & $\mathrm{X}$ & & $\mathrm{X}$ & $\mathrm{X}$ & $\mathrm{X}$ & $\mathrm{X}$ \\
\hline $\begin{array}{l}\text { High bargaining power with } \\
\text { vendors }\end{array}$ & & & & $\mathrm{X}$ & $\mathrm{X}$ & & $\mathrm{X}$ & $\mathrm{X}$ & $\mathrm{X}$ & \\
\hline
\end{tabular}




\begin{tabular}{|l|l|l|l|l|l|l|l|l|l|l|}
\hline Strong food services mission & & & $\mathrm{X}$ & & $\mathrm{X}$ & & & $\mathrm{X}$ & $\mathbf{X}$ & $\mathrm{X}$ \\
\hline $\begin{array}{l}\text { Subsidizing food service } \\
\text { operations }\end{array}$ & & & $\mathrm{X}$ & & $\mathrm{X}$ & $\mathrm{X}$ & & & $\mathrm{X}$ & $\mathrm{X}$ \\
\hline External pressures to change FE & $\mathrm{X}$ & & $\mathrm{X}$ & $\mathrm{X}$ & $\mathbf{X}$ & $\mathrm{X}$ & $\mathrm{X}$ & $\mathrm{X}$ & $\mathrm{X}$ & $\mathrm{X}$ \\
\hline Captive audience & & & & & $\mathrm{X}$ & & & $\mathrm{X}$ & $\mathrm{X}$ & $\mathrm{X}$ \\
\hline Consumer demand & & & & & $\mathrm{X}$ & $\mathrm{X}$ & $\mathrm{X}$ & $\mathrm{X}$ & $\mathrm{X}$ & \\
\hline Technical assistance & & & $\mathrm{X}$ & $\mathrm{X}$ & $\mathbf{X}$ & $\mathrm{X}$ & $\mathbf{X}$ & $\mathrm{X}$ & $\mathrm{X}$ & $\mathrm{X}$ \\
\hline Available administrative resources & & & $\mathrm{X}$ & & & & $\mathrm{X}$ & & $\mathrm{X}$ & \\
\hline Centralized operations & & & & & & & $\mathrm{X}$ & $\mathrm{X}$ & & \\
\hline Highly skilled kitchen staff/chef & & & & & $\mathrm{X}$ & $\mathrm{X}$ & $\mathrm{X}$ & & $\mathrm{X}$ & $\mathbf{X}$ \\
\hline Nutritional expertise & & & & & $\mathrm{X}$ & $\mathrm{X}$ & $\mathrm{X}$ & & & $\mathrm{X}$ \\
\hline Skills sharing among sites & & & $\mathrm{X}$ & & $\mathrm{X}$ & $\mathrm{X}$ & & & & $\mathrm{X}$ \\
\hline
\end{tabular}

*shaded $\mathrm{X}$ boxes signify more than 5 coded references were made

\section{Barriers}

Similar to the facilitator analysis, the five-stage coding process generated 30 barriers, which were collapsed into five categories: resource constraints (50 references), prescriptive centralization (42), complexity (30), pushback (28), and lack of leadership (19). Resource constraints were uniformly cited by all respondents as the largest barrier to implementing HFEPs, with some of these codes overlapping with an emergent barrier: mutual mistrust. For some respondents, this perceived mistrust was between food service operations and the host institution: "I'd say a barrier is that [redacted] is constantly saying how tough money is. [Chuckles] Especially during negotiations, right?" [3E]. "Isn't it ironic? That the [redacted] was costing us $\$ 100 \mathrm{~K}$ to operate and they closed it, only to put [redacted] here and it costs us more than $\$ 100 \mathrm{~K}$ between utilities and loss of sales" [5O]. For others, it was between the operations staff and executives: "I had that thought today, honestly, "Did [redacted] just hire me so that they could say we have a sustainability manager and we 're doing good things?" [3O]. 
One executive explained that the large resource constraints meant that food service staff were not paid competitive wages, leading to "high turnover and vacancy rate, [which] means we might not be able to have every single station open, we might have fewer cashiers, so there's waits... and so that's a more contextual barrier" [4E]. For some, the resource scarcity added pressure to make up the funds elsewhere: "Yes, there would be institutional pressure to make changes. We have done that. We have laid people off and reduced positions. We needed to downsize our catering group by a third, so a lot of people left the organization" [3E].

In addition to the resource constraints, prescriptive centralization stood as a topdown barrier for organizations who wanted to innovate within their onsite food environments:

"Interestingly, we have prohibitions in the federal government from advertising. We can educate but it's set up to not compete with the private sector. So we can educate about the [redacted], but we can't post them in the lobby because we can't advertise. So, for the marketing question you have, it's a little tricky to get the customers into the canteen but once they do, we have placards about healthy choices ... but if they're posted around the hospital, we take them down." [4E]

Similarly, after respondent [1E] concluded that her current vendor was not meeting the needs of the building tenants, she was surprised to realize that she couldn't competitively solicit bids for another vendor: "I don't know if you've dug into the actual rules around the mini Randolph Sheppard Act in Oregon, because technically written into law is that the licensed vendors and the [redacted] have right of first refusal, which means they have priority over all of our state-contracted concessions." While these institution-wide prohibitions or legislated stakeholders represented structural barriers, process-oriented 
barriers emerged in the form of pushback. When HFEPs were implemented, nearly every interview respondent said that there was some level of pushback, no matter how minor. For some HFEPs, consumers asked "why they didn't go far enough" [5O]. However, the more frequent response to a HFEP was some version of liberty infringement: "Why are you telling me what to do, I'll make that choice myself. When [redacted] took out their fryers, you would think that we extracted every first male born child" (sic) [2E]. Both operational and executive leaders cited this factor as a consistent barrier.

Complexity was also cited by both levels of leadership, typically in relation to untangling bureaucratic situations:

"So yes, we'd be one of very few—I'm trying to think—so, [redacted; "Agency"] is the agency that is - they're basically our landlord for most of our facilities. So [redacted; "Division"], we rent this space from [Agency]. So technically, the cafeteria downstairs--that's not in operation right now-[Agency] contracts with the [Vendor], and the [Vendor] then contracts with [Distributor]'s licensed vendors to operate the facilities" [1E].

For the last category, lack of leadership, this subset of codes was predominantly cited among operation-level leaders (three times more than executive). Lack of leadership subsumed codes such as unclear goals and goal conflicts, and operation managers levied these failures against their executive counterparts:

"It used to be that all the supervisors and management would sit a room together every week and we would have conversations. That hasn't happened in years. They just stopped. They just got tired of all the infighting and just stopped all of it. Because all the different locations are managed by different people. And they all can do whatever the hell they want. So there's no consistency." [3O]

Of the organizations that most closely overlapped in barriers cited between managers and leaders, Organization 1 had an overlap of $58 \%$, diverging in perspectives 
on unclear messaging, lack of leadership/mandate authority, and consumer preferences.

Similarly, Organization 5 had an overlap of $55 \%$ between factors cited by both

respondents. Of the organizations with the least amount of overlap, Organization 4

diverged significantly among respondent perspectives, aligning only $33 \%$ of the time.

Organizations 2 and 3 had a similar degree of overlap ( $40 \%$ and $44 \%$, respectively)

(Table 4.4).

Table 4.4 Respondent-Specific Barriers to HFEP Implementation

\begin{tabular}{|l|l|l|l|l|l|l|l|l|l|l|}
\hline Barrier & $\boldsymbol{1 E}$ & $\mathbf{1 O}$ & $\mathbf{2 E}$ & $\mathbf{2 O}$ & $\mathbf{3 E}$ & $\mathbf{3 O}$ & $\mathbf{4 E}$ & $\mathbf{4 O}$ & $\mathbf{5 E}$ & $\mathbf{5 O}$ \\
\hline Complexity & $\mathrm{X}$ & $\mathrm{X}$ & & & $\mathbf{X}$ & $\mathrm{X}$ & $\mathrm{X}$ & $\mathrm{X}$ & $\mathrm{X}$ & $\mathrm{X}$ \\
\hline Unclear goals & $\mathrm{X}$ & & & & $\mathrm{X}$ & $\mathrm{X}$ & $\mathrm{X}$ & $\mathrm{X}$ & $\mathrm{X}$ & \\
\hline Inconsistent messaging & $\mathrm{X}$ & & & & & $\mathrm{X}$ & $\mathrm{X}$ & & $\mathrm{X}$ & $\mathrm{X}$ \\
\hline Lack of leadership & & $\mathrm{X}$ & & & $\mathrm{X}$ & $\mathrm{X}$ & & $\mathrm{X}$ & $\mathrm{X}$ & \\
\hline Perceived goal conflict & & & & & $\mathrm{X}$ & & & $\mathrm{X}$ & $\mathrm{X}$ & $\mathrm{X}$ \\
\hline Prescriptive centralization & $\mathrm{X}$ & $\mathbf{X}$ & & $\mathrm{X}$ & $\mathbf{X}$ & $\mathrm{X}$ & $\mathrm{X}$ & & $\mathrm{X}$ & $\mathrm{X}$ \\
\hline Institutional bias & $\mathrm{X}$ & $\mathrm{X}$ & & & & & & & & $\mathrm{X}$ \\
\hline Long-term vendor contracts & & & & & & & & & & $\mathrm{X}$ \\
\hline Power struggle & & & & $\mathrm{X}$ & $\mathrm{X}$ & $\mathrm{X}$ & & & $\mathrm{X}$ & $\mathrm{X}$ \\
\hline Mutual mistrust & & & & & $\mathrm{X}$ & $\mathrm{X}$ & & & & $\mathrm{X}$ \\
\hline Prohibitions on marketing & & & & & & & $\mathrm{X}$ & $\mathrm{X}$ & & \\
\hline Statutory barriers & $\mathrm{X}$ & $\mathrm{X}$ & & & & $\mathrm{X}$ & & & & \\
\hline Lack of mandate authority & & $\mathrm{X}$ & & & & $\mathrm{X}$ & & & & \\
\hline Pushback & $\mathrm{X}$ & $\mathrm{X}$ & $\mathrm{X}$ & $\mathrm{X}$ & $\mathrm{X}$ & $\mathrm{X}$ & $\mathrm{X}$ & $\mathrm{X}$ & $\mathrm{X}$ & $\mathrm{X}$ \\
\hline $\begin{array}{l}\text { Competition with surrounding } \\
\text { vendors }\end{array}$ & & & & & $\mathrm{X}$ & & & $\mathrm{X}$ & & $\mathrm{X}$ \\
\hline $\begin{array}{l}\text { Consumer resistance/preference for } \\
\text { unhealthy items }\end{array}$ & $\mathrm{X}$ & & $\mathrm{X}$ & & & & & $\mathrm{X}$ & $\mathrm{X}$ & $\mathrm{X}$ \\
\hline Disgruntled staff/lack of buy-in & & & & $\mathrm{X}$ & & $\mathrm{X}$ & $\mathrm{X}$ & & $\mathrm{X}$ & \\
\hline Lack of competent/skilled staff & & & & & & & $\mathrm{X}$ & & & \\
\hline Disgruntled vendors & $\mathrm{X}$ & $\mathrm{X}$ & $\mathrm{X}$ & $\mathrm{X}$ & $\mathrm{X}$ & & & & & \\
\hline High stress environment & & & $\mathrm{X}$ & & & & & $\mathrm{X}$ & & \\
\hline Resource constraints & $\mathrm{X}$ & $\mathrm{X}$ & $\mathrm{X}$ & $\mathrm{X}$ & $\mathrm{X}$ & $\mathrm{X}$ & $\mathrm{X}$ & $\mathrm{X}$ & $\mathrm{X}$ & $\mathrm{X}$ \\
\hline Actual revenue loss & & & $\mathrm{X}$ & $\mathrm{X}$ & $\mathrm{X}$ & & & & $\mathrm{X}$ & $\mathrm{X}$ \\
\hline Perceived revenue loss & & & & $\mathrm{X}$ & $\mathrm{X}$ & & & & $\mathrm{X}$ & $\mathrm{X}$ \\
\hline Higher food costs & & & & & & $\mathrm{X}$ & & $\mathrm{X}$ & & $\mathrm{X}$ \\
\hline Higher labor costs & & & $\mathrm{X}$ & $\mathrm{X}$ & & $\mathrm{X}$ & & $\mathrm{X}$ & $\mathrm{X}$ & $\mathrm{X}$ \\
\hline
\end{tabular}




\begin{tabular}{|l|l|l|l|l|l|l|l|l|l|l|}
\hline Lack of money & X & X & & & X & X & & & & X \\
\hline Lack of training or capacity & X & X & & X & X & X & X & & X & \\
\hline No monitoring possible & X & X & & & & X & & & & \\
\hline Staff turnover & & & & & & X & X & & X & \\
\hline
\end{tabular}

*shaded $\mathrm{X}$ boxes mean the code had at least 5 references

\section{Discussion}

Among this purposive sample of five health care organizations and 10 interview respondents, we identified 30 barriers to and 27 facilitators of HFEP implementation. From these, we developed overarching implementation factors that were consistently cited by respondents across all of the participating organizations. As expected, complementary leadership types cited factors corresponding to their domain, as executive perceptions of HFEP implementation focused on issues such as stakeholder management or external pressures whereas operational perceptions focused on logistical aspects of HFEP, such as technical assistance and nutritional expertise. However, where these two realms typically conflicted was when executives over-delegated challenges to their operational counterparts, as was the case with Organizations 3 and 5. In the organizational literature, delegation spoke to a leadership style whereupon executives were trained to stay out of the minutiae of the day-to-day, relying on junior members to respond dynamically to challenges (Klein et al, 2006; Zyngier, 2013). However, when it came to intractable problems that can only be addressed with executive approval, this delegatory leadership style oversimplified and underestimated the scope of HFEP implementation barriers, thereby frustrating organizational operators. The additional 
finding of mutual mistrust among some respondents (3O and 5O) aggravated the implementation challenges.

Another critical implementation factor was the universally cited resource constraint. The inability to purchase technology, kitchen equipment, or proprietary materials to implement a HFEP led one operational manager to get creative about cutting overhead costs: "We eliminate[d] the need for us to carry a labor pool. It's their labor pool and we just contract with them. We get a fixed amount from their sales, and in return, we have no cost of goods and no cost of labor" [40]. The decision to contract with a vendor for their labor pool seemed to be a risk-free proposition to the manager. However, the decision to structurally realign to an outside vendor's labor supply can have long-term repercussions of vendor reliance, inability to decouple, and ultimate dependence.

Finally, merging the overarching categories of facilitators and barriers into three complementary recommendations can be a useful heuristic to overcome HFEP implementation challenges. Just as operational and executive leaders are complementary in role, viewing the barrier and facilitator subgroups as reciprocal groups can help to map out tangible next steps:

1. Address the 'Lack of leadership' category with the corresponding 'Champion/change agent' category to fill the leadership void with a goal-oriented advocate.

Persistent challenges without a leader who continuously problem-solves can lead to a construct in the organizational literature known as 'inertia' (Hannan \& Freeman, 1984; Coiera, 2011). Fairholm (2009) describes the critical role of leaders as agenda-setters: 
determining objectives, setting priorities and anticipating potential opportunities. The positive feedback relationship found between 'external pressures' and 'change agent' categories was cited among both operational and executive leaders. This suggests that, as public criticism grows for healthier food environments, change agents will emerge to champion the need to improve retail venues.

2. Solve the 'Complexity' barrier category with 'Technical assistance' facilitators. Some HFEP challenges appear ambiguous or multifactorial, which leads to an unclear goal or "pulls and pushes" (Hannan \& Freeman, 1993). Technical assistance strategies such as availing nutritional expertise from onsite dietetics or resources from participating collaboratives could transform obstacles that are seemingly insurmountable into problems that can be managed under the operational manager's purview. However, operational managers perspectives should be included when considering outside consultation, as failing to do so (e.g., top-down decisions) was shown to increase tension among levels of leadership (Organization 3), later contributing to an overall sense of mistrust among leaders.

3. Overcome 'Resource constraints' with the 'Institutional commitment' category. As mentioned in the introduction, food service operations are unlike inpatient dietary settings in a number of ways, most significantly in that they have a budget that introduces the element of needing to vend food and beverage options that will entice purchase and appeal to mass tastes. When and if a HFEP under consideration will lead to a reduction in overall sales (irrespective of whether this barrier is perceived or actual), institutional commitment strategies of financial subsidies, institutional bargaining power and public 
organization-wide support of HFEP development can be necessary to advance HFEP goals, as was the case in each of the five organizations in this study.

\section{Limitations}

Our study has several limitations. First, enrollment of study organizations was restricted to one state, thereby limiting the transferability of findings to out-of-state organizations that have different policy contexts. Oregon is fairly progressive politically as well as civically, thus consumer demand, public perception, organizational norms, and health care leaders' opportunities might vary sufficiently so as to pose novel implementation challenges not captured in this study. Second, the single coder approach is a significant limitation given that this work is part of a broader dissertation study. However, this was largely mitigated by active dissertation committee oversight, with one committee member (RB) having specific expertise as to the methods and analytic techniques.

Notwithstanding these limitations, this study provides insights to intra- and interorganizational factors surrounding HFEP implementation.

\section{Conclusion}

This study examined HFEP implementation within five health care organizations, providing insight on the challenges of accurately measuring leaders' perceptions of the facilitators and barriers to this process. Overlap of factors cited between both levels ranged from $44-75 \%$, such that paying attention to the perspectives of these two distinct levels of leadership can be useful when determining strategies for overcoming implementation challenges. Finally, as leaders look to the academic literature to prepare 
for HFEP implementation, understanding the common barriers and challenges and identifying corresponding facilitators can help leaders, particularly operational managers, to prepare a plan of action if and when implementation obstacles arise. 


\section{CHAPTER 5: AN INTERRUPTED TIME-SERIES ANALYSIS TO EVALUATE A HEALTH SYSTEM'S SUGAR-SWEETENED BEVERAGE SALES BAN: OUTCOMES ON REVENUE AND CONSUMER BEHAVIOR}

\section{Introduction}

The rate of diet-related chronic diseases has increased in the US every decade since 1960 (Fryar et al, 2018). Among many factors, a driver of this public health crisis (Seidell et al, 2015) is obesogenic food environments, characterized by nutrient-poor and energy-dense foods that saturate the collective physical, economic and sociocultural conditions, which in turn influences nutritional status (Townsend \& Lake, 2017; Hall et al, 2018, Swinburn et al, 2015). Over the past 30 years, sugar-sweetened beverages (SSBs), which include regular (non-diet) soda, lemonades, and fruit drinks, have emerged as one particularly concerning contributor to obesogenic food environments, with non-diet soda accounting for $46 \%$ of added calories in the average U.S. diet while providing no nutritional value (Campos, 2011; Malik et al, 2006). Since most Americans consume about one-third of their calories away from the home (Viera et al, 2019), obesogenic food environments can promote the consumption of SSBs by making them more affordable, accessible, and convenient.

Given the considerable time employees spend in the workplace, coupled with the fact that most workers in the US obtain health insurance through their jobs (Abramowitz \& O’Hara, 2017), employers have increasingly taken notice of worksite food environments, their impact on food choice, and long-term employee health (Basu et al, 
2020). Employers in nearly every sector have begun implementing nutritional initiatives to foster healthier food environments and decrease their health spending on premiums and illness (Gardner et al, 2014; Symonds et al, 2013; Mhurchu et al, 2010). Notable examples include employers such as Google, Nike, Microsoft, Twitter, and WeWork, all of whom have implemented large-scale nutritional changes to foster improvement of dietary behaviors among their employees (McGregor, 2018; Barberio, 2017; Lev-Ram, 2015; Nestle, 2011).

Workplace nutritional initiatives range in scope and effectiveness (Schliemann \& Woodside, 2019; Nathan et al, 2020). The spectrum of practices includes minimal adjustments, such as placing unhealthy options in less convenient areas, to comprehensive multicomponent interventions (Timmer et al, 2018; Kimmons, 2012; Sorenson et al, 2001). In their examination of 114 worksite interventions focused on improving worksite physical and food environments, Pratt and colleagues (2007) conclude that the more comprehensive the worksite intervention (e.g., large-scale structural changes in addition to addressing individual behaviors), the greater the improvement in employee health outcomes. In addition to demonstrating effectiveness of a policy on employee health outcomes (Epel et al, 2020), organizational leaders must also factor in public perception and implementation costs when determining which policies to adopt. Moreover, employers considering nutritional initiatives must contend with the immediate and long-term economic sustainability of prospective policies (Lerner et al, 2013; Kaspin et al, 2013; Proper \& van Mechelen, 2008).

Absent a galvanizing commitment to a large-scale overhaul, employers will often choose minimally disruptive practices to implement within their organizations (e.g., those 135 
with high public acceptance and low resource requirements), despite evidence that these conservative approaches may produce a smaller health impact than comprehensive approaches (Pratt et al, 2007). Often labeled as incremental approaches, conservative workplace practices can include reducing serving sizes (Hollands et al, 2018), incorporating choice architecture $^{2}$ within layouts of onsite cafeterias (Geaney et al, 2016; Thorndike et al, 2014), implementing stoplight labeling (Gardner et al, 2014), or keeping unhealthy products at least five feet away from the check-out registers (Fernandes et al, 2016). The literature indicates that fear of revenue loss is the largest barrier to implementing comprehensive food environment policies (Mozaffarian et al, 2018; Park \& Lee, 2016; Cohen et al, 2016; Bell, 2013; Wharton, Olstad, 2012; Long \& Schwartz, 2008). In their systematic review of worksite dietary interventions, Mhurchu and colleagues (2010) underscore that cost-effectiveness should be a prioritized metric when evaluating worksite health programs, as employers often overestimate the resource costs needed to implement such interventions. Therefore, establishing the economic feasibility of a workplace intervention can be just as important as demonstrating the population health benefit.

Among the interventions available to improve the food environment, removing SSBs represents an effective practice for improving population health (Basu et al, 2020; Eneli et al, 2014; Moran et al, 2015). Recent research evaluating a workplace SSB ban found a $48.5 \%$ average reduction in SSB consumption and significantly less belly fat among the 202 employee-participants (Epel et al, 2020). While the health improvements

\footnotetext{
${ }^{2}$ The practice of making healthy foods more physically accessible, visible and priced competitively while moving unhealthy foods to harder-to-access areas in a food venue layout.
} 
have been demonstrated (Taillie et al, 2020; Basu et al, 2020; Van Duong, 2020;

Mekonnen et al, 2013), the consequence of SSB bans on revenue is not clear (Pechey et al, 2019; Yan et al, 2017; Jilcott Pitts et al, 2016; Cradock et al, 2015; Grech \& Allman, 2015; Olstad, 2013; Vander Wekken et al, 2012). This may be because most studies examining consumer or revenue effects resulting from SSB bans typically report shortterm outcomes, which hinders the ability to determine if revenue trends have latent effects such as rebounding from an initial decrease or stabilizing over time (Eneli, 2014; Taber et al, 2012; Epel et al, 2020). Additionally, many studies that evaluate either health or revenue outcomes of SSB bans do not examine consumer purchasing changes (i.e., types of beverages that increased in demand post-SSB ban), which could inform the sociocultural changes associated with SSB bans. Thus, studying the medium- and longterm trajectories of net revenue and consumer behaviors may be helpful for employers that are contemplating the removal of SSBs from their worksites.

The objective of our study was to determine the long-term fiscal impact of removing SSBs from the workplaces of a large health system with over a dozen clinics and hospitals throughout Oregon. The health system, which employs nearly 21,000 workers and has over 4 million visits annually, implemented a ban on SSBs in January 2015 as part of a broader nutritional initiative. The SSB ban included regular soda but the health system opted to retain diet sodas, juices and sports/energy drinks as part of its incremental strategy to improve the worksite food environment.

\section{Methods}

Data Source 
We examined monthly point-of-sale revenue reports from all onsite retail food venues within a large health system from January 2012 to November 2019 (95 months). Sales reports three years before and four years after the SSB ban were obtained from the health system. Data obtained contain product names, sales counts, sales totals, venue names, and dates of sale. Further, the point-of-sales data contain characteristic information of the food venues, such as if it is a cafeteria, café or coffee cart as well as the geographic location.

\section{Analytic Sample}

Beverage-specific revenue was determined and categorized by beverage type. Because of the way the data were labeled in the point-of-sales system, it was not possible to differentiate between diet and regular soda in the pre-ban period. Therefore, both diet and regular soda are in one category (labeled 'all sodas'), with other beverage categories being juice, water, tea, coffee, or sports/energy drinks. Retail food venues with complete reporting for all 95 months were included in the dataset. Out of 19 food venues within the health system, two venues with incomplete sales reporting were excluded—one venue had 83 months available and one venue had 71 months available. The unit of analysis is site-month, with a total of 1615 site-months available.

\section{Study Design}

We employed an interrupted time-series (ITS) design for this study. First, the time series was split into pre-intervention and post-intervention phases, with January 2015-the month the nutritional initiative was implemented - as the "interruption." The first three 
months of 2015 were excluded from the analysis to account for complete implementation and the sales receipt lag. There are 36 months prior to the initiative and 56 months following the implementation period. The primary outcome was change in gross beverage sales after implementation of the SSB ban compared to the trend before the ban (counterfactual condition). Covariates include sales by venue type (coffee cart, café, cafeteria), venue size based on FY 2012 gross revenue (small, $\leq \$ 50,000$; medium, $\leq \$ 100,000$; large, $>\$ 100,000$ ), and venue location (rural, suburban or urban). Secondary outcomes were percent change in sales by beverage type before and after the ban (water, coffee, tea, sports/energy drinks, milk, all sodas); and percent change in sales by venue type, size and location. This study was considered exempt from institutional review by Portland State University (Study \#196741-18).

\section{Statistical Analysis}

A piecewise linear regression model was used to assess the effect of the SSB ban on gross beverage sales. Given the time series nature of the data, ordinary least-squares estimation was employed to test for homogeneity of variance and autocorrelation. Levene's test revealed no significant departure for either pre/post groups or year groups, indicating that the use of least-squares estimation was appropriate for this analysis. We calculated the Durbin-Watson statistic to test for within-worksite single-lag autocorrelation of the error terms in the regression model. Autocorrelation was not detected $(2,95=1.93)$, with the d-statistic close to the center of distribution of 2.0. Next, we tested for non-stationarity of the data using the Kwiatkowski-Phillips-Schmidt-Shin (KPSS) test, and tested for stationarity using the augmented Dickey-Fuller (ADF) test. 
We failed to reject the null for KPSS (for nine lags) and we rejected the null for ADF $(\mathrm{P}>.0000)$, with both tests indicating data stationarity. We used the Wilcoxon-MannWhitney rank sum test, a nonparametric test, to evaluate whether the revenues were different between the pre- and post-ban periods. All analyses were performed using Stata version 16.1 (Statacorp, 2019). A p-value less than .05 was considered statistically significant.

\section{Sensitivity Analyses}

To test the robustness of the main findings, we included all 19 food venues in the rank sum test and found the model results were not different from our main findings (71 months, $\mathrm{n}=1349$ site-months). We also created three alternate cutoff points for our analysis: 6 months, 12 months, and 18 months following implementation of the SSB ban.

\section{Results}

\section{Baseline Characteristics}

Our main analysis included 17 food venues with complete sales data from five urban, 10 suburban and two rural food venues. The majority were cafeterias (10), with the remaining being cafés (4) or coffee-carts (3). There were nearly equal groups of small, medium and large food venues (Table 5.1). 
Table 5.1 Baseline Characteristics of 17 Food Venues Pre-SSB Ban, Jan 2012-Dec 2014

\begin{tabular}{|l|c|c|c|}
\hline & $\begin{array}{c}\text { Number of } \\
\text { food venues, } \mathrm{n} \\
(\%)\end{array}$ & $\begin{array}{c}\text { Mean monthly revenue pre-SSB } \\
\text { ban } \\
\text { (Jan 2012-Dec 2014) }\end{array}$ & $\begin{array}{c}95 \% \text { confidence } \\
\text { intervals (in dollars) }\end{array}$ \\
\hline Systemwide (Total) & $17(100)$ & $\$ 273,570$ & $269,180-277,960$ \\
\hline By Location & \multicolumn{3}{|c|}{} \\
\hline Urban & $5(30)$ & $\$ 127,319$ & $124,474-130,164$ \\
\hline Suburban & $10(58)$ & $\$ 138,508$ & $135,622-141,394$ \\
\hline Rural & $2(12)$ & $\$ 7,742$ & $7,563-7,920$ \\
\hline By Type & $10(58)$ & $\$ 132,307$ & $129,808-134,806$ \\
\hline Cafeteria & $4(24)$ & $\$ 101,762$ & $100,071-103,453$ \\
\hline Café & $3(18)$ & $\$ 39,500$ & $38,437-40,563$ \\
\hline Coffee cart & $6(35)$ & $\$ 205,642$ & $202,152-209,132$ \\
\hline By Size $(\mathrm{FY} 2012$ revenue) & $5(30)$ & $\$ 51,332$ & $50,314-52,350$ \\
\hline Large $(>\$ 100,000)$ & $6(35)$ & $\$ 16,595$ & $16,238-16,952$ \\
\hline Medium $(<\$ 100,000)$ &
\end{tabular}

After the introduction of the SSB ban, the mean monthly beverage revenue decreased from $\$ 273,570$ to $\$ 256,110$, representing a $6.38 \%$ decrease in beverage sales across the entire health system (Table 5.2). The quarterly percent changes in sales varied widely throughout the study time period, with a low of $-8.04 \%$ in first quarter of 2017 and a high of $4.04 \%$ in the fourth quarter of 2018 (Figure 5.1). These wide-ranging percent changes continue to fluctuate throughout the end of the study time period. 
Figure 5.1 Monthly Gross Beverage Revenue, Jan 2012-Nov 2019, Inclusive

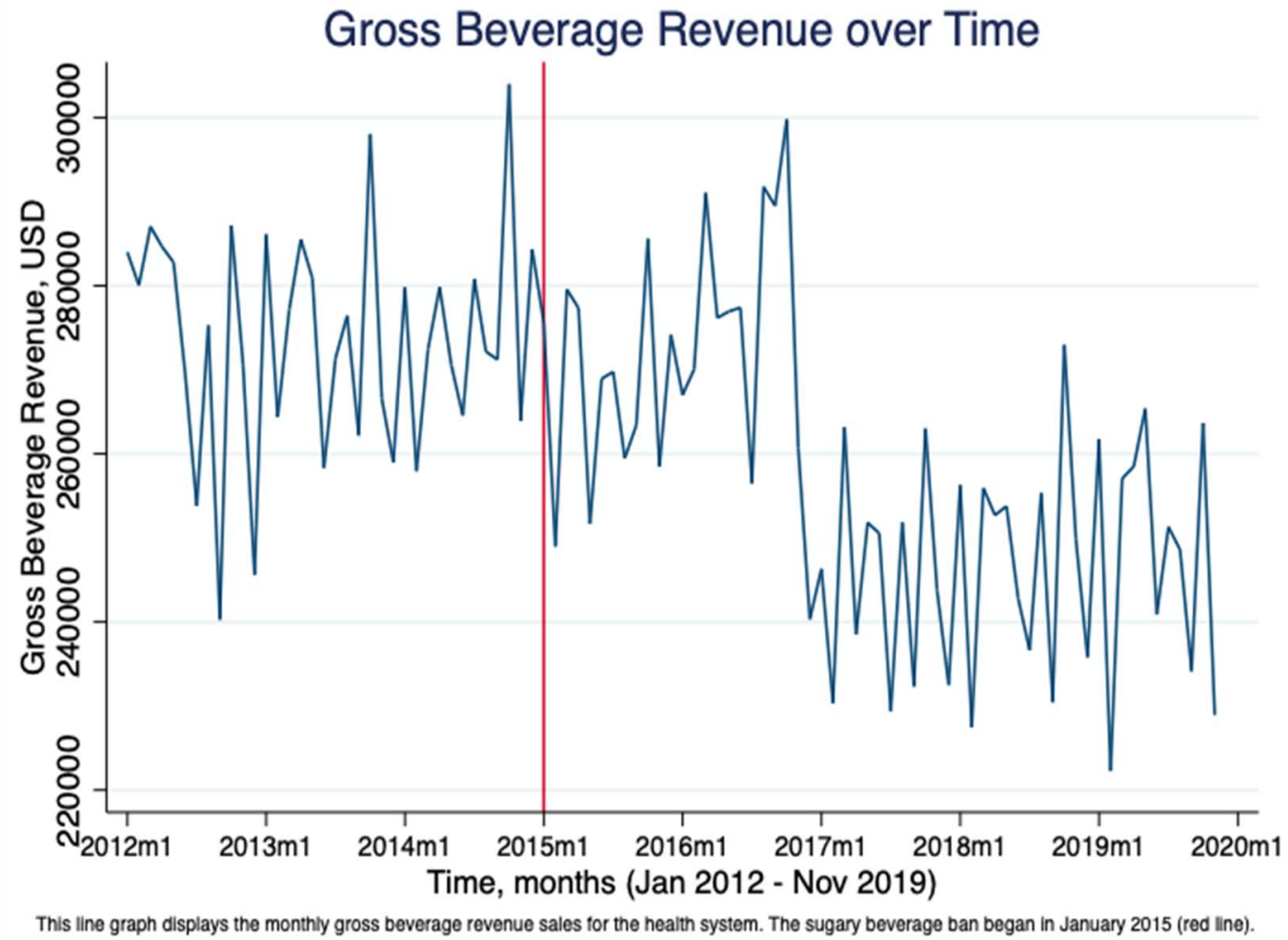

\section{Gross Beverage Sales}

For gross beverage revenue, the Wilcoxon-Mann-Whitney U test showed that the fouryear period following the SSB sales ban was statistically significantly different (reduced) from the pre-ban time period $(\mathrm{p}<.0001)$. When restricting the post-ban study period to 6 , 12, and 18 months, revenues were not decreased from the pre-implementation period. This can be visually confirmed in Figure 5.1, where there was no immediate decrease in revenue after implementation of the SSB ban and for almost two years afterward, followed by a sharp level decrease in sales beginning in late 2016. Figure 5.2 displays the 
pre-ban fitted line, post-ban fitted line, as well as the counterfactual fitted line to indicate the trend absent the SSB ban.

Figure 5.2 Fitted Lines for Gross Monthly Revenues in the Health System, Pre and Post SSB Ban, Inclusive

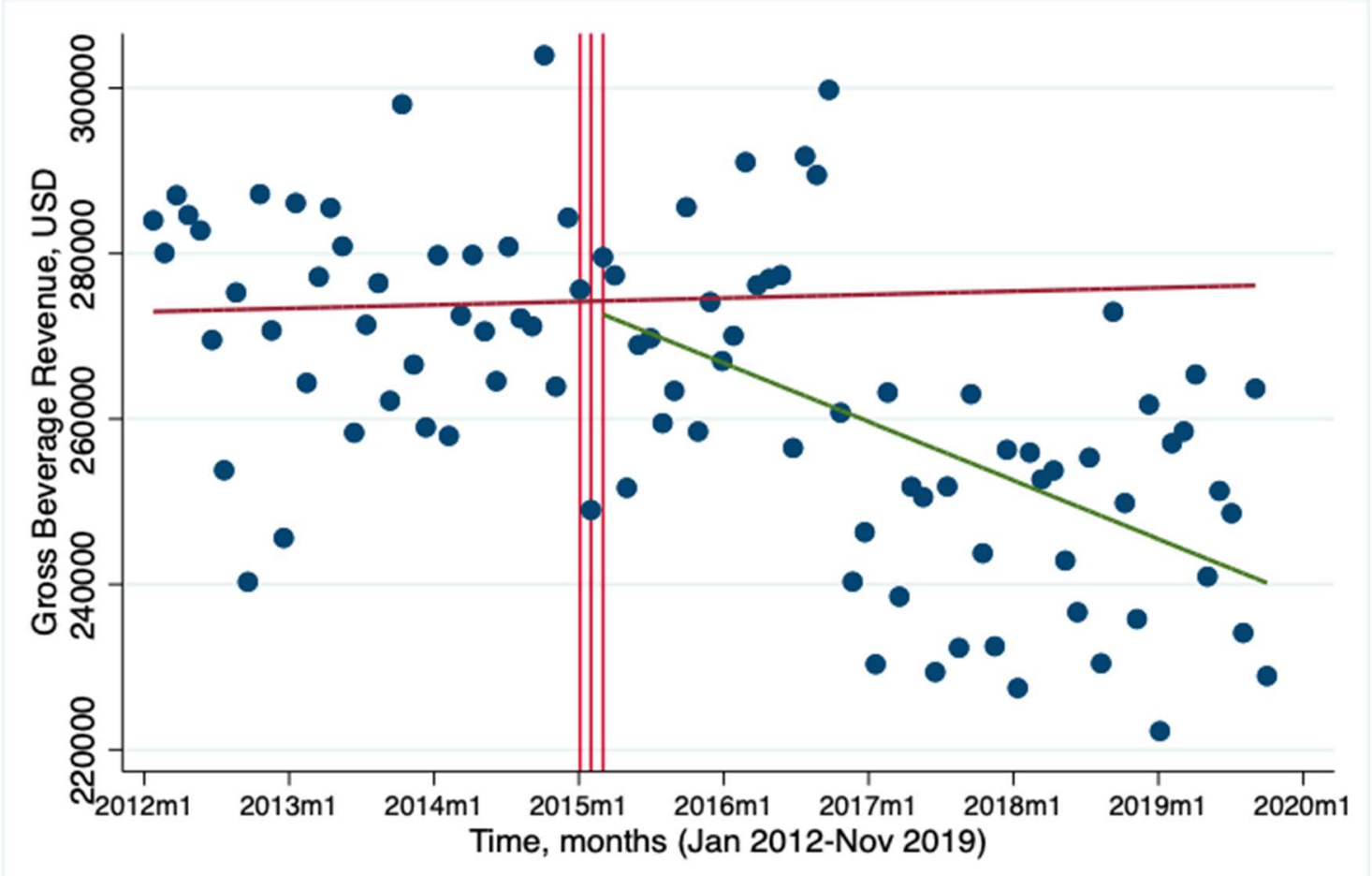

- Sales value — Fitted line with no ban — Fitted line after ban

\section{Beverages Sales by Type}

In the years leading up to the SSB ban, coffee sales were the highest grossing beverage category $(\$ 134,974)$ per month, followed by all sodas $(\$ 52,949)$ and juice $(\$ 28,747)$ sales. After implementation of the ban, though diet sodas continued to be offered in lieu of regular soda, venues experienced a sharp decrease (-44.7\%) in all soda sales in the 
post-ban time period. After the removal of regular sodas, increases in water $(+35.14 \%)$, sports/energy drinks $(+2.03 \%)$, juice $(+7.45 \%)$ and coffee $(+1.84 \%)$ sales were observed, though only water and juice sale increases were statistically significant (Table 5.2).

Table 5.2 Percent Change in Gross Sales Among Beverage Categories, Pre and Post SSB Ban

\begin{tabular}{|c|c|c|c|c|c|}
\hline \multirow[t]{2}{*}{ Beverage type } & \multicolumn{2}{|c|}{$\begin{array}{c}\text { Mean monthly } \\
\text { revenue, } \$\end{array}$} & \multirow[t]{2}{*}{$\begin{array}{c}\text { Percent } \\
\text { change, \% }\end{array}$} & \multirow{2}{*}{$\begin{array}{c}\text { Percent } \\
\text { change 95\% } \\
\text { CI }\end{array}$} & \multirow[t]{2}{*}{ P-value* } \\
\hline & Pre-ban & Post-ban & & & \\
\hline $\begin{array}{l}\text { All sodas (diet and } \\
\text { regular) }\end{array}$ & 52,949 & $29,277^{\wedge}$ & -44.71 & $-42.96,-46.46$ & 0.0000 \\
\hline Coffee & 134,974 & 137,452 & +1.84 & $-1.28,4.96$ & 0.5637 \\
\hline Tea & 18,313 & 14,421 & -21.25 & $-19.32,-23.18$ & 0.0000 \\
\hline Water & 17,667 & 23,875 & +35.14 & $29.03,41.25$ & 0.0000 \\
\hline Sports/Energy & 9,355 & 9,545 & +2.03 & $-6.16,10.22$ & 0.8829 \\
\hline Juice & 28,747 & 30,888 & +7.45 & $4.12,10.78$ & 0.0002 \\
\hline Milk & 11,564 & 10,651 & -7.90 & $-4.12,-11.68$ & 0.0020 \\
\hline All beverages & 273,570 & 256,110 & -6.38 & $-4.44,-8.32$ & 0.0001 \\
\hline
\end{tabular}

${ }^{\wedge}$ Diet soda continued to be offered in lieu of regular soda.

*P-values were obtained from Wilcoxon-Mann-Whitney U tests.

\section{Covariates: Size, Location, Type}

Differential revenue impacts were observed based on food venue size, geographic location, and type of venue (e.g. cafeteria versus coffee cart). Rural and urban venues experienced statistically significant mean monthly decreases after the ban, $-9.61 \%$ and $14.95 \%$, respectively (Table 5.3). Suburban venues experienced slight increases in revenue in the post-ban period, though this increase was not statistically significant. When stratifying venues by size, both small and large venues observed significant losses in the post-ban period of $-3.74 \%$ and $-9.62 \%$, respectively. Medium-sized venues, primarily located within suburban and urban locations, observed statistically significant increases in mean monthly revenue after the SSB ban. Likewise, all three types of food 
venues experienced significant changes between the pre- and post-ban time periods, with observed decreases in sales among cafés and cafeterias while coffee carts throughout the health system observed sales increases.

Table 5.3 Stratified Revenue Differences, by Covariate, Pre and Post SSB Ban

\begin{tabular}{|c|c|c|c|c|c|}
\hline \multirow[t]{2}{*}{ Covariate } & \multicolumn{2}{|c|}{$\begin{array}{c}\text { Mean monthly revenue, } \\
\$\end{array}$} & \multirow[t]{2}{*}{$\begin{array}{c}\text { Percent } \\
\text { change, } \%\end{array}$} & \multirow{2}{*}{$\begin{array}{c}\text { Percent } \\
\text { change } 95 \% \\
\text { CIs }\end{array}$} & \multirow[t]{2}{*}{ P-value } \\
\hline & Pre-ban & Post-ban & & & \\
\hline \multicolumn{6}{|l|}{ Location } \\
\hline Urban & 127,319 & 108,286 & -14.95 & $-11.36,-18.54$ & 0.0000 \\
\hline Suburban & 138,508 & 140,825 & +1.67 & $-0.44,3.78$ & 0.1240 \\
\hline Rural & 7,742 & 6,998 & -9.61 & $-4.58,-14.64$ & 0.0002 \\
\hline \multicolumn{6}{|l|}{ Size } \\
\hline Large & 205,642 & 185,864 & -9.62 & $-7.32,-11.92$ & 0.0000 \\
\hline Medium & 51,332 & 54,272 & +5.73 & $3.35,8.11$ & 0.0002 \\
\hline Small & 16,595 & 15,974 & -3.74 & $-0.01,-7.47$ & 0.0093 \\
\hline \multicolumn{6}{|l|}{ Type } \\
\hline Cafeteria & 132,307 & 118,752 & -10.25 & $-7.86,-12.64$ & 0.0000 \\
\hline Cafe & 101,762 & 95,226 & -6.42 & $-4.47,-8.37$ & 0.0002 \\
\hline $\begin{array}{l}\text { Coffee } \\
\text { Cart }\end{array}$ & 39,500 & 42,132 & +6.66 & $3.62,9.70$ & 0.0002 \\
\hline
\end{tabular}

Sensitivity Analyses Results

Upon including all 19 food venues (71 months, $\mathrm{n}=1349$ site-months) in the rank sum test, we found the model results were not different from our main findings (data not shown). The three alternate cutoff points of 6 months, 12 months, and 18 months following implementation of the SSB ban revealed no statistically significant differences between the pre- and post-ban periods ( $\mathrm{p}<0.836, \mathrm{p}<0.823$, and $\mathrm{p}<0.128$, respectively).

\section{Discussion}


Among this health system's 17 food venues, the implementation of an SSB ban resulted in no immediate revenue impact on gross beverage sales at 6,12 , and 18 months of follow-up. Nearly two years after introduction of the ban, a statistically significant level drop was observed; however, this abrupt drop in sales was also observed among all food categories, signifying a system-wide influence not captured in our available sales data. Further, during the 2012-2019 study time period, the SSB sales ban was part of a multicomponent healthy dining initiative, which included removal of fryers from cafeteria kitchens, reductions in trans-fat and salt content in food offerings, nutrient labeling on all items, and the introduction of healthful "grab and go" bowls that were deeply discounted. Thus, it is possible the sales decrease could have resulted from the cumulative outcome of these dining overhaul changes. However, the abrupt drop in sales, instead of a gradual tapering, more likely points to some uncaptured shock to the system outside of our available data.

For many organizations, some revenue loss can be a reasonable expectation, if not an inevitable consequence, of restricting sales of SSBs. Despite the system-wide drop in sales among all food venue categories, we can take two lessons from these findings. First, the typical follow up of most studies evaluating SSB bans may be too short to adequately assess the net impact of this type of policy: we observed increases in revenue through 18 months post-ban, demonstrating the economic sustainability of this practice. Second, despite the sharp decrease in all sales in late 2016, the long-term data demonstrate that the trend stabilized immediately, establishing a baseline mean monthly revenue of nearly $\$ 240,000$. Had the follow-up period been shorter (e.g., 24 months), the results may have seemed too volatile to conclude revenue stabilization; our 56-month post-ban follow up 146 
demonstrated eventual stabilization of the gross trend. Thus, a long-term follow up can be a valuable insight for food service managers who are concerned that such practices to be too unpredictable or too costly to sustain permanently.

Another important finding is the sustained decrease of SSB sales despite the availability of diet SSBs after the ban. While diet SSBs remained available for purchase as part of an incremental strategy, diet sodas did not experience an increase in purchasing as evidenced by the post-ban sales data, suggesting one of two things: either consumers who drank regular SSBs switched to healthier options (instead of opting to substitute their regular soda with the diet version), or the proportion of diet SSB drinkers decreased in the post-ban period. Either outcome resulted in consumers who replaced their SSB purchases with other beverage options. While the health system continued to offer diet soda to avoid criticism from employees and dining customers, our findings indicate that an all-soda ban may not have been as disruptive as initially expected. Further, an all-soda ban would enable more precise measurement and outcomes reporting for future employers who adopt a similar ban. At the very least, one takeaway for employers who decide to remove regular soda from their worksites is to direct their food service managers to clearly label diet versions in their point-of-sales systems so as to enable precise measures during evaluation.

After the SSB ban, sales of water, juice and sports/energy drinks increased, with purchases shifting heavily toward water options. This was consistent with a broader national trend during the study period, wherein sparkling water beverages such as $\mathrm{La}$ Croix and Polar were experiencing high consumer demand (Nelson \& Zarracina, 2016). Qualitative interviews with food service managers revealed that concessionaires 
conducted focus groups among employees prior to the ban that indicated a strong preference for water options. As a result, the health system offered a wide variety of still, sparkling, and flavored water options within all venues, and sales counts reflected a broad array of water products purchased. This represents a lesson for prospective employers to solicit feedback to determine which beverage substitutions will have favorable reception.

A secondary takeaway is to pay attention to larger cultural trends in the beverage world and capitalize on such trends by supplying popular beverage options to substitute SSBs. Despite the increase in water sales, the rise in juice and sports/energy drink sales represented an enduring consumer preference for sugary beverages. The degree to which increases in sports/energy drinks $(+2.03 \%)$ represent spillover from the SSB ban warrants further qualitative examination.

\section{Strengths and Limitations}

There are a number of strengths and limitations of our study. This study examines worksites of one large U.S. health system within a single state, limiting the generalizability and transferability of findings. However, restricting venue enrollment to one state ensured a shared broader policy and economic context, which could have otherwise presented a confounding issue. Next, our study did not include sales from onsite vending machines within the health system due to significant data discrepancies. Despite the unavailability of these data, the SSB ban also applied to vending machines throughout the health system, such that vending machines were not a source of purchasing spillover. 
Another limitation is the lack of a comparator group in our time series analysis, which would have enabled a more robust analysis. However, an ITS analysis is the best available method for this type of observational data and is a strength of our study. Additionally, the health system opted to retain diet versions of sodas that were banned, potentially biasing our estimates towards no difference between pre- and post-ban due to the inability to parse between regular soda and diet soda in the pre-ban period. Finally, the unexplained shock to food and beverage sales in late 2016 introduces a competing event, a threat to the ITS design. However, revenues among all categories stabilized shortly after this shock, mitigating its influence on the rest of the follow up period. Notwithstanding these limitations, ours is one of a few studies that reports medium- to long-term revenue outcomes from retail venues as well as consumer purchasing shifts after the implementation of an SSB ban.

\section{Conclusion}

Our findings report no immediate revenue ramifications to an SSB ban as well as a purchasing shift toward more healthful beverage options throughout the remainder of the follow-up period. Despite no statistically significant revenue impact within 18 months of implementation, there was a sharp decrease in gross food and beverage sales approximately two years after the SSB ban. Our findings demonstrate that consumer purchasing habits shifted largely toward water and juice offerings after regular soda was restricted, despite the continued availability of diet soda options. Additionally, our study provides revenue impacts stratified by food venue size, type, and location, demonstrating the differential revenue impact that an SSB ban may have on food venues that differ by 
these characteristics. Future studies would benefit from obtaining long-term revenue trends as well as characteristic features of venues implementing similar SSB restrictions. 


\section{CHAPTER 6: THE ROLE OF ORGANIZATIONAL CONTEXT IN SHAPING HFEP POLICY ADOPTION AND IMPLEMENTATION CHALLENGES}

\section{Introduction}

From the perspective of new institutionalism, health care institutions promote health and well-being through the organization and delivery of health services (DiMaggio \& Powell, 1983). These organizations shape perceptions about healthy behaviors and health care leaders act as authoritative figures guiding policy and practice regarding all aspects of health. As public health crises mount and subside, society (e.g., governments, schools, employers) looks to these institutions to develop and disseminate new knowledge and evidence-based practices. As an example, when the health consequences of smoking became evident, hospitals modeled steps to prohibit indoor smoking. First, hospitals restricted the sale of cigarettes from onsite shops (Lesser, Cohen \& Brook, 2012). Next, hospitals began requiring smoking cessation as part of preoperative workup. This was followed by designating outdoor smoking areas and, ultimately, banning smoking outright on hospital campuses (Freedhoff \& Stevenson, 2008). These practices rippled into communities and worksites in a similar stepwise fashion, subsequently contributing to a downward trend of smoking prevalence among Americans (Drope et al., 2018). This example demonstrates the institutional leverage that hospitals wield in shaping public perception and modeling healthy behaviors for broader audiences and settings. Similarly, as we are confronted with another public health crisis, specifically diet-related chronic 
diseases, there is mounting pressure for health care institutions to signal the need for widescale change by modeling healthy food environments.

However, the extent to which these institutions have successfully transformed their internal food environments warrants further scrutiny. Poor food environments are ubiquitous (Hall, 2018), and health care cafeterias and cafés have been no exception (Champ et al, 2019; Malhotra, 2013; Lawrence et al, 2009; Wojcicki, 2013; Cram et al., 2002). Though the grey and refereed literature are replete with toolkits and techniques to implement healthy food environment policies (HFEPs), few practices have been evaluated in-situ. To the contrary, most implementation advice is crafted to be contextfree and applicable to as many settings as possible (CDC 2019; PHLC, 2013). In the event a HFEP is not successful within an organization, explanations most often conclude the practice was not implemented "correctly," as opposed to looking at the situational environment and speculating on the fit (Greenhalgh et al, 2017; Plsek, 2003).

Though implementation of HFEPs within health care organizations is growing, little is known about how organizational factors come together to shape the organizational context of an institution, and how these contexts shape the implementation process (Glisson \& Schoenwald, 2005; Greenhalgh et al, 2004). Context is determined by prospectively identifying determinants, or factors, that could influence the effectiveness of an intervention (Baker et al, 2015). Waltz and colleagues (2019) identified implementation challenges to advancing evidence-based interventions in real-world health care settings, arguing that strategies must be tailored to the local context of the organization. 
The absence or presence of certain organizational factors can result in distinct opportunities or challenges to changing the food environment: For example, a large organization may have adequate slack resources to implement HFEPs (advantage), yet it may struggle to reach and engage an executive champion who will lend ongoing buy-in (disadvantage) (Damanpour, 1992); similarly, a highly-centralized governance structure may streamline logistical support for HFEP execution (advantage), yet pose as a barrier when individual sites wish to exercise autonomy to capitalize on local HFEP opportunities (disadvantage) (Foss, Husted, Michailova \& Pedersen, 2003; Gulati, 1999). The perceived and actual costs of implementing HFEPs may differ based on an organization's information channels or organizational culture. Thus, it is not any given implementation factor that should be considered a barrier or facilitator of implementation; rather, a confluence of factors shapes the landscape of opportunities or challenges. The degree to which all of these factors play a real role in adoption and implementation vary in influence (Birken et al., 2015). Notwithstanding the growing acknowledgment that addressing contextual barriers is necessary (Greenhalgh et al, 2004), Bosch and colleagues (2007) observe that the translation of identified barriers into tailor-made solutions is still a "black box" from a practical perspective.

In this study, we sought to describe HFEP implementation and the organizational contexts of six health care organizations: 1) a state health agency; 2) a regional not-forprofit health care system; 3) an urban academic medical center; 4) a federally-run medical center; 5) a faith-based hospital network; and 6) a rural standalone hospital. By studying six diverse systems that adopted various HFEPs, our findings elucidate the role 
of the organizational context in selecting HFEPs and corresponding implementation challenges.

\section{Methods}

\section{Design \& Setting}

Six health care organizations in Oregon were recruited for study participation using a purposive sampling strategy; the rationale and criteria for enrollment have been described elsewhere (see Chapter 3). Ethical approval for this study was obtained from Portland State University Institutional Review Board (Study \#196741-18).

\section{Data Collection}

We used semi-structured key informant interviews to explore the selection and implementation processes of HFEPs and to understand the organizational context of participating organizations. Eleven interviews ranged 41-57 minutes in length and were conducted between December 2019 and March 2020. Before starting each interview, written consent was obtained from study participants, with respondents receiving a copy of the consent form as well as the interview protocol, which was developed using Kallio and colleagues' (2016) five-step protocol development framework (Figure 3.1). Interviews were transcribed verbatim, names were redacted, and all data files (interview transcripts, field notes and any supplemental documentation offered) were managed in NVivo 12 (QSR International, Cambridge, MA), a qualitative analysis software. 
In the interview protocol, respondents were asked to specifically outline HFEPs that their institution adopted, including pilots or trial runs to demonstrate HFEP feasibility, staff procedures, changes to the physical environment, vending changes and any educational materials developed for staff and consumers (see Questions 4A-F, 7A-C, and $8 \mathrm{~B}$ in Appendix A).

To capture HFEP selection and implementation, participants were asked to describe all HFEPs adopted at their institution, such as vending changes, reformulated recipes, healthier procurement policies or changes to the built layout (see Questions 4AF, 7A-C, and 8B in Appendix A). Specific HFEP prompts included practices identified in the Healthy Food Environment Policy Index as well as those practices that are widely described in the literature (operationalized in Table 6.1). For time efficiency, respondents were invited to provide supplemental documentation to be included as part of data collection materials.

\section{Table 6.1 Definition of Key Concepts}

\begin{tabular}{|l|l|l|}
\hline \multicolumn{1}{|c|}{ Concept } & \multicolumn{1}{|c|}{ Operational Definition } & \multicolumn{1}{c|}{ Source } \\
\hline $\begin{array}{l}\text { Health care } \\
\text { organization }\end{array}$ & $\begin{array}{l}\text { Any consumer-facing organization that is within the health care } \\
\text { industry, including hospitals, clinics, medical offices, and public } \\
\text { health agencies. Other health care organizations such as health } \\
\text { insurance companies, hospice homes and education centers are } \\
\text { excluded }\end{array}$ & $\begin{array}{l}\text { Institute of } \\
\text { Medicine, 2003 }\end{array}$ \\
\hline $\begin{array}{l}\text { Healthy Food } \\
\text { Povironment }\end{array}$ & $\begin{array}{l}\text { Any of the following policies instituted within the retail } \\
\text { environment: (1) trans-fat and/or sodium limits; (2) limiting or } \\
\text { banning access to sugar-sweetened beverages; (3) instituting } \\
\text { serving size limits; (4) prioritizing healthy methods of food } \\
\text { preparation; (5) nutrition labeling for meals and items at point of } \\
\text { service; (6) promotion of healthy foods and beverages in } \\
\text { marketing materials; (7) using pricing strategies to increase the } \\
\text { affordability of healthy items; (8) healthy vending policies; and } \\
\text { (9) other HFEP policies }\end{array}$ & $\begin{array}{l}\text { Environment } \\
\text { Policy Index, } \\
\text { INFORMAS, } \\
2014\end{array}$ \\
\hline $\begin{array}{l}\text { Organizational } \\
\text { context }\end{array}$ & $\begin{array}{l}\text { Four organizational domains: structure (governance, size, } \\
\text { interorganizational coupling); receptivity (tension for change, } \\
\text { norms and culture); resource availability (slack resources, social }\end{array}$ & $\begin{array}{l}\text { Greenhalgh, } \\
\text { 2004; Miller, } \\
\text { Droge \& } \\
\text { Toulouse, 1988 }\end{array}$ \\
\hline
\end{tabular}




\begin{tabular}{|l|l|l|}
\hline & $\begin{array}{l}\text { network location, information sharing); and leadership (change } \\
\text { agents, stakeholders, vision) }\end{array}$ & Rogers, 1995; \\
\hline $\begin{array}{l}\text { Adoption } \\
\text { decision }\end{array}$ & Steps and/or decisions made to implement healthy HFEPs & 2003 \\
\hline $\begin{array}{l}\text { Implementation } \\
\text { Process }\end{array}$ & $\begin{array}{l}\text { Any deliberately initiated attempt to introduce new, or to modify } \\
\text { existing, patterns of action in health care or some other formal } \\
\text { organizational setting. Deliberate initiation means that an } \\
\text { intervention is: institutionally sanctioned; formally defined; } \\
\text { consciously planned; and intended to lead to a changed outcome }\end{array}$ & May et al 2007 \\
\hline
\end{tabular}

Analysis

Employing a directed content analysis approach (Hsieh \& Shannon, 2005), an a priori codebook was developed based on a comprehensive literature review. Three domains of questions included organizational characteristics, selection and adoption of HFEPs, and the implementation process for each HFEP (Table 6.1). Multiple iterative cycles of deductive coding took place utilizing parent-child codes, with broad categories (parents) followed by smaller, more specific sets of codes (children).

To determine the organizational context of each institution during the analysis stage, we queried respondents as to the organizational governance structure, communication pathways, stakeholders, executive champions, and workplace culture (see Questions 9-11, 12A-C, 13-14A-B, and 15A-C in Appendix A). These questions regarding contextual characteristics were extensively reviewed in the organizational literature as part of the broader nature of this work (dissertation study). The following four domains and 11 variables represent the synthesis from the relevant organizational literature. These concepts provide the basis of the organizational context probes and Table 6.2 operationalizes these concepts. 


\section{Table 6.2 Operationalization of Organizational Context}

\begin{tabular}{|c|c|c|c|}
\hline Domain & Variable & Operational Definition & Source \\
\hline \multirow{3}{*}{ Structure } & Governance & $\begin{array}{l}\text { The use of institutional and structural authority } \\
\text { to collaborate, allocate resources, resolve } \\
\text { conflict, and control an organization through } \\
\text { decision-making and strategy formation. }\end{array}$ & $\begin{array}{l}\text { Williamson, 1991; } \\
\text { Jones et al., 1998; } \\
\text { Hill \& Lynn, 2004; } \\
\text { Provan \& Kenis, } \\
2008\end{array}$ \\
\hline & Size & $\begin{array}{l}\text { The number of employees within an } \\
\text { organization as well as operating budget. }\end{array}$ & $\begin{array}{l}\text { Aiken \& Hage, } \\
\text { 1971; Damanpour, } \\
1992\end{array}$ \\
\hline & $\begin{array}{l}\text { Inter- } \\
\text { organizational } \\
\text { coupling }\end{array}$ & $\begin{array}{l}\text { The degree to which an organization is } \\
\text { dependent on others within a network in order } \\
\text { to achieve goals; the degree to which } \\
\text { organizational processes, resources, and } \\
\text { decision-making are shared. }\end{array}$ & $\begin{array}{l}\text { Evan, 1965; Weick, } \\
1976\end{array}$ \\
\hline \multirow[t]{2}{*}{ Receptivity } & $\begin{array}{l}\text { Tension for } \\
\text { change }\end{array}$ & $\begin{array}{l}\text { The degree to which external pressures mount } \\
\text { within an organization; internal processes are } \\
\text { discordant with needs of the organization; and } \\
\text { imperative for organizational action is } \\
\text { heightened. }\end{array}$ & $\begin{array}{l}\text { Zajac \& Shortell, } \\
\text { 1989; Beer \& } \\
\text { Nohria, } 2004\end{array}$ \\
\hline & $\begin{array}{l}\text { Norms and } \\
\text { culture }\end{array}$ & $\begin{array}{l}\text { The professional values of an organization that } \\
\text { guide behaviors and practices; influence of } \\
\text { organizational climate on strategic decision- } \\
\text { making. }\end{array}$ & $\begin{array}{l}\text { Ott, 1989; Tosti \& } \\
\text { Jackson, } 2000\end{array}$ \\
\hline \multirow{3}{*}{$\begin{array}{l}\text { Resource } \\
\text { Availability }\end{array}$} & $\begin{array}{l}\text { Slack } \\
\text { resources }\end{array}$ & $\begin{array}{l}\text { Maintenance of excess organizational } \\
\text { resources in order to experiment with novel } \\
\text { technologies, course-correct organizational } \\
\text { strategy, and adjust to internal and external } \\
\text { needs. Typified into liquid, potential and } \\
\text { recoverable slack. }\end{array}$ & $\begin{array}{l}\text { Cyert \& March, } \\
\text { 1963; Bourgeois, } \\
\text { 1981; Meyer, 1982; } \\
\text { Singh, 1986; } \\
\text { Hollingsworth, } 2008\end{array}$ \\
\hline & $\begin{array}{l}\text { Social } \\
\text { network } \\
\text { location }\end{array}$ & $\begin{array}{l}\text { The "placement" of an organization within an } \\
\text { interorganizational community as it relates to } \\
\text { the degree of distance to tangible resources } \\
\text { and power; a "node" within a network of } \\
\text { coalitions, information and technology. }\end{array}$ & $\begin{array}{l}\text { Katz \& Kahn, 1978; } \\
\text { Tushman \& } \\
\text { Anderson, 1986; } \\
\text { Nelson, Adger \& } \\
\text { Brown, } 2007\end{array}$ \\
\hline & $\begin{array}{l}\text { Information- } \\
\text { sharing }\end{array}$ & $\begin{array}{l}\text { The presence or absence of reliable, open } \\
\text { channels of communication within and } \\
\text { between organizations for maximized } \\
\text { effectiveness, collaboration and goal } \\
\text { alignment. }\end{array}$ & $\begin{array}{l}\text { March \& Simon, } \\
\text { 1993; Alter \& Hage, } \\
\text { 1993; Lasker et al., } \\
\text { 2001; Baker, 2002 }\end{array}$ \\
\hline \multirow[t]{2}{*}{ Leadership } & Change agents & $\begin{array}{l}\text { Executive-level or management "champion" } \\
\text { that initiates, prioritizes and facilitates } \\
\text { organizational innovation; agenda-setters that } \\
\text { determine objectives and anticipate strategic } \\
\text { opportunity. }\end{array}$ & $\begin{array}{l}\text { Wharton, Long \& } \\
\text { Schwartz, 2008; } \\
\text { Fairholm, 2009; } \\
\text { Cohen et al., 2016 }\end{array}$ \\
\hline & Stakeholders & $\begin{array}{l}\text { Customers, shareholders, suppliers, } \\
\text { employees, the public; groups or individuals } \\
\text { with distinct priorities and values that shape }\end{array}$ & $\begin{array}{l}\text { Brammer \& } \\
\text { Millington, 2003; } \\
\text { Smith et al., 2013; }\end{array}$ \\
\hline
\end{tabular}




\begin{tabular}{|l|l|l|l|}
\hline & $\begin{array}{l}\text { organizational trajectories as well as } \\
\text { possibilities. }\end{array}$ & $\begin{array}{l}\text { Fernandez \& } \\
\text { Rainey, 2017 }\end{array}$ \\
\cline { 2 - 4 } & Vision & $\begin{array}{l}\text { Long-term “organizational intent"; strategic } \\
\text { conversion of organizational resources to } \\
\text { realize values through action; guiding } \\
\text { principle that shapes organizational culture } \\
\text { and strategy. }\end{array}$ & $\begin{array}{l}\text { Tosti \& Jackson, } \\
\text { 2000; Foster \& } \\
\text { Akdere, 2007 }\end{array}$ \\
\hline
\end{tabular}

During the interpretive phase, each participating organization was scored along the four domains: structure, receptivity, resource allocation and leadership. Adopting Krein and colleagues' (2010) coding scheme, we assessed each domain with a positive $(+)$, negative (-), or mixed (+/-) score (Table 6.3). Scores were derived based on respondent answers to corresponding questions and cited with supporting data. A positive score for an organizational characteristic would be interpreted as a facilitator of HFEP implementation. While the ranges of $(-)$ to $(+)$ mirror a generally continuous unfavorableto-favorable spectrum for HFEP development, some ranges indicated categorical responses (e.g., norms and culture, size), thereby precluding the development of a cumulative score for all domains. Instead, the aim of these scores was to serve as heuristic devices for sensemaking during content analysis.

\section{Table 6.3 Scoring for Organizational Context}

\begin{tabular}{|c|c|c|}
\hline \multicolumn{3}{|l|}{ Structure } \\
\hline Governance & rigid, formal & adaptive, casual \\
\hline Size & small & $(-)-(+/-)-(+)$ \\
\hline Interorganizational coupling & loose, mild & $(-)-(+/-)-(+)$ \\
\hline \multicolumn{3}{|l|}{ Receptivity } \\
\hline Tension for change & low & $(-)-(+/-)-(+)$ high \\
\hline Norms and culture & heterogeneous & $(-)-(+/-)-(+)$ uniform values \\
\hline \multicolumn{3}{|l|}{ Resource Availability } \\
\hline Slack resources & unavailable & $(-)-(+/-)-(+)$ available reserve \\
\hline Social network location & diffuse & $(-)-(+/-)-(+)$ concentrated \\
\hline
\end{tabular}




\begin{tabular}{|c|c|}
\hline Information sharing & unreliable, costly $(-)-(+/-)-(+)$ reliable, open \\
\hline \multicolumn{2}{|l|}{ Leadership } \\
\hline Change agents & $(-)-(+/-)-(+)$ multiple champions \\
\hline Vision & $(-)-(+/-)-(+)$ compelling \\
\hline Stakeholders & conflicting $(-)-(+/-)-(+)$ aligned \\
\hline
\end{tabular}

Supporting data for each assessment include quote attributions followed by organization identifier (Table 6.1) and an " $E$ " if the interview respondent was an executive leader or "O" for operational manager (e.g., 2E or 5O). These study methods are also reported using the Consolidated Criteria for Reporting Qualitative Research (COREQ), a formal reporting guideline consisting of 32 items describing the selection, methods, analysis and interpretation of study findings (Appendix D).

\section{Results}

\section{Descriptive characteristics}

General organizational characteristics are displayed in Table 6.4. This information was drawn from the interview transcripts and review of each organization's public website.

Table 6.4 Organizational Characteristics of Study Participants

\begin{tabular}{|c|c|c|c|c|c|c|}
\hline$a$ & $u_{1}$ & $A$ & $\omega$ & $N$ & - & ฐ) \\
\hline
\end{tabular}




\begin{tabular}{|c|c|c|c|c|c|c|}
\hline 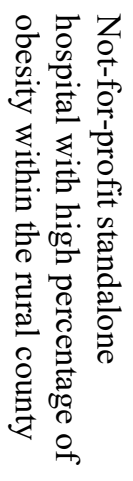 & 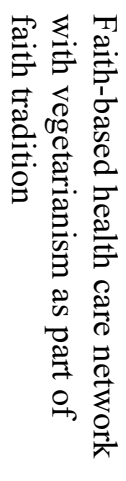 & 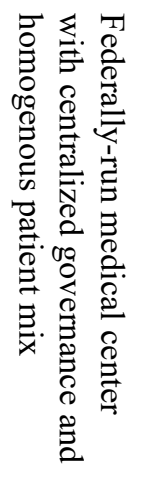 & 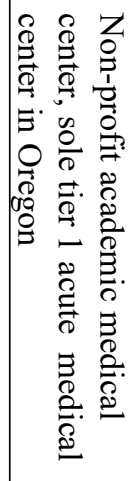 & 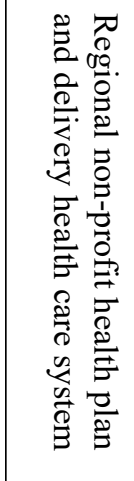 & 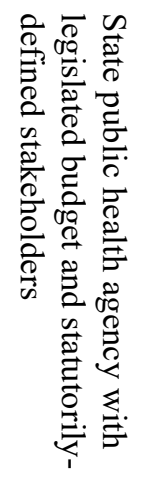 & 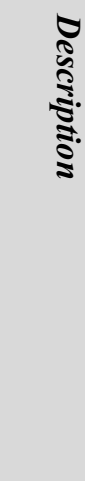 \\
\hline 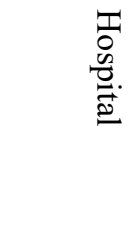 & 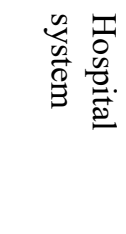 & 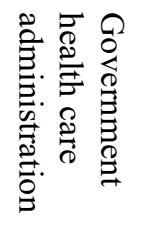 & 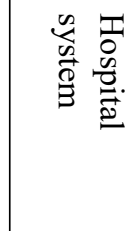 & 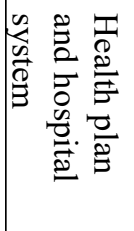 & 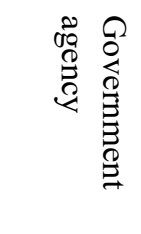 & 这 \\
\hline 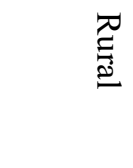 & 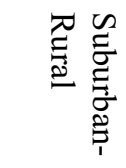 & 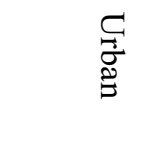 & $\begin{array}{l}\overparen{\Xi} \\
\stackrel{\Xi}{\Xi}\end{array}$ & 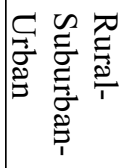 & 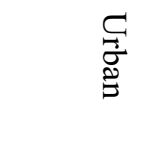 & 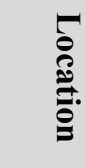 \\
\hline $\begin{array}{l}\tilde{U} \\
\stackrel{\mathscr{E}}{=}\end{array}$ & $\begin{array}{l}\mathscr{E} \\
\stackrel{\mathscr{E}}{=}\end{array}$ & $\begin{array}{l}\frac{3}{8} \\
\stackrel{3}{\Xi} \\
\text { : }\end{array}$ & 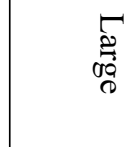 & $\underset{\substack{0 \\
0 \\
00 \\
0 \\
0}}{T}$ & 交 & \% \\
\hline- & $N$ & - & 6 & $\bar{\sigma}$ & $\triangle$ & 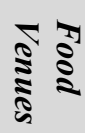 \\
\hline 岁 & $\begin{array}{l}N \\
\stackrel{8}{8}\end{array}$ & $\stackrel{\overrightarrow{8}}{\circ}$ & $\begin{array}{l}\overline{1} \\
\stackrel{0}{8} \\
8\end{array}$ & $\begin{array}{l}N \\
8 \\
8\end{array}$ & ' & है \\
\hline 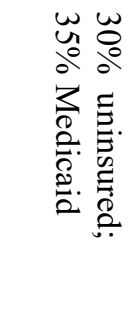 & 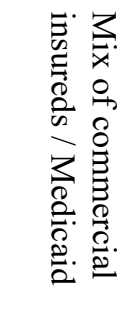 & 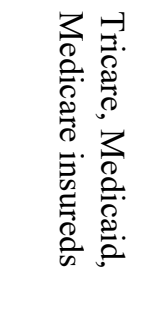 & 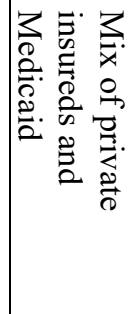 & 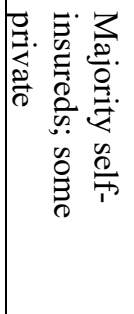 & 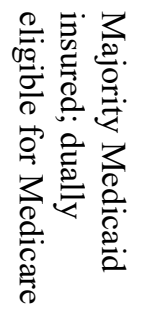 & $\begin{array}{l}3 \\
3 \\
3 \\
3 \\
3 \\
3\end{array}$ \\
\hline
\end{tabular}

Spectrum of HFEPs Adopted 
Across the six organizations, a total of 29 distinct HFEPs were identified (Table 6.5).

Practices ranged from committing to antibiotic-free poultry and salad bar vouchers to offering free cooking classes and removing sugar-sweetened beverages. Across all six, practices that most frequently overlapped included those that improve the quality/sourcing of foods (e.g., limits on sodium or trans fats), healthier methods of food preparation (e.g., replacing kitchen fryers with ovens), and adopting competitive pricing strategies (e.g., water priced less than soft drinks). All six organizations reported that their food service operations were subsidized by their host institution.

Table 6.5 Healthy Food Environment Policies Across Organizations

\begin{tabular}{|c|c|c|c|c|c|c|}
\hline \multirow[t]{2}{*}{ Healthy Food Environment Policy } & \multicolumn{6}{|c|}{ Organization } \\
\hline & 1 & 2 & 3 & 4 & 5 & 6 \\
\hline Antibiotic-free poultry & & & $\mathrm{x}$ & & & $\mathrm{x}$ \\
\hline Choice architecture & & & $\mathrm{x}$ & $\mathrm{x}$ & $\mathrm{x}$ & \\
\hline Codified nutritional standards & $\mathrm{x}$ & & $\mathrm{x}$ & & & $\mathrm{x}$ \\
\hline Competitive pricing strategies & & $\mathrm{x}$ & & $\mathrm{x}$ & $\mathrm{x}$ & \\
\hline $\begin{array}{r}\text { Consumer education/education } \\
\text { campaigns }\end{array}$ & & $\mathrm{x}$ & & & $\mathrm{x}$ & $\mathrm{x}$ \\
\hline Decoupling from fast food chain & & & & $\mathrm{x}$ & & \\
\hline Developing in-house Grab\&Go bowl & & $\mathrm{x}$ & & & & \\
\hline Employee wellness policy/committee & $\mathrm{x}$ & $\mathrm{x}$ & $\mathrm{x}$ & & & \\
\hline Farmer's market & $\mathrm{x}$ & & $\mathrm{x}$ & & & \\
\hline Free cooking classes & & $\mathrm{x}$ & & $\mathrm{x}$ & & $\mathrm{x}$ \\
\hline Healthy five-feet register policy & & & $\mathrm{x}$ & & $\mathrm{x}$ & \\
\hline Healthy meeting policy & $\mathrm{x}$ & & & & & \\
\hline Healthy vending policy/standards & & $\mathrm{x}$ & & $\mathrm{x}$ & & \\
\hline Hormone-free milk & & & $\mathrm{x}$ & & & $\mathrm{x}$ \\
\hline Improving food quality/sourcing & $\mathrm{x}$ & & $\mathrm{x}$ & $\mathrm{x}$ & $\mathrm{x}$ & $\mathrm{x}$ \\
\hline Labeling nutrient content/sticker & & $\mathrm{x}$ & $\mathrm{x}$ & & $\mathrm{x}$ & $\mathrm{x}$ \\
\hline Limits on comfort food specials & & & & & $\mathrm{x}$ & $\mathrm{x}$ \\
\hline Limits on sodium and trans-fats & $\mathrm{x}$ & $\mathrm{x}$ & & $\mathrm{x}$ & $\mathrm{x}$ & \\
\hline Monthly free salad bar voucher & & & $\mathrm{x}$ & & & \\
\hline Natural foods store & & & $\mathrm{x}$ & & & \\
\hline $\begin{array}{r}\text { Prioritizing healthy methods of food } \\
\text { preparation }\end{array}$ & & $\mathrm{x}$ & & $\mathrm{x}$ & $\mathrm{x}$ & $\mathrm{x}$ \\
\hline
\end{tabular}




\begin{tabular}{|c|c|c|c|c|c|c|}
\hline Routinely pilot diet trends & & & & $\mathrm{X}$ & & \\
\hline Robust monitoring and evaluation efforts & & & $\mathrm{X}$ & $X$ & $\mathrm{X}$ & \\
\hline Removing fryers from kitchen & & $\mathrm{X}$ & $\mathrm{x}$ & & $\mathrm{X}$ & $\mathrm{X}$ \\
\hline $\begin{array}{r}\text { Removing sugar-sweetened beverages } \\
\text { (SSBs) }\end{array}$ & & $\mathrm{x}$ & & & & \\
\hline Serving size limits & & & & & $\mathrm{X}$ & \\
\hline Subsidizing food service operations & $\mathrm{x}$ & $\mathrm{X}$ & $\mathrm{x}$ & $\mathrm{X}$ & $\mathrm{X}$ & $\mathrm{X}$ \\
\hline Sustainability commitment & & & & $\mathrm{x}$ & $\mathrm{x}$ & $\mathrm{x}$ \\
\hline Vegetarian kitchen/fare & & & & & $\mathrm{x}$ & \\
\hline
\end{tabular}

\section{Determining Organizational Contexts}

There was considerable variability in context across organizations, ranging from those that scored positively for all four domains to those that were generally negative across all domains. For example, Organization 3 had low tension for change, unreliable information and conflicting stakeholders that lead to generally negative scores, while Organization 2 had multiple champions, a compelling vision and access to slack resources to support HFEP implementation. Remaining sites had a mix of positive and negative contextual features (Table 6.6). Full scores for each variable can be viewed in Appendix E.

Table 6.6 Organizational Contexts of Health Care Institutions

\begin{tabular}{|c|c|c|c|}
\hline Identifier & $\begin{array}{l}\text { Organizational } \\
\text { context }\end{array}$ & HFEPs* & Relationship \\
\hline 1 & $\begin{array}{l}+/- \\
+\quad \text { Structure } \\
+\quad \text { Receptivity } \\
-\quad \text { Resource } \\
\text { availability } \\
-\quad \text { Leadership }\end{array}$ & $\begin{array}{l}\text { Codified nutritional } \\
\text { standards } \\
\text { Healthy meeting policy } \\
\text { Farmer's market } \\
\text { Employee wellness } \\
\text { committee }\end{array}$ & $\begin{array}{l}\text { High receptivity with } \\
\text { few resources led to } \\
\text { smaller scale } \\
\text { initiatives that } \\
\text { included few } \\
\text { stakeholders }\end{array}$ \\
\hline 2 & $\begin{array}{ll}+ & \text { Structure } \\
+ & \text { Receptivity } \\
+ & \text { Resource } \\
\text { availability } \\
+\quad \text { Leadership }\end{array}$ & $\begin{array}{l}\text { Restricted sale of SSBs } \\
\text { Removed fryers from } \\
\text { kitchens } \\
\text { Competitive pricing } \\
\text { strategies } \\
\text { Free cooking classes }\end{array}$ & $\begin{array}{l}\text { Strong vision with } \\
\text { multiple executive } \\
\text { champions led to } \\
\text { comprehensive dining } \\
\text { overhaul }\end{array}$ \\
\hline
\end{tabular}




\begin{tabular}{|c|c|c|c|}
\hline 3 & $\begin{array}{ll}+/- & \text { Structure } \\
-\quad & \text { Receptivity } \\
- & \text { Resource } \\
\text { availability } \\
-\quad \text { Leadership } \\
\end{array}$ & $\begin{array}{l}\text { Choice architecture } \\
\text { Nutritional labeling } \\
\text { Monthly salad bar } \\
\text { voucher } \\
\text { Farmer's market }\end{array}$ & $\begin{array}{l}\text { Heavily subsidized } \\
\text { food service operations } \\
\text { with low tension for } \\
\text { change led to } \\
\text { incremental practices }\end{array}$ \\
\hline 4 & $\begin{array}{l}\text { +/- } \\
\text { +/- }\end{array}$ & $\begin{array}{l}\text { Competitive pricing } \\
\text { strategies } \\
\text { Healthy vending policy } \\
\text { Pilot diet trends } \\
\text { Sustainability } \\
\text { commitment }\end{array}$ & $\begin{array}{l}\text { Centralized } \\
\text { information sharing } \\
\text { and high purchasing } \\
\text { power led to routine } \\
\text { large-scale pilots }\end{array}$ \\
\hline 5 & $\begin{array}{l}+/- \\
+\quad \text { Structure } \\
+\quad \text { Receptivity } \\
+\quad \text { Resource } \\
\text { availability } \\
+\quad \text { Leadership }\end{array}$ & $\begin{array}{l}\text { Improving quality of } \\
\text { foods } \\
\text { Limits on comfort foods } \\
\text { Robust } \\
\text { monitoring/evaluation } \\
\text { Consumer education }\end{array}$ & $\begin{array}{l}\text { Strong vision of food } \\
\text { as medicine with low } \\
\text { interorganizational } \\
\text { coupling resulted in } \\
\text { incremental practices }\end{array}$ \\
\hline 6 & 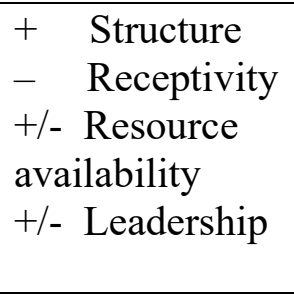 & $\begin{array}{l}\text { Sustainability } \\
\text { commitment } \\
\text { Hormone-free milk } \\
\text { Free cooking classes } \\
\text { Consumer education }\end{array}$ & $\begin{array}{l}\text { Strong long-term } \\
\text { champion with } \\
\text { concentrated social } \\
\text { network led to system } \\
\text { redesign of food } \\
\text { preparation processes }\end{array}$ \\
\hline
\end{tabular}

*Selected HFEPs showcase breadth of adopted practices; see Table 5 for exhaustive list.

Table 6.7 Supporting Documentation for Contextual Determinations

\begin{tabular}{|c|c|c|c|c|}
\hline \multirow[b]{2}{*}{ Identifier } & \multicolumn{4}{|c|}{ Domain } \\
\hline & $\begin{array}{l}\quad \underline{\text { Structure }} \\
\text { governance, size, } \\
\text { interorganizational } \\
\text { coupling }\end{array}$ & $\begin{array}{l}\text { Receptivity } \\
\text { norms and } \\
\text { culture, tension } \\
\text { for change }\end{array}$ & $\begin{array}{l}\quad \frac{\text { Resource }}{\text { Allocation }} \\
\text { slack resources, } \\
\text { social network } \\
\text { location, information } \\
\text { sharing }\end{array}$ & $\begin{array}{l}\text { Leadership } \\
\text { champion, } \\
\text { stakeholders, vision }\end{array}$ \\
\hline 1 & $\begin{array}{l}\text { "The Executive Order } \\
\text { and the creation of } \\
\text { [redacted]'s position } \\
\text { really was the foot in } \\
\text { the door to start } \\
\text { talking about this with } \\
\text { [redacted] again. In a } \\
\text { way to say, 'Hey, we }\end{array}$ & $\begin{array}{l}\text { "We didn't have } \\
\text { enough money to } \\
\text { get a ton of } \\
\text { resources out into } \\
\text { communities, so } \\
\text { we were } \\
\text { struggling getting } \\
\text { community policy }\end{array}$ & $\begin{array}{l}\text { "[In] Oregon, we } \\
\text { don't really have } \\
\text { funding to support } \\
\text { nutrition and } \\
\text { physical activity. } \\
\text { Period. Whether it's } \\
\text { at the local level or } \\
\text { the state level and }\end{array}$ & $\begin{array}{l}\text { "I think access to } \\
\text { decision-makers is } \\
\text { always challenging. } \\
\text { There's somebody I } \\
\text { could talk to from } \\
\text { [redacted] any day } \\
\text { of the week. But that } \\
\text { one person doesn't }\end{array}$ \\
\hline
\end{tabular}




\begin{tabular}{|c|c|c|c|c|}
\hline & $\begin{array}{l}\text { have these wellness } \\
\text { plans now, and } \\
\text { improving the food } \\
\text { environment is } \\
\text { explicit.", [1E] }\end{array}$ & $\begin{array}{l}\text { traction, so the } \\
\text { focus really } \\
\text { became us. And, if } \\
\text { we can get our } \\
\text { own house in } \\
\text { order, what would } \\
\text { that look like?" } \\
{[1 \mathrm{E}]}\end{array}$ & $\begin{array}{l}\text { not only is this not a } \\
\text { high priority, but this } \\
\text { nature of work is just } \\
\text { not funded. There are } \\
\text { no resources or staff } \\
\text { capacity to make it a } \\
\text { priority." }[10]\end{array}$ & $\begin{array}{l}\text { really have decision } \\
\text { making authority." } \\
{[1 \mathrm{O}]}\end{array}$ \\
\hline 2 & $\begin{array}{l}\text { “HR embraced it, } \\
\text { because we're a self- } \\
\text { insured employer, } \\
\text { right? They're always } \\
\text { promoting wellness, } \\
\text { because we want a } \\
\text { healthy workforce. The } \\
\text { structure facilitated } \\
\text { integration." [2E] }\end{array}$ & $\begin{array}{l}\text { "I would say } \\
\text { there was enough } \\
\text { of a ground swell, } \\
\text { not just from } \\
\text { nutrition folks, } \\
\text { but the physicians } \\
\text { and some of our } \\
\text { program areas." } \\
{[2 \mathrm{E}]}\end{array}$ & $\begin{array}{l}\text { "When we did the } \\
\text { budgeting, we } \\
\text { decided to bite the } \\
\text { bullet. But also, one } \\
\text { of things we did was } \\
\text { not charge rent to } \\
\text { our cafeterias. We } \\
\text { had to absorb that } \\
\text { impact. And we're a } \\
\text { big enough } \\
\text { organization, so } \\
\text { that's not } \\
\text { impossible." [2E] }\end{array}$ & $\begin{array}{l}\text { “...When we had } \\
\text { that leadership } \\
\text { support and } \\
\text { championing saying } \\
\text { this is what's going } \\
\text { to happen, they } \\
\text { were like 'Okay, } \\
\text { we'll figure it out } \\
\text { together.," [2O] }\end{array}$ \\
\hline 3 & $\begin{array}{l}\text { "In 2014-15, we } \\
\text { signed up with the } \\
\text { Partnership for a } \\
\text { Healthy America ... } \\
\text { We were one of the } \\
\text { initial signers, } \\
\text { actually, out of } 16 . \text { We } \\
\text { don't follow a lot of } \\
\text { that right now because } \\
\text { a lot of that is } \\
\text { unfortunately-you } \\
\text { have to pay money- } \\
\text { it's a weird program, it } \\
\text { kind of deviated from } \\
\text { what it started as." } \\
\text { [3E] }\end{array}$ & $\begin{array}{l}\text { "When the new } \\
\text { president came in, } \\
\text { he did all the } \\
\text { surveying of } \\
\text { people of what } \\
\text { mattered the } \\
\text { most. Food was } \\
\text { \#1. I went to my } \\
\text { boss and said, } \\
\text { "Are you } \\
\text { watching this? Do } \\
\text { you see that this is } \\
\text { a really great } \\
\text { opportunity for us } \\
\text { to like, basically, } \\
\text { be like here's } \\
\text { what we need?" } \\
\text { [3O] }\end{array}$ & $\begin{array}{l}\text { "I'll go to a meeting } \\
\text { and come back and } \\
\text { say, 'Here are all } \\
\text { these great facts and } \\
\text { figures, here's what I } \\
\text { think we should do.' } \\
\text { And they'll } \\
\text { [administration] be } \\
\text { like, 'Great, send us } \\
\text { some more } \\
\text { information.' And } \\
\text { nothing happens, that } \\
\text { dies. And I have no } \\
\text { budget, that's part of } \\
\text { the problem." [30] }\end{array}$ & $\begin{array}{l}\text { "For like a year } \\
\text { and a half, we went } \\
\text { through a year-and- } \\
\text { a-half visioning } \\
\text { process. Intense } \\
\text { visioning process. } \\
\text { And nothing ever } \\
\text { came of it. It was } \\
\text { like a giant exercise } \\
\text { that our boss's boss } \\
\text { made us do. Nobody } \\
\text { ever did anything } \\
\text { with it." [3O] }\end{array}$ \\
\hline 4 & $\begin{array}{l}\text { "[E]verything is } \\
\text { corporate. Years ago, } \\
\text { 10-15 years ago, each } \\
\text { site had much greater } \\
\text { local voice. So if you } \\
\text { were in Mississippi, } \\
\text { you're going to serve } \\
\text { certain fare, whatever. } \\
\text { What happened in the } \\
\text { last decade is that the } \\
\text { leadership at central } \\
\text { office wanted to make } \\
\text { [sites] consistent." } \\
\text { [4O] }\end{array}$ & $\begin{array}{l}\text { "The beauty of } \\
\text { working at } \\
\text { [redacted] is that } \\
\text { everyone here has } \\
\text { the goal of } \\
\text { serving our } \\
\text { [redacted]. The } \\
\text { trick is, 'OK, how } \\
\text { does this good } \\
\text { idea fit into the } \\
\text { list of other good } \\
\text { ideas and how do } \\
\text { you prioritize?' } \\
\text { Because there are }\end{array}$ & $\begin{array}{l}\text { "I have all kinds of } \\
\text { resources, from our } \\
\text { local dieticians who } \\
\text { serve in an evidence- } \\
\text { based way. I have } \\
\text { their national } \\
\text { counterpart, I have } \\
\text { the [redacted] and I } \\
\text { have their national } \\
\text { counterpart. I belong } \\
\text { to the American } \\
\text { College of } \\
\text { Healthcare } \\
\text { Executives and I can }\end{array}$ & $\begin{array}{l}\text { "I got an email } \\
\text { from our [redacted] } \\
\text { yesterday asking } \\
\text { how involved is our } \\
\text { [site] in the GEMS } \\
\text { program, which is } \\
\text { the Green } \\
\text { Environmental } \\
\text { Management } \\
\text { Service. Do we } \\
\text { compost our food? } \\
\text { Do we recycle? So } \\
\text { there is that } \\
\text { consciousness to be }\end{array}$ \\
\hline
\end{tabular}




\begin{tabular}{|c|c|c|c|c|}
\hline & & $\begin{array}{l}\text { so many good } \\
\text { ideas and so } \\
\text { much demand for } \\
\text { our attention and } \\
\text { energy that we } \\
\text { have to pick and } \\
\text { choose." [4E] }\end{array}$ & $\begin{array}{l}\text { reach out to them. } \\
\text { And so I think I have } \\
\text { a number of } \\
\text { stakeholder groups } \\
\text { that I could go to for } \\
\text { help." [4E] }\end{array}$ & $\begin{array}{l}\text { a good community } \\
\text { partner and also } \\
\text { take care of the } \\
\text { environment-you } \\
\text { know, higher goals } \\
\text { than just making } \\
\text { money."' }[4 \mathrm{O}]\end{array}$ \\
\hline 5 & $\begin{array}{l}\text { "I think because the } \\
\text { culture of } \\
\text { vegetarianism is part } \\
\text { of the [our] culture, } \\
\text { they understand that } \\
\text { you need to have } \\
\text { vegetarian proteins. } \\
\text { There is a Director's } \\
\text { team and we will share } \\
\text { recipes back and forth, } \\
\text { among all of the } \\
\text { [redacted] sites, where } \\
\text { Directors will share } \\
\text { whatever we're } \\
\text { looking for." [5O] }\end{array}$ & $\begin{array}{l}\text { "We want food to } \\
\text { be nourishing and } \\
\text { wholesome. So } \\
\text { the idea is that if } \\
\text { you want to come } \\
\text { to our cafeterias } \\
\text { to find something } \\
\text { nourishing and } \\
\text { healthful, it's } \\
\text { available to you. } \\
\text { but if you had a } \\
\text { crappy day and } \\
\text { want something } \\
\text { comforting, we } \\
\text { can offer that } \\
\text { too." [5O] }\end{array}$ & $\begin{array}{l}\text { "So my cafeteria is } \\
\text { one of the few } \\
\text { cafeterias of its size } \\
\text { that actually makes } \\
\text { money. It's very rare, } \\
\text { it's a profit center for } \\
\text { the hospital, we } \\
\text { made some money } \\
\text { last year. It's very } \\
\text { rare. Cafeteria sales } \\
\text { 2018, we made } \\
\$[\text { redacted]. That's } \\
\text { unheard of. We } \\
\text { expected it to cost } \\
\text { [redacted] a day to } \\
\text { stay open, but we } \\
\text { made [redacted]." } \\
\text { [50] }\end{array}$ & $\begin{array}{l}\text { "The CFO of our } \\
\text { hospital and our } \\
\text { region is-he never } \\
\text { loses sight of our } \\
\text { "Why" in what we } \\
\text { do what we do. So } \\
\text { he would never say, } \\
\text { 'We're going to } \\
\text { make all this crap } \\
\text { available because it } \\
\text { makes us a lot of } \\
\text { money.' Most of } \\
\text { our stakeholders, } \\
\text { we have } \\
\text { conversations } \\
\text { around } \\
\text { healthfulness of } \\
\text { food" [5E] }\end{array}$ \\
\hline 6 & $\begin{array}{l}\text { "I think our rural } \\
\text { location makes a lot of } \\
\text { things difficult-no } \\
\text { vendor comes this far } \\
\text { east... And I can't } \\
\text { make large orders, so } \\
\text { I'm left to find what I } \\
\text { need in town." [6O] }\end{array}$ & $\begin{array}{l}\text { "And the obesity } \\
\text { rate here is, we } \\
\text { are part of } 3 \text { other } \\
\text { counties that have } \\
\text { the highest } \\
\text { obesity rate in the } \\
\text { state. So, the } \\
\text { obesity rate } \\
\text { connected with a } \\
\text { lack of health } \\
\text { behaviors, that's } \\
\text { a challenge, you } \\
\text { know? It didn't go } \\
\text { over well when I } \\
\text { took the deep } \\
\text { fryer out of the } \\
\text { kitchen." [60] }\end{array}$ & $\begin{array}{l}\text { "We're not contract } \\
\text { food service or } \\
\text { anything, so I did } \\
\text { what I did and stayed } \\
\text { within my budget and } \\
\text { I was the decision- } \\
\text { maker. You know, I } \\
\text { got a lot of help from } \\
\text { the folks at HWH. } \\
\text { And at that time, I } \\
\text { knew some people } \\
\text { from Food Alliance." } \\
{[6 \mathrm{O}]}\end{array}$ & $\begin{array}{l}\text { "When I make a } \\
\text { change and } \\
\text { administration } \\
\text { goes, 'Don't do } \\
\text { that, change back,' } \\
\text { I tell them, 'Give it } \\
\text { three months.' } \\
\text { Because complaints } \\
\text { go away. Whenever } \\
\text { you make a change, } \\
\text { even if it's a good } \\
\text { change, you're } \\
\text { going to get a } \\
\text { certain number of } \\
\text { complaints. So you } \\
\text { don't react right } \\
\text { away because that's } \\
\text { a mistake." [6O] }\end{array}$ \\
\hline
\end{tabular}

\title{
Relationship between HFEPs and Organizational Contexts
}

\section{Organization 1 was a state health agency and thus had a legislated governance structure}

\author{
and statutorily defined stakeholders. HFEPs adopted included codified nutritional
}


standards for onsite vendors, a healthy meeting policy, a farmer's market, and a strong employee wellness committee that drove other initiatives targeting the food environment. Though the tension for change among employees of this mid-sized organization was high, resource constraints (e.g., lack of funding) and an absence of strong and consistent leadership were two contextual characteristics that shaped the enduring implementation challenge of lack of buy-in among other key stakeholders, such as licensed state vendors and the Commission for the Blind, which has a contractual right of first refusal for all public vending opportunities statewide.

Cross-organizational collaboration was often unsuccessful: "Even if someone within [redacted] was like, 'Yeah this is great, I'd love to work with you on it,' you know, three weeks go by between emails and missed phone calls because people just don't have enough time. And without the urgency, it continually falls off." [1O] Thus, HFEP development was primarily developed by interested state employees at their discretion, typically within the forum of the employee wellness committee. Funding for initiatives was typically obtained through grant applications, after which the employee wellness committee would convene to draft HFEP proposals.

Organization 2 was a regional non-profit health plan and delivery health care system that adopted a comprehensive range of HFEPs which included restricting sales of sugar-sweetened beverages, removing fryers from every food venue, competitively pricing healthful options, and subsidizing food service operations. Garnering strong positive scores for each of the four organizational domains, a dual commitment to worksite wellness as well as sustainability were the drivers of large structural changes to the food environment: 
"[Redacted] is different because we have a health plan and a delivery system altogether. When you have the health plan at the table and look at the numbers. And we're an integrated system. You can do things when you have all parts at the table, and realize that investment." [2E]

As the health system in this instance was also the health plan insurer, this aligned HFEP development along with incentives for costs savings through decreased employee medical spending. This resulted in exchanging incremental HFEPs for wide-reaching structural changes, such as removing sugar-sweetened beverages and fryers from cafeterias. Executive sponsorship of wide-reaching HFEPs was coupled with ongoing collaboration with the health system's dietetic department, which provided skill-sharing and logistical support. Finally, a formal sustainability commitment bolstered system-wide changes and employee pushback.

Organization 3 was an urban academic medical center, adopting HFEPs such as antibiotic-free poultry, choice architecture, nutritional labeling and a healthy five-feet register policy, which precluded competitive foods (also known as "junk foods") from being within five feet of checkout registers. This organization scored the lowest among participants in each of the four domains, with power struggles, mutual mistrust between staff and executive administrators, and resource constraints as persistent implementation challenges:

"During this entire time, we've had six different consulting companies come through that I have not hired - they're part of [redacted] and about every 3 years we got somebody coming in - and they'll tell people the same thing I've been telling them except they'll accept what the consultant says and not so much from me. [Laughs] Which I thought about quitting and then telling them what to do! Just kidding. I still need a check.” [3E]

"I had that thought today, honestly, 'Did they just hire me so that they could say we have a sustainability manager and we're doing good things?' ... Because I feel like, what's the 
point of me being here? Nobody listens, nobody cares, and there's nobody telling them that they need to listen." [3O]

In addition to mistrust and apathy, respondents also stated that a low prioritization of facilities upgrades and kitchen maintenance conveyed a messaged that "Food Service is usually considered to be at the bottom ... and I find that somewhat disturbing" [3E]. Nevertheless, HFEP selection was prioritized based on incremental improvements that could be made to the existing food environment—-from nutritional labeling to slowly transitioning to antibiotic-free poultry, an informal strategy of building upon existing practices and relationships provided the mechanisms for implementation.

Organization 4 was a federally-funded medical center with a food services operation that was routinely a pilot site to test new HFEP trends. Among its adopted HFEPs were a healthy vending policy, competitive pricing for healthy foods, and incorporation of choice architecture within the food venue layout. The highly centralized governance structure enabled significant purchasing power to negotiate favorable terms with vendors. A centralized corporate office was also a conduit for skill-sharing among operational and executive leaders experiencing implementation challenges:

"I have my colleagues around the country. That's the beauty of being in an integrated health care system, I can reach out to 150 others to say, 'Hey we're thinking about this, is there anyone else doing this? What are your barriers?' I don't have to go reinvent the wheel. I can beg borrow and steal!" [4E]

A high level of collaboration among employees and staff was the primary method of HFEP development. For example, when employees responded to workplace surveys complaining about a lack of food options during night shifts, the operational manager developed a healthy vending policy, which included the purchase of refrigerated vending 
units that dispensed pre-packaged sandwiches on demand. The additional labor for upkeep did not pose a barrier: "We got sandwiches and that means there's a shelf life so that's probably a little bit more work for the Canteen staff to make sure they're checking expiration dates” [4E]. Likewise, diet trends (adding Beyond Burger to menu of grill options; replacing pizza stands with pre-packaged sushi) were implemented through the central office, resulting in a more streamlined implementation process for Organization 4.

Organization 5 was a faith-based health care network with vegetarianism as part of its faith tradition. HFEPs adopted included choice architecture, serving size limits, high quality food sourcing, and maintaining a vegetarian kitchen. With a strong moral commitment to food as medicine, engaging in HFEP development had uniform buy-in among employees, physician staff, and executive leaders. A historical commitment to vegetarianism influenced the kinds of HFEPs selected and maintained:

"We teach here that a more plant-based lifestyle is good for the environment, good for our bodies, and that you should have a plant slant. As a result, I'm not going to serve meat here because I can't teach that through all of our outlets whether it's through [departments of] Nutrition, Health and Wellness, Community Engagement, and serve something different." [5E]

The long-term food services manager was reported as a robust champion of food quality, incorporating limits on comfort food specials, removing impulse items from the checkout registers, and maintaining profitability at her café: "Because food service is an area that always costs a hospital money, they were always subsidized by the hospital. But my cafeteria is one of the few cafeterias of its size that actually makes money" [50]. 
Organization 6 was a rural standalone hospital with a high uninsured/Medicaid patient population. HFEPs adopted included patient education/cooking classes, healthy methods of food preparation, and a sustainability commitment that led to antibiotic-free meat and elimination of BPA-lined cans and plastics in food service units. The longrunning operational manager was the champion of many HFEP efforts, stating:

"People were talking about taking baby steps toward this-that just doesn't work for me. If I'm going to make a change, I'm going to make a big change. Because with people, you make a little change, when you're not paying attention, they change back. Doing whatever they were doing."

Intersecting a commitment to sustainability with a champion with direct decision-making authority translated into café meals that regularly include fermented vegetables to promote healthy gut flora, replacing conventional Jell-O® with scratch-made bone broth for postoperative patients. These HFEPs, rarely reported on or discussed in the refereed literature, were typically motivated by the food service manager's commitment to improving the healthfulness of the cafeteria in order to address the high obesity rate at the county-level, a statistic they reiterated throughout the interview.

\section{Discussion}

Among six health care organizations, the spectrum of HFEPs varied considerably, with each organization's context influencing the implementation process. Even as some organizations overlapped in the kinds of HFEPs adopted, the implementation processes corresponded to the contextual opportunities and constraints of each respective institution. For example, let the reader consider the centralized governance models of Organizations 1 and 4: centralization was a facilitator of HFEP success in Organization 4 
due to institutional resource commitments. However, centralized governance was a barrier in Organization 1's HFEP development, as adverse stakeholders created roadblocks for needed approval pathways for HFEP opportunities. Likewise, a sustainability commitment was not effective in Organization 3 in promoting HFEP development but was a forceful driver of comprehensive food environment change for Organization 6 given its vocal executive champion. These HFEPs, examined in situ, illustrate how contexts, not implementation factors, determine the kinds of challenges that can be faced.

Organizations implemented HFEPs through champions, ongoing financial investment, and participation in national health care improvement collaboratives. For example, local champions played vital roles in implementation in all but Organizations 1 and 3. Three organizations acknowledged their participation in a national collaborative (e.g., Partnership for a Healthy America, Health Care Without Harm) as drivers of which HFEPs they selected to adopt. Organizations 1 and 2 used a top-down approach that minimally involved other stakeholders (e.g., vendors, consumers), when implementing their HFEPs. A formal sustainability commitment was a vehicle to drive HFEP adoption among some institutions, such as removing BPA-lined containers and plastics from food service operations [6O], serving less meat [2E], or promoting vegetarianism as "good for the environment" [5E].

To synthesize the relationships between each organization's context and HFEP selection, the following are contextual recommendations for organizations who align with one or more of the participating organizations but may be experiencing challenges with HFEP selection and implementation. 
Recommendation 1: When there are conflicting stakeholder views, focus HFEP development inward.

Improving the food environment often requires cooperation among various stakeholders; when consensus is not possible, HFEPs that require single stakeholder approval or HFEPs that can be approved within a single unit can be feasible steps toward improving the food environment. This recommendation synthesizes the experiences of Organizations 1 and 3. In the case of Organization 1, statutorily-appointed stakeholders created intractable barriers for some HFEP development opportunities, such as amending vendor procurement policies. Thus, adopted HFEPs were routinely the product of grantfunded endeavors (single stakeholder) as well as part of the worksite wellness initiative (single unit). The absence of a strong and visible champion led HFEP development to be perennially vying for prioritization for other state funds.

Similarly, mutual mistrust among parties in Organization 3 created unclear goals and vision, leading to HFEP adoption of smaller-scale initiatives that built upon existing practices. These HFEPs were incorporated into existing operations and did not require cooperation across agency stakeholders. Synthesizing these experiences, when largerscale HFEPs are not feasible due to divergent parties, adverse sentiment, or a lack of a unifying vision, adopting incremental HFEPs that can be developed within a smaller unit, requiring fewer stakeholders and buy-in, may be the more feasible strategy.

Recommendation 2: Mind the distribution of risk and reward when developing HFEPS. Often described as the "wrong pockets problem" in organizational theory literature, this problem occurs when one party bears the cost of developing and implementing a practice 
(in our case, HFEPs) that, if successful, will generate benefits for a different entity (Roman, 2015). In turn, the risk-bearing party will develop resentment toward the reward-accruing party, resulting in collaboration failure between parties. This recommendation synthesizes the experience of Organizations 2, 3 and 5. In Organization 2's case, it was both the employer as well as the health plan, and therefore could reap the benefits of their investments through implementation of comprehensive HFEPs. Conversely, in Organization 3, an operational manager was acutely aware of the opportunity costs associated with not having an earmarked fund:

"Because if I had a budget, I could prioritize different efforts every year. I can develop ideas, meals, pull expensive meats and replace them with delicious vegan meals-and I get those cost-savings back into my fund, allowing me to do more with that money."

For prospective organizational leaders contending with HFEP development, remaining mindful of which parties will be expected to incur costs and which parties will reap the benefit can be a useful heuristic to determine where and how to set aside funds for HFEP implementation and how to align incentives among stakeholders.

Recommendation 3: Learn from national HFEP collaboratives while engaging in local HFEP collaboration.

This takeaway synthesizes the experiences of Organizations 2, 4, and 6. Organization 4 turned to its $170+$ interorganizational partners frequently to reconcile implementation barriers, and Organization 2 selected HFEPs based on endorsements from similarly-sized partners in a national collaborative. Likewise, Organization 6 participated in skill-sharing among a group of food service managers who would provide updates of professional conferences and disseminate innovative ideas regarding HFEP development. Instead of 
committing significant upstart resources to research and strategy formation regarding HFEP development, each of these three organizations actively participated in ongoing efforts of larger collaboratives, whether internal or external.

The typical "costs" of search and information are also known as transaction costs (Williamson, 1989); these costs can differ among organizations given their resource availability, communication channels, and resource constraints. Coupled with environmental uncertainty and ambiguous external pressures (Lubell et al., 2017; Birken et al., 2015), learning from similarly-situated networks and sharing information was a recurring facilitator of HFEP selection and implementation among these organizations. Further, aspirations of engaging in local health system collaboration to bolster purchasing power with food distributors were reported by Organizations 1,3 , and 6 . If health systems could join collaboratively to streamline logistical processes in order for "institutional buyers in Portland to volume-buy" [3O] healthful foods with favorable terms, the historic need for health care organizations to subsidize food service operations could be ameliorated.

Overall, these recommendations are borne from the various experiences among these six health care organizations in Oregon. The contexts of these organizations were a platform for a diverse array of HFEPs to be implemented, and while a number of organizations overlapped in which HFEPs they implemented, each organization faced a distinct set of implementation challenges, which they addressed with varying mechanisms of implementation.

\section{Conclusion}


Our findings report the HFEPs and organizational contexts of six health care organizations. HFEP selection and development is closely tied to the contextual setting of the host organization, such that examining the structure, receptivity, resource availability and leadership characteristics can inform which HFEPs, and subsequent implementation strategies, can be used as a guiding framework to improve the health care food environment. As implementation of HFEPs within health care organizations is growing, selection is invariably linked with the context of the institution; as such, implementation design should be tailored closely to the context of the organization. 


\section{CHAPTER 7: CONCLUSION}

This concluding chapter offers a summary of the findings of this dissertation study. This summary includes a restatement of the study's purpose and a detailed description of the findings; assumptions and limitations; plans for dissemination of the findings; and implications for future research.

\section{Study Purpose and Findings}

This dissertation study sought to examine the relationship between organizational characteristics and the selection, implementation and outcomes of healthy food environment policies (HFEPs) within health care organizations. The overarching research question of this work was: How does the organizational context of health care organizations affect the implementation process and economic outcomes of healthy food environment policies? To accomplish this goal, this study utilized a mixed methods approach and had three aims:

1) Aim 1 sought to qualitatively describe the barriers to and facilitators of implementing HFEPs in five health care organizations: 1) a state health agency, 2) a regional non-profit health care system, 3) an urban academic medical center, 4) a federally-run medical center, and 5) a rural standalone hospital. Within this aim, there were three objectives:

a. To describe the implementation factors that facilitated HFEP implementation, 
b. To describe implementation factors that inhibited HFEP implementation, and

c. To compare and contrast the factors cited between operational and executive leaders of these participating organizations.

Aim 1 was accomplished through ten semi-structured interviews with health care leaders from the participating organizations. As HFEP implementation requires decision-making from executive-level as well as operational-level leaders, both of these positions were interviewed among each organization to understand their perspectives as to the implementation challenges and opportunities at their respective sites. In addition to examining the facilitators and barriers cited, differences in leaders' perceptions of the implementation challenges were also assessed to determine the degree of alignment, and if any discrepancy aggravated implementation challenges.

Across all organizations, 27 facilitators and 30 barriers were cited among respondents. These factors were collapsed into five and six broader categories, respectively: six facilitator categories were institutional commitment, employee wellness prioritization, technical assistance, incrementalism, external pressures to change the food environment, and presence of a champion. Five barrier categories were resource constraints, prescriptive centralization, complexity, pushback, and lack of leadership. Executives cited employee wellness prioritization as a facilitator to HFEP implementation twice as much as operational managers, whereas operational leaders predominantly cited technical assistance factors as facilitators, such as nutritional expertise and logistical support. 
Examining leadership perceptions, operational and executive leaders overlapped 44$75 \%$ when identifying facilitators but only $33-58 \%$ when identifying barriers to HFEP implementation. When leaders' perspectives diverged, executive over-delegation of decision-making and operational mistrust were two phenomena observed to aggravate implementation challenges. To overcome HFEP implementation barriers, three recommendations were synthesized by pairing facilitator and barrier categories: 1) Address 'lack of leadership' by identifying a 'champion' who will overcome organizational inertia; 2) Address 'complexity' barriers by finding 'technical assistance' factors; and 3) Overcome 'resource constraints' by solidifying 'institutional commitments' from the host organization.

2) Aim 2 sought to evaluate the revenue and consumer behavior outcomes of a sugar-sweetened beverage (SSB) ban established within one non-profit regional health care system. This quantitative inquiry had the following objectives:

a. To compare the monthly gross sales of all beverages before (FY 20122014) and after (FY 2015-2019) the introduction of the SSB ban;

b. To assess the percent change in sales by beverage type before and after the SSB ban; and

c. To conduct stratified analyses among the organization's 17 sites to determine if sites experienced a differential revenue impact based on size, type, or geographic location. 
Aim 2 was addressed by using an interrupted time series design to examine pre- and postban time periods for a difference in revenue. A piecewise linear regression model was used to assess the effect of the SSB ban on gross beverage sales. Among the organization's 17 food sites, the implementation of an SSB ban resulted in no immediate revenue impact among gross beverage sales at 6,12, and 18 months of follow-up but a statistically significant level drop nearly two years post-implementation. After the introduction of the SSB ban, the mean monthly revenue decreased from $\$ 273,570$ to $\$ 256,110$, representing a $6.38 \%$ decrease in beverage sales across the entire health system. Though diet sodas continued to be offered in lieu of regular soda, sites experienced a sharp decrease (-44.71\%) in all soda sales in the post-ban time period. This sharp decrease signifies that regular soda drinkers largely shifted to other beverages, and not to diet sodas, when the ban took effect.

After the removal of regular sodas, increases in water $(+35.14 \%)$, sports/energy drinks $(+2.03 \%)$, juice $(+7.45 \%)$ and coffee $(+1.84 \%)$ sales were observed, though only water and juice sale increases were statistically significant. Rural and urban sites experienced statistically significant mean monthly decreases after the ban, $-9.61 \%$ and $14.95 \%$, respectively. Suburban sites experienced slight increases in revenue in the postban period, though this increase was not statistically significant. When stratifying sites by size, both small and large sites observed significant losses in the post-ban period of $3.74 \%$ and $-9.62 \%$, respectively. 
3) Aim 3 sought to describe the relationship between organizational contexts and HFEP selection within six health care organizations: 1) a state health agency; 2) a regional not-for-profit health care system; 3) an urban academic medical center; 4) a federally-run medical center; 5) a faith-based hospital network; and 6) a rural standalone hospital.

This aim was accomplished with 11 interviews from two participants from each organization (only one respondent was available for Organization 6). During the interpretive phase, each participating organization was scored along the four domains to determine organizational context: structure, receptivity, resource allocation and leadership. Adopting Krein and colleagues' coding scheme (2010), we assessed each domain with a positive $(+)$, negative $(-)$, or mixed (+/-) score, citing participants' responses as supporting data. There was considerable variability in context across organizations, ranging from those that scored positively for all four domains to those that were generally negative across all domains.

The spectrum of HFEPs and organizational contexts varied considerably yet were closely tied. Even as some organizations overlapped in the kinds of HFEPs adopted, the implementation processes corresponded to the contextual opportunities and constraints of each respective institution. As an example, both Organizations 1 and 4 had centralized governance models, yet centralization was a facilitator of HFEP success in Organization 4 but a barrier in Organization 1. Likewise, a sustainability commitment was not effective in Organization 3 in promoting HFEP development but was a forceful driver of comprehensive food environment change for Organization 6. Thus, the selected HFEPs, 
examined in situ, illustrated how contexts, not implementation factors, determined the kinds of challenges each organization faced. Three recommendations were developed from the main findings:

1) When there are conflicting stakeholder views, focus HFEP development inward (synthesizing experiences of Organizations 1 and 3);

2) Mind the distribution of risk and reward when developing HFEPs (synthesizing experiences of Organizations 2,3, and 5); and

3) Learn from national HFEP collaboratives while engaging in local HFEP collaboration (Organizations 2, 4, and 6).

These recommendations were borne from a synthesis of participating organizational experiences. HFEP selection and development was closely tied to the contextual setting of the host organization, such that examining the structure, receptivity, resource availability and leadership characteristics to inform which HFEPs could be used as a guiding framework to improve the health care food environment.

\section{Synthesis of the Dissertation Papers}

Examined together, these three papers provide meaningful theoretical and practical insights into the selection, implementation, and outcomes of HFEP development. First, the theoretical synthesis: during the proposal stage of this dissertation study, Rogers' diffusion of innovations model conceptually framed the study aims and objectives. The investigator argued that an implementation science framework that emphasized the context of the setting would adequately capture the process of HFEP development, as the diverse organizational structures of health care organizations meant that the site of 
application needed to be explicitly addressed. Further, as the popularity and uptake of HFEPs was growing, the 'diffusion of innovations' element of the model was another attractive and relevant attribute of choosing this model. Examining which HFEPs would be best applied in which setting, described as innovation-system fit in the proposal, would add to the implementation science literature. The adapted model addressed how the environmental context (e.g., professional norms, public opinion) and organizational context (i.e., structure, resource availability, culture and leadership) would act as inputs when determining which HFEP to adopt.

At the completion of this work, the investigator now argues that this study is better understood through a different lens: the Donabedian model (1966). Borne from a journal article entitled, "Evaluating the Quality of Medical Care," the original framework was created as a quality assessment tool for health services research. Since its introduction, the model has been adapted in numerous studies and the original paper has been one of the most cited articles in the public health field. Briefly, the structureprocess-outcomes framework describes how system characteristics (structure) influence the sum of actions (process) that lead to effects on health care (outcomes). For this study, the structure-process-outcomes model can organize each study aim as follows: Aim 3 represents the structure element of the model, wherein the organizational characteristics that made up the context of the organization determined which HFEPs were subsequently selected by the organization; Aim 1 is the process element, as this aim described the facilitators of and barriers to the HFEP implementation process, while also underscoring the influence of leadership perspectives on this process; lastly, Aim 2 represents the outcome component of the model, wherein the introduction of a sugar-sweetened 
beverage ban, one prominent HFEP, was studied to determine the revenue implications as well as the consumer demand shifts in response to the ban.

Certainly, the Donabedian model is not without limitations. First, the prescriptively linear nature of this model is too simplistic to capture the iterative cycles of how a facilitator could influence structures and vice versa over time. Second, the Donabedian model does not account for external (e.g., environmental) pressures, as it contends with the infrastructure of the system alone in shaping the process. Notwithstanding these two limitations, a structure-process-outcomes model that is theoretically rooted in the health care field more saliently addresses these aims in relation to each other, and perhaps more so than an implementation science framework which omits the healthcare-specific emphasis of this work.

As to the practical insights of these papers, Chapters 1 and 2 initially discussed how the selection and implementation of HFEPs have historically underestimated the importance of organizational theory, with implementation toolkits and step-by-step guides often mentioning the need for a "champion" or "resources" but saying little else about the role of the organizational setting in determining which HFEPs would be better suited in what setting or how to overcome contextual barriers. The two qualitative aims of this dissertation study introduced a rich examination of both the contexts of six diverse health care organizations (Aim 3) as well as the facilitators and barriers cited during the HFEP implementation processes of these institutions (Aim 1). The combined findings of these aims will enable hospital administrators and food service managers to observe this process from beginning to end in six distinct organizations. A food service manager can then conduct an internal assessment of their own institutional context, determine which 183 
organization most closely matches its characteristics, and learn about which HFEPs were successful. The ability to showcase nuanced experiences from health care entities in order to help prospective health care leaders develop feasible strategies to improve their own food environments has been the ultimate goal of this dissertation work. As a clear understanding of one's organizational terrain is an essential first step in this process, the synthesis of this work tells us something that goes beyond individual papers: HFEP selection and implementation is a highly nuanced process that is dependent on both organizational characteristics as well as alignment among leaders overseeing this process. When complementing the qualitative inquiries with the quantitative findings of Aim 2 , the findings provide evidence to prospective health care leaders as to the revenue and consumer behavior outcomes of a sugar-sweetened beverage ban, a HFEP that is growing in popularity and uptake. By blending these process-oriented and outcome-oriented queries, prospective decision-makers can feel equipped and well-informed to proceed with HFEP selection, implementation, and evaluation.

\section{Assumptions and Limitations}

This dissertation work was accomplished using a set of assumptions. Prior to the onset of the study, the investigator assumed that relevant participants could be found to discuss their experience in the selection, implementation and outcomes of HFEPs within their health care organization. This was correct: among each of the six health care organizations prospectively identified, the investigator was able to get a rich, nuanced description of the types of HFEPs pursued as well as the opportunities and challenges experienced by each of the organizations. The second assumption made was that the 
investigator could successfully recruit two participants from each organization. This was incorrect, as only participant was available and willing to share Organization 6's HFEP process. As a result, modifications needed to be made in the number of organizations included in the analysis for Aim 3. The final assumption was that the investigator would be able to access and evaluate one organization's SSB ban and determine the revenue and consumer impacts after the introduction of the SSB ban. This assumption was met, as the investigator was able to execute a data-sharing memorandum of understanding with the relevant leaders within this organization.

Accordingly, there are also limitations to the findings of this study. The first limitation speaks to the generalizability of these findings: this entire dissertation work has been based on the experiences of six health care organizations recruited from one state, Oregon. This limits the transferability of findings to out-of-state organizations that face different policy contexts. The progressive political leaning and culture of Oregon shaped consumer demand, public perception, organizational norms, and health care leaders' opportunities; thus, novel implementation challenges not present in Oregon could have been missed.

The second limitation of this work applies to Aim 1 and 3: the single coder approach during qualitative thematic analysis. This limitation was unavoidable given the nature of this work, which is a dissertation study. However, this was largely mitigated by active dissertation committee oversight, with one mentor (RB) having specific expertise regarding the methodology, analytic techniques, and the qualitative software (NVivo) used. 
The third limitation was sampling bias, which was borne from the purposive sampling strategy utilized in this dissertation study. Relevant organizations for study inclusion were identified prospectively through a search of local media, grey literature, and websites of health care organizations in Oregon to determine whether policies were in place to enhance retail food environments. Thus, organization selection was done based on key informant recommendations and review of media coverage, which may have missed any organizations that implemented HFEPs but did not share publicly or widely of this decision.

Further, sampling bias was likely present in the selection of interview respondents: the investigator relied on executives from each institution to identify the most relevant operational counterpart to share their experience of HFEP implementation. This strategy was chosen because the executive leader would be best equipped to identify, and more importantly, connect the researcher to operational staff that may otherwise not respond, or decline an outside request for an interview. However, relying on the executive for a recommendation carried the risk of sampling bias, since the executive could have selectively chosen operational staff that were politically aligned with the executive. Thus, the investigator asked each executive for a list of "suggested" operational staff, reserving the right to extend an interview request to staff not included on the list.

A fourth limitation of this work was confirmation bias. The investigator was an academically trained doctoral student who conducted a thorough review of the relevant literature before conducting the study. The selection of the conceptual and theoretical frameworks, types of interview questions, and interpretative analysis have all been 
informed by the broader organizational and implementation science literature work that preceded this study. As such, novel phenomena may have been missed in exchange for a search of existing trends cited in the literature. The investigator attempted to mitigate this by incorporating the use of contemporaneous field notes during each interview to enable inductive analysis. While the literature and conceptual framework may have suggested possible factors related to HFEP implementation, field notes ensured that the analysis did not rely too heavily on the conceptual framework alone.

\section{Dissemination Plan}

The purpose of this work was to build upon existing knowledge and offer participating health care organizations, as well as prospective organizations, a more robust approach to improving food environments. The communication of research findings typically takes place in the form of refereed journal articles and conference presentations (Barnes et al., 2003). For this dissertation study, in addition to the investigator attempting to publish the findings of each of the three aims, the findings will also be incorporated into a customized report for each of the six participating organizations. The custom reports will be distributed before publication of the final aim. This positions the practice of disseminating findings as an integral component of the research process (Keen \& Todres, 2007).

There are several benefits of communicating findings to research subjects. First, participating organizations may be presented with new information that may have otherwise been unknown. Second, a dissemination plan can bolster subject receptivity to the merits of the research study, which was a useful incentive during recruitment. Third, a 
communication plan that summarizes the study findings may increase the probability that recommendations are put into practice, since it relieves the study participant from independently attempting to find the referred journal articles. Fourth, and most importantly, communicating results can instill participating organizations with a positive association with participation in research studies, priming future participation in future research endeavors.

\section{Implications for Future Research}

The obesity epidemic is a decades-long public health crisis with many causal determinants. As this work highlighted the importance of addressing poor food environments, the setting for this study was in the health care sector, anchored in the perspective that health care organizations have been pivotal for dissemination of many public health interventions throughout the past fifty years. While much of the work regarding HFEP implementation has been shaped to be context-free and broadly applicable to many settings, this study described HFEP selection and implementation and their relationship to their host institution, carefully examining the relationship between organizational contexts (Aim 3) and leadership perspectives (Aim 1) in shaping the implementation process. However, the health care sector possesses attributes that are particular to its field, namely the institutional responsibility to promote health and healing. The values of this sector are rooted in healthfulness, such that a different sector without any moral or societal obligation to promote health may have introduced different organizational elements. Thus, future work in different sectors (outside of health care) 
that examine organizational characteristics during HFEP implementation will move this field forward.

Additionally, the second aim of this work evaluated one institution's SSB ban in terms of revenue and consumer behavior impact. While this added to a very established literature examining revenue outcomes of HFEPs such as SSB bans, future work that prospectively designs the evaluation plan before any HFEP implementation will enable more precise and accurate measurement of the economic impact. As institutional decision-makers typically over-estimate the costs to implement public health interventions, accurate and reliable estimates will confer a confidence among champions to promote healthful interventions to improve their organizational food environments. 


\section{References}

Aase, S. (2011). Hospital foodservice and patient experience: what's new? Journal of the American Dietetic Association, 111(8), 1120.

Abramowitz, J., \& O'Hara, B. (2017). New estimates of offer and take-up of employersponsored insurance. Medical Care Research and Review, 74(5), 595-612.

Afshin, A., Micha, R., Khatibzadeh, S., Schmidt, L. A., \& Mozaffarian, D. (2014). Dietary policies to reduce non-communicable diseases. The Handbook of Global Health Policy, 1.

Aguirre, P. (2009). An anthropological view of the impact of poverty and globalization on the emerging epidemic of obesity. Diabetes in Women. Humana Press.

Aiken, M., \& Hage, J. (1971). The organic organization and innovation. Sociology, 5(1), 63-82.

Aldrich, H. E., \& Pfeffer, J. (1976). Environments of organizations. Annual review of sociology, 2(1), 79-105.

Allen, J. A., Duke, J. C., Davis, K. C., Kim, A. E., Nonnemaker, J. M., \& Farrelly, M. C. (2015). Using mass media campaigns to reduce youth tobacco use: a review. American Journal of Health Promotion, 30(2), e71-e82.

Almeida, F. A., Wall, S. S., You, W., Harden, S. M., Hill, J. L., Krippendorf, B. E., \& Estabrooks, P. A. (2014). The association between worksite physical environment and employee nutrition, and physical activity behavior and weight status. Journal of occupational and environmental medicine/American College of Occupational and Environmental Medicine, 56(7), 779.

Alter, C., \& Hage, J. (1993). Organizations working together (Vol. 191). Sage Publications, Inc.

American Academy of Pediatrics (AAP). (2010). Prevention and Treatment of Child Overweight and Obesity: Policy Opportunities Tool.

American Hospital Association (AHA). (2012). A Call to Action: Creating a Culture of Health. Advocacy Issues. American Hospital Association. Retrieved 28 May 2019 from: http://www.aha.org/advocacy-issues/healthforlife/culture.shtml.

American Medical Association. (2011). AMA Support for Breastfeeding. American Medical Association Policy Finder; H-245.982.

Ammendolia, C., Côté, P., Cancelliere, C., Cassidy, J. D., Hartvigsen, J., Boyle, E., ... \& Amick, B. (2016). Healthy and productive workers: using intervention mapping to design a workplace health promotion and wellness program to improve presenteeism. BMC Public Health, 16(1), 1190.

Anderson, L. M., Quinn, T. A., Glanz, K., Ramirez, G., Kahwati, L. C., Johnson, D. B., ... \& Katz, D. L. (2009). The effectiveness of worksite nutrition and physical activity interventions for controlling employee overweight and obesity: a systematic review. American journal of preventive medicine, 37(4), 340-357.

Andrade, A. M., Greene, G. W., \& Melanson, K. J. (2008). Eating slowly led to decreases in energy intake within meals in healthy women. Journal of the American Dietetic Association, 108(7), 1186-1191.

Andreyeva, T., Blumenthal, D. M., Schwartz, M. B., Long, M. W., \& Brownell, K. D. 
(2008). Availability and prices of foods across stores and neighborhoods: the case of New Haven, Connecticut. Health Affairs, 27(5), 1381-1388.

Andreyeva, T., Long, M. W., \& Brownell, K. D. (2010). The impact of food prices on consumption: a systematic review of research on the price elasticity of demand for food. American journal of public health, 100(2), 216-222.

Andreyeva, T., Luedicke, J., \& Wang, Y. C. (2014). State-level estimates of obesityattributable costs of absenteeism. Journal of occupational and environmental medicine/American College of Occupational and Environmental Medicine, 56(11), 1120.

Antos, J., Bertko, J., Chernew, M., Cutler, D., Goldman, D., McClellan, M., ... \& Shortell, S. (2009). Bending the curve: effective steps to address long-term health care spending growth. American Journal of Managed Care, 15(10), 676-680.

Arabin, B., Timmesfeld, N., Noever, K., Behnam, S., Ellermann, C., \& Jenny, M. A. (2019). How to improve health literacy to reduce short-and long-term consequences of maternal obesity?. The Journal of Maternal-Fetal \& Neonatal Medicine, 32(17), 2935-2942.

Arghode, V. (2012). Qualitative and Quantitative Research: Paradigmatic Differences. Global Education Journal, 2012(4).

Aronne, L. J. (2002). Obesity as a disease: etiology, treatment, and management considerations for the obese patient. Obesity, 10(S12), 95S.

Arizona Department of Education. (2005). Arizona Healthy School Environment Model Policy Implementation Pilot Study. Arizona Department of Education. Retrieved June 62019 from: http://www.azed.gov/ health-safety/cnp/teamnutrition/20032004/results2003-2004/ FmalReport-FullSummary.pdf.

Arrow, K. J. (1973). Information and economic behavior. (No. TR-14). Harvard University: Cambridge, MA.

Ashe, M., Graff, S., \& Spector, C. (2011). Changing places: policies to make a healthy choice the easy choice. Public Health, 125(12), 889-895.

Bailey, M. (2016). More hospitals are refusing to sell sugary drinks. And that's angering some workers. Stat News. Retrieved 2 Feb 2020 from: https://www.statnews.com/2016/10/24/hospitals-selling-sugary-drinks/

Bailey, R. L., Wang, T., \& Kaiser, C. K. (2018). Clash of the primary motivations: Motivated processing of emotionally experienced content in fear appeals about obesity prevention. Health communication, 33(2), 111-121.

Baker, K. A. (2002). Organizational communication. Journal of Business Communication, 11(1), 69-86.

Baker, R. L. (2017). Primary care and mental health integration in coordinated care organizations. Dissertations and Theses. Paper 3616. Retrieved 1 May 2019 from: https://pdxscholar.library.pdx.edu/open_access_etds/3616.

Baker, R., Camosso-Stefinovic, J., Gillies, C., Shaw, E. J., Cheater, F., Flottorp, S., ... \& Godycki-Cwirko, M. (2015). Tailored interventions to address determinants of practice. Cochrane Database of Systematic Reviews, (4).

Baker, K. A. (2002). Organizational communication. Journal of Business Communication, 11(1), 69-86. 
Bamford, C., Heaven, B., May, C., \& Moynihan, P. (2012). Implementing nutrition guidelines for older people in residential care homes: a qualitative study using Normalization Process Theory. Implementation Science, 7(1), 106.

Barberio, J. (2017). 10 Companies with Incredible Office Health and Wellness Zones. Working Mother. Retrieved Nov 12018 from: https://www.workingmother.com/10-companies-with-health-and-wellness-zones.

Barker, D. J. P. (1998). Mothers, babies, and health in later life. Elsevier Health Sciences.

Barnes, M. (2010). Solving the problem of childhood obesity within a generation. White House Task Force on Childhood Obesity Report to the President. Washington, DC.

Barnes, V., Clouder, D. L., Pritchard, J., Hughes, C., \& Purkis, J. (2003). Deconstructing dissemination: dissemination as qualitative research. Qualitative Research, 3(2), $147-164$.

Barry, C. L., Brescoll, V. L., \& Gollust, S. E. (2013). Framing childhood obesity: How individualizing the problem affects public support for prevention. Political Psychology, 34(3), 327-349.

Barry, C. L., Jarlenski, M., Grob, R., Schlesinger, M., \& Gollust, S. E. (2011). News media framing of childhood obesity in the United States from 2000 to 2009. Pediatrics, 128(1), 132-145.

Basit, T. (2003). Manual or electronic? The role of coding in qualitative data analysis. Educational research, 45(2), 143-154.

Basu, S., Jacobs, L. M., Epel, E., Schillinger, D., \& Schmidt, L. (2020). CostEffectiveness Of A Workplace Ban On Sugar-Sweetened Beverage Sales: A Microsimulation Model: A simulation model estimates the employee health and health care spending impacts of a workplace ban on sugar-sweetened beverage sales. Health Affairs, 39(7), 1140-1148.

Bauman, A., \& Chau, J. (2009). The role of media in promoting physical activity. Journal of Physical Activity and Health, 6(s2), S196-S210.

Baylor, Scott \& White Media Relations (Baylor). (2012). Employee Wellness News: Healthier Options Now at Baylor Cafes. Online Newsroom. Baylor Scott and White Health. Retrieved 21 July 2019 from: http://news.bswhealth.com/pages/thrive-baylor-cafe.

Beer, M., \& Nohria, N. (Eds.). (2000). Breaking the code of change (Vol. 78, No. 3, pp. 133-141). Boston, MA: Harvard Business School Press.

Bell, C., Pond, N., Davies, L., Francis, J. L., Campbell, E., \& Wiggers, J. (2013). Healthier choices in an Australian health service: a pre-post audit of an intervention to improve the nutritional value of foods and drinks in vending machines and food outlets. BMC health services research, 13(1), 492.

Bell, C. G., Walley, A. J., \& Froguel, P. (2005). The genetics of human obesity. Nature reviews genetics, 6(3), 221.

Berg, C., \& Forslund, H. B. (2015). The influence of portion size and timing of meals on weight balance and obesity. Current obesity reports, 4(1), 11-18.

Bernal, J. L., Cummins, S., \& Gasparrini, A. (2017). Interrupted time series regression 
for the evaluation of public health interventions: a tutorial. International journal of epidemiology, 46(1), 348-355.

Berry, W. (2015). The unsettling of America: Culture \& agriculture. Catapult.

Berwick, D. M. (2003). Disseminating innovations in health care. JAMA, 289(15), 19691975.

Bevans, K. B., Sanchez, B., Teneralli, R., \& Forrest, C. B. (2011). Children's eating behavior: the importance of nutrition standards for foods in schools. Journal of School Health, 81(7), 424-429.

Biglan, A., Ary, D., \& Wagenaar, A. C. (2000). The value of interrupted time-series experiments for community intervention research. Prevention Science, 1(1), 3149.

Birken, S. A., Bunger, A. C., Powell, B. J., Turner, K., Clary, A. S., Klaman, S. L., ... \& Chatham, J. R. S. (2017). Organizational theory for dissemination and implementation research. Implementation Science, 12(1), 62.

Birken, S. A., Lee, S. Y. D., Weiner, B. J., Chin, M. H., Chiu, M., \& Schaefer, C. T. (2015). From strategy to action: how top managers' support increases middle managers' commitment to innovation implementation in health care organizations. Health care management review, 40(2), 159.

Blackburn, G. L. (2011). Medicalizing obesity: individual, economic, and medical consequences. AMA Journal of Ethics, 13(12), 890-895.

Blanck, H. M., \& Collins, J. (2013). CDC's winnable battles: Improved nutrition, physical activity, and decreased obesity.

Block, J. P., Chandra, A., McManus, K. D., \& Willett, W. C. (2010). Point-of-purchase price and education intervention to reduce consumption of sugary soft drinks. American Journal of Public Health, 100(8), 1427-1433.

Blue Cross and Blue Shield Association (BCBS). (2010) Federal Policy Recommendations for Combating Childhood Obesity. Chicago: Blue Cross and Blue Shield Association.

Blumenthal, S. J., Hoffnagle, E. E., Leung, C. W., Lofink, H., Jensen, H. H., Foerster, S. B., ... \& Willett, W. C. (2014). Strategies to improve the dietary quality of Supplemental Nutrition Assistance Program (SNAP) beneficiaries: an assessment of stakeholder opinions. Public health nutrition, 17(12), 2824-2833.

Bodor, J. N., Rose, D., Farley, T. A., Swalm, C., \& Scott, S. K. (2008). Neighbourhood fruit and vegetable availability and consumption: the role of small food stores in an urban environment. Public health nutrition, 11(4), 413-420.

Boles, M., Adams, A., Gredler, A., \& Manhas, S. (2014). Ability of a mass media campaign to influence knowledge, attitudes, and behaviors about sugary drinks and obesity. Preventive medicine, 67, S40-S45.

Boone, J. E., Gordon-Larsen, P., Adair, L. S., \& Popkin, B. M. (2007). Screen time and physical activity during adolescence: longitudinal effects on obesity in young adulthood. International Journal of Behavioral Nutrition and Physical Activity, 4(1), 26.

Bordenave, J. D. (1976). Communication of agricultural innovations in Latin America: The need for new models. Communication research, 3(2), 135-154. 
Bosch, M., Van Der Weijden, T., Wensing, M., \& Grol, R. (2007). Tailoring quality improvement interventions to identified barriers: a multiple case analysis. Journal of evaluation in clinical practice, 13(2), 161-168.

Boston Public Health Commission (BPHC). 2016. Boston Public Health Commission, Healthy Beverages in Hospitals Campaign. Retrieved 28 May 2019 from: http://www.bphc.org/whatwedo/healthy-eating-active-living/healthybeverages/Documents/HospitalHealthyBeverageActivitiesSummary.pdf.

Bourgeois III, L. J. (1981). On the measurement of organizational slack. Academy of Management review, 6(1), 29-39.

Boyland, E. J., \& Harris, J. L. (2017). Regulation of food marketing to children: are statutory or industry self-governed systems effective?. Public health nutrition, 20(5), 761-764.

Bradley, E. H., Webster, T. R., Baker, D., Schlesinger, M., Inouye, S. K., Barth, M. C., ... \& Koren, M. J. (2004). Translating research into practice: speeding the adoption of innovative health care programs. Issue Brief. Commonwealth Fund, 724(1), 12.

Brambila-Macias, J., Shankar, B., Capacci, S., Mazzocchi, M., Perez-Cueto, F. J., Verbeke, W., \& Traill, W. B. (2011). Policy interventions to promote healthy eating: a review of what works, what does not, and what is promising. Food and nutrition bulletin, 32(4), 365-375.

Brammer, S., \& Millington, A. (2003). The effect of stakeholder preferences, organizational structure and industry type on corporate community involvement. Journal of Business ethics, 45(3), 213-226.

Brantley, S. L. (2009). Implementation of the enteral nutrition practice recommendations. Nutrition in clinical practice, 24(3), 335-343.

Bray, G. A., \& Popkin, B. M. (1998). Dietary fat intake does affect obesity!. The American journal of clinical nutrition, 68(6), 1157-1173.

Brown, D. M., \& Tammineni, S. K. (2009). Managing sales of beverages in schools to preserve profits and improve children's nutrition intake in 15 Mississippi schools. Journal of the American Dietetic Association, 109(12), 2036-2042.

Brownell, K. D., \& Frieden, T. R. (2009). Ounces of prevention-the public policy case for taxes on sugared beverages. New England Journal of Medicine, 360(18), 1805-1808.

Brownell, K. D., Kersh, R., Ludwig, D. S., Post, R. C., Puhl, R. M., Schwartz, M. B., \& Willett, W. C. (2010). Personal responsibility and obesity: a constructive approach to a controversial issue. Health affairs, 29(3), 379-387.

Brownell, K. D., \& Ludwig, D. S. (2011). The Supplemental Nutrition Assistance Program, soda, and USDA policy: who benefits?. Jama, 306(12), 1370-1371.

Bruss, M. B., Morris, J., \& Dannison, L. (2003). Prevention of childhood obesity: sociocultural and familial factors. Journal of the American Dietetic Association, 103(8), 1042-1045.

Cairney, P., \& Heikkila, T. (2014). Chapter 10: A comparison of theories of the policy process. Theories of the policy process, 3. New York: Avalon Publishing.

Campos, D. (2011). Jump Start Health!: Practical Ideas to Promote Wellness in Kids of 
All Ages. Teachers College Press.

Caprio, S., Daniels, S. R., Drewnowski, A., Kaufman, F. R., Palinkas, L. A., Rosenbloom, A. L., \& Schwimmer, J. B. (2008). Influence of race, ethnicity, and culture on childhood obesity: implications for prevention and treatment: a consensus statement of Shaping America's Health and the Obesity Society. Diabetes care, 31(11), 2211-2221.

Carbone, E. T., \& Zoellner, J. M. (2012). Nutrition and health literacy: a systematic review to inform nutrition research and practice. Journal of the Academy of Nutrition and Dietetics, 112(2), 254-265.

Carter, F. A., \& Jansen, A. (2012). Improving psychological treatment for obesity. Which eating behaviours should we target?. Appetite, 58(3), 1063-1069.

Caspi, C. E., Sorensen, G., Subramanian, S. V., \& Kawachi, I. (2012). The local food environment and diet: a systematic review. Health \& place, 18(5), 1172-1187.

Centers for Disease Control and Prevention (CDC). (2003). Self-reported concern about food security associated with obesity--Washington, 1995-1999. Morbidity and mortality weekly report, 52(35), 840.

Centers for Disease Control and Prevention (CDC). (2018). Healthy Hospital Practice to Practice Series. Division of Nutrition, Physical Activity, and Obesity. Retrieved May 52019 from: https://www.cdc.gov/obesity/strategies/hospital_p2p.html

Centers for Disease Control and Prevention (CDC). (2019). Healthy Food Environments. Division of Nutrition, Physical Activity, and Obesity. Retrieved May 52019 from: https://www.cdc.gov/obesity/strategies/healthy-food-env.html

Center for Weight and Health (CWH), University of California, Berkeley. (2005). Pilot Implementation of SB 19 in California Middle and High Schools: Report on Accomplishments, Impact, and Lessons Learned. Berkeley, Calif: Center for Weight and Health, University of California, Berkeley.

Champ, C. E., Iarrobino, N. A., \& Haskins, C. P. (2019). Hospitals lead by poor example: An assessment of snacks, soda, and junk food availability in Veterans Affairs hospitals. Nutrition, 60, 70-73.

Chan, Y. E. (2000). IT value: The great divide between qualitative and quantitative and individual and organizational measures. Journal of Management Information Systems, 16(4), 225-261.

Chandon P, Wansink B. Is obesity caused by calorie underestimation? A psychophysical model of meal size estimation. Journal of Marketing Research. 2007 Feb 1;44(1):84-99.

Chang, V. W., \& Christakis, N. A. (2002). Medical modelling of obesity: a transition from action to experience in a 20th century American medical textbook. Sociology of Health \& Illness, 24(2), 151-177.

Charmaz, K. (2008). Grounded theory as an emergent method. Handbook of emergent methods, 155, 172.

Childhood Obesity Prevention Coalition (COPC). 2014. Childhood Obesity Prevention Coalition, Healthier Beverage Environments. Retrieved 20 May 2019 from: http://copcwa.org/healthier-beverage-environments/.

Chen, E., \& Bodenheimer, T. (2008). Applying the chronic care model to the 
management of obesity. Obesity Management, 4(5), 227-231.

Choquet, H., \& Meyre, D. (2011). Genetics of obesity: what have we learned?. Current genomics, 12(3), 169-179.

Chriqui, J. F., Eidson, S. S., Bates, H., Kowalczyk, S., \& Chaloupka, F. J. (2008). State sales tax rates for soft drinks and snacks sold through grocery stores and vending machines, 2007. Journal of public health policy, 29(2), 226-249.

Cloke, J. (2016). Food Security and Food Waste. Peter Jackson·Walter EL Spiess, 99.

Cohen, J. F., Gorski, M. T., Hoffman, J. A., Rosenfeld, L., Chaffee, R., Smith, L., ... \& Rimm, E. B. (2016). Healthier standards for school meals and snacks: impact on school food revenues and lunch participation rates. American journal of preventive medicine, 51(4), 485-492.

Cohen, A. K., Rai, M., Rehkopf, D. H., \& Abrams, B. (2013). Educational attainment and obesity: a systematic review. Obesity Reviews, 14(12), 989-1005.

Coiera, E. (2011). Why system inertia makes health reform so difficult. BMJ, 342, d3693.

Cornelsen, L., \& Carreido, A. (2015). Health-related taxes on foods and beverages. Food Research Collaboration, 8.

Corvalan, C., Reyes, M., Garmendia, M. L., \& Uauy, R. (2019). Structural responses to the obesity and non-communicable diseases epidemic: Update on the Chilean law of food labelling and advertising. Obesity Reviews, 20(3), 367-374.

Cradock, A. L., Kenney, E. L., McHugh, A., Conley, L., Mozaffarian, R. S., Reiner, J. F., \& Gortmaker, S. L. (2015). Peer Reviewed: Evaluating the Impact of the Healthy Beverage Executive Order for City Agencies in Boston, Massachusetts, 20112013. Preventing chronic disease, 12.

Cram, P., Nallamothu, B. K., Fendrick, A. M., \& Saint, S. (2002). Fast food franchises in hospitals. JAMA, 287(22), 2945-2946.

Creatore, M. I., Glazier, R. H., Moineddin, R., Fazli, G. S., Johns, A., Gozdyra, P., ... \& Booth, G. L. (2016). Association of neighborhood walkability with change in overweight, obesity, and diabetes. Jama, 315(20), 2211-2220.

Creswell, J. W., \& Creswell, J. D. (2017). Research design: Qualitative, quantitative, and mixed methods approaches. Sage publications.

Cusick, C. (2011). Menu-Labeling Laws: A Move from Local to National Regulation. Santa Clara L. Rev., 51, 989.

Cutter, C., Lou, D., Donze Black, J., et al. (2009). Child Nutrition Programs: Federal Options and Opportunities. Center to Prevent Childhood Obesity, Little Rock: Robert Wood Johnson Foundation.

Cyert, R. M., \& March, J. G. (1963). A Behavioural Theory of the Firm. Prentice Hall New Jersey.

Daft, R. L., \& Becker, S. W. (1980). Managerial, institutional, and technical influences on administration: A longitudinal analysis. Social Forces, 59(2), 392-413.

Damanpour, F. (1992). Organizational size and innovation. Organization studies, 13(3), $375-402$.

Datz, T. (2011). Harvard serves up its own "plate.”. Harvard Gazette, 14.

De Boo, H. A., \& Harding, J. E. (2006). The developmental origins of adult disease 
(Barker) hypothesis. Australian and New Zealand Journal of Obstetrics and Gynaecology, 46(1), 4-14.

De Ridder, D., De Vet, E., Stok, M., Adriaanse, M., \& De Wit, J. (2013). Obesity, overconsumption and self-regulation failure: The unsung role of eating appropriateness standards. Health Psychology Review, 7(2), 146-165.

Dee, A., Kearns, K., O’Neill, C., Sharp, L., Staines, A., O’Dwyer, V., ... \& Perry, I. J. (2014). The direct and indirect costs of both overweight and obesity: a systematic review. BMC Research Notes, 7(1), 242.

DiMaggio, P. J., \& Powell, W. W. (1983). The iron cage revisited: Institutional isomorphism and collective rationality in organizational fields. American sociological review, 147-160.

Ding, D., \& Gebel, K. (2012). Built environment, physical activity, and obesity: what have we learned from reviewing the literature?. Health \& place, 18(1), 100-105.

Dinour, L. M., Bergen, D., \& Yeh, M. C. (2007). The food insecurity-obesity paradox: a review of the literature and the role food stamps may play. Journal of the American Dietetic Association, 107(11), 1952-1961.

Drewnowski, A., \& Specter, S. E. (2004). Poverty and obesity: the role of energy density and energy costs. American Journal of Clinical Nutrition, 79(1), 6-16.

Drope, J., Liber, A. C., Cahn, Z., Stoklosa, M., Kennedy, R., Douglas, C. E., ... \& Drope, J. (2018). Who's still smoking? Disparities in adult cigarette smoking prevalence in the United States. CA: a cancer journal for clinicians, 68(2), 106-115.

Durden, E. D., Huse, D., Ben-Joseph, R., \& Chu, B. C. (2008). Economic costs of obesity to self-insured employers. Journal of occupational and environmental medicine, 50(9), 991-997.

Edwards, C. (2018). Agricultural subsidies. Downsizing Federal Government. Retrieved Nov 12018 from: https://www.downsizinggovernment.org/agriculture/subsidies.

Elliott-Green, A., Hyseni, L., Lloyd-Williams, F., Bromley, H., \& Capewell, S. (2016). Sugar-sweetened beverages coverage in the British media: an analysis of public health advocacy versus pro-industry messaging. BMJ open, 6(7), e011295.

Eneli, I. U., Norwood, V., Hampl, S., Ferris, M., Hibbeln, T., Patterson, K., ... \& Hassink, S. (2011). Perspectives on obesity programs at children's hospitals: insights from senior program administrators. Pediatrics, 128(Supplement 2), S86-S90.

Eneli, I. U., Oza-Frank, R., Grover, K., Miller, R., \& Kelleher, K. (2014). Instituting a sugar-sweetened beverage ban: experience from a children's hospital. American journal of public health, 104(10), 1822-1825.

Eng, M. (2012). Chicago hospital group to drop sugar-sweetened drinks. Chicago Tribune. Retrieved 21 July 2019 from: http://heartofillinois.blogspot.com/2012/04/chicago- hospital-group-to-dropsugar.html

Engelhard, C. L., Garson, A., \& Dorn, S. (2009). Reducing obesity: policy strategies from the tobacco wars. Methodist Debakey Cardiovasc J, 5(4), 46-50.

Epel, E. S., Hartman, A., Jacobs, L. M., Leung, C., Cohn, M. A., Jensen, L., ... \& 
Stanhope, K. L. (2020). Association of a workplace sales ban on sugar-sweetened beverages with employee consumption of sugar-sweetened beverages and health. JAMA Internal Medicine, 180(1), 9-16.

Estabrook, B., Zapka, J., \& Lemon, S. C. (2012). Evaluating the implementation of a hospital work-site obesity prevention intervention: applying the RE-AIM framework. Health promotion practice, 13(2), 190-197.

Ethicon. (2018). Obesity Counseling Reimbursement Fact Sheet. Retrieved Nov 22018 from: https://www.ethicon.com/na/system/files/2018-03/0874941802072018 Obesity_Rmbrsmnt_Fact_Sheet_r8\%20\%281\%29.pdf.

Evan, W. M. (1965). Superior-subordinate conflict in research organizations. Administrative Science Quarterly, 52-64.

Fairholm, M. R. (2009). Leadership and Organizational Strategy. Innovation Journal, 14(1). Farrell, P., Thow, A. M., Abimbola, S., Faruqui, N., \& Negin, J. (2017). How food insecurity could lead to obesity in LMICs: When not enough is too much: a realist review of how food insecurity could lead to obesity in low-and middle-income countries. Health promotion international, 33(5), 812-826.

Farley, T. A., Halper, H. S., Carlin, A. M., Emmerson, K. M., Foster, K. N., \& Fertig, A. R. (2017). Mass media campaign to reduce consumption of sugar-sweetened beverages in a rural area of the United States. American journal of public health, 107(6), 989-995.

Farrell, L. C., Warin, M. J., Moore, V. M., \& Street, J. M. (2016). Emotion in obesity discourse: understanding public attitudes towards regulations for obesity prevention. Sociology of health \& illness, 38(4), 543-558.

Fernandes, A. C., Oliveira, R. C., Proença, R. P., Curioni, C. C., Rodrigues, V. M., \& Fiates, G. M. (2016). Influence of menu labeling on food choices in real-life settings: a systematic review. Nutrition reviews, 74(8), 534-548.

Fernandez, S., \& Rainey, H. G. (2017). Managing successful organizational change in the public sector. In Debating Public Administration (pp. 7-26). Routledge.

Finkelstein, E. A., daCosta DiBonaventura, M., Burgess, S. M., \& Hale, B. C. (2010). The costs of obesity in the workplace. Journal of Occupational and Environmental Medicine, 52(10), 971-976.

Finkelstein, E. A., Khavjou, O. A., Thompson, H., Trogdon, J. G., Pan, L., Sherry, B., \& Dietz, W. (2012). Obesity and severe obesity forecasts through 2030. American journal of preventive medicine, 42(6), 563-570.

Flegal, K. M., Kruszon-Moran, D., Carroll, M. D., Fryar, C. D., \& Ogden, C. L. (2016). Trends in obesity among adults in the United States, 2005 to 2014. Jama, 315(21), 2284-2291.

Fontaine, K. R., Redden, D. T., Wang, C., Westfall, A. O., \& Allison, D. B. (2003). Years of life lost due to obesity. Jama, 289(2), 187-193.

Forshee, R. A., Anderson, P. A., \& Storey, M. L. (2008). Sugar-sweetened beverages and body mass index in children and adolescents: a meta-analysis. The American journal of clinical nutrition, 87(6), 1662-1671.

Foss, N. J., Husted, K., Michailova, S., \& Pedersen, T. (2003). Governing knowledge 
processes: theoretical foundations and research opportunities. Copenhagen Business School.

Foster, R. D., \& Akdere, M. (2007). Effective organizational vision: implications for human resource development. Journal of European Industrial Training, 31(2), 100-111.

Frank, L. D., Andresen, M. A., \& Schmid, T. L. (2004). Obesity relationships with community design, physical activity, and time spent in cars. American journal of preventive medicine, 27(2), 87-96.

Franklin, D. (2010). Vanderbilt Med Center Dropping McDonald's, Welcoming Au Bon Pain. Nashville Science. Retrieved 18 Oct 2018 from: https://www.nashvillescene.com/music/article/13035394/vanderbilt-med-centerdropping-mcdonalds-welcoming-au-bon-pain

Frankston, J. (2003). Health pros link sprawl with spread suburbs, obesity stir debate. The Atlanta Journal-Constitution, F1.

Freedhoff, Y., \& Stevenson, R. (2008). Frying up hospital cafeteria food. Can Med Assoc $J, 179(3), 213$.

Freeman, A. (2007). Fast food: Oppression through poor nutrition. Calif. L. Rev., 95, 2221.

French, S. A., Jeffery, R. W., Story, M., Breitlow, K. K., Baxter, J. S., Hannan, P., \& Snyder, M. P. (2001). Pricing and promotion effects on low-fat vending snack purchases: the CHIPS Study. American journal of public health, 91(1), 112.

French, S. A., Story, M., Fulkerson, J. A., \& Hannan, P. (2004). An environmental intervention to promote lower-fat food choices in secondary schools: outcomes of the TACOS Study. American journal of public health, 94(9), 1507-1512.

Frickel, S., \& Moore, K. (Eds.). (2006). The new political sociology of science: Institutions, networks, and power. Univ of Wisconsin Press.

Frieden, T. R., Dietz, W., \& Collins, J. (2010). Reducing childhood obesity through policy change: acting now to prevent obesity. Health affairs, 29(3), 357-363.

Friedman, B. (2012). Hospitals should now ban the sale of any beverages with added sugar. Lab Soft News. Retrieved 18 Sept 2019 from:

https://labsoftnews.typepad.com/lab_soft_news/2012/11/all-hospitals-should-banthe-sale-of-any-drinks-with-sugar.html

Friedman, R. (2010). Strategies to Prevent Overweight and Obesity. Yale Rudd Center for Food Policy and Obesity. New Haven, CN.

Fryar CD, Carroll MD, Ogden CL. Prevalence of overweight, obesity, and severe obesity among adults aged 20 and over: United States, 1960-1962 through 2015-2016. NCHS Health E-Stats.

Fung, C., McIsaac, J. L. D., Kuhle, S., Kirk, S. F., \& Veugelers, P. J. (2013). The impact of a population-level school food and nutrition policy on dietary intake and body weights of Canadian children. Preventive medicine, 57(6), 934-940.

Funk, L. M., Jolles, S. A., \& Voils, C. I. (2016). Obesity as a disease: has the AMA resolution had an impact on how physicians view obesity?. Surgery for Obesity and Related Diseases, 12(7), 1431-1435.

Gabel, J. R., Whitmore, H., Pickreign, J., Ferguson, C. C., Jain, A., Kc, S., \& Scherer, H. 
(2009). Obesity and the workplace: current programs and attitudes among employers and employees. Health Affairs, 28(1), 46-56.

Gardner, C. D., Whitsel, L. P., Thorndike, A. N., Marrow, M. W., Otten, J. J., Foster, G. D., ... \& Johnson, R. K. (2014). Food-and-beverage environment and procurement policies for healthier work environments. Nutrition reviews, 72(6), 390-410.

Garfinkel-Castro, A., Kim, K., Hamidi, S., \& Ewing, R. (2017). Obesity and the built environment at different urban scales: examining the literature. Nutrition reviews, 75(suppl_1), 51-61.

Gase, L. N., Kuo, T., Dunet, D. O., \& Simon, P. A. (2011). Facilitators and barriers to implementing a local policy to reduce sodium consumption in the County of Los Angeles government, California. Preventing chronic disease, 8(2), A33-A33.

Geaney, F., Kelly, C., Di Marrazzo, J. S., Harrington, J. M., Fitzgerald, A. P., Greiner, B. A., \& Perry, I. J. (2016). The effect of complex workplace dietary interventions on employees' dietary intakes, nutrition knowledge and health status: a cluster controlled trial. Preventive medicine, 89, 76-83.

Ghemawat, P., \& Ricart Costa, J. E. I. (1993). The organizational tension between static and dynamic efficiency. Strategic management journal, 14(S2), 59-73.

Glisson, C., \& Schoenwald, S. K. (2005). The ARC organizational and community intervention strategy for implementing evidence-based children's mental health treatments. Mental Health Services Research, 7(4), 243-259.

Goldman, E. F., \& Swayze, S. (2012). In-depth interviewing with health care corporate elites: Strategies for entry and engagement. International Journal of Qualitative Methods, 11(3), 230-243.

Gordon-Larsen, P., Nelson, M. C., Page, P., \& Popkin, B. M. (2006). Inequality in the built environment underlies key health disparities in physical activity and obesity. Pediatrics, 117(2), 417-424.

Grech, A., \& Allman-Farinelli, M. (2015). A systematic literature review of nutrition interventions in vending machines that encourage consumers to make healthier choices. Obesity reviews, 16(12), 1030-1041.

Greenhalgh, T., Robert, G., Macfarlane, F., Bate, P., \& Kyriakidou, O. (2004). Diffusion of innovations in service organizations: systematic review and recommendations. The Milbank Quarterly, 82(4), 581-629.

Greenhalgh, T., Wherton, J., Papoutsi, C., Lynch, J., Hughes, G., Hinder, S., ... \& Shaw, S. (2017). Beyond adoption: a new framework for theorizing and evaluating nonadoption, abandonment, and challenges to the scale-up, spread, and sustainability of health and care technologies. Journal of medical Internet research, 19(11), e367.

Gregori, D., Gafare, C. E., \& Lorenzoni, G. (2017). Does the Recent Chilean Regulation on Food Labelling Improve Chilean Population Knowledge on Nutritional Facts? A longitudinal comparison between 2012 and 2016. The FASEB Journal, 31(1_supplement), 30-7.

Gregson, J., Foerster, S. B., Orr, R., Jones, L., Benedict, J., Clarke, B., ... \& Zotz, K. (2001). System, environmental, and policy changes: using the social-ecological model as a framework for evaluating nutrition education and social marketing 
programs with low-income audiences. Journal of Nutrition Education, 33, S4S15.

Grow, H. M. G., Cook, A. J., Arterburn, D. E., Saelens, B. E., Drewnowski, A., \& Lozano, P. (2010). Child obesity associated with social disadvantage of children's neighborhoods. Social science \& medicine, 71(3), 584-591.

Gulati, R. (1999). Network location and learning: The influence of network resources and firm capabilities on alliance formation. Strategic management journal, 20(5), 397420.

Hackett, J., \& Rhoads, A. (2010) Multnomah Food Initiative Background Report. 2010 Multnomah Food Initiative. Portland, OR.

Hales, C. M., Carroll, M. D., Fryar, C. D., \& Ogden, C. L. (2017). Prevalence of obesity among adults and youth: United States, 2015-2016. Centers for Disease Control and Prevention.

Hall, K. D. (2018). Did the food environment cause the obesity epidemic?. Obesity, 26(1), 11-13.

Hall, K., \& De Lannoy, A. (2015). Children's access to education. ChildGauge.

Hannan, M. T., \& Freeman, J. (1984). Structural inertia and organizational change. American sociological review, 149-164.

Hartigan, P., Patton-Ku, D., Fidler, C., \& Boutelle, K. N. (2017). Rethink your drink: Reducing sugar-sweetened beverage sales in a children's hospital. Health promotion practice, 18(2), 238-244.

Hastings, J. S., Kessler, R. E., \& Shapiro, J. M. (2019). The Effect of SNAP on the Composition of Purchased Foods: Evidence and Implications (No. w25953). National Bureau of Economic Research.

Hawkes, C. (2006). Uneven dietary development: linking the policies and processes of globalization with the nutrition transition, obesity and diet-related chronic diseases. Globalization and health, 2(1), 4.

Health Care Without Harm (HCWH). 2014. Health Care Without Harm, Healthier Food, Healthier Hospitals Initiative. Retrieved 18 June 2019 from: http://healthierhospitals.org/challenges/healthier-food.

Healthier Hospitals Initiative (HHI). (2013). About Us. Retrieved Nov 282018 from: http://www.healthierhospitals.org/about-hh.

Heinen, L., \& Darling, H. (2009). Addressing obesity in the workplace: the role of employers. The Milbank Quarterly, 87(1), 101-122.

Henning, R., Warren, N., Robertson, M., Faghri, P., Cherniack, M., \& CPH-NEW Research Team. (2009). Workplace health protection and promotion through participatory ergonomics: an integrated approach. Public Health Reports, 124(4_supp11), 26-35.

Hill, C. J., \& Lynn, L. E. (2004). Is hierarchical governance in decline? Evidence from empirical research. Journal of Public Administration Research and Theory, 15(2), 173-195.

Hill, D., Swinburn, B., Johnson, G., Harper, T. (2010). Comprehensive Review of Food Labelling Law and Policy: Second submission from the Obesity Policy Coalition. Carlton, Victoria, Australia: Obesity Policy Coalition. Retrieved May 162019 
from:http://www.opc.org.au/downloads/submissions/food_labelling_law_and_pol icy_review_second_submission.pdf

HMO offers Weight Watchers to West Virginia Medicaid enrollees (2007). Retrieved Oct 152018 from: http:www.ama-assn.orgamednews20070219gvsd0219.htm.

Hofmann, B. (2016). Obesity as a socially defined disease: philosophical considerations and implications for policy and care. Health care analysis, 24(1), 86-100.

Hollands, G. J., Cartwright, E., Pilling, M., Pechey, R., Vasiljevic, M., Jebb, S. A., \& Marteau, T. M. (2018). Impact of reducing portion sizes in worksite cafeterias: a stepped wedge randomised controlled pilot trial. International Journal of Behavioral Nutrition and Physical Activity, 15(1), 78.

Hollingsworth, B. (2008). The measurement of efficiency and productivity of health care delivery. Health economics, 17(10), 1107-1128.

Hospital Healthy Food Initiative (HHFI). 2014. Partnership for a Healthier America, Hospital Healthy Food Initiative. Retrieved 12 July 2019 from: http://ahealthieramerica.org/our-partners/hospitals/.

Hu, F. B. (2013). Resolved: there is sufficient scientific evidence that decreasing sugarsweetened beverage consumption will reduce the prevalence of obesity and obesity-related diseases. Obesity reviews, 14(8), 606-619.

Huang, T. T., Drewnowski, A., Kumanyika, S. K., \& Glass, T. A. (2009). A systemsoriented multilevel framework for addressing obesity in the 21 st century. Preventing chronic disease, 6(3).

Huang, T. T., \& Glass, T. A. (2008). Transforming research strategies for understanding and preventing obesity. JAMA, 300(15), 1811-1813.

Hunton, A. (2018). Penn Medicine's Commitment to Encouraging Healthy Lifestyles for Patients and Staff Now Extends to Food and Beverages. Penn Medicine News.

Retrieved 20 Sept 2019 from: https://www.pennmedicine.org/news/newsreleases/2018/august/penn-medicines-commitment-to-encouraging-healthylifestyles-for-patients-and-staff

Hussey, P. S., Schneider, E. C., Rudin, R. S., Fox, D. S., Lai, J., \& Pollack, C. E. (2014). Continuity and the costs of care for chronic disease. JAMA internal medicine, 174(5), 742-748.

Institute for a Sustainable Future (ISF). 2014. Institute For a Sustainable Future, Commons Health Hospital Challenge. Retrieved 20 June 2019 from: http://www.commonshealthchallenge.org/.

Institute of Medicine (US). Committee on Assuring the Health of the Public in the 21st Century. (2003). The Future of the Public's Health in the 21st Century. National Academy Press.

International Network for Food and Obesity Research, Monitoring and Action Support (INFORMAS). (2014). Towards global benchmarking of food environments and policies to reduce obesity and diet-related non-communicable diseases: design and methods for nation-wide surveys. BMJ open, 4(5).

Isoldi, K. K., Dalton, S., Rodriguez, D. P., \& Nestle, M. (2012). Classroom "cupcake" celebrations: observations of foods offered and consumed. Journal of nutrition education and behavior, 44(1), 71-75. 
Ivankova, N. V., Creswell, J. W., \& Plano Clark, V. L. (2007). Foundations and approaches to mixed methods research. First steps in research. Pretoria: Van Schaik, 253-282.

Jaacks, L. M., Vandevijvere, S., Pan, A., McGowan, C. J., Wallace, C., Imamura, F., ... \& Ezzati, M. (2019). The obesity transition: stages of the global epidemic. The Lancet Diabetes \& Endocrinology, 7(3): 231-240.

Jabs J, Devine CM. Time scarcity and food choices: an overview. Appetite. 2006;47(2):196-204.

Jaime, P. C., \& Lock, K. (2009). Do school based food and nutrition policies improve diet and reduce obesity?. Preventive medicine, 48(1), 45-53.

Jannah, N., Hild, J., Gallagher, C., \& Dietz, W. (2018). Coverage for Obesity Prevention and Treatment Services: Analysis of Medicaid and State Employee Health Insurance Programs. Obesity, 26(12), 1834-1840.

Jaskiewicz, L., Dombrowski, R. D., Drummond, H. M., Barnett, G. M., Mason, M., \& Welter, C. (2013). Peer Reviewed: Partnering With Community Institutions to Increase Access to Healthful Foods Across Municipalities. Preventing chronic disease, 10.

Jevitt, C. M. (2019). Obesity and Socioeconomic Disparities: Rethinking Causes and Perinatal Care. The Journal of perinatal \& neonatal nursing, 33(2), 126-135.

Jia, H., \& Lubetkin, E. I. (2005). The impact of obesity on health-related quality-of-life in the general adult US population. Journal of public health, 27(2), 156-164.

Jilcott Pitts, S. B., Graham, J., Mojica, A., Stewart, L., Walter, M., Schille, C., ... \& Bradley, A. (2016). Implementing healthier foodservice guidelines in hospital and federal worksite cafeterias: barriers, facilitators and keys to success. Journal of human nutrition and dietetics, 29(6), 677-686.

Joint Commission on Accreditation of Healthcare Organizations (JCAHO). 2020. Accreditation and Certification Standards. Retrieved 1 September 2020 from: https://www.jointcommission.org/accreditation-and-certification/health-caresettings/hospital/learn/our-standards/

Jokela, M., Elovainio, M., \& Kivimäki, M. (2008). Lower fertility associated with obesity and underweight: the US National Longitudinal Survey of Youth. The American journal of clinical nutrition, 88(4), 886-893.

Jones, B. D. (2003). Bounded rationality and political science: Lessons from public administration and public policy. Journal of Public Administration Research and Theory, 13(4), 395-412.

Jones, C., Hesterly, W. S., Fladmoe-Lindquist, K., \& Borgatti, S. P. (1998). Professional service constellations: How strategies and capabilities influence collaborative stability and change. Organization Science, 9(3), 396-410.

Jordan, A., Taylor Piotrowski, J., Bleakley, A., \& Mallya, G. (2012). Developing media interventions to reduce household sugar-sweetened beverage consumption. The Annals of the American Academy of Political and Social Science, 640(1), 118135.

Judge, W. Q., \& Blocker, C. P. (2008). Organizational capacity for change and strategic 
ambidexterity: Flying the plane while rewiring it. European Journal of Marketing, 42(9/10), 915-926.

Kallio, H., Pietilä, A. M., Johnson, M., \& Kangasniemi, M. (2016). Systematic methodological review: developing a framework for a qualitative semi-structured interview guide. Journal of advanced nursing, 72(12), 2954-2965.

Kansas City Star. (2015). Children's Mercy Hospital in Missouri Eliminates Soda, Sugary Drinks. Kansas City Star. Retrieved 19 July 2019 from: https://www.beckershospitalreview.com/hospital-managementadministration/childrens-mercy-hospital-in-missouri-eliminates-soda-sugarydrinks.html

Kapstein, E. B. (2001). The corporate ethics crusade. Foreign affairs, 105-119.

Karat Gold Partnership (KGP). 2014. Karat Gold Partnership, Greater Kansas City Food Policy Coalition. Retrieved 12 July 2019 from: http://www.kcfoodpolicy.org/?q=karat-gold-partnership.

Kaspin, L. C., Gorman, K. M., \& Miller, R. M. (2013). Systematic review of employersponsored wellness strategies and their economic and health-related outcomes. Population health management, 16(1), 14-21.

Katz, D., \& Kahn, R. L. (1978). The social psychology of organizations (Vol. 2, p. 528). New York: Wiley.

Katz, D. A., McHorney, C. A., \& Atkinson, R. L. (2000). Impact of obesity on healthrelated quality of life in patients with chronic illness. Journal of general internal medicine, 15(11), 789-796.

Kaufman, L., \& Karpati, A. (2007). Understanding the sociocultural roots of childhood obesity: food practices among Latino families of Bushwick, Brooklyn. Social science \& medicine, 64(11), 2177-2188.

Kaur, M. (2016). Application of mixed method approach in public health research. Indian journal of community medicine: official publication of Indian Association of Preventive \& Social Medicine, 41(2), 93.

Kautiainen, S., Koivusilta, L., Lintonen, T., Virtanen, S. M., \& Rimpelä, A. (2005). Use of information and communication technology and prevalence of overweight and obesity among adolescents. International journal of obesity, 29(8), 925.

Keen, S., \& Todres, L. (2007). Strategies for disseminating qualitative research findings: Three exemplars. In Forum: Qualitative Social Research, 8(3), 1-17.

Keeney, D., \& Kemp, L. (2004). A new agricultural policy for the United States. Nato Science Series: V Science and Technology Policy, 41, 29-47.

Kemp, E., Bui, M., \& Grier, S. (2013). When food is more than nutrition: Understanding emotional eating and overconsumption. Journal of Consumer Behaviour, 12(3), 204-213.

Kenis, P., \& Schneider, V. (1991). Policy networks and policy analysis: scrutinizing a new analytical toolbox. In Policy networks: Empirical evidence and theoretical considerations (pp. 25-59). Campus Verlag.

Khadaee, G. H., \& Saeidi, M. (2016). Increases of Obesity and Overweight in Children: an Alarm for Parents and Policymakers. International journal of pediatrics, 4(4), 1591-1601. 
Khan, T., Powell, L. M., \& Wada, R. (2012). Fast food consumption and food prices: evidence from panel data on 5th and 8th grade children. Journal of obesity, 2012.

Kim, D. D., \& Basu, A. (2016). Estimating the medical care costs of obesity in the United States: systematic review, meta-analysis, and empirical analysis. Value in Health, 19(5), 602-613.

Kim, G. W., Lin, J. E., Blomain, E. S., \& Waldman, S. A. (2014). Antiobesity pharmacotherapy: new drugs and emerging targets. Clinical Pharmacology \& Therapeutics, 95(1), 53-66.

Kimmons, J., Jones, S., McPeak, H. H., \& Bowden, B. (2012). Developing and implementing health and sustainability guidelines for institutional food service. Advances in Nutrition, 3(3), 337-342.

Klatzkin, R. R., Gaffney, S., Cyrus, K., Bigus, E., \& Brownley, K. A. (2018). Stressinduced eating in women with binge-eating disorder and obesity. Biological psychology, 131, 96-106.

Klaczynski, P. A., Goold, K. W., Mudry, J.J. (2004). Culture, obesity stereotypes, selfesteem, and the "thin ideal": A social identity perspective. Journal of Youth and Adolescence, 33(4):307, 17.

Klein, K. (2015). Values-based food procurement in hospitals: the role of health care group purchasing organizations. Agriculture and human values, 32(4), 635-648.

Klein KJ, Ziegert JC, Knight AP, Xiao Y. Dynamic delegation: Shared, hierarchical, and deindividualized leadership in extreme action teams. Administrative science quarterly. 2006 Dec;51(4):590-621.

Koc, E. (2013). Inversionary and liminoidal consumption: Gluttony on holidays and obesity. Journal of Travel \& Tourism Marketing, 30(8), 825-838.

Kolasa, K. M., Dial, J., Gaskins, S., \& Currie, R. (2010). Moving toward healthier-eating environments in hospitals. Nutrition Today, 45(2), 54-63.

Krein, S. L., Damschroder, L. J., Kowalski, C. P., Forman, J., Hofer, T. P., \& Saint, S. (2010). The influence of organizational context on quality improvement and patient safety efforts in infection prevention: a multi-center qualitative study. Social science \& medicine, 71(9), 1692-1701.

Krein, S. L., Olmsted, R. N., Hofer, T. P., Kowalski, C., Forman, J., Banaszak-Holl, J., \& Saint, S. (2006). Translating infection prevention evidence into practice using quantitative and qualitative research. American journal of infection control, 34(8), $507-512$.

Krieger, N., \& Davey Smith, G. (2004). "Bodies count," and body counts: social epidemiology and embodying inequality. Epidemiologic reviews, 26(1), 92-103.

Krueger, J. E., Krub, K. R., \& Hayes, L. A. (2010). Planting the seeds for public health: how the Farm Bill can help farmers to produce and distribute healthy foods. Farmer's Legal Action Group. St. Paul, MN.

Kumanyika, S. (2005). Obesity, health disparities, and prevention paradigms: hard questions and hard choices. Preventing Chronic Disease, 2(4).

Kumanyika, S. (2007). Obesity prevention concepts and frameworks. In Handbook of obesity prevention (pp. 85-114). Springer, Boston, MA.

Kumanyika, S., Taylor, W. C., Grier, S. A., Lassiter, V., Lancaster, K. J., Morssink, C. 
B., \& Renzaho, A. M. (2012). Community energy balance: a framework for contextualizing cultural influences on high risk of obesity in ethnic minority populations. Preventive medicine, 55(5), 371-381.

Kyle, T. K., Dhurandhar, E. J., \& Allison, D. B. (2016). Regarding obesity as a disease: evolving policies and their implications. Endocrinology and Metabolism Clinics, 45(3), 511-520.

Lake, A., \& Townshend, T. (2006). Obesogenic environments: exploring the built and food environments. The Journal of the Royal society for the Promotion of Health, 126(6), 262-267.

Lanningham-Foster, L., Nysse, L. J., \& Levine, J. A. (2003). Labor saved, calories lost: the energetic impact of domestic labor-saving devices. Obesity research, 11(10), $1178-1181$.

Larson, N., \& Story, M. (2009). A review of environmental influences on food choices. Annals of Behavioral Medicine, 38(suppl_1), s56-s73.

Lasker, R. D., Weiss, E. S., \& Miller, R. (2001). Partnership synergy: a practical framework for studying and strengthening the collaborative advantage. The Milbank Quarterly, 79(2), 179-205.

Lawrence, T. B. (1999). Institutional strategy. Journal of management, 25(2), 161-187.

Lawrence, S., Boyle, M., Craypo, L., \& Samuels, S. (2009). The food and beverage vending environment in health care facilities participating in the healthy eating, active communities program. Pediatrics, 123(Supplement 5), S287-S292.

Lawrence, P. R., \& Lorsch, J. W. (1967). Differentiation and integration in complex organizations. Administrative science quarterly, 1-47.

Lederer, A., Toner, C., Krepp, E. M., \& Curtis, C. J. (2014). Understanding hospital cafeterias: results from cafeteria manager interviews. Journal of Public Health Management and Practice, 20, S50-S53.

Lerner, D., Rodday, A. M., Cohen, J. T., \& Rogers, W. H. (2013). A systematic review of the evidence concerning the economic impact of employee-focused health cpromotion and wellness programs. Journal of Occupational and Environmental Medicine, 55(2), 209-222.

Lessard, L., Poland, M., \& Trotter, M. (2014). Peer Reviewed: Lessons Learned From a Healthful Vending Pilot Program in Delaware State Agency Buildings, 20112012. Preventing chronic disease, 11.

Lesser, L. I., Cohen, D. A., \& Brook, R. H. (2012). Changing eating habits for the medical profession. JAMA, 308(10), 983-984.

Lesser, L. I., \& Lucan, S. C. (2013). The ethics of hospital cafeteria food. AMA Journal of Ethics, 15(4), 299-305.

Lesser, L. I., Hunnes, D. E., Reyes, P., Arab, L., Ryan, G. W., Brook, R. H., \& Cohen, D. A. (2012). Assessment of food offerings and marketing strategies in the foodservice venues at California Children's Hospitals. Academic pediatrics, 12(1), 6267.

Lev-Ram, M. (2015). Twitter's most Tweet-worthy perks. Fortune. Retrieved Nov 12018 from: http://fortune.com/2015/03/05/twitter-best-perks/.

Levine, P., Uhlir, M., \& White, J. (1961). Ah, an incomplete suppression of A resembling 
Oh. Vox sanguinis, 6(5), 561-567.

Lewin, A. Y., Long, C. P., \& Carroll, T. N. (1999). The coevolution of new organizational forms. Organization science, 10(5), 535-550.

Lindblom, C. E. (1979). Still muddling, not yet through. Public Administration Review. $39,517-26$.

Liu, P. J., Wisdom, J., Roberto, C. A., Liu, L. J., \& Ubel, P. A. (2014). Using behavioral economics to design more effective food policies to address obesity. Applied Economic Perspectives and Policy, 36(1), 6-24.

Lobstein, T., Jackson-Leach, R., Moodie, M. L., Hall, K. D., Gortmaker, S. L., Swinburn, B. A., ... \& McPherson, K. (2015). Child and adolescent obesity: part of a bigger picture. The Lancet, 385(9986), 2510-2520.

Lohman, B. J., Stewart, S., Gundersen, C., Garasky, S., \& Eisenmann, J. C. (2009). Adolescent overweight and obesity: links to food insecurity and individual, maternal, and family stressors. Journal of Adolescent Health, 45(3), 230-237.

Long, M. W., Henderson, K. E., \& Schwartz, M. B. (2010). Evaluating the impact of a Connecticut program to reduce availability of unhealthy competitive food in schools. Journal of School Health, 80(10), 478-486.

Long, M. W., Luedicke, J., Dorsey, M., Fiore, S. S., \& Henderson, K. E. (2013). Impact of Connecticut legislation incentivizing elimination of unhealthy competitive foods on National School Lunch Program participation. American journal of public health, 103(7), e59-e66.

Lopez, R. (2004). Urban sprawl and risk for being overweight or obese. American journal of public health, 94(9), 1574-1579.

Lord, R. G., \& Maher, K. J. (2002). Leadership and information processing: Linking perceptions and performance. Routledge.

Lucas, A., Fewtrell, M. S., \& Cole, T. J. (1999). Fetal origins of adult disease- the hypothesis revisited. Bmj, 319(7204), 245-249.

Lubell, M., Mewhirter, J. M., Berardo, R., \& Scholz, J. T. (2017). Transaction costs and the perceived effectiveness of complex institutional systems. Public Administration Review, 77(5), 668-680.

Lucero, J., Wallerstein, N., Duran, B., Alegria, M., Greene-Moton, E., Israel, B., ... \& Schulz, A. (2018). Development of a mixed methods investigation of process and outcomes of community-based participatory research. Journal of Mixed Methods Research, 12(1), 55-74.

Ludwig, D. S. (2018). Epidemic childhood obesity: not yet the end of the beginning. Pediatrics, 141(3), e20174078.

Mackenbach, J. D., Rutter, H., Compernolle, S., Glonti, K., Oppert, J. M., Charreire, H., ... \& Lakerveld, J. (2014). Obesogenic environments: a systematic review of the association between the physical environment and adult weight status, the SPOTLIGHT project. BMC public health, 14(1), 233.

Malhotra, A. (2013). It's time to ban junk food on hospital premises. BMJ, 346, f3932.

Malik, V. S., Schulze, M. B., \& Hu, F. B. (2006). Intake of sugar-sweetened beverages and weight gain: a systematic review. The American journal of clinical nutrition, 84(2), 274-288. 
Mason, M. (2014). Working with community partners to implement and evaluate the Chicago Park District's 100\% Healthier Snack Vending Initiative. Preventing chronic disease, 11.

Masse, L. C., Naiman, D., \& Naylor, P. J. (2013). From policy to practice: implementation of physical activity and food policies in schools. International Journal of Behavioral Nutrition and Physical Activity, 10(1), 71.

Mather, A. A., Cox, B. J., Enns, M. W., \& Sareen, J. (2009). Associations of obesity with psychiatric disorders and suicidal behaviors in a nationally representative sample. Journal of psychosomatic research, 66(4), 277-285.

Mathew, H., Paschou, S. A., Aramapatzi, K. M., Hsu, W., \& Mantzoros, C. S. (2017). Obesity: Genetics, Pathogenesis, Therapy. Principles of Diabetes Mellitus, 1-17.

March, J. G. (1991). How decisions happen in organizations. Human-computer interaction, 6(2), 95-117.

March, J. G., \& Cyert, R. M. (1963). A behavioral theory of the firm (Vol. 43). Englewood Cliffs, NJ: Prentice-Hall.

March, J. G., \& Olsen, J. P. (1975). The uncertainty of the past: Organizational learning under ambiguity. European journal of political research, 3(2), 147-171.

March, J. G., \& Olsen, J. P. (1983). The new institutionalism: Organizational factors in political life. American political science review, 78(3), 734-749.

March, J. G., \& Simon, H. A. (1993). Organizations. 1958. NY: Wiley, New York.

Marshall, A. P., Cahill, N. E., Gramlich, L., MacDonald, G., Alberda, C., \& Heyland, D. K. (2012). Optimizing nutrition in intensive care units: empowering critical care nurses to be effective agents of change. American Journal of Critical Care, 21(3), 186-194.

May, C., Finch, T., Mair, F., Ballini, L., Dowrick, C., Eccles, M., ... \& Rogers, A. (2007). Understanding the implementation of complex interventions in health care: the normalization process model. BMC health services research, 7(1), 148.

May, C. R., Johnson, M., \& Finch, T. (2016). Implementation, context and complexity. Implementation Science, 11(1), 141.

McCormick, B., Stone, I., \& Corporate Analytical Team. (2007). Economic costs of obesity and the case for government intervention. Obesity reviews, 8, 161-164.

McDonald, C. M., Karamlou, T., Wengle, J. G., Gibson, J., \& McCrindle, B. W. (2006). Nutrition and exercise environment available to outpatients, visitors, and staff in children's hospitals in Canada and the United States. Archives of pediatrics \& adolescent medicine, 160(9), 900-905.

McGregor, J. (2018) 'We will not serve or pay for meat:' WeWork takes the green workplace to a new level. Washington Post, Jul 162018.

McGuire, S. (2012). Accelerating progress in obesity prevention: solving the weight of the nation. Institute of Medicine, Washington, DC: National Academies Press.

McSweeney, L., Spence, S., Anderson, J., Wrieden, W., \& Haighton, C. (2018). Parental perceptions of onsite hospital food outlets in a large hospital in the North East of England: A qualitative interview study. PloS one, 13(11), e0205416.

Mekonnen, T. A., Odden, M. C., Coxson, P. G., Guzman, D., Lightwood, J., Wang, Y. 
C., \& Bibbins-Domingo, K. (2013). Health benefits of reducing sugar-sweetened beverage intake in high risk populations of California: results from the cardiovascular disease (CVD) policy model. PLoS One, 8(12), e81723.

Meyer, A. D. (1982). Adapting to environmental jolts. Administrative science quarterly, $515-537$.

Meyer, J. W., \& Scott, W. R. (1992). Organizational environments: Ritual and rationality. New York: Sage Publications.

Meyer, J. W., \& Rowan, B. (1977). Institutionalized organizations: Formal structure as myth and ceremony. American Journal of Sociology, 83(2), 340-363.

MHealthy Nutrition Guidelines. (2017). Nutrition, University of Michigan. Retrieved 18 Sep 2019 from: https://hr.umich.edu/sites/default/files/CVC-Guidelines-2017.pdf

Mhurchu, C. N., Aston, L. M., \& Jebb, S. A. (2010). Effects of worksite health promotion interventions on employee diets: a systematic review. BMC public health, 10(1), 62.

Miller, D., Dröge, C., \& Toulouse, J. M. (1988). Strategic process and content as mediators between organizational context and structure. Academy of Management Journal, 31(3), 544-569.

Miller, E., \& Halberstadt, J. (2005). Media consumption, body image and thin ideals in New Zealand men and women. New Zealand Journal of Psychology, 34(3), 189195.

Moher, D., Liberati, A., Tetzlaff, J., \& Altman, D. G. (2009). Preferred reporting items for systematic reviews and meta-analyses: the PRISMA statement. Annals of internal medicine, 151(4), 264-269.

Moher, D., Schulz, K. F., Altman, D., \& Consort Group. (2001). The CONSORT statement: revised recommendations for improving the quality of reports of parallel-group randomized trials. Jama, 285(15), 1987-1991.

Mohr, L. B. (1969). Determinants of innovation in organizations. American political science review, 63(1), 111-126.

Moran, A., Krepp, E. M., Johnson, C. C., \& Lederer, A. (2016). An Intervention to Increase Availability of Healthy Foods and Beverages in New York City Hospitals: The Healthy Hospital Food Initiative, 2010-2014. Preventing chronic disease, 13, E77-E77.

Moran, A., Lederer, A., \& Curtis, C. J. (2015). Use of nutrition standards to improve nutritional quality of hospital patient meals: findings from New York City's Healthy Hospital Food Initiative. Journal of the Academy of Nutrition and Dietetics, 115(11), 1847-1854.

Morland, K., Roux, A. V. D., \& Wing, S. (2006). Supermarkets, other food stores, and obesity: the atherosclerosis risk in communities study. American journal of preventive medicine, 30(4), 333-339.

Moodie, R., Swinburn, B., Richardson, J., \& Somaini, B. (2006). Childhood obesity-a sign of commercial success, but a market failure. International Journal of Pediatric Obesity, 1(3), 133-138.

Moore, L. V., Diez Roux, A. V., Nettleton, J. A., \& Jacobs Jr, D. R. (2008). Associations 
of the local food environment with diet quality - a comparison of assessments based on surveys and geographic information systems: the multi-ethnic study of atherosclerosis. American journal of epidemiology, 167(8), 917-924.

Morrissey, E. (2012) Big Gulp? Meet Big Brother. Opinion. CNN. Retrieved 2 Feb 2020 from: https://www.cnn.com/2012/06/01/opinion/morrissey-bloombergsoda/index.html

Mozaffarian, D., Angell, S. Y., Lang, T., \& Rivera, J. A. (2018). Role of government policy in nutrition - barriers to and opportunities for healthier eating. $B M J, 361$, k2426.

Mozaffarian, D., \& Ludwig, D. S. (2011). Dietary guidelines in the 21st century-a time for food. Jama, 304(6), 681-682.

Munoz Yanez, C., García Vargas, G. G., \& Pérez-Morales, R. (2017). Monogenic, Polygenic and Multifactorial Obesity in Children: Genetic and Environmental Factors. Austin J Nutr Metab, 4(3), 1052.

Mytton, O. T., Clarke, D., \& Rayner, M. (2012). Taxing unhealthy food and drinks to improve health. Bmj, 344, e2931.

Nathan, N., Murawski, B., Hope, K., Young, S., Sutherland, R., Hodder, R., ... \& Tzelepis, F. (2020). The Efficacy of Workplace Interventions on Improving the Dietary, Physical Activity and Sleep Behaviours of School and Childcare Staff: A Systematic Review. International journal of environmental research and public health, 17(14), 4998.

National Academies of Sciences, Engineering, and Medicine (NASEM). (2017). The Challenges of Treating Obesity and Overweight: Proceedings of a Workshop. Roundtable on Obesity Solutions. Washington (DC): National Academies Press (US); 2017 Dec 21.

Neely, E., Walton, M., \& Stephens, C. (2014). Young people's food practices and social relationships. A thematic synthesis. Appetite, 82, 50-60.

Neff, R. A., Palmer, A. M., McKenzie, S. E., \& Lawrence, R. S. (2009). Food systems and public health disparities. Journal of Hunger \& Environmental Nutrition, 4(34), 282-314.

Nelson, D. R., Adger, W. N., \& Brown, K. (2007). Adaptation to environmental change: contributions of a resilience framework. Annu. Rev. Environ. Resour., 32, 395419.

Nelson, L., \& Zarracina, J. (2016). Why LaCroix sparkling water is suddenly everywhere. Vox Media. Retrieved 7 July 2020 from: https://www.vox.com/2016/6/20/11666314/lacroix-sparkling-water-seltzer.

Nestle, M. (2011). What Google's Famous Cafeterias Can Teach Us About Health. The Atlantic. Retrieved Sept 192018 from: https://www.theatlantic.com/health/archive/2011/07/what-googles-famouscafeterias-can-teach-us-about-health/241876/.

Nestle, M., \& Jacobson, M. F. (2000). Halting the obesity epidemic: a public health policy approach. Public health reports, 115(1), 12.

Nobrega, S., Champagne, C., Punnett, L., Abreu, M., \& Kernan, L. (2014). 
Understanding and counteracting the obesogenic work environment. Lowell, MA:

Center for the Promotion of Health in the New England Workplace.

Nyberg, M., \& Doktor Olsen, T. (2010). Meals at work: integrating social and architectural aspects. International Journal of Workplace Health Management, 3(3), 222-232.

O’Brien, B. C., Harris, I. B., Beckman, T. J., Reed, D. A., \& Cook, D. A. (2014). Standards for reporting qualitative research: a synthesis of recommendations. Academic Medicine, 89(9), 1245-1251.

O'Cathain, A. (2010). Assessing the quality of mixed methods research: Toward a comprehensive framework. Handbook of mixed methods in social and behavioral research, 531, 555.

Obesity Prevention Program (OPP). (2018). Texas Department of State and Health Services, Department of Health and Human Services. Retrieved Oct 192018 from: https://www.dshs.texas.gov/obesity/?terms=obesity $\% 20$ prevention.

Oregon Association of Hospitals and Health Systems (OAHHS). (2017). Economic Contributions of Oregon's Community Hospitals. Retrieved 18 Oct 2019 from: https://www.oahhs.org/assets/documents/publications/OAHHS\%202017\%20Final \%20-\%20Main\%20Report.pdf

Oregon Health Authority (OHA). (2012). Safety net clinics in Oregon: Federally qualified health centers. Oregon Health Policy and Research, 7. Retrieved 18 Oct

2019 from: https:/www.oregon.gov/oha/HPA/HP-PCO/Documents/FQHCs.pdf

Oregon Health Authority (OHA). (2015). State Health Improvement Plan: 2015-2019. Public Health Division, Oregon Healthy Authority. Retrieved Oct 12018 from: https://www.oregon.gov/oha/PH/ABOUT/Documents/ship/oregon-state-healthimprovement-plan.pdf.

Oregon Hospital Guide. (2019). Hospitals by Measure. Find a Hospital. Retrieved Oct 12 2019 from: http://oregonhospitalguide.org/hospitals-by-measure/hospitals-bymeasure.html.

Oliver, C. (1991). Strategic responses to institutional processes. Academy of management review, 16(1), 145-179.

Olstad, D. L., Raine, K. D., \& McCargar, L. J. (2012). Adopting and implementing nutrition guidelines in recreational facilities: public and private sector roles. A multiple case study. BMC public health, 12(1), 376.

Onufrak, S. J., Zaganjor, H., Moore, L. V., Carlson, S., Kimmons, J., \& Galuska, D. (2016). Nutrition Standards for Food Service Guidelines for Foods Served or Sold in Municipal Government Buildings or Worksites, United States, 2014.

Preventing chronic disease, 13, E172-E172.

Ott, J. S. (1989). The organizational culture perspective (pp. 221-243). Chicago: Dorsey Press.

Palinkas, L. A., Aarons, G. A., Horwitz, S., Chamberlain, P., Hurlburt, M., \& Landsverk, J. (2011). Mixed method designs in implementation research. Administration and Policy in Mental Health and Mental Health Services Research, 38(1), 44-53.

Palinkas, L. A. (2014). Qualitative and mixed methods in mental health services and 
implementation research. Journal of Clinical Child \& Adolescent Psychology, 43(6), 851-861.

Palmedo, P. C., \& Gordon, L. M. (2019). How to be SSB-free: Assessing the attitudes and readiness for a sugar sweetened beverage-free health care center in the Bronx, NY. PloS one, 14(5), e0215127.

Paschou, S. A., Spyrou, N., \& Mantzoros, C. S. (2016). Research advances in metabolism 2015. Metabolism-Clinical and Experimental, 65(3), 27-37.

Parekh, A. K. (2017). Public health communications: Lessons learned from the affordable care act. Am J Public Health, 107(5), 639-641.

Park, S., \& Lee, J. (2016). 'When operating a cafeteria, sales come before nutrition'finding barriers and facilitators to serving reduced-sodium meals in worksite cafeterias. Public health nutrition, 19(8), 1506-1516.

Parker, R. M., Ratzan, S. C., \& Lurie, N. (2003). Health literacy: a policy challenge for advancing high-quality health care. Health affairs, 22(4), 147-153.

Pechey, R., Cartwright, E., Pilling, M., Hollands, G. J., Vasiljevic, M., Jebb, S. A., \& Marteau, T. M. (2019). Impact of increasing the proportion of healthier foods available on energy purchased in worksite cafeterias: A stepped wedge randomized controlled pilot trial. Appetite, 133, 286-296.

Pedersen, P. U., Tewes, M., \& Bjerrum, M. (2012). Implementing nutritional guidelinesthe effect of systematic training for nurse nutrition practitioners. Scandinavian journal of caring sciences, 26(1), 178-185.

Penfold, R. B., \& Zhang, F. (2013). Use of interrupted time series analysis in evaluating health care quality improvements. Academic pediatrics, 13(6), S38-S44.

Penrose, E. (1959). The Theory of the Growth of the Firm, New York: John Wiley and Sons.

Perez-Escamilla, R., Lutter, C. K., Rabadan-Diehl, C., Rubinstein, A., Calvillo, A., Corvalán, C., ... \& Ewart-Pierce, E. (2017). Prevention of childhood obesity and food policies in Latin America: from research to practice. Obesity reviews, 18, 28-38.

Piernas, C., \& Popkin, B. M. (2009). Snacking increased among US adults between 1977 and 2006. The Journal of nutrition, 140(2), 325-332.

PLoS Medicine Editors (PLoS). (2013) Addressing the Wicked Problem of Obesity through Planning and Policies. PLoS Med 10(6): e1001475.

Plsek, P. (2003). Complexity and the adoption of innovation in health care. Accelerating Quality Improvement in Health Care: Strategies to Accelerate the Diffusion of Evidence-Based Innovations. Washington, DC: National Institute for Health care Management Foundation and National Committee for Quality in Health Care.

Pluye, P., \& Hong, Q. N. (2014). Combining the power of stories and the power of numbers: mixed methods research and mixed studies reviews. Annual review of public health, 35, 29-45.

Pomeranz, J. L. (2011). The unique authority of state and local health departments to address obesity. American Journal of Public Health, 101(7), 1192-1197.

Pomeranz, J. L., Mozaffarian, D., \& Micha, R. (2017). The potential for federal 
preemption of state and local sugar-sweetened beverage taxes. American journal of preventive medicine, 53(5), 740-743.

Pomeranz, J. L., Zellers, L., Bare, M., \& Pertschuk, M. (2019). State Preemption of Food and Nutrition Policies and Litigation: Undermining Government's Role in Public Health. American journal of preventive medicine, 56(1), 47-57.

Pope, C. (2013). Legislating low prices: Cutting costs or care. The Heritage Foundation.(2834), 1-16.

Porter, L. W., Allen, R. W., \& Angle, H. L. (2003). The politics of upward influence in organizations. Organizational influence processes, 2, 431-445.

Powell, W. W., \& DiMaggio, P. J. (Eds.). (2012). The new institutionalism in organizational analysis. University of Chicago press.

Pratt, C. A., Lemon, S. C., Fernandez, I. D., Goetzel, R., Beresford, S. A., French, S. A., ... \& Webber, L. S. (2007). Design characteristics of worksite environmental interventions for obesity prevention. Obesity, 15(9), 2171-2180.

Proper, K., \& van Mechelen, W. (2008). Effectiveness and economic impact of worksite interventions to promote physical activity and healthy diet. Geneva: World Health Organization.

Provan, K. G., \& Kenis, P. (2008). Modes of network governance: Structure, management, and effectiveness. Journal of public administration research and theory, 18(2), 229-252.

Public Health Law Center (PHLC). (2013). Health care Can Lead the Way. William Mitchell College of Law, St. Paul, MN. Retrieved May 52019 from: https://www.publichealthlawcenter.org/sites/default/files/resources/MN.health care.Health care\%20Can\%20Lead\%20the\%20Way.pdf

Puhl, R., Peterson, J. L., \& Luedicke, J. (2013). Fighting obesity or obese persons? Public perceptions of obesity-related health messages. International Journal of Obesity, 37(6), 774.

Ramus, T., \& Vaccaro, A. (2017). Stakeholders matter: How social enterprises address mission drift. Journal of Business Ethics, 143(2), 307-322.

Reed, D. B., \& Chenault, H. J. (2010). Reconstructing the hospital food environment to address the obesity epidemic. Topics in Clinical Nutrition, 25(3), 236-243.

Reilly, J. J., Methven, E., McDowell, Z. C., Hacking, B., Alexander, D., Stewart, L., \& Kelnar, C. J. (2003). Health consequences of obesity. Archives of disease in childhood, 88(9), 748-752.

Roberto, C. A., Swinburn, B., Hawkes, C., Huang, T. T., Costa, S. A., Ashe, M., ... \& Brownell, K. D. (2015). Patchy progress on obesity prevention: emerging examples, entrenched barriers, and new thinking. The Lancet, 385(9985), 24002409.

Robertson, T. S., \& Wind, Y. (1983). Organizational cosmopolitanism and innovativeness. Academy of Management Journal, 26(2), 332-338.

Robles, B., Wood, M., Kimmons, J., \& Kuo, T. (2013). Comparison of nutrition standards and other recommended procurement practices for improving institutional food offerings in Los Angeles County, 2010-2012. Advances in Nutrition, 4(2), 191-202. 
Rodgers, A., Woodward, A., Swinburn, B., \& Dietz, W. H. (2018). Prevalence trends tell us what did not precipitate the US obesity epidemic. The Lancet Public Health, 3(4), e162-e163.

Rodriguez A, Lau AS, Wright B, Regan J, Brookman-Frazee L. Mixed-method analysis of program leader perspectives on the sustainment of multiple child evidencebased practices in a system-driven implementation. Implementation Science. 2018 Dec 1;13(1):44.

Rogers, E.M. (1983). Diffusion of innovations ( $1^{\text {st }}$ ed.). New York: Free Press. Rogers, E.M. (2003). Diffusion of innovations (5th ed.). New York: Free Press. Roman, J. (2015). Solving the Wrong Pockets Problem. Washington, DC: Urban Institute. http://urbnis/2cn1XAX.

Rose, G. (1994). The Strategy of Preventive Medicine. New York: Oxford University Press.

Rutter, H., Bes-Rastrollo, M., De Henauw, S., Lahti-Koski, M., Lehtinen-Jacks, S., Mullerova, D., ... \& Lissner, L. (2017). Balancing upstream and downstream measures to tackle the obesity epidemic: a position statement from the European Association for the Study of Obesity. Obesity facts, 10(1), 61-63.

Sacks, G., Swinburn, B., \& Lawrence, M. (2009). Obesity Policy Action framework and analysis grids for a comprehensive policy approach to reducing obesity. Obesity reviews, 10(1), 76-86.

Sahud, H. B., Binns, H. J., Meadow, W. L., \& Tanz, R. R. (2006). Marketing fast food: impact of fast food restaurants in children's hospitals. Pediatrics, 118(6), 22902297.

Salancik, G. R. (1995). Wanted: A good network theory of organization. Administrative Science Quarterly, 40, 345-349.

Sallis, J. F., \& Glanz, K. (2009). Physical activity and food environments: solutions to the obesity epidemic. The Milbank Quarterly, 87(1), 123-154.

Sarwer, D. B., \& Polonsky, H. M. (2016). The psychosocial burden of obesity. Endocrinology and Metabolism Clinics, 45(3), 677-688.

Schapira, D. V., Kumar, N. B., Lyman, G. H., \& McMillan, S. C. (1990). The value of current nutrition information. Preventive Medicine, 19(1), 45-53.

Schliemann, D., \& Woodside, J. V. (2019). The effectiveness of dietary workplace interventions: a systematic review of systematic reviews. Public health nutrition, 22(5), 942-955.

Schulte PA, Wagner GR, Ostry A, Blanciforti LA, Cutlip RG, Krajnak KM, Luster M, Munson AE, O'Callaghan JP, Parks CG, Simeonova PP. Work, obesity, and occupational safety and health. American journal of public health. 2007;97(3):428-36.

Schulte, P. A., Wagner, G. R., Downes, A., \& Miller, D. B. (2008). A framework for the concurrent consideration of occupational hazards and obesity. Annals of occupational hygiene, 52(7), 555-566.

Schwartz, M. B., Novak, S. A., \& Fiore, S. S. (2009). The impact of removing snacks of low nutritional value from middle schools. Health Education \& Behavior, 36(6), 999-1011. 
Scott, W. (1987). Organizations: Rational, Natural, and Open Systems. Englewood Cliffs, NJ: Prentice Hall.

Scuteri, A., Sanna, S., Chen, W. M., Uda, M., Albai, G., Strait, J., ... \& Dei, M. (2007). Genome-wide association scan shows genetic variants in the FTO gene are associated with obesity-related traits. PLoS genetics, 3(7), e115.

Seidell, J. C. (1998). Societal and personal costs of obesity. Experimental and Clinical Endocrinology \& Diabetes, 106(S02), 7-10.

Seidell, J. C., \& Halberstadt, J. (2015). The global burden of obesity and the challenges of prevention. Annals of Nutrition and Metabolism, 66(Suppl. 2), 7-12.

Selznick, P. (1996). Institutionalism" old" and" new". Administrative science quarterly, 270-277.

Sharf, M., Sela, R., Zentner, G., Shoob, H., Shai, I., \& Stein-Zamir, C. (2012). Figuring out food labels. Young adults' understanding of nutritional information presented on food labels is inadequate. Appetite, 58(2), 531-534.

Shenkin, J. D., \& Jacobson, M. F. (2010). Using the Food Stamp Program and other methods to promote healthy diets for low-income consumers. American Journal of Public Health, 100(9), 1562-1564.

Shill, J., Mavoa, H., Allender, S., Lawrence, M., Sacks, G., Peeters, A., ... \&amp; Swinburn, B. (2012). Government regulation to promote healthy food environments-a view from inside state governments. Obesity reviews, 13(2), 162.

Siervo, M., Wells, J. C., \& Cizza, G. (2009). The contribution of psychosocial stress to the obesity epidemic: an evolutionary approach. Hormone and metabolic research, 41(04), 261-270.

Silverglade, B., \& Heller, I. R. (2010). Food labeling chaos: the case for reform. Center for Science in the Public Interest.

Singh, J. V. (1986). Performance, slack, and risk taking in organizational decision making. Academy of Management Journal, 29(3), 562-585.

Sinha, R., \& Jastreboff, A. M. (2013). Stress as a common risk factor for obesity and addiction. Biological psychiatry, 73(9), 827-835.

Sisnowski, J., Street, J. M., \& Merlin, T. (2017). Improving food environments and tackling obesity: A realist systematic review of the policy success of regulatory interventions targeting population nutrition. PloS one, 12(8), e0182581.

Slappendel, C. (1996). Perspectives on innovation in organizations. Organization Studies, 17(1), 107-129.

Slater, S. J., Powell, L. M., \& Chaloupka, F. J. (2007). Missed opportunities: local health departments as providers of obesity prevention programs for adolescents. American journal of preventive medicine, 33(4), S246-S250.

Smith, A. F. (2017). Food in America: The past, present, and future of food, farming, and the family meal [3 volumes]. ABC-CLIO.

Smith, W. K., Gonin, M., \& Besharov, M. L. (2013). Managing social-business tensions: A review and research agenda for social enterprise. Business Ethics Quarterly, 23(3), 407-442.

Spahlholz, J., Baer, N., König, H. H., Riedel-Heller, S. G., \& Luck-Sikorski, C. (2016). 
Obesity and discrimination-a systematic review and meta-analysis of observational studies. Obesity reviews, 17(1), 43-55.

Snelling, A. M., \& Kennard, T. (2009). The impact of nutrition standards on competitive food offerings and purchasing behaviors of high school students. Journal of School Health, 79(11), 541-546.

Sorensen, G., Stoddard, A., Peterson, K., Cohen, N., Hunt, M.K., Stein, E., Palombo, R. \& Lederman, R., 1999. Increasing fruit and vegetable consumption through worksites and families in the treatwell 5-a-day study. American journal of public health, 89(1), pp.54-60.

Spence, C., Okajima, K., Cheok, A. D., Petit, O., \& Michel, C. (2016). Eating with our eyes: From visual hunger to digital satiation. Brain and cognition, 110, 53-63.

StataCorp. (2019). Stata Statistical Software: Release 16.2. College Station, TX: StataCorp LP.

Stefan, N., Schick, F., \& Haering, H. U. (2017). Causes, characteristics, and consequences of metabolically unhealthy normal weight in humans. Cell metabolism, 26(2), 292-300.

Stender, J. (2018). Geisinger eliminates sugar-sweetened beverages. News Releases, Geisinger. Retrieved 15 September 2019 from: https://www.geisinger.org/aboutgeisinger/news-and-media/news-releases/2018/01/04/16/38/geisinger-eliminatessugar-sweetened-beverages

Stevens, J., Pratt, C., Boyington, J., Nelson, C., Truesdale, K. P., Ward, D. S., ... \& Barkin, S. (2017). Multilevel interventions targeting obesity: research recommendations for vulnerable populations. American journal of preventive medicine, 52(1), 115-124.

STOP Obesity Alliance. (2017). Medicaid fee-for-service treatment of obesity interventions. 50 State and District of Columbia survey. Retrieved 14 April 2019 from:http://stop.publichealth.gwu.edu/sites/stop.publichealth.gwu.edu/files/Cover age\%20PDFs/Medicaid\%20Obesity\%20Coverage\%202017.pdf

Story, M., Kaphingst, K. M., Robinson-O'Brien, R., \& Glanz, K. (2008). Creating healthy food and eating environments: policy and environmental approaches. Annu. Rev. Public Health, 29, 253-272.

Strahan, E. J., Spencer, S. J., \& Zanna, M. P. (2007). Don't take another bite: How sociocultural norms for appearance affect women's eating behavior. Body Image, 4(4), 331-342.

Stunkard, A. J., \& Penick, S. B. (1979). Behavior modification in the treatment of obesity: The problem of maintaining weight loss. Archives of General Psychiatry, 36(7), 801-806.

Swartz, J. J., Braxton, D., \& Viera, A. J. (2011). Calorie menu labeling on quick-service restaurant menus: an updated systematic review of the literature. International Journal of Behavioral Nutrition and Physical Activity, 8(1), 135.

Swinburn, B., Egger, G., \& Raza, F. (1999). Dissecting obesogenic environments: the development and application of a framework for identifying and prioritizing environmental interventions for obesity. Preventive medicine, 29(6), 563-570.

Swinburn, B., Kraak, V., Rutter, H., Vandevijvere, S., Lobstein, T., Sacks, G., ... \& 
Magnusson, R. (2015). Strengthening of accountability systems to create healthy food environments and reduce global obesity. The Lancet, 385(9986), 2534-2545.

Swinburn, B., Sacks, G., Hall, K. D., McPherson, K., Finegood, D. T., Moodie, M. L., \& Gortmaker, S. L. (2011). The global obesity pandemic: shaped by global drivers and local environments. The Lancet, 378(9793), 804-814.

Swinburn, B., Sacks, G., Vandevijvere, S., Kumanyika, S., Lobstein, T., Neal, B., ... \& L'abbé, M. (2013). INFORMAS (International Network for Food and Obesity/non-communicable diseases Research, Monitoring and Action Support): overview and key principles. Obesity reviews, 14, 1-12.

Symonds, C. R., Martins, A. C., \& Hartwell, H. J. (2013). Foodscapes and wellbeing in the workplace: a university setting. Nutrition \& Food Science.

Taber, D. R., Chriqui, J. F., \& Chaloupka, F. J. (2012). Differences in nutrient intake associated with state laws regarding fat, sugar, and caloric content of competitive foods. Archives of pediatrics \& adolescent medicine, 166(5), 452-458.

Taillie, L. S., Reyes, M., Colchero, M. A., Popkin, B., \& Corvalán, C. (2020). An evaluation of Chile's Law of Food Labeling and Advertising on sugar-sweetened beverage purchases from 2015 to 2017: a before-and-after study. PLoS medicine, 17(2), e1003015.

Tesh, S. N., \& Tesh, S. (1988). Hidden arguments: Political ideology and disease prevention policy. Rutgers University Press.

Thompson, J. D. (1967). Organizations in action. New York: McGraw-Hill.

Thorleifsson, G., Walters, G. B., Gudbjartsson, D. F., Steinthorsdottir, V., Sulem, P., Helgadottir, A., ... \& Jonsdottir, T. (2009). Genome-wide association yields new sequence variants at seven loci that associate with measures of obesity. Nature genetics, 4l(1), 18.

Timmer, D., Appleby D., Timmer, V. (2018). United Nations Environment Programme, One Earth (2018). Sustainable Lifestyles: Options and Opportunities in the Workplace. Paris: UN Environment. Retrieved from: https://wedocs.unep.org/bitstream/handle/20.500.11822/27204/SustLifestyles_Wo rk.pdf?sequence $=1$

Tjosvold, D. (1998). Cooperative and competitive goal approach to conflict: Accomplishments and challenges. Applied Psychology, 47(3), 285-313.

Tokar, S. (2019). Workplace sales ban on sugared drink shows positive health effects. News \& Media. University of California San Francisco. Retrieved 2 Feb 2020 from: https://www.ucsf.edu/news/2019/10/415746/workplace-sales-ban-sugareddrink-shows-positive-health-effects

Tong, A., Sainsbury, P., \& Craig, J. (2007). Consolidated criteria for reporting qualitative research (COREQ): a 32-item checklist for interviews and focus groups. International journal for quality in health care, 19(6), 349-357.

Tosti, D. T., \& Jackson, S. F. (2000). Organizational alignment, 2008.

Townshend, T., \& Lake, A. A. (2009). Obesogenic urban form: theory, policy and practice. Health \& place, 15(4), 909-916.

Townshend, T., \& Lake, A. (2017). Obesogenic environments: current evidence of the built and food environments. Perspectives in Public Health, 137(1), 38-44. 
Tremmel, M., Gerdtham, U. G., Nilsson, P., \& Saha, S. (2017). Economic burden of obesity: a systematic literature review. International journal of environmental research and public health, 14(4), 435.

Tushman, M. L., \& Anderson, P. (1986). Technological discontinuities and organizational environments. Administrative science quarterly, 439-465.

US Dept of Health and Human Services. Program announcement number PAR-10-038. Retrieved Oct 12018 from: http://grants.nih.gov/grants/guide/pa-files/PAR-10038.html.

U.S. Department of Health and Human Services and U.S. Department of Agriculture. 2015-2020 Dietary Guidelines for Americans. 8th Edition. December 2015. Retrieved 3 Feb 2020 from: http://health.gov/dietaryguidelines/2015/guidelines/.

USDA Office of Communications. (2014). Fact Sheet: Healthy Hunger Free Kids Act School Meals Implementation. Press Release No. 0098.14. USDA Office of Communications. US Department of Agriculture. Retrieve June 22019 from: https://www.usda.gov/media/press-releases/2014/05/20/fact-sheet-healthyhunger-free-kids-act-school-meals-implementation.

U.S. Department of the Treasury (USDT). (2019) HFFI-FA recipients on Searchable Awards Database. Searched June 62019.

Utterback, J. M. (1974). Innovation in industry and the diffusion of technology. Science, 183(4125), 620-626.

Vandelanotte, C., Sugiyama, T., Gardiner, P., \& Owen, N. (2009). Associations of leisure-time internet and computer use with overweight and obesity, physical activity and sedentary behaviors: cross-sectional study. Journal of medical Internet research, 11(3), e28.

Vandevijvere, S., Chow, C. C., Hall, K. D., Umali, E., \& Swinburn, B. A. (2015). Increased food energy supply as a major driver of the obesity epidemic: a global analysis. Bulletin of the World Health Organization, 93, 446-456.

Van Cauwenberghe, E., Maes, L., Spittaels, H., van Lenthe, F. J., Brug, J., Oppert, J. M., \& De Bourdeaudhuij, I. (2010). Effectiveness of school-based interventions in Europe to promote healthy nutrition in children and adolescents: systematic review of published and 'grey' literature. British journal of nutrition, 103(6), 781797.

Van Duong, T. (2020). A workplace sales ban and motivational intervention can reduce sugar-sweetened beverage intake and improve cardiometabolic health. Evidencebased nursing, 103239.

Van Hulst, A., Barnett, T. A., Déry, V., Côté, G., \& Colin, C. (2013). Health-promoting vending machines: evaluation of a pediatric hospital intervention. Canadian Journal of Dietetic Practice and Research, 74(1), 28-34.

Van Nuys, K., Globe, D., Ng-Mak, D., Cheung, H., Sullivan, J., \& Goldman, D. (2014). The association between employee obesity and employer costs: evidence from a panel of US employers. American journal of health promotion, 28(5), 277-285.

Vander Wekken, S., Sørensen, S., Meldrum, J., \& Naylor, P. J. (2012). Exploring 
industry perspectives on implementation of a provincial policy for food and beverage sales in publicly funded recreation facilities. Health Policy, 104(3), 279287.

Vickers, M. H., Krechowec, S. O., \& Breier, B. H. (2007). Is later obesity programmed in utero?. Current Drug Targets, 8(8), 923-934.

Viera, A. J., Gizlice, Z., Tuttle, L., Olsson, E., Gras-Najjar, J., Hales, D., ... \& Ammerman, A. (2019). Effect of calories-only vs physical activity calorie expenditure labeling on lunch calories purchased in worksite cafeterias. BMC public health, 19(1), 107.

Von Elm, E., Altman, D. G., Egger, M., Pocock, S. J., Gøtzsche, P. C., \& Vandenbroucke, J. P. (2007). The Strengthening the Reporting of Observational Studies in Epidemiology (STROBE) statement: guidelines for reporting observational studies. Annals of internal medicine, 147(8), 573-577.

Wadden, T. A., \& Osei, S. (2002). The treatment of obesity: an overview. Handbook of obesity treatment, $229,248$.

Wallace, R. B., Stratton, K., \& Bonnie, R. J. (Eds.). (2007). Ending the tobacco problem: a blueprint for the nation. National Academies Press.

Wallinga, D. (2010). Agricultural policy and childhood obesity: a food systems and public health commentary. Health Affairs, 29(3), 405-410.

Waltz, T. J., Powell, B. J., Fernández, M. E., Abadie, B., \& Damschroder, L. J. (2019). Choosing implementation strategies to address contextual barriers: diversity in recommendations and future directions. Implementation Science, 14(1), 1-15.

Wang, Y., \& Beydoun, M. A. (2007). The obesity epidemic in the United States-gender, age, socioeconomic, racial/ethnic, and geographic characteristics: a systematic review and meta-regression analysis. Epidemiologic reviews, 29(1), 6-28.

Wang, Y. C., Pamplin, J., Long, M. W., Ward, Z. J., Gortmaker, S. L., \& Andreyeva, T. (2015). Severe obesity in adults cost state Medicaid programs nearly $\$ 8$ billion in 2013. Health Affairs, 34(11), 1923-1931.

Wansink, B., \& Chandon, P. (2006). Meal size, not body size, explains errors in estimating the calorie content of meals. Annals of internal medicine, 145(5), 326332.

Weber, M. (1978). Economy and society: An outline of interpretive sociology. Berkeley: University of California Press.

Weber, M. (2015). Bureaucracy. In Working in America. New York: Routledge.

Weick, K. E. (1976). Educational organizations as loosely coupled systems. Administrative science quarterly, 1-19.

Weick, K. E. (1988). Enacted sensemaking in crisis situations [1]. Journal of management studies, 25(4), 305-317.

Welcome, A. (2019). Definition of Obesity. Obesity Medicine Association. Retrieved June 292019 from: https://obesitymedicine.org/definition-of-obesity/.

Wharton, C. M., Long, M., \& Schwartz, M. B. (2008). Changing nutrition standards in schools: the emerging impact on school revenue. Journal of School Health, 78(5), 245-251.

Whatley Blum, J. E., Davee, A. M., Devore, R. L., Beaudoin, C. M., Jenkins, P. L., 
Kaley, L. A., \& Wigand, D. A. (2007). Implementation of Low-Fat, Low-Sugar, and Portion-Controlled Nutrition Guidelines in Competitive Food Venues of Maine Public High Schools. Journal of School Health, 77(10), 687-693.

White House Conference on Food, Nutrition, and Health: final report. Washington: Government Printing Office; 1970.

Willett, W. (2017). Eat, drink, and be healthy: the Harvard Medical School guide to healthy eating. Simon and Schuster.

Williamson, O. E. (1989). Transaction cost economics. Handbook of industrial organization, 1, 135-182.

Williamson, O. E. (1991). Economic institutions: spontaneous and intentional governance. JL Econ. \& Org., 7, 159.

Witte, K., \& Allen, M. (2000). A meta-analysis of fear appeals: Implications for effective public health campaigns. Health education \& behavior, 27(5), 591-615.

Woo Baidal, J. A., \& Taveras, E. M. (2014). Protecting progress against childhood obesity - the National School Lunch Program. New England Journal of Medicine, 371(20), 1862-1865.

Wojcicki, J. M. (2013). Healthy hospital food initiatives in the United States: time to ban sugar sweetened beverages to reduce childhood obesity. Acta Paediatrica, 102(6), $560-561$.

Wojcicki, J. M., \& Heyman, M. B. (2006). Healthier choices and increased participation in a middle school lunch program: effects of nutrition policy changes in San Francisco. American Journal of Public Health, 96(9), 1542-1547.

Wolfe, I. S. (2007). The perfect labor storm 2.0: Workforce trends that will change the way you do business. Xlibris Corporation.

Wolfenden, L., Barnes, C., Jones, J., Finch, M., Wyse, R. J., Kingsland, M., ... \& Yoong, S. L. (2020). Strategies to improve the implementation of healthy eating, physical activity and obesity prevention policies, practices or programmes within childcare services. Cochrane Database of Systematic Reviews, (2).

Woodward-Lopez, G., Gosliner, W., Samuels, S. E., Craypo, L., Kao, J., \& Crawford, P. B. (2010). Lessons learned from evaluations of California's statewide school nutrition standards. American Journal of Public Health, 100(11), 2137-2145.

Yanovski, S. Z., \& Yanovski, J. A. (2018). Toward precision approaches for the prevention and treatment of obesity. Jama, 319(3), 223-224.

Yilmaz, K. (2013). Comparison of quantitative and qualitative research traditions: Epistemological, theoretical, and methodological differences. European Journal of Education, 48(2), 311-325.

Yan, S., Pappas, A., Vafiadis, D., \& Carson, J. S. (2017). Evaluating Healthy Vending at the American Heart Association National Center: A Pilot Study. Journal of the Academy of Nutrition and Dietetics, 117(9), A55.

Young, L. R., \& Nestle, M. (2002). The contribution of expanding portion sizes to the US obesity epidemic. American journal of public health, 92(2), 246-249.

Zajac, E. J., \& Shortell, S. M. (1989). Changing generic strategies: Likelihood, direction, and performance implications. Strategic Management Journal, 10(5), 413-430.

Zheng, W., Yang, B., \& McLean, G. N. (2010). Linking organizational culture, structure, 
strategy, and organizational effectiveness: Mediating role of knowledge management. Journal of Business research, 63(7), 763-771.

Zick, C. D., Smith, K. R., Fan, J. X., Brown, B. B., Yamada, I., \& Kowaleski-Jones, L. (2009). Running to the store? The relationship between neighborhood environments and the risk of obesity. Social science \& medicine, 69(10), 14931500 .

Zinn, J., \& Flood, A. B. (2009). Commentary: Slack resources in health care organizations-Fat to be trimmed or muscle to be exercised?. Health services research, 44(3), 812.

Zucker, L. G. (1988). Where do institutional patterns come from? Organizations as actors in social systems. Institutional patterns and organizations: Culture and environment, 20(3), 23-49.

Zyngier, S., \& Owen, J. (2013, January). Patterns of Knowledge Management leadership and delegation: supporting an agile organization. In 2013 46th Hawaii International Conference on System Sciences (pp. 3695-3704). IEEE. 


\section{APPENDIX A. Interview Protocol}

Date:

Study ID: Organization

Before initiation of the protocol, researcher will ask respondent if recording can commence. Recording will be saved as "EXEC_[ORG_X].mp3" or “OPER_[ORG_X].mp3”.

I appreciate your willingness to lend your time and insight to my dissertation study focusing on enhancing food environments within health care organizations. Your organization is one of six participating institutions in this study and I am speaking to executive leadership and operational staff from each site in order to gain a rich perspective about the organizational factors that may facilitate or inhibit implementation.

This interview should take about 30 minutes to complete. I will take notes so I can ensure accurate representation of our discussion. Your participation is voluntary and you may end this interview at any time. You don't have to answer any question you don't want to. I will keep our conversation completely confidential and will not make any identifiable details apparent in any final reports in my study. If there is commercially sensitive information reported, please indicate this so that I may exclude those comments from any summaries I develop. I will not attribute any quotes or sentiments to you personally but rather will aggregate insights and perspectives. Given the fact that this conversation is completely confidential, I invite you to be as candid as possible, as the final report will only be as good as the information I collect from participating members!

Please let me know at this time if you have any questions about the consent form or the interview process. Do you have any questions before we begin?

Ensure consent form has been completed. Answer questions. Begin interview.

\section{I will begin by asking you about your organization's actions in addressing the food environment within your [hospital/campus/offices]. (20 min)}

1. What events or actions led [organization] to consider making changes to the internal food environment?

a. Before this, how would you have described the food environment at your organization?

2. How long did it take from acknowledging a need to change the food environment to actual planning and action?

3. Was this change to enhance food environments led by any particular individual? 
a. If so, how did they initiate/prioritize this change?

b. What role did this individual play in your organization?

4. Please describe the specific steps taken by your organization in enhancing the food environment.

a. Has [organization] surveyed opinions of staff/consumers?

b. Has [organization] piloted any changes?

c. Have you instituted new policies and procedures regarding the food and beverage environment?

i. If so, is there any documentation that can be included for time efficiency?

d. Staff education and training?

e. Employee and/or consumer education?

f. Procurement practices?

5. Have there been any barriers in enhancing the food environment?
a. Disgruntled staff/employee/consumers?
b. Stakeholder resistance?
c. Fears?
d. Other barriers?

6. What have been facilitators that have aided this process?

a. Available resources for transition?

b. Champions?

c. Buy-in among staff/consumers?

d. Other facilitators?

7. What kinds of monitoring efforts are in place to evaluate the food environment?

a. Before the [initiative/program]?

b. Are any data available yet to evaluate effects of the standards?

c. Do you plan measuring sales data to inform procurement practices?

8. Please describe how visible your organization's efforts were in marketing the improvements to the food environment.

a. Have these changes been implemented relatively under the radar and/or over time or as a part of a formalized initiative or program?

b. How has your organization marketed the changes to consumers, staff, public?

c. How has the reception been by:

i. The public/media

ii. Staff

iii. Employees

iv. Other groups (e.g. medical staff) 
The next set of questions are about your organization's characteristics. (10 minutes)

9. How are the goals of your organization's stakeholders aligned? How do they diverge?

10. How are conflicts resolved among parties?

11. What is the nature of communication among stakeholders?

a. Would you consider communication channels to be relaxed or strained?

12. Say your organization wanted to learn more about a new technology or practice. What resources are available?

a. Network resources? Coalitions? Partnerships?

b. Organizational resources? (e.g., dedicated office or personnel?)

c. Are there resources available to hire outside consultation?

13. Which channels of communication does your organization rely on to get credible information? To hear about new practice patterns or interventions?

14. Describe the degree of interorganizational cooperation needed (from partners, government, competitors) for your organization to achieve strategic goals?

a. Is there a history of conflict or cooperation with local entities or state government?

b. If applicable: Do outpatient clinics or sites operate with a relative degree of freedom when making local decisions?

15. How strong would you say is your organization's impetus to "model health" for others - would you say very strong, pretty strong, or not too strong? What leads you to say [how the interviewee responded]?

a. How would you rate your organization's internal climate in addressing pressing public health problems?

b. Are there any practice patterns that you feel are discordant with "modeling health"?

c. What short- and long-term external pressures do you feel your organization needs to address in the next 5-10-15 years?

We've reached the end of the interview. Thank you for your candor and insight! Is it alright if I contact you again for any clarifications? 


\section{APPENDIX B. Recruitment Email Invitation to Prospective Interview Participants}

[Date]

Subject Line: Interview Request for Doctoral Research Study

Dear [Name of Potential Interview Subject],

My name is Liz Walker and I am a doctoral candidate in the Health Systems and Policy $\mathrm{PhD}$ program at the OHSU-PSU School of Public Health. I am conducting a research study as part of my dissertation work under the supervision of Dr. Julia Goodman, Assistant Professor of Public Health (jmg@pdx.edu).

You are invited to participate in an interview as part of a research study that seeks to explore factors related to improving the food environments of health care organizations.

My dissertation work explores the organizational challenges of implementing HFEPs (FYI: standards regarding procurement practices, not inpatient dietary standards). My aim is to involve a diverse array of health care organizations in order to flesh out barriers to implementation while identifying how organizational factors can influence the kinds of challenges faced-- e.g., does the size of an organization pose as a challenge, the degree to which mission/vision of an organization facilitates healthy procurement practices, the importance of slack resources, etc.

As food environments are increasingly recognized as drivers of the obesity epidemic, I am interested in exploring how hospitals and health systems are implementing changes to cafeterias and onsite food venues. Being able to identify implementation challenges and link them to organizational factors can be helpful for other organizations when deciding how to go about making healthy food and beverage environments.

Who do I want to interview? Executive leadership and operational managers of organizations that have taken steps to improve food and beverage environments.

What would we talk about? The following topics:

- Your organization's process of recognizing the importance of food environments

- Your organization's organizational characteristics (e.g. governance style, channels of communication, long-term goals, etc.)

- What planning and steps have been taken to improve the food and beverage environment

- What challenges arose during the implementation process

- Your insight on key stakeholders that are important to this process 
- How you see your organization progress in creating healthy contexts and modeling healthy behaviors in the future

How long would this interview take? The interview would take approximately 30 minutes and I would be happy to meet you in-person at [Organization] for your convenience. If meeting in-person is ultimately not possible, we could arrange to speak by phone or Zoom.

This sounds great! What's the next step? If you are interested in learning more about the study or being interviewed, please respond to this email (kvachel@pdx.edu) or call me at 503-998-6901. If you are not interested, please let me know so that I will not continue to contact you.

I have attached the consent form that provides more detail of my research study as well as your rights as a research subject in this work. This study is under the oversight of my doctoral committee as well as the Human Research Protection Program of Portland State University. If you have any concerns or questions, please e-mail psuirb@pdx.edu or call 503-725-4288.

Your insight and perspective will be valuable information for this study. If you agree to participate, please let me know and we will find a convenient time in January for the interview. I appreciate your time and consideration!

Best regards, Liz Walker

kvachel@pdx.edu 


\section{APPENDIX C. Interview Consent Form}

\section{ALIGNING FOOD ENVIRONMENTS WITH INSTITUTIONAL VALUES: A MIXED METHODS STUDY OF OREGON HEALTH CARE ORGANIZATIONS \\ Interview Consent Form}

\section{INVESTIGATOR}

Elizaveta Walker, MPH

Telephone: 503-998-6901

Email: kvachel@pdx.edu

\section{OVERSIGHT CONTACT INFORMATION}

Julia Goodman, PhD, MPH

Assistant Professor of Public Health

Chair of Doctoral Dissertation Committee

Telephone: 503-725-2225

Email: jmg@pdx.edu

-and-

Human Research Protection Program, Institutional Review Board, Portland State University

Address: 620 Market Center Building, 1600 SW $4^{\text {th }}$ Ave, Portland, OR 97201

Telephone: $503-725-4288$ or 1-877-480-4400

Email: psuirb@pdx.edu

\section{PURPOSE}

You are invited to participate in an interview as part of a research study that seeks to explore factors related to improving food environments of health care organizations. Elizaveta Walker is conducting interviews with operational managers and executive leadership among six health care organizations in Oregon. This research study is under the oversight of Dr. Julia Goodman, Assistant Professor in the OHSU-PSU School of Public Health.

As food environments are increasingly recognized as major drivers of the obesity epidemic, Elizaveta Walker is interested in exploring how hospitals and health systems are looking inward and implementing changes to cafeterias and onsite food venues. Being able to identify implementation challenges and link them to organizational factors can be helpful for others when deciding how to go about making healthy food and beverage environments. 


\section{STUDY PROCEDURE}

Your participation in this study will consist of one (1) in-person interview lasting approximately 30 minutes with Elizaveta Walker. If an in-person interview is not possible, a telephone or Zoom session will be arranged. With your permission, the interview will be audio-recorded. None of your identifying information will be used. The recording will be used for the exclusive purpose of helping Elizaveta Walker preserve your sentiments accurately. You may decline participation in the future or end participation at any point in the study.

\section{POSSIBLE RISKS \& BENEFITS}

During the interview, some questions asked may be sensitive in nature and/or make you uncomfortable. You can refuse to answer any question(s) asked during the interview and this will not affect your participation.

Any information obtained through participation in this study will be confidential and exclusively used by the researcher and no one else. Each participating organization will be kept confidential. Given that six Oregon health care organizations have been included in this study, it may be somewhat difficult to completely blind the organization, but I will de-identify individual respondents. The master spreadsheet that links organizations with their study ID will be kept in a password-protected file on the researcher's computer. Audio recordings of the interviews will be permanently deleted once transcribed. Interview transcripts will be destroyed three years after the interview is completed.

All study records generated from this interview will be kept in the custody of the researcher. As part of the oversight done by Portland State University Institutional Review Board (IRB), the oversight committee may request access to the interview transcripts and be able to look at the study records. Your specific responses will be kept confidential.

\section{GENERAL INFORMATION}

Your participation is voluntary and you may end your participation in this study at any time. Your responses and decision to participate will not affect your relationships with Portland State University or Oregon Health \& Science University.

You do not give up any legal rights by signing this consent form or taking part in the study.

If you have any questions or concerns regarding your participation in this study, you can contact the Human Research Protection Program of Portland State University by email: psuirb@pdx.edu or by telephone: 503-725-4288 or 1-877-480-4400. If you have any 
other concerns about the study, you may contact Dr. Julia Goodman by telephone: 503725-2225 or by email: jmg@pdx.edu, or me directly by telephone: 503-998-6901 or by email:kvachel@pdx.edu.

\section{SIGNATURES}

A copy of this consent form will be provided for your reference.

Name of Participant (Please Print)

Signature of Participant

Date

This research study has been explained to the participant and all questions and concerns have been addressed. The participant freely consents to participant and has no further questions at this time.

Elizaveta Walker

Name of Investigator

Signature of Investigator

Date 


\section{APPENDIX D. Consolidated Criteria for Reporting Qualitative Studies Checklist}

\begin{tabular}{|c|c|c|c|}
\hline Domain & $\begin{array}{l}\text { Item } \\
\text { No. }\end{array}$ & Guided Question & $\begin{array}{l}\text { Included? } \\
\text { (Yes/NA) }\end{array}$ \\
\hline \multicolumn{4}{|c|}{ Research team and reflexivity } \\
\hline Interviewer & 1 & Which author conducted the interview? & Yes \\
\hline Credentials & 2 & What were the researcher's credentials? & Yes \\
\hline Occupation & 3 & What was their occupation at the time of study? & Yes \\
\hline Gender & 4 & Was the researcher male or female? & NA \\
\hline Experience & 5 & $\begin{array}{l}\text { What experience or training did the researcher } \\
\text { have? }\end{array}$ & NA \\
\hline \multicolumn{4}{|c|}{ Relationship w participants } \\
\hline $\begin{array}{l}\text { Relationship } \\
\text { established }\end{array}$ & 6 & Was a relationship established prior to study? & Yes \\
\hline $\begin{array}{l}\text { Participant } \\
\text { knowledge }\end{array}$ & 7 & $\begin{array}{l}\text { What did the participants know about the } \\
\text { researcher? }\end{array}$ & NA \\
\hline $\begin{array}{l}\text { Interviewer } \\
\text { characteristics }\end{array}$ & 8 & $\begin{array}{l}\text { What characteristics were reported about the } \\
\text { interviewer/facilitator? }\end{array}$ & NA \\
\hline \multicolumn{4}{|c|}{ Theoretical framework } \\
\hline $\begin{array}{l}\text { Methodological } \\
\text { orientation \& } \\
\text { theory }\end{array}$ & 9 & $\begin{array}{l}\text { What methodological orientation was stated to } \\
\text { underpin the study? }\end{array}$ & Yes \\
\hline \multicolumn{4}{|c|}{ Participant selection } \\
\hline Sampling & 10 & How were participants selected? & Yes \\
\hline $\begin{array}{l}\text { Method of } \\
\text { approach }\end{array}$ & 11 & $\begin{array}{l}\text { How were participants approached? e.g. face- } \\
\text { to-face, telephone, mail, email }\end{array}$ & Yes \\
\hline Sample size & 12 & How many participants were in the study? & Yes \\
\hline Non-participation & 13 & $\begin{array}{l}\text { How many people refused to participate or } \\
\text { dropped out? Reasons? }\end{array}$ & Yes \\
\hline \multicolumn{4}{|c|}{$C_{T P}$} \\
\hline $\begin{array}{l}\text { Setting of data } \\
\text { collection }\end{array}$ & 14 & $\begin{array}{l}\text { Where was the data collected? E.g. home, } \\
\text { clinic }\end{array}$ & Yes \\
\hline $\begin{array}{l}\text { Presence of non- } \\
\text { participants }\end{array}$ & 15 & $\begin{array}{l}\text { Was anyone else present besides the } \\
\text { participants and researchers? }\end{array}$ & Yes \\
\hline $\begin{array}{l}\text { Description of } \\
\text { sample }\end{array}$ & 16 & $\begin{array}{l}\text { What are the important characteristics of the } \\
\text { sample? Eg demographic data, date }\end{array}$ & Yes \\
\hline \multicolumn{4}{|l|}{ Data collection } \\
\hline Interview guide & 17 & $\begin{array}{l}\text { Were questions, prompts, guides provided by } \\
\text { the authors? Was it pilot tested? }\end{array}$ & Yes \\
\hline Repeat interviews & 18 & $\begin{array}{l}\text { Were repeat interviews carried out? If yes, how } \\
\text { many? }\end{array}$ & Yes \\
\hline $\begin{array}{l}\text { Audio/visual } \\
\text { recording }\end{array}$ & 19 & $\begin{array}{l}\text { Did the research use audio or visual recording } \\
\text { to collect the data? }\end{array}$ & Yes \\
\hline Field notes & 20 & $\begin{array}{l}\text { Were field notes made during and/or after the } \\
\text { interview? }\end{array}$ & Yes \\
\hline Duration & 21 & What was the duration of the interviews? & Yes \\
\hline Data saturation & 22 & Was data saturation discussed? & NA \\
\hline $\begin{array}{l}\text { Transcripts } \\
\text { returned }\end{array}$ & 23 & $\begin{array}{l}\text { Were transcripts returned to participants for } \\
\text { comment and/or correction? }\end{array}$ & Yes \\
\hline
\end{tabular}




\begin{tabular}{|l|l|l|l|}
\hline Analysis and findings & How many data coders coded the data? & Yes \\
\hline $\begin{array}{l}\text { Number of data } \\
\text { coders }\end{array}$ & 24 & $\begin{array}{l}\text { Did authors provide a description of the coding } \\
\text { tree? }\end{array}$ & NA \\
\hline $\begin{array}{l}\text { Description of } \\
\text { coding tree }\end{array}$ & 26 & $\begin{array}{l}\text { Were themes identified in advance or derived } \\
\text { from the data? }\end{array}$ & Yes \\
\hline $\begin{array}{l}\text { Derivation of } \\
\text { themes }\end{array}$ & 27 & $\begin{array}{l}\text { What software, if applicable, was used to } \\
\text { manage the data? }\end{array}$ & Yes \\
\hline Software & 28 & $\begin{array}{l}\text { Did participants provide feedback on the } \\
\text { findings? }\end{array}$ & Yes \\
\hline $\begin{array}{l}\text { Participant } \\
\text { checking }\end{array}$ & 29 & $\begin{array}{l}\text { Were participant quotations presented to } \\
\text { illustrate the themes / findings? Was each } \\
\text { quotation identified? }\end{array}$ & Yes \\
\hline Reporting & 30 & $\begin{array}{l}\text { Was there consistency between the data } \\
\text { presented and the findings? }\end{array}$ & Yes \\
\hline $\begin{array}{l}\text { Quotations } \\
\text { presented }\end{array}$ & 31 & $\begin{array}{l}\text { Were major themes clearly presented in the } \\
\text { findings? }\end{array}$ & Yes \\
\hline $\begin{array}{l}\text { Data and findings } \\
\text { consistent }\end{array}$ & 32 & $\begin{array}{l}\text { Is there a description of diverse cases or } \\
\text { discussion of minor themes? }\end{array}$ & Yes \\
\hline $\begin{array}{l}\text { Clarity of major } \\
\text { themes }\end{array}$ & $\begin{array}{l}\text { Clarity of minor } \\
\text { themes }\end{array}$ & & \\
\hline
\end{tabular}




\section{APPENDIX E. Organizational Context Scores for All Variables}

Full scores of all variables, by organization

\begin{tabular}{|c|c|c|c|c|c|c|}
\hline Identifier & 1 & 2 & 3 & 4 & 5 & 6 \\
\hline Structure & $+/-$ & + & $+/-$ & $+/-$ & $+/-$ & $+/-$ \\
\hline Governance & - & + & - & $+/-$ & + & $+/-$ \\
\hline Size & $+/-$ & + & $+/-$ & + & $+/-$ & - \\
\hline $\begin{array}{l}\text { Interorganizational } \\
\text { Coupling }\end{array}$ & $+/-$ & $+/-$ & - & $+/-$ & $+/-$ & - \\
\hline Receptivity & + & + & - & $+/-$ & + & - \\
\hline Norms and culture & + & + & - & $+/-$ & + & $+/-$ \\
\hline Tension for change & + & + & - & $+/-$ & $+/-$ & - \\
\hline Resource Allocation & - & + & - & + & + & $+/-$ \\
\hline Slack resources & - & + & - & + & + & $+/-$ \\
\hline Social network location & $+/-$ & $+/-$ & - & + & $+/-$ & - \\
\hline Information sharing & - & + & - & + & + & + \\
\hline Leadership & - & + & - & + & $+/-$ & + \\
\hline Champion & - & + & $+/-$ & + & $+/-$ & + \\
\hline Stakeholders & - & + & - & $+/-$ & $+/-$ & $+/-$ \\
\hline Vision & $+/-$ & + & - & $+/-$ & + & + \\
\hline
\end{tabular}

